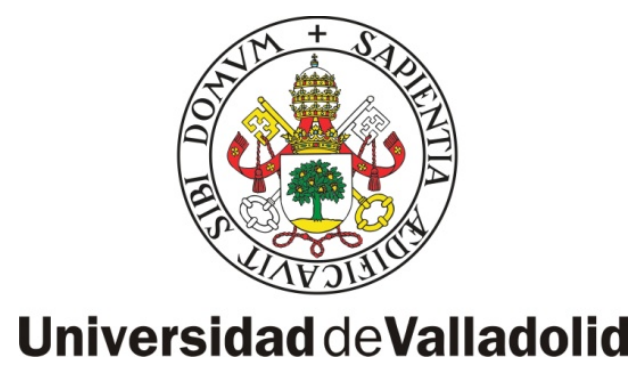

Escuela de Ingenierías Industriales

Departamento de Organización de Empresas y C.I.M

TESIS DOCTORAL

\title{
Emergencia de Normas Económicas en Sociedades Artificiales de Agentes. Contribuciones al Juego de la Demanda de Nash.
}

\author{
Presentada por D. David J. Poza García \\ para optar al grado de doctor por la \\ Universidad de Valladolid
}

Dirigida por Dr. Adolfo López Paredes y Dr. José Manuel Galán Ordax 



\section{AGRADECIMIENTOS}

Quiero agradecer a mis seres queridos por ser, en todo momento, los principales pilares de mis éxitos.

A mis directores de tesis, Dr. Adolfo López-Paredes y Dr. José Manuel Galán Ordax, por su incondicional apoyo e importantes aportes durante estos años de trabajo.

A Nacho por las numerosas ocasiones en las que ha compartido sus conocimientos conmigo. Sin duda, esta tesis no sería lo mismo sin su ayuda.

Un especial agradecimiento a Félix Villafáñez, cuya disposición para ayudar a sus compañeros no conoce límites y con quien he tenido la suerte de estar acompañado desde que inicié mi vida profesional.

Finalmente quiero agradecer a todos mis compañeros del grupo INSISOC que de alguna manera u otra han hecho de esta tesis una realidad. 

Esta tesis doctoral ha sido realizada gracias a la obtención de la beca de Formación de Personal Investigador (2010BES-2009-016416) en el proyecto SiCoSSys (TIN2008-06464C03-01). 



\section{ÍNDICE DE CONTENIDOS}

BLOQUE I................................................................ 13

1. INTRODUCCIÓN .......................................................... 15

1.1. ASPECTOS GENERALES Y MOTIVACIÓN ………………….................15

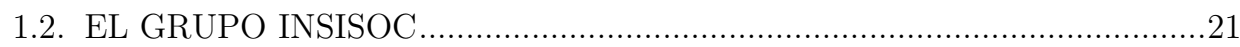

1.3. UBICACIÓN DE LA INVESTIGACIÓN DENTRO DE LAS CORRIENTES

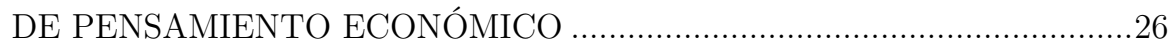

2. MARCO METODOLÓGICO ................................................ 33

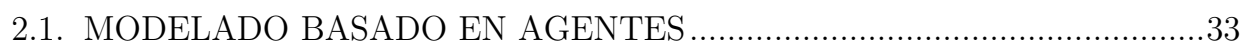

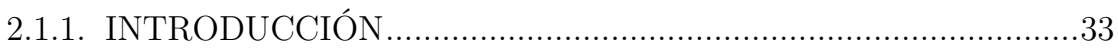

2.1.2. DEFINICIÓN DE AGENTE Y MODELADO ...............................34

2.1.3. CARACTERÍSTICAS DEL MODELADO BASADO EN AGENTES.

2.1.4. MODELADO BASADO EN AGENTES Y COMPORTAMIENTO EMERGENTE

2.1.5. BUENAS PRÁCTICAS EN LA CONSTRUCCIÓN DE MODELOS BASADOS EN AGENTES.

2.1.6. LA DIFICULTAD DE MODELAR EL COMPORTAMIENTO HUMANO

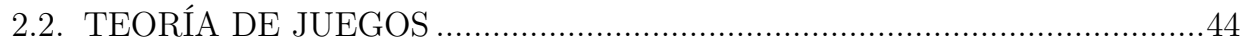

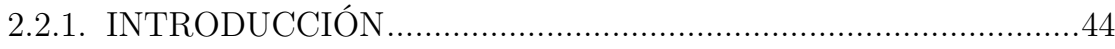

2.2.2. LA MATRIZ DE PAGOS .......................................................... 45

2.2.3. EQUILIBRIO DE NASH Y ÓPTIMOS EN SENTIDO SOCIAL Y DE PARETO. CONCEPTOS RELACIONADOS.

2.2.4. ANTECEDENTES DEL EQUILIBRIO DE NASH Y TRABAJOS

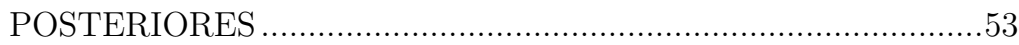

2.2.5. TEORÍA DE JUEGOS Y ECONOMÍA …...................................54

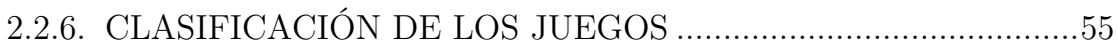

2.2.7. RAMAS DE LA TEORÍA DE JUEGOS ……………...................56

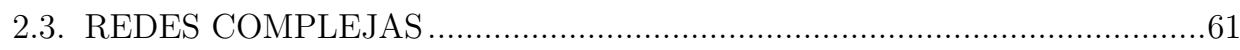

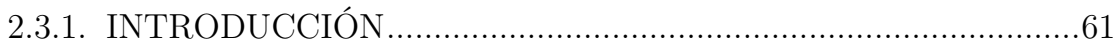

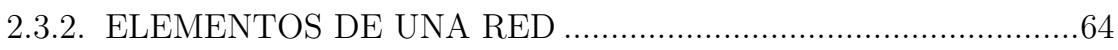


2.3.3. PROPIEDADES BÁSICAS DE UNA RED .65

2.3.4. CONCEPTOS RELACIONADOS ...............................................

2.3.5. MODELOS TEÓRICOS DE REDES ...........................................78

3. EL JUEGO DE LA DEMANDA DE NASH ......................... 87

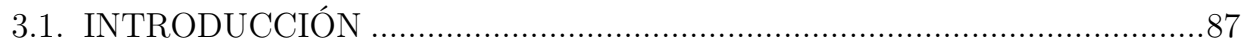

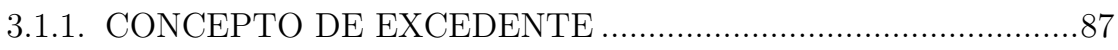

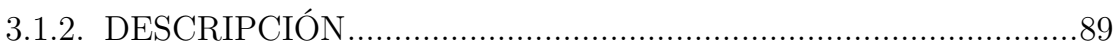

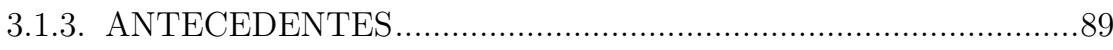

3.1.4. MODIFICACIONES EN EL JUEGO DE LA DEMANDA DE

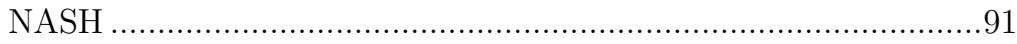

3.2. EL MODELO DE CLASES DE AXTELL, EPSTEIN Y YOUNG ……........95

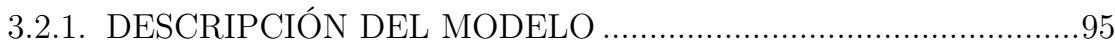

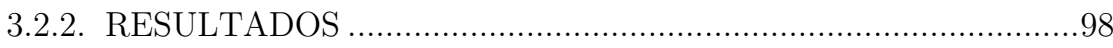

3.3. ANÁLISIS DE SENSIBILIDAD E INFLUENCIA DE CONDICIONES INICIALES. NUEVA REGLA DE DECISIÓN ………………...................... 106

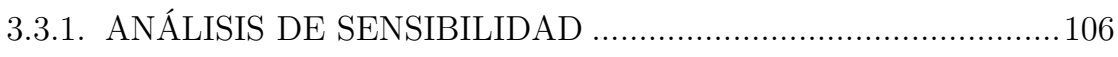

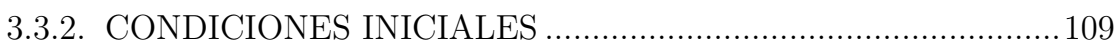

3.3.3. NUEVA REGLA DE DECISIÓN ……………….......................110

3.4. AGENTES SITUADOS EN UN GRID REGULAR ..................................114

3.4.1. DISTRIBUCIÓN DE LA TAG EN DOS ZONAS ......................116

3.4.2. DISTRIBUCIÓN DE LA TAG EN CUATRO ZONAS................119

3.4.3. DISTRIBUCIÓN DE LA TAG DE MANERA ALEATORIA ....121

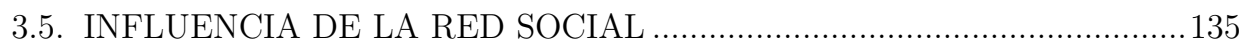

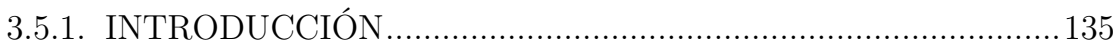

3.5.2. PROPIEDADES DE LAS REDES SOCIALES Y MODELO

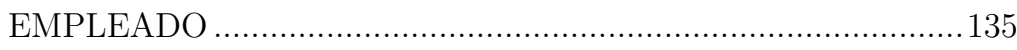

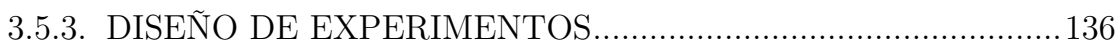

3.5.4. INFLUENCIA DEL CLUSTERING EN LA EMERGENCIA DE LA NORMA PARITARIA 137

3.5.5. INFLUENCIA DEL CLUSTERING EN LA DIFUSIÓN DE LA NORMA PARITARIA 
New Insights on the Emergence of Classes Model .......................173

Mesoscopic Effects in an Agent-Based Bargaining Model in Regular Lattices .193

Evolution of the Equity Norm in Small World Networks .207

Anexo: Evaluación de las revistas en las que han sido publicados los artículos presentados en esta tesis doctoral.............................231 Discrete Dynamics in Nature and Society .............................233

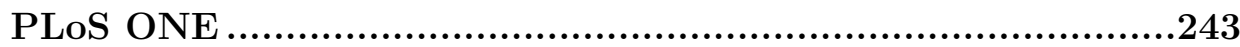





\section{Presentación}

El trabajo realizado en esta tesis doctoral se presenta estructurado en dos bloques:

El primero de ellos se desarrolla en tres capítulos en los que se comienza por presentar una Introducción que recoge los antecedentes de esta tesis doctoral, su relación con otras líneas de investigación en INSISOC y dentro del área de conocimiento de Organización de Empresas. El segundo capítulo está dedicado a presentar el marco metodológico, en concreto el Modelado Basado en Agentes y la Teoría de Juegos y las Redes Complejas. El tercer capítulo presenta el problema de estudio en el que nos hemos centrado en la investigación y sintetiza los principales resultados a los que ha dado lugar nuestro trabajo. Concluimos este primer bloque con la identificación de las conclusiones más relevantes de esta tesis doctoral.

El segundo bloque recoge las tres publicaciones principales, en revistas indizadas del primer cuartil de los Journal Citation Reports (JCR) de ISI Thomson:

- New Insights on the Emergence of Classes Model, publicado en 2011 en la revista Discrete Dynamics in Nature and Society. (Poza et al., 2011).

- Mesoscopic Effects in an Agent-based Bargaining Model in Regular Lattices, publicado en 2011 en la revista PLoS ONE. (Poza et al., 2011).

- Evolution of Equity Norms in Small-World Networks, publicado en 2012 en la revista Discrete Dynamics in Nature and Society. (Santos et al., 2012). 

BLOQUE I 



\section{INTRODUCCIÓN}

\subsection{ASPECTOS GENERALES Y MOTIVACIÓN}

La línea central de esta tesis es el problema del intercambio. Entendemos por intercambio el problema que surge a la hora de repartir un excedente entre dos individuos. El contexto de nuestro estudio son aquellas situaciones de negociación que tienen lugar de manera repetitiva, de tal modo que, al cabo de cierto tiempo, acaba emergiendo una norma que gobierna el intercambio, sin la necesidad de la existencia de una autoridad central que se encargue de su cumplimiento.

En concreto, centraremos nuestro análisis en aquellas situaciones en las que el intercambio es eficiente (es decir, aquellos casos en los que la totalidad del excedente es repartida) y equitativo (el excedente es repartido a partes iguales entre los agentes que reciben el excedente).

Veamos un ejemplo de reparto de un excedente. Sean dos individuos. Uno de ellos desea vender un bien por el que no está dispuesto a aceptar una cantidad inferior a 1.000€. El segundo individuo está interesado en adquirir dicho bien, pero no está dispuesto a gastar más de $1.100 €$ en el mismo. Para que se produzca el intercambio, el precio de compraventa deberá estar comprendido entre $1.000 €$ y $1.100 €$. Es decir, existe un excedente de $100 €$ que los dos individuos deberán negociar. El excedente del comprador se define como la diferencia entre el precio máximo que ésta está dispuesto a pagar por el bien y el precio de compra-venta; el excedente del vendedor es la diferencia entre el precio de venta y el precio mínimo al que está dispuesto a vender. Así, por ejemplo, si finalmente el precio de compra-venta es de 1.070€, el excedente del comprador será de $30 €$ y el excedente del vendedor será de $70 €$. Es decir, como resultado de la negociación, al vendedor le ha correspondido un $70 \%$ y al comprador un $30 \%$ del excedente. En este caso, el reparto del excedente ha sido eficiente (el cien por cien del excedente ha sido repartido entre los dos agentes) pero no ha sido equitativo (el excedente no se ha repartido a partes iguales). Como veremos más adelante, existen otras situaciones en las 
que el intercambio, además de ser eficiente, también es equitativo y también otras situaciones en las que el intercambio ni siquiera es eficiente.

En el ejemplo anterior tan sólo contábamos con dos individuos y la transacción tenía lugar una única vez. Sin embargo, nuestro interés en esta investigación se centra en aquellos escenarios en los que la negociación tiene lugar de manera repetitiva y con un número elevado de individuos. En este tipo de escenarios, con el paso del tiempo, los individuos van aprendiendo a partir de su experiencia en negociaciones pasadas y adaptan su estrategia para tratar de maximizar su beneficio en futuras negociaciones. Como resultado de este aprendizaje, puede ocurrir que emerjan ciertas normas en el sistema, haciendo que ciertos grupos de individuos acaben actuando de una manera bien diferenciada.

Algunos ejemplos de estas normas emergentes son el reparto del dinero entre el bufete de abogados y su cliente tras ganar un juicio en EEUU (reparto $1 / 3$ - 2/3) o el reparto del beneficio de una tierra de cultivo entre el terrateniente y el arrendatario (generalmente 1/2 - 1/2) (Young, 1998). A pesar de que esta manera de repartir el excedente no se encuentra regulada por una autoridad central, lo que ha ocurrido con estas fracciones de reparto es que se han acabado convirtiendo en una norma con el paso del tiempo. Las normas actúan como mecanismos reguladores del comportamiento de los individuos sin que sea necesaria la existencia de una autoridad central que imponga su cumplimiento: es la propia sociedad la que se encarga de hacerlas cumplir. Este mecanismo regulador ha sido estudiado en profundidad en las ciencias sociales (Coleman, 1990; Kandori, 1992).

Precisamente, uno de los objetivos de esta tesis es analizar por qué surgen, se difunden y persisten en la sociedad algunas normas que acaban institucionalizándose y, por tanto, afectando a la manera en la que se produce el intercambio económico.

Concretamente, en el campo de la Economía, se ha llevado a cabo un esfuerzo importante en el estudio de la emergencia y difusión de normas que regulan la distribución de la propiedad dentro de una comunidad. Es más, existe evidencia de que algunas interacciones se regulan más bien a través de hábitos, relaciones repetitivas entre individuos, presión social, confianza o reputación que a través de contratos (Kandori, 1992). Por un lado, existen normas paritarias que conducen a una "división justa" de los bienes, y por otro existen normas discriminatorias que asignan diferentes 
proporciones del recurso a repartir en función de alguna característica del individuo o bien por la pertenencia de éste a algún grupo caracterizado por ciertos atributos (sexo, raza, edad, etc.).

Normalmente, el resultado del reparto (e incluso la posibilidad de que el reparto llegue a realizarse) no depende solamente de las decisiones individuales de cada individuo, sino de la combinación de ambas decisiones. Es decir, nos encontramos ante una situación de decisiones interdependientes, adentrándonos así en el ámbito de la Teoría de Juegos, uno de los marcos metodológicos que hemos empleado en esta tesis. Concretamente, la

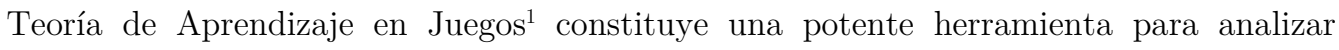
formalmente este tipo de normas (Izquierdo, Izquierdo \& Vega-Redondo, 2012; Fudenberg \& Levine, 1998; Young, 2004; Vega-Redondo, 2003). Mediante esta aproximación, las interacciones sociales pueden modelarse como juegos en los que diversos jugadores utilizan $\mathrm{su}$ memoria sobre experiencias pasadas para formar expectativas sobre el comportamiento de los otros jugadores, basando en ellas la selección de su estrategia.

Nuestra línea de investigación sigue la propuesta de Vernon Smith (1989) en cuanto al diseño de experimentos (institución, entorno y agentes). No obstante, nos distanciamos de su metodología en tanto que nosotros sustituiremos la experimentación con humanos por simulación con agentes software (López Paredes, 2001; López-Paredes, HernándezIglesias \& Gutiérrez Pajares, 2002). La cuestión es que estos agentes software deben estar "programados" para interactuar de alguna manera con su entorno y con otros agentes según unas normas de comportamiento. Para abordar este problema, utilizaremos como herramienta el Modelado Basado en Agentes. Esta técnica nos permite definir un (micro) comportamiento para cada uno de los agentes del sistema, para después poder observar el (macro) comportamiento que surge a partir de la interacción de los individuos del sistema.

En el caso concreto de normas de distribución de propiedad, las interacciones se modelan a menudo como juegos de demanda de Nash (1950). En este juego, dos jugadores deben repartirse cierta cantidad de un bien. Los jugadores reciben la cantidad del bien que solicitaron siempre que la suma de ambas cantidades sea, como máximo, la totalidad del 
bien a repartir; en caso contrario ninguno de ellos recibe nada. Basándose en este juego y en su posterior versión evolutiva (Young, 1993), Axtell, Epstein y Young (2000) diseñaron un modelo basado en agentes (en adelante, modelo de AEY) para estudiar la dinámica transitoria y asintótica del juego de la demanda de Nash en una población finita. En su modelo, se asumía que los jugadores podían escoger entre tres posibles demandas: low, medium y high (30\%, 50\% y 70\% del bien a repartir, respectivamente). Los agentes elegían la estrategia que maximizaba su beneficio en función de sus expectativas sobre la estrategia del oponente. Este modelo muestra que varios regímenes persistentes diferentes a la norma paritaria pueden emerger y perpetuar bajo varias reglas de aprendizaje y combinaciones de parámetros.

Como paso previo a la extensión del modelo de AEY, procedimos a la replicación del mismo (Poza et al., 2011). Partimos del hecho de que el resultado de una simulación sólo puede ser fiable si ésta puede ser reproducida por otra persona partiendo de cero, ya que, de lo contrario, podría ocurrir que los resultados publicados estuvieran equivocados debido a errores de programación, a una representación incorrecta de lo que realmente se está simulando, etc. (Axelrod, 1997). La replicación de modelos de forma independiente permite descubrir debilidades en el modelo original que de otra manera no habrían salido a la luz (Edmonds \& Hales, 2003). Además, durante la replicación del modelo, se prestó una atención especial a las hipótesis que deben formularse durante el diseño del modelo, buscando posibles artefactos (Galán et al., 2009; Kubera, Mathieu \& Picault, 2009).

Los resultados que nos proporcionó nuestra réplica del modelo de AEY estaban en consonancia con los trabajos de López-Paredes, Hernández y Pajares (2004) y de Dessalles (2007), quienes habían llevado a cabo la replicación de este modelo con anterioridad. Una vez replicado el modelo, se llevó a cabo un análisis de sensibilidad en el que se estudió cómo afectan algunos elementos del sistema a los resultados de la simulación. Concretamente, se analiza la influencia de la matriz de pagos y de la configuración de la memoria en la que los agentes almacenan información para la toma de decisiones. Posteriormente se dotó a los agentes de una nueva regla de decisión que requería menos habilidades cognoscitivas que la regla de decisión original, concluyendo que la introducción de esta nueva regla de decisión, si bien no producía variación en los regímenes alcanzados por el sistema, la dinámica del mismo sí se veía afectada.

Posteriormente llevamos a cabo una extensión del modelo original con el fin de analizar el efecto de la estructura de interacción entre los agentes en los resultados (Poza et al., 
2011). En particular, analizamos el modelo de AEY en grids regulares con una población finita de agentes dotados de una tag (o etiqueta que los identifica dentro de un grupo), descubriendo que las propiedades mesoscópicas de la red de interacción tienen un impacto significativo en la difusión de las estrategias. En concreto, observamos que, debido a la distribución aleatoria de la tag en la red, pueden surgir propiedades topológicas (clústers aislados, estructuras de comunidad, etc.) que explican la difusión de las estrategias de los agentes en el grid y la emergencia de nuevos regímenes estables que no aparecían en el modelo original de AEY.

En cualquier caso, las redes de interacción reales normalmente difieren de la topología regular de un grid bidimensional (Newman, 2003). Para profundizar en los efectos de la estructura social en la difusión de las normas, analizamos el modelo de AEY en una población de agentes localizados en una red social. Dos propiedades que caracterizan las redes sociales humanas es el efecto de pequeño-mundo (esto es, el hecho de que dos personas cualesquiera estén conectadas a través de un número pequeño de saltos) y el alto clustering (alta probabilidad de tener amigos en común, o lo que es lo mismo, la alta probabilidad de que un amigo de un amigo sea a la vez amigo nuestro). En concreto, para modelar este efecto de forma simultánea, utilizamos un mecanismo de generación de redes aleatorias basado en el algoritmo de Watts y Strogatz (1998).

En (Santos et al., 2012) situamos a los agentes en una red de Watts-Strogatz y analizamos la influencia de la red en la evolución transitoria y asintótica del sistema. En concreto, descubrimos que la presencia de mayor clustering en la red propicia la aparición de equilibrios paritarios, mientras que en las redes de bajo clustering la emergencia de la norma discriminatoria se hace más frecuente. Además, observamos que en las redes que presentan alto clustering el tiempo necesario para que la norma paritaria se difunda por todo el sistema es mayor que en los casos en los que la red presenta bajo clustering.

No podemos olvidar que esta tesis doctoral queda enmarcada dentro del marco conceptual de la Teoría de la Organización. Por ello, se ha llevado a cabo un análisis de las distintas corrientes de pensamiento económico indicando los puntos en común y los puntos enfrentados entre dichas corrientes y esta tesis.

La Figura 1 muestra el marco de esta tesis doctoral, sus puntos de partida y sus herramientas. 


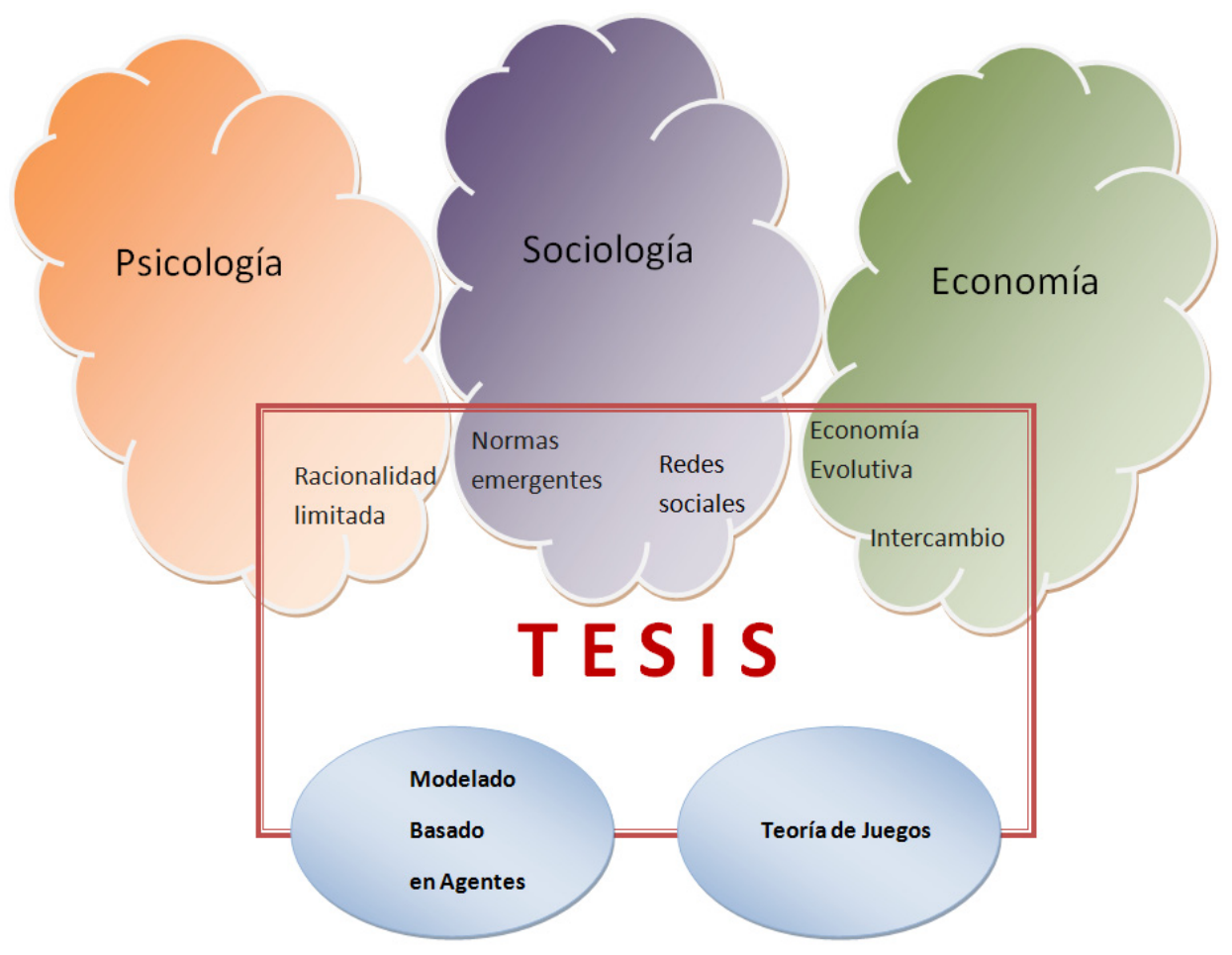

Figura 1. Marco de esta tesis doctoral. 


\subsection{EL GRUPO INSISOC}

INSISOC $^{2}$ (INgeniería de los SIstemas SOCiales) es un Grupo de Investigación de Excelencia de la Junta de Castilla y León compuesto por investigadores de la Universidad de Valladolid y de la Universidad de Burgos. Su objetivo es promocionar la investigación y el modelado del comportamiento de los sistemas sociales complejos desde la conducta de los agentes que lo componen, explorar y desarrollar metodologías en el campo del pensamiento sistémico y construir herramientas que faciliten su aplicación al estudio de los problemas complejos.

INSISOC surge como resultado del trabajo desempeñado por el profesor Dr. Cesáreo Hernández dirigiendo la tesis doctoral "Análisis e Ingeniería de las Instituciones Económicas. Una metodología basada en agentes", realizada por el Dr. Adolfo LópezParedes. Se considera como hito inicial del grupo la presentación del artículo "The Social Dimension of Economics and Multiagent Systems" por el Dr. López-Paredes y el Dr. del Olmo (1998).

El apoyo y asistencia de los profesores del Centre for Policy Modelling de la Manchester Metropolitan University, y, en particular, de su director, el profesor Scott Moss, fueron claves en los primeros pasos de INSISOC. Los resultados del grupo dirigido por el profesor Hernández se materializaron en las primeras tesis doctorales en nuestro país que incluyen el modelado basado en agentes en las ciencias sociales en general, y en la ciencia económica en particular, como una tercera vía en la investigación científica: (López Paredes, 2001; Pajares, 2001; Posada Calvo, 2005; Pascual Ruano, 2006).

El grupo INSISOC fue pionero en la publicación de los primeros manuales sobre modelado basado en agentes escritos en español. Entre ellos destaca el libro "Ingeniería de Sistemas Sociales. Diseño, Modelado y Simulación de Sociedades Artificiales de Agentes" (López-Paredes, 2004), que toma el nombre del grupo de investigación. Otros volúmenes publicados en castellano que han contribuido a la difusión de las actividades del grupo y a la internacionalización de sus actividades han sido: López Paredes (2001); Aguilera Ontiveros y López Paredes (2001); Aguilera (2002); Aguilera y López-Paredes 
(2004) o López Paredes y Hernández Iglesias (2008), en el que se recogen trabajos diversos que van desde la metodología general de modelado basado en agentes a aplicaciones en la gestión de recursos naturales en los más diversos ámbitos.

En la actualidad, INSISOC cuenta con más de 20 investigadores y es uno de los grupos de investigación en el campo con más presencia en el panorama nacional. En la Figura 2 se muestra un esquema de los campos de investigación actuales de este grupo.

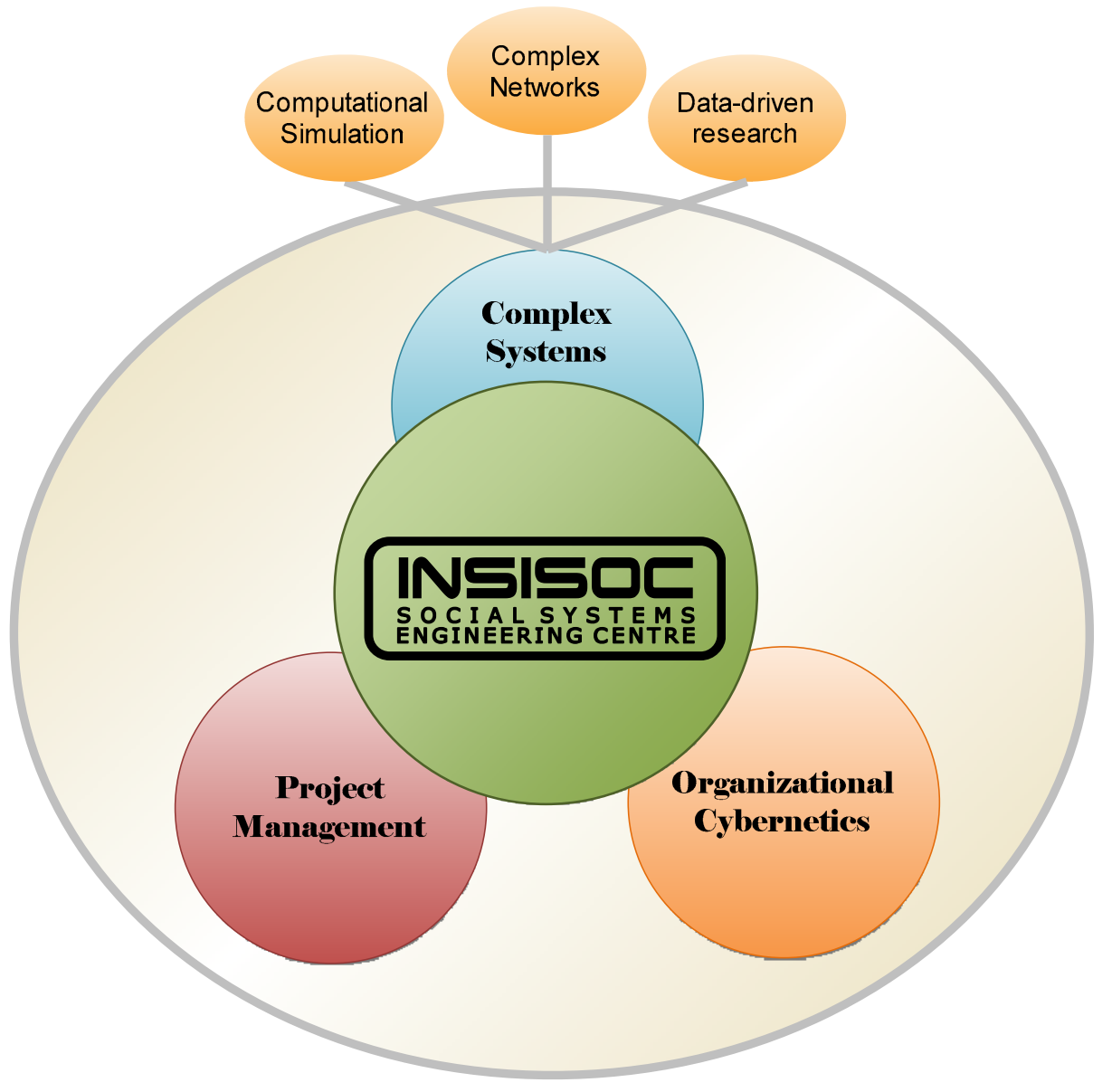

Figura 2. Líneas de investigación del grupo INSISOC.

En la actualidad, existen tres líneas de investigación dentro del grupo INSISOC: Sistemas Complejos, Project Management y Cibernética Organizacional. 
Este trabajo de investigación se enmarca dentro del campo de los Sistemas Complejos. Dentro de esta área de conocimiento, INSISOC aplica su conocimiento sobre modelado basado en agentes a la simulación computacional, las redes complejas y el datadriven research.

\section{- SIMULACIÓN COMPUTACIONAL}

Se ha empleado simulación computacional en diversos campos, tales como la teoría de juegos y la evolución de la cooperación (Galán \& Izquierdo, 2005; Galán Ordax, López Paredes \& del Olmo Martínez, 2005; Izquierdo \& Izquierdo, 2006; Izquierdo et al., 2007, 2008; Izquierdo, Izquierdo \& Gotts, 2008; Izquierdo \& Izquierdo, 2008; Pascual et al., 2009; Poza, Villafáñez \& Pajares, 2009; Poza et al., 2010; Izquierdo \& Izquierdo, 2011); el estudio de los mercados artificiales (Pascual Ruano et al., 2003; Pajares et al., 2003; Pascual \& Pajares, 2007; Pascual, Pajares \& López-Paredes, 2006; Lavios et al., 2006; López-Paredes et al., 2008; Hernández et al., 2008); imperfecciones de mercado (Izquierdo et al., 2006; Izquierdo \& Izquierdo, 2007). Dentro del ámbito económico, se procedió al estudio del comportamiento y la reproducción de agentes artificiales en los mercados regulados mediante subastas (Posada Calvo, Hernández Iglesias \& López Paredes, 2004; Posada Calvo, 2005; Posada Calvo, Hernández-Iglesias \& Lopez-Paredes, 2006; Posada, Hernández \& López-Paredes, 2006; Posada, 2006; Posada, Hernández \& López-Paredes, 2007; Posada \& López-Paredes, 2008; Posada, Hernández \& López, 2008; Villahoz, del Olmo Martínez \& Arauzo, 2010; FuentesFernández et al., 2010; Villafáñez \& Poza, 2010); la caracterización de los problemas de negociación bilateral (Hernández Iglesias \& López Paredes, 1999; López Paredes, 2001; López-Paredes, Hernández-Iglesias \& Gutiérrez Pajares, 2002) y la caracterización de comportamientos en dinámica industrial (Pajares, López \& Hernández, 2003; Pajares, Hernández-Iglesias \& López-Paredes, 2004; López-Paredes et al., 2008).

\section{- REDES COMPLEJAS}

Algunas publicaciones destacadas son las siguientes: Santos Martín, del Olmo Martínez \& Pajares Gutiérrez (2005); Santos, Galán \& del Olmo (2006); Izquierdo \& 
Izquierdo (2007); McAllister et al. (2009); Poza et al. (2010); Galán, Latek \& Rizi (2011); Poza et al. (2011); Santos, Olmo \& Pajares (2007); Santos et al., 2012).

\section{- DATA-DRIVEN RESEARCH}

Con la tesis del Dr. Galán (2007), el grupo INSISOC comenzó a aplicar el modelado basado en agentes a la gestión de recursos naturales, concretamente para tratar la complejidad de los de los múltiples factores que influyen en la gestión del agua doméstica en zonas metropolitanas emergentes. Posteriores trabajos en esta línea de investigación son: Galán, del Olmo \& López-Paredes (2008); Galán, López-Paredes \& del Olmo (2009); Galán Ordax (2007); Izquierdo et al. (2008); Galán et al. (2009); Izquierdo et al. (2009); Fuentes-Fernández et al. (2011).

En la Figura 3 se muestran los proyectos en los que ha participado y participa en la actualidad el grupo INSISOC. 


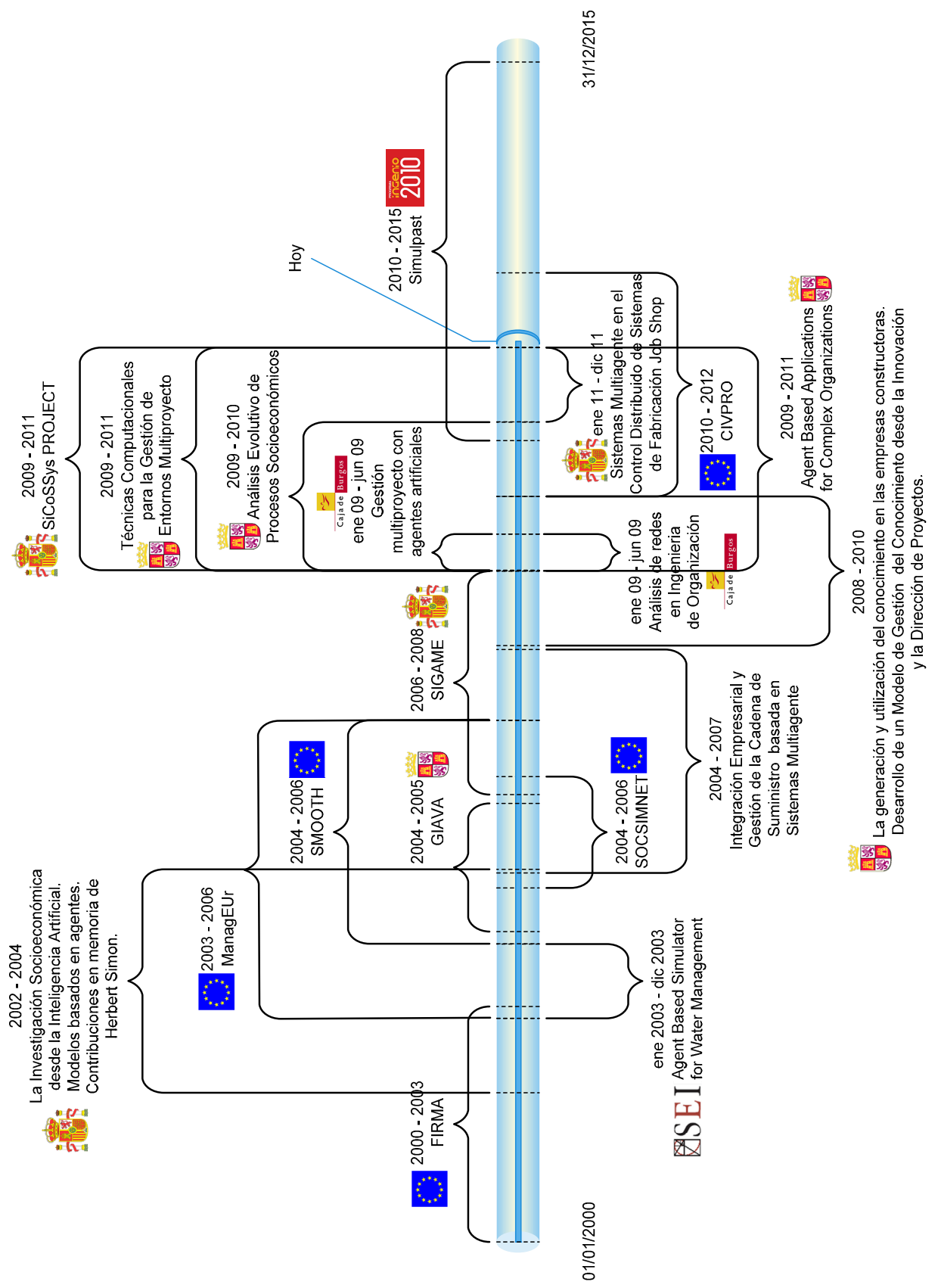

Figura 3. Proyectos en los que ha participado y participa en la actualidad el grupo INSISOC. Adaptado de (Galán Ordax, 2007). 


\subsection{UBICACIÓN DE LA INVESTIGACIÓN DENTRO DE LAS CORRIENTES DE PENSAMIENTO ECONÓMICO}

A pesar de que existen varios enfoques que tratan de explicar el papel de la empresa en la actividad económica, ninguna de estas teorías es excluyente. Estas teorías, por lo general, actúan de manera complementaria, explicando diversos aspectos de la empresa desde puntos de vista diferentes. Los límites que separan unas corrientes económicas de otras no son estrictos, por lo que existen ideas que son compartidas por varias corrientes. Como consecuencia, no resulta sencillo hacer una clasificación de estas corrientes de pensamiento.

Un criterio interesante para clasificar los distintos tipos de corrientes económicas consiste en distinguir si su enfoque es mecanicista o evolutivo (también llamado evolucionista $)^{3}$.

Según el enfoque mecanicista, se supone por hipótesis que los sistemas están en equilibrio y que existen ciertas leyes que relacionan las principales variables del sistema. Ante una perturbación, el sistema evoluciona hacia un equilibrio, ya sea éste el equilibrio previo u otro de los posibles equilibrios del sistema. Desde el punto de vista mecanicista, la labor del científico consiste, por tanto, en descubrir cuáles son estas leyes que dirigen el sistema hacia el equilibrio.

Por el contrario, el enfoque evolutivo no "impone" la existencia de este equilibrio ni de estas leyes inmutables. Según el razonamiento evolutivo (sea cual sea la ciencia a la que se aplique), se parte de los conceptos de herencia y selección (que contribuyen a mantener la homogeneidad del sistema) y de mutación (que contribuye a reducir la homogeneidad del sistema).

La Economía es una ciencia relativamente reciente. Cuando surgió, lo hizo con un enfoque mecanicista, "imitando" a otras ciencias existentes. La Economía Neoclásica

\footnotetext{
${ }^{3}$ Los enfoques mecanicista y evolutivo no son exclusivos de la Economía, sino que son aplicables a cualquier otra ciencia.
} 
hereda esta visión mecanicista del Universo, en la que todo se encuentra regulado por leyes matemáticas que empujan el sistema hacia puntos de equilibrio. Un ejemplo de razonamiento mecanicista en Economía lo tenemos en el equilibrio de producción de las empresas según la Economía Neoclásica: en un mercado de competencia perfecta, el equilibrio se alcanza cuando el nivel de producción de las empresas hace que el coste marginal de dicho nivel de producción sea igual al precio del bien en el mercado ${ }^{4}$.

Según el enfoque evolutivo, si bien existen factores que empujan a las empresas hacia la homogeneidad, como por ejemplo, el hecho de que la manera de operar en las empresas no cambia de un día para otro (concepto de herencia), también existen otros factores que empujan a las empresas hacia la diversidad, como por ejemplo la innovación (concepto de mutación). Además, en el mercado existen mecanismos que hacen que unas empresas alcancen el éxito y otras no (concepto de selección). Ideas evolucionistas en dinámica industrial son, por ejemplo, los fenómenos de path-dependence, o la concepción de la tecnología como el resultado de los esfuerzos de $\mathrm{I}+\mathrm{D}$ de la compañías (teoría del crecimiento endógeno).

Mientras que la Teoría Neoclásica asume que los agentes económicos tienen racionalidad ilimitada (poseen toda la información necesaria - y además saben utilizarla - para tomar las decisiones que maximizan su utilidad), la aproximación evolutiva en Economía supone la racionalidad limitada de los agentes económicos (lo que implica aprendizaje, comportamiento adaptativo y la posesión de una serie de competencias y habilidades). Además, la empresa es concebida como un agente procesador de conocimiento, en el que se genera, se trasforma y se produce conocimiento.

El criterio que utilizan algunos autores para distinguir entre unas corrientes de pensamiento y otras es el siguiente: ¿Cuáles son las fuentes de beneficio de las empresas? Según este criterio, en primer lugar tendríamos la Teoría Económica, dentro de la cual se integran la Economía Neoclásica, La Economía Industrial, la Nueva Economía Institucional (con la Teoría de los Derechos de Propiedad, la Teoría de la

${ }^{4}$ Sin embargo, en la vida real, una empresa tiene gran dificultad para conocer con exactitud cuál es su estructura de costes, por lo que aún le resultará más complicado conocer el coste marginal de los bienes producidos. Por lo tanto, en la práctica, a las empresas no les resulta fácil conocer cuál es el nivel de producción que maximiza su beneficio y que constituye un punto de equilibrio según la visión mecanicista de la Economía Neoclásica. 
Agencia y la Teoría de los Costes de Transacción) y la Teoría Evolucionista. En segundo lugar tendríamos las diversas teorías de la Administración (como, por ejemplo, el Taylorismo), y, en tercer lugar, el Enfoque Estratégico (con la Teoría de las Fuerzas Competitivas, el Conflicto Estratégico, la Teoría de Capacidades y Recursos, y la Teoría de Capacidades Dinámicas). Ver esquema en la Figura 4.

Según la Teoría Económica, la Organización de Empresas no sólo debe proporcionar normas que faciliten la asignación eficiente de los recursos dentro de la propia empresa, sino que debe, además, justificar la existencia de empresas y de otras formas organizativo-contractuales alternativas al mercado. A grandes rasgos, las principales aportaciones de la Teoría Económica son el análisis del entorno económico en el que se desenvuelven las empresas y el estudio de la naturaleza y la estructura básica de las mismas (Vázquez Bustelo, 2011).

Como ya mencionamos anteriormente, según la Economía Neoclásica, se asume que el ser humano posee racionalidad ilimitada. Además, según su punto de vista, todas las empresas pertenecientes a un mismo sector tienen el mismo comportamiento y pueden ser modeladas como una única empresa representativa. Es decir, la empresa es considerada como una "caja negra" con "producción" a su salida y con "factores productivos" (capital y trabajo) y "materias primas" a su entrada. Según esta teoría, el funcionamiento interno de la empresa no importa. Además, el bien que comercializan todas estas empresas es el mismo (hipótesis de bien homogéneo). En consecuencia, la Teoría Neoclásica tiene muchas dificultades para explicar por qué unas empresas tienen éxito y otras no.

La Economía Industrial, en cambio, con su paradigma ECR (estructura - conducta resultados), se aleja un poco de la Teoría Neoclásica y afirma que los mercados no son perfectos, si bien sigue considerando que los agentes sí son perfectos (es decir, tienen capacidad de razonamiento ilimitada). En otras palabras, la Economía Industrial estudia el comportamiento de unos agentes (racionales) en unos mercados imperfectos.

La Nueva Economía Institucional se caracteriza por una visión contractual de las relaciones internas en la organización y las existentes entre distintas empresas, analizando sus costes. Las tres subcorrientes de la Nueva Economía Institucional (Teoría de los Costes de Transacción, Teoría de la Agencia, Teoría de los Derechos de propiedad) tienen en común el descontento con el tratamiento que hace de la empresa la 
Economía Neoclásica (Kim \& Mahoney, 2005). La Teoría de los Costes de Transacción analiza los costes que ocasionan las distintas formas de gobierno de los intercambios y el predominio de unas formas organizativas sobre otras en determinados contextos institucionales (Vázquez Bustelo, 2011). El desarrollo de esta teoría se debe fundamentalmente a Oliver Williamson (2000), quien se basó en el trabajo seminal de Ronald Coase. La Teoría de la Agencia se centra en un análisis de los contratos entre agentes económicos y en el problema del control del comportamiento que surge en dichas relaciones contractuales. La Teoría de los Derechos de Propiedad se encarga del estudio del conjunto de normas costumbres y leyes que contribuyen a definir los modos de apropiabilidad, las formas de utilización y las reglas de intercambio de los recursos.

La Teoría de la Administración analiza cómo distintas consideraciones de gestión afectan a los resultados de la empresa.

El Enfoque Estratégico, como su nombre indica, se centra en encontrar qué estrategias son las que llevan a ciertas empresas al éxito. Según la Teoría de las Fuerzas Competitivas (Porter, 1980), los resultados de la empresa dependen fundamentalmente de cinco factores (la rivalidad de los competidores, la amenaza de nuevos competidores, la amenaza de productos sustitutivos, el poder negociador de los proveedores y el poder negociador de los clientes) que pueden aportar a la empresa ciertas ventajas competitivas. Para la Teoría de Recursos y Capacidades, cuyo máxima representante es Penrose (1959), la empresa es considerada como un conjunto de recursos y capacidades, tanto físicos (capital, máquinas, terrenos, etc.) como intangibles (patentes, marcas, reputación, know-how, tecnología, etc.) que proporcionan a cada empresa ciertas ventajas competitivas. Por otro lado, la Teoría de Capacidades y Recursos, con David J. Teece como máximo representante, va más allá y afirma que la posesión de recursos y capacidades estratégicas no garantiza el mantenimiento de las ventajas competitivas a medio/largo plazo, sino que éstas deben ser renovadas (mediante la innovación) para adaptarse a los cambios en el entorno. 


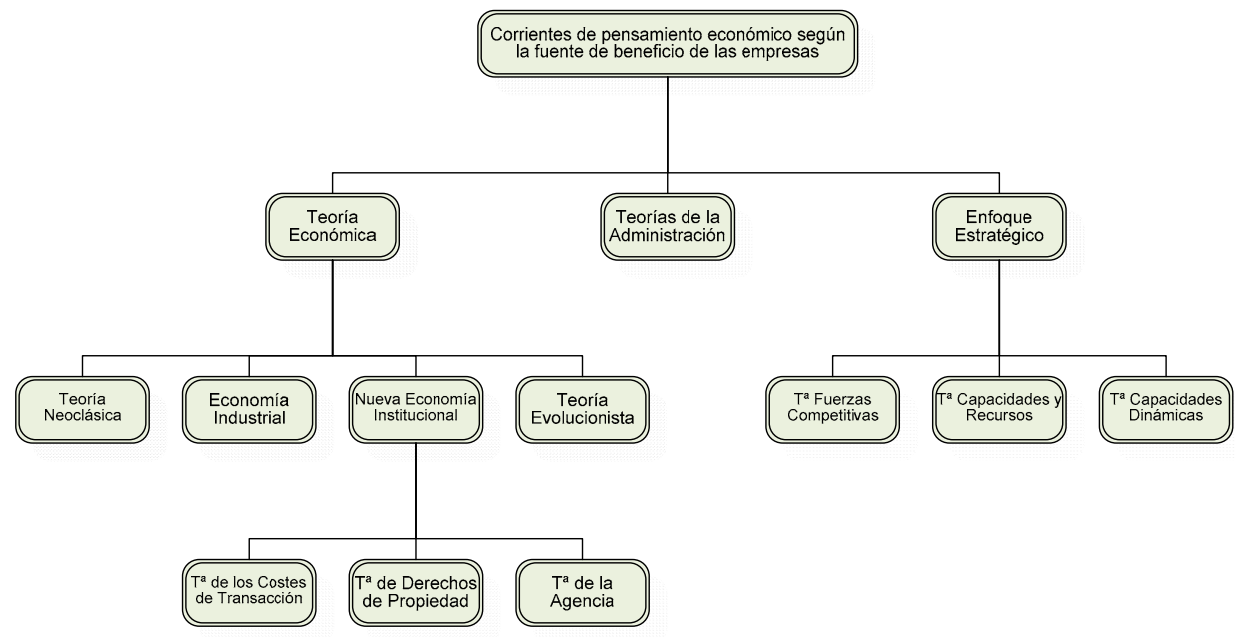

Figura 4. Una posible clasificación de las corrientes de pensamiento económico.

Existen otros criterios para clasificar las corrientes de pensamiento económico, aparte de las expuestas anteriormente. Por ejemplo, Mahoney (2005), hace esta clasificación en función de la estrategia, y distinguen cinco corrientes: Behavioral Theory of the Firm (¿Cómo pueden las empresas operar de una manera eficiente?), Teoría de los Costes de Transacción (¿Cómo pueden las empresas reducir sus costes?), Teoría de los Derechos de Propiedad (¿Cómo pueden las empresas crear y distribuir riqueza?), Teoría de la Agencia (¿Cómo pueden las empresas alinear los intereses individuales?) y Teorías basadas en recursos / capacidades dinámicas: (¿Cómo pueden las empresas adquirir, desarrollar y utilizar recursos para aumentar la probabilidad de supervivencia y de crecimiento rentable?).

Como vemos, además de que los límites de las distintas corrientes no están rigurosamente definidos, la clasificación de las distintas corrientes varía según los criterios de cada autor. Nuestro trabajo no se situará inamoviblemente en una sola corriente, sino que tomará ideas y conceptos de varias de ellas. Aun así, indicaremos a continuación, cuáles de las distintas corrientes son más cercanas o más lejanas a nuestro trabajo.

La corriente de pensamiento que queda más alejada de la filosofía de esta tesis doctoral es la Teoría Neoclásica debido fundamentalmente a su hipótesis de racionalidad ilimitada. Esta investigación, de hecho, se aleja de esta suposición. Si bien sí se asume que los agentes intentan maximizar su beneficio, en nuestros modelos se considera que, 
aunque lo intentan, muchas veces no saben cómo hacerlo, tomando elecciones que no siempre son las óptimas. Además, en nuestros modelos, también hay cabida para la exploración.

La corriente de pensamiento más acorde con esta investigación es la Teoría de los Costes de Transacción, puesto que en ella se plantean cuáles son los mecanismos por los cuales emergen ciertas normas que acaban institucionalizándose dando lugar a contratos estándar (Young, 1998). De hecho, la emergencia de normas mitiga los costes de transacción (información, negociación y garantía). Retomemos el ejemplo de la norma establecida en cuanto al reparto de excedente entre bufete de abogados y cliente en EEUU (reparto de excedente 2/3 - 1/3), comentado en la introducción a este documento. Una vez la norma se ha difundido en la sociedad, no necesitamos informarnos sobre qué reparto se hace en distintos bufetes, ya que todos ellos seguirán la misma norma de reparto, lo cual supone una reducción de costes de información. Además, no tiene sentido negociar otro reparto distinto al de la norma establecida, ya que, si el reparto resultara menos favorable para nosotros, no tendríamos ningún problema en encontrar otro bufete que siguiera la norma $2 / 3-1 / 3$, lo cual implica una reducción de costes de negociación. Finalmente, una vez establecida la norma, suelen surgir contratos que dan soporte legal a la misma, lo cual se traduce en una reducción en los costes de garantía. 



\section{MARCO METODOLÓGICO}

\subsection{MODELADO BASADO EN AGENTES}

\subsubsection{INTRODUCCIÓN}

El modelado basado en agentes $\left(\mathrm{ABM}^{5}\right)$ ha cobrado gran importancia desde hace algunas décadas en campos como la Sociología (Conte, 1997; Gilbert \& Conte, 1995; Gilbert \& Troitzsch, 1999; Suleiman, Troitzsch \& Gilbert, 2000; López-Paredes, 2004), Ciencias Políticas (Axelrod, 1997; Johnson, 1999), la Economía (Arthur, Durlauf, \& Lane, 1997; Tesfatsion, 2002; López et al. 2004), la Biología (Resnick, 1997; Bauer, Beauchemin \& Perelson, 2009), la Antropología (Wolfe, 2003; Lansing, 2003) o la gestión de recursos y la Ecología (Bousquet \& Le Page, 2004; Hare \& Deadman, 2004; Janssen, 2003; Galán et al. 2009).

Tanto es así que esta herramienta se ha posicionado entre los dos paradigmas tradicionales de modelado científico, el que han seguido tradicionalmente las Ciencias Naturales y el seguido tradicionalmente por las Ciencias Sociales, al combinar ventajas de ambas aproximaciones (López-Paredes, 2004).

Por un lado, el modelado en las Ciencias Naturales ha estado basado en ecuaciones matemáticas (que le confieren rigor formal), mientras que el modelado en las Ciencias Sociales ha estado basado tradicionalmente en el lenguaje natural (que le aporta realismo y expresividad) (Moss, 1999). El modelado basado en agentes combina tanto formalidad como rigor, al mismo tiempo que minimiza las desventajas los paradigmas de modelado tradicionales (fuertes hipótesis en caso de las Ciencias Naturales y ambigüedad en el caso de las Ciencias Sociales).

Centrándonos en la Economía, ésta ha sido modelada tradicionalmente con ecuaciones matemáticas. Para poder modelar una realidad tan compleja mediante expresiones

${ }^{5}$ Agent-Based Modeling 
matemáticas relativamente sencillas, fue necesario hacer fuertes hipótesis sobre el comportamiento del ser humano y de las empresas a la hora de tomar decisiones económicas. Estas hipótesis se tradujeron en que, a la hora de tomar decisiones económicas, todos los seres humanos y todas las empresas actúan siguiendo un mismo comportamiento representativo. Esto introdujo una homogeneidad en el sistema que no se observaba en el mundo real. Por otro lado, modelar el comportamiento humano con lenguaje natural, al que tradicionalmente han recurrido la Sociología y la Psicología introducía ambigüedad y dificultaba el análisis formal.

En términos generales, el modelado basado en agentes consiste en la construcción de un modelo mediante un ordenador que permita observar el comportamiento emergente resultado de la interacción entre agentes software que interactúan entre sí según unas reglas de comportamiento. A los agentes software se les asignan los distintos comportamientos observados en el ser humano por las Ciencias Sociales (realismo) pero formalizados computacionalmente (rigor). Por consiguiente, el modelado basado en agentes se posiciona en un punto intermedio entre el paradigma de modelado científico tradicional de las Ciencias Naturales, basado en ecuaciones matemáticas (y, por tanto, formal) y el enfoque de modelado tradicional de las Ciencias Sociales, típicamente basados en el lenguaje natural (y, por tanto, realista).

En la siguiente sección daremos una descripción más formal del modelado basado en agentes.

\subsubsection{DEFINICIÓN DE AGENTE Y MODELADO}

En primer lugar, antes de definir formalmente el modelado basado en agentes, comenzaremos definiendo qué es un agente y exponiendo en qué consiste, en general, el modelado.

A pesar de no existir una definición universalmente aceptada, una definición de agente a nuestro entender bastante aceptada es la siguiente: 
Un agente es un hardware o (más frecuentemente) una entidad software que, situada en un entorno, es capaz de exhibir un comportamiento autónomo flexible, con el propósito de conseguir los objetivos para los que fue diseñado.

\section{(Wooldridge \& Jennings, 1995)}

Un modelo es una abstracción de una realidad en la cual se seleccionan los elementos de la misma que se consideran relevantes para la situación que se está estudiando. En ocasiones se hacen hipótesis sobre elementos desconocidos, o bien se hacen simplificaciones sobre ciertos aspectos (Galán et al., 2009).

Si bien el modelado posibilita el estudio de ciertos fenómenos de la naturaleza que no podrían llevarse a cabo sin las aproximaciones y simplificaciones que se hacen en el momento de modelar, se ha de ser consciente de las aproximaciones efectuadas y de su posible influencia en las conclusiones que se extraigan del modelo.

El modelado basado en agentes puede definirse como:

"Un método computacional que permite al investigador crear, analizar y experimentar con modelos compuestos por agentes que interactúan con el entorno".

(Gilbert, 2008)

En esencia, este enfoque consiste en crear un programa de ordenador en el que las entidades identificadas en el sistema objetivo (y sus interacciones) son representadas por objetos software (los agentes) que interactúan entre sí dentro de un entorno virtual (Figura 5). La idea básica consiste en establecer una correspondencia directa entre los actores y los agentes de tal manera que la observación de la evolución del programa en el mundo virtual pueda ayudarnos a entender el sistema modelado (Galán, LópezParedes, \& Del Olmo, 2009). 


\section{Target System Agent based model}

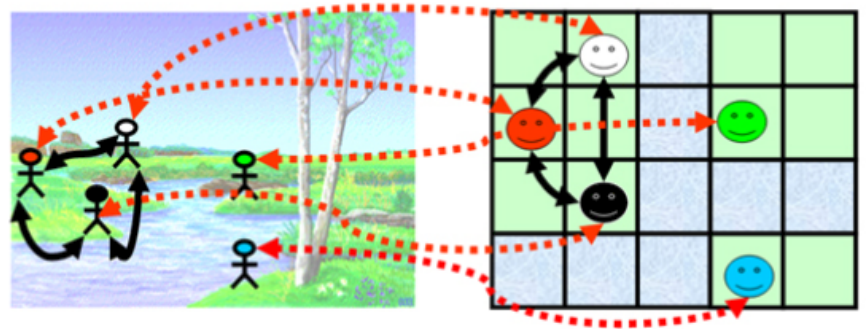

Entities

Agents

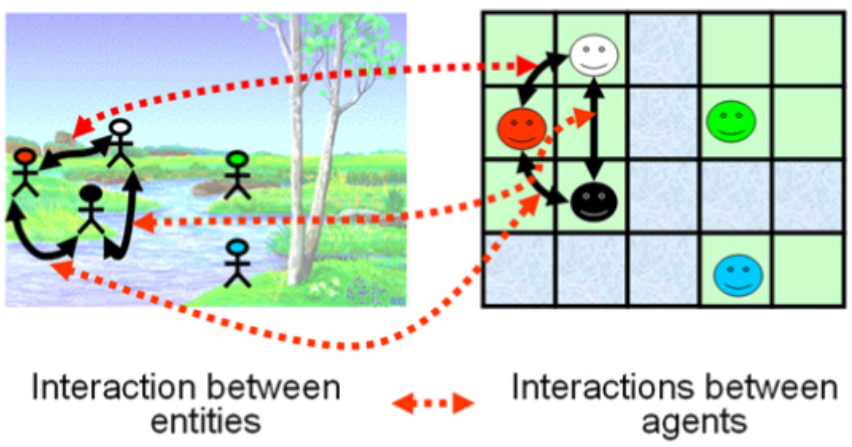

Figura 5. Relación entre entidades en la realidad; relaciones correspondientes entre agentes en el modelo basado en agentes (Edmonds, 2001).

\subsubsection{CARACTERÍSTICAS DEL MODELADO BASADO EN AGENTES}

Lo que diferencia a unos paradigmas de modelado de otros es la manera de construir las

abstracciones de la realidad observada. Una de las principales ventajas del modelado basado en agentes (y que le distingue del resto de paradigmas de modelado) es la posibilidad de establecer una correspondencia más directa entre entidades (y sus interacciones) del sistema a modelar y los agentes (y sus interacciones) en los modelos (Edmonds, 2001), tal como se observa en la Figura 5. 
Como ya se ha visto, el modelado basado en agentes combina las ventajas del paradigma de modelado seguido tradicionalmente por las Ciencias Naturales (rigor) y del paradigma de modelado seguido por las Ciencias Sociales (realismo). Esto permite una descripción formal (y aun así natural) del sistema objetivo, posibilita el modelado de la heterogeneidad, facilita la representación del entorno y la manera en la que los agentes interactúan con él, permite estudiar las relaciones bidireccionales entre los individuos y los grupos, y permite capturar el comportamiento emergente (Galán \& Izquierdo, 2005; Axtell, 2000; Bonabeau, 2002; Epstein, 1999).

Por otro lado, el modelado basado en agentes, como tercera vía de modelado, minimiza las principales desventajas de los dos paradigmas tradicionales: hipótesis y simplificaciones en la caso de las Ciencias Naturales y ambigüedad del lenguaje en el caso de las Ciencias Sociales.

No obstante, el modelado basado en agentes presenta una desventaja: los modelos resultantes son, en general, difícilmente tratables matemáticamente, por lo que debe recurrirse a la simulación computacional para su análisis (Galán Ordax, 2007; Galán \& Izquierdo, 2005). De ahí que hoy en día se utilicen las expresiones "modelado basado en agentes" y "simulación basada en agentes" como sinónimo (Galán et al., 2009).

Sin embargo, es precisamente la simulación computacional la que nos permite observar fácilmente el comportamiento emergente fruto de la interacción entre los diversos agentes que constituyen el modelo.

\subsubsection{MODELADO BASADO EN AGENTES Y COMPORTAMIENTO EMERGENTE}

A pesar de que en un modelo basado en agentes, a éstos les es asignado un patrón de comportamiento a nivel individual, en muchas ocasiones el comportamiento social resultante de la interacción entre los agentes tiene poco que ver con el comportamiento que podría esperarse a partir de los patrones de comportamiento individuales. 
Un claro ejemplo de este comportamiento emergente es el Modelo de Segregación Social de Schelling ${ }^{6}$ (1978), considerado por muchos autores como el primer modelo basado en agentes (López Paredes, 2011), a pesar de que, en su origen, no se trataba de un modelo computacional, sino que fue desarrollado en un tablero de damas y monedas (Izquierdo et al., 2009). En este modelo, se parte de una población de agentes artificiales, donde la mitad corresponde a agentes de una raza y la otra mitad a agentes de otra raza. Inicialmente, los individuos de ambas razas se encuentran mezclados y distribuidos aleatoriamente. El patrón de comportamiento asignado a los agentes es el siguiente: cada individuo está satisfecho con el lugar geográfico que ocupa siempre que el número de vecinos de raza diferente a la suya no supere cierto umbral. En caso de que este umbral se supere, los individuos se desplazan a una nueva posición geográfica. El proceso continúa hasta que todos los agentes alcanzan una posición en la que el número de vecinos de raza distinta a la suya sea inferior a este umbral. Mediante simulación, Edmonds y Hales (2005) comprobaron que, incluso cuando los valores de este umbral son grandes (es decir, cuando la población es tolerante y está satisfecha con una proporción elevada de vecinos de raza distinta a la suya), la interacción de los agentes da como resultado una segregación social, en la que los individuos de la misma raza tienden a agruparse. La Figura 6 muestra esta situación. En la parte izquierda se muestra la situación inicial, con una población de 2.000 agentes (1.000 de cada raza), distribuidos aleatoriamente. La figura derecha muestra la situación final, donde los agentes de distintas razas se han agrupado. El umbral empleado es 30\% (es decir, para que los agentes estén satisfechos basta con que 3 de cada 10 vecinos sean de su misma raza). Como vemos, resulta destacable que el comportamiento individual de los agentes, programados para ser, aparentemente, tolerantes, no hacía pensar la emergencia de segregación en la población y aun así, la segregación se produce para niveles altos del parámetro de tolerancia.

Thomas Schelling fue el primer economista que mostró explícitamente cómo las decisiones tomadas a nivel microscópico evolucionan hasta alcanzar patrones reconocibles a nivel macroscópico (Young, 1998).

${ }^{6}$ Debido a que Sakoda elaboró un modelo similar al de Schelling de manera simultánea, en ocasiones este modelo recibe el nombre de Schelling-Sakoda. 


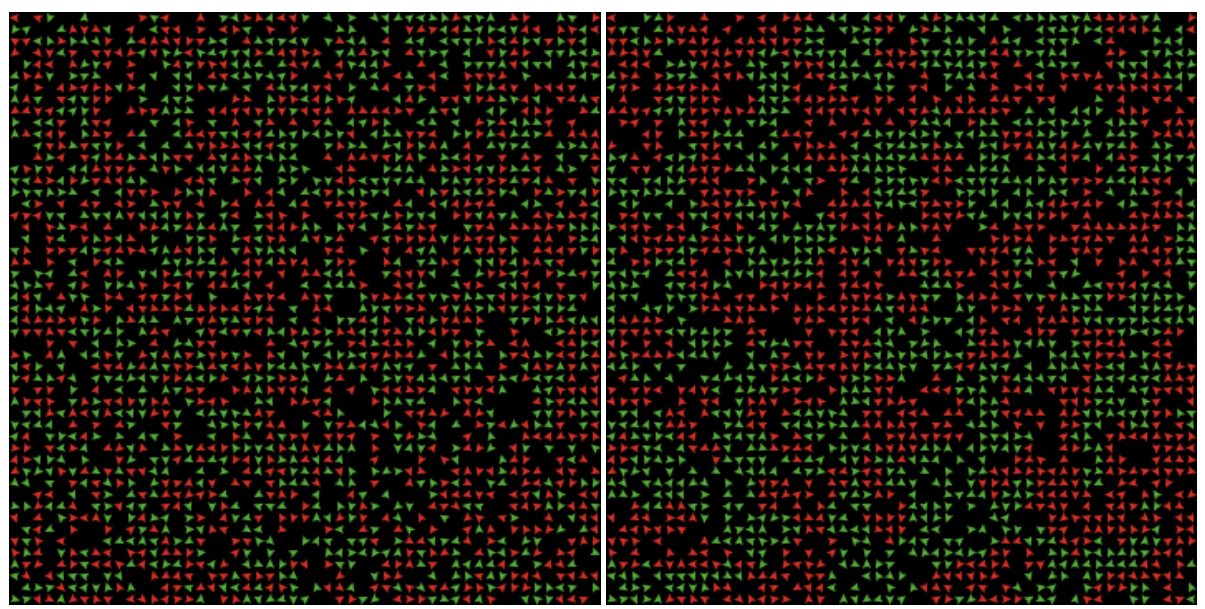

Figura 6. ABM del Modelo de Segregación de Schelling programado en Netlogo (Wilensky, 1999).

A la izquierda, situación inicial (distribución aleatoria de los agentes); a la derecha, situación de los agentes tras la interacción (segregados por raza). El color de los agentes indica la raza a la que pertenecen.

Como conclusión, podemos decir que a partir de la micro-definición del comportamiento de los agentes, el macro-comportamiento emergente no siempre resulta evidente. Mediante simulación, el modelado basado en agentes permite observar la aparición o ausencia de estos comportamientos emergentes, al mismo tiempo que facilita el análisis de las razones que llevan a la emergencia de estos macro comportamientos.

\subsubsection{BUENAS PRÁCTICAS EN LA CONSTRUCCIÓN DE MODELOS BASADOS EN AGENTES}

A pesar de las numerosas ventajas vistas hasta el momento, desafortunadamente, el modelado basado en agentes también presenta algunos inconvenientes.

En primer lugar, a la hora de escribir el código sobre el que se apoyará el modelo, resulta muy difícil no introducir bugs (Galán et al., 2009). Los bugs son fragmentos de código que no realizan lo que el programador esperaba y que provocan que el programa 
(y, por tanto, el modelo) funcione de una manera inadecuada. Por ejemplo, en la replicación del Modelo de Clases que se ha llevado a cabo en esta investigación, los agentes son emparejados al azar y juegan una única vez en cada ronda. Si, debido a un fallo en el código del programa, ocurriera que en alguna ronda algún agente no jugara o bien jugara más de una vez, el programador estaría introduciendo un bug en el código del programa.

En segundo lugar, cuando la simulación muestra un resultado llamativo, no resulta trivial averiguar si éste se debe a un fallo de programación o bien a que el modelo se comporta realmente así. Retomando el ejemplo anterior, si nuestro código contuviera este bug, esto podría originar que atribuyéramos erróneamente el resultado de la simulación al comportamiento del modelo cuando probablemente el responsable sea este error en el código que había pasado desapercibido.

En tercer lugar, aun cuando nuestro código no contenga errores, puede ocurrir que nuestro modelo contenga artefactos. Los artefactos pueden aparecer en el diseño de un modelo cuando las asunciones que se hacen de manera arbitraria porque se piensa que no influyen en los resultados, finalmente sí lo hacen (Galán et al., 2009; Galán \& Izquierdo, 2005). Si, por ejemplo, en un modelo se toma la decisión de no aleatorizar el orden en el que los agentes toman decisiones porque se asume que este orden no influye estaríamos introduciendo un artefacto si se introducen sesgos en favor de los que toman las decisiones en primer lugar, lo cual invalidaría los resultados. Por tanto, cuando aparecen artefactos, se corre el riesgo de malinterpretar los resultados de la simulación (Edmonds, 2009).

Ante la posibilidad de que se produzcan en nuestro modelo algunas de las tres circunstancias descritas anteriormente, la replicación tiene una importancia fundamental en el modelado basado en agentes (Axelrod, 1997; Edmonds \& Hales, 2005; Axtell et al., 1996; Edmonds \& Hales, 2003). Mediante la replicación, se reconstruye un modelo ya estudiado, utilizando para ello otro lenguaje de programación, de tal forma que se pueda comprobar si las conclusiones extraídas a partir del modelo original son consecuentes con las que se obtienen de la réplica del modelo. Por esta razón, nuestro trabajo de investigación comenzó con la replicación del Modelo de Clases de Axtell, Epstein y Young (2000) y no fue hasta cuando comprobamos que los resultados que habíamos obtenido con nuestra réplica del modelo eran consecuentes con los obtenidos en el modelo original cuando comenzamos a trabajar en las extensiones del modelo. 
En Galán et al. (2009) se describe una propuesta de elaboración de un modelo basado en agentes, con una descripción de las distintas fases y los distintos roles implicados para evitar, en la medida de lo posible, la introducción de errores y artefactos. Existen algunos intentos muy interesantes con INGENIAS (Pavón \& Gómez-Sanz, 2003) para utilizar lenguajes visuales y de modelado como lenguajes de programación en lugar de simplemente como lenguajes de modelado (Sansores \& Pavon, 2005; Sansores, Pavón \& Gómez-Sanz, 2006). Estos esfuerzos ayudan a sistematizar el paso desde el modelo ejecutable hacia la implementación (además en diferentes plataformas de simulación).

Otros dos conceptos asociados al modelado basado en agentes muy presentes en la literatura son la validación y la verificación. La validación es un proceso mediante el cual se evalúa cómo de útil es un modelo para la finalidad que llevó a su creación. Por otro lado, el proceso de verificación ${ }^{7}$ consiste en comprobar si el modelo resultante se comporta de la manera que tenían en mente sus diseñadores e implementadores (es decir, la verificación es el proceso por el cual se buscan errores).

Por ejemplo, para validar nuestras extensiones del Modelo de Clases, deberíamos plantearnos si este modelo es representativo del comportamiento social que estamos planteando. En el modelo de partida se asumía que los agentes se emparejaban de manera aleatoria, sin tener en cuenta ni su posición espacial ni las posibles relaciones entre agentes. Sin embargo, en una situación real, las personas tienden a relacionarse más frecuentemente con personas cercanas que con personas que se encuentran alejadas físicamente. De ahí nuestra extensión del modelo en el que situamos a los agentes en un grid regular de manera que éstos sólo pueden interactuar con sus vecinos (Poza et al., 2011). Por otro lado, las personas mantienen relaciones constituyendo redes sociales. De ahí nuestra siguiente extensión del modelo en la que hacemos que los agentes formen parte de redes sociales (Santos et al., 2012).

${ }^{7}$ La verificación, en algunas ocasiones, recibe el nombre de validación interna (Sansores and Pavon, 2005). 


\subsubsection{LA DIFICULTAD DE MODELAR EL COMPORTAMIENTO HUMANO}

En un modelo basado en agentes debe prestarse especial atención a cómo se define el comportamiento de los agentes puesto que esto no es una tarea sencilla. Tradicionalmente, la Teoría Económica ha considerado que los seres humanos poseen racionalidad ilimitada. Esto implica asumir que, a la hora de tomar decisiones económicas, los individuos disponen de toda la información necesaria, todas las habilidades requeridas para procesar esta información y, además, sin restricciones temporales. Por consiguiente, la hipótesis de racionalidad ilimitada implica que los individuos no tienen problemas para tomar la decisión óptima (es decir, aquella decisión que maximiza su utilidad).

Sin embargo, desde hace algunas décadas, la idea de la racionalidad limitada ha ido cobrando fuerza, especialmente a partir de los trabajos de Herbert Simon y Daniel Kahneman ${ }^{8}$. Según este punto de vista, la racionalidad del ser humano está limitada por tres dimensiones: la información disponible, la limitación de la mente de cada individuo (es decir, carencia de habilidad y recursos necesarios para tomar la decisión correcta) y el tiempo disponible para tomar la decisión (Simon, 1957). Esto quiere decir que los individuos no utilizan reglas de optimización estrictas para tomar decisiones, sino que emplean heurísticas que pueden no llevar a resultados óptimos, sino que conducen a resultados simplemente satisfactorios, debido a los costes de deliberación que el empleo de reglas de optimización complejas podría acarrear.

Kahneman contribuyó a la Ciencia Económica con su Teoría de las Perspectivas (1979) según la cual, cuando los seres humanos toman decisiones en entornos de incertidumbre, se alejan de los principios básicos de probabilidad y recurren a heurísticos. Según esta teoría, la aversión al riesgo de los individuos puede presentar asimetría en función de si la decisión tiene por objetivo maximizar ganancias o minimizar pérdidas, siendo la aversión al riesgo inferior en el segundo caso.

\footnotetext{
${ }^{8}$ Simon recibió el premio Nobel de Economía en 1978. También recibieron conjuntamente este galardón Kahneman y Vernon Smith en el año 2002.
} 
Posteriormente, Ariel Rubinstein (1998) propuso modelar la racionalidad limitada especificando explícitamente los procedimientos de toma de decisión.

Dentro del campo del modelado basado en agentes, Young (1998) demuestra que las dinámicas evolucionistas pueden conducir a un sistema en el que los agentes no tienen racionalidad ilimitada a los mismos estados de equilibrio que se alcanzan cuando los agentes sí disponen de ella. Young considera que los agentes no son completamente 'irracionales', sino que toman sus decisiones considerando cómo se comportan los otros agentes del sistema. El comportamiento de los agentes se actualiza endógenamente a partir de la información que van recibiendo del sistema. Young superaba de esta forma las principales críticas de Simon a los trabajos de racionalidad limitada en Teoría de Juegos de Rubinstein (Ariel Rubinstein, 1998: 187-190) recurriendo al uso de modelos basados en agentes (Schelling 1971, 1978).

A pesar de que en el Modelo de Clases (Axtell, Epstein \& Young, 2000), punto de partida de esta tesis doctoral, se asume que los agentes toman decisiones según una regla de comportamiento compleja, una de las contribuciones de nuestra investigación fue comprobar que cuando a los agentes se les dota de reglas de comportamiento heurísticas, los equilibrios que se alcanzan en el sistema son los mismos que cuando los agentes usan la regla de decisión empleada en el modelo original (véase sección 3.3.3).

${ }^{9}$ Otros autores (Epstein and Axtell, 1996; Gilbert and Troitzsch, 1999) coinciden al señalar los modelos de segregación de Schelling como el origen del Modelado y Simulación Basados en Agentes. 


\subsection{TEORÍA DE JUEGOS}

\subsubsection{INTRODUCCIÓN}

La Teoría de Juegos es una rama de las Matemáticas que estudia la toma de decisiones interdependientes. Es decir, aquellas situaciones en las que el resultado de la interacción entre individuos no sólo depende de la decisión tomada por cada uno de ellos individualmente, sino del par de estrategias tomadas por todos ellos en su conjunto.

Un ejemplo sencillo de situación que podría modelarse como un juego es la siguiente: dos personas que se cruzan al mismo tiempo para atravesar una puerta. ¿Quién pasará primero? Cada persona tiene dos posibles opciones de comportamiento (que llamaremos estrategias): pasar primero o ceder el paso. Esta situación tiene tres posibles resultados, en función del conjunto de las decisiones tomadas por los dos agentes implicados: el primero, que una persona pase primero y otra después (como resultado de que una persona decide pasar primero y la otra decide ceder el paso); el segundo, que las dos personas choquen al tratar de pasar por la puerta al mismo tiempo (porque las dos personas han decidido pasar primero); y tercero: ninguna de las dos personas pase (como resultado de que ambas personas deciden ceder el paso).

De los tres resultados posibles que tiene la situación que hemos modelado como un juego, ¿cuál sería el más deseable? Depende de a quién se lo preguntemos ${ }^{10}$. Si le preguntamos a cada una de las personas por separado, probablemente nos dirían que, para ellas, el mejor resultado sería que pasaran ellos mismos primero, ya que así la persona que pasa primero estaría ahorrando tiempo a costa de hacérselo perder a la segunda. Sin embargo, desde un punto de vista externo (suponemos que las dos personas nos son indiferentes), podríamos opinar que el mejor resultado desde el punto de vista social es aquél en el que una de las dos personas pasa y la otra cede el paso (sin importar cuál de las dos personas toma una decisión y cuál toma la otra).

${ }^{10}$ Hemos supuesto que cada persona prefiere su propio bien ante el bien de la sociedad. 
Aunque el ejemplo anterior resulte muy sencillo, recoge los tres elementos que forman parte de un juego. Desde un punto de vista más formal, decimos que un juego es una abstracción formal de una interacción social donde:

a) existen dos o más individuos (jugadores) que toman decisiones,

b) cada jugador tiene dos o más formas de actuar (llamadas acciones o estrategias), y

c) el resultado de la interacción depende de la decisión tomada por todos los jugadores (Izquierdo, Izquierdo \& Vega-Redondo, 2012).

El uso de la Teoría de Juegos no se limita a casos tan sencillos como el ejemplo anterior. Es muy utilizada en las Ciencias Sociales en campos como las carreras armamentísticas, posturas en política internacional, etc. Pero lo que a nosotros nos interesa realmente son las aplicaciones de la Teoría de Juegos en Economía: precio de un nuevo producto cuando otras empresas tienen productos similares, decidir si pujar o no en una subasta, etc. En todas estas situaciones, el resultado final no dependerá solamente de nuestra propia decisión, sino también de las decisiones adoptadas por los demás, razón por la cual la Teoría de Juegos constituye una buena herramienta para poder analizar dichas situaciones.

Muchos autores sitúan el origen de la Teoría de Juegos en 1944, con la publicación del libro "The Theory of Games Behaviour" por John Von Neumann y Oskar Morgenstern (Ross, 2011), aunque algunas de las ideas de la Teoría de Juegos ya habían sido anticipadas en el siglo XIX, por los economistas Cournot y Edgeworth (Contreras et al., 2002).

\subsubsection{LA MATRIZ DE PAGOS}

Como hemos visto previamente, el resultado de un juego depende del conjunto de las decisiones tomadas por los individuos participantes. Un elemento fundamental para representar los posibles resultados del juego en función de las distintas combinaciones de estrategias es la matriz de pagos. 
Ilustraremos el significado de la matriz de pagos con el dilema del prisionero, un juego de cooperación bien conocido.

La filosofía de este juego es la siguiente: dos ladrones han sido capturados y la policía no tiene evidencias suficientes para condenar o liberar a los detenidos, por lo que están siendo interrogados. Los interrogatorios se están llevando a cabo de tal manera que no existe ninguna posibilidad de comunicación entre los dos prisioneros. Los prisioneros pueden elegir entre dos estrategias, cooperar (C) o no cooperar (D). En este juego existen tres posibles resultados:

- Ambos prisioneros cooperan: los dos niegan haber cometido el crimen (C, C) y son condenados únicamente a un mes de prisión.

- Ninguno de los dos prisioneros coopera: los dos se traicionan mutuamente (D, D), y ambos son condenados a 3 meses de prisión.

- Un prisionero coopera negando haber cometido el crimen, y el otro no coopera admitiendo haber cometido el crimen (C, D) ó (D, C). Al prisionero que ha admitido cometer el crimen lo dejan libre y al que lo ha negado lo condenan a 12 meses de prisión.

Para representar los distintos resultados del juego, confeccionamos la siguiente matriz de pagos (Tabla 1):

Jugador 2

Jugador 1

\begin{tabular}{|l|c|c|}
\cline { 2 - 3 } \multicolumn{1}{c|}{} & Cooperar $(\mathrm{C})$ & No Cooperar $(\mathrm{D})$ \\
\hline Cooperar $(\mathrm{C})$ & 1,1 & 12,0 \\
\hline No cooperar $(\mathrm{D})$ & 0,12 & 3,3 \\
\hline
\end{tabular}

Tabla 1. Matriz de pagos del juego del dilema del prisionero.

La matriz de pagos recoge los posibles resultados del juego en función de cada combinación de estrategias elegida por los agentes. El hecho de tener esta información resumida en una tabla nos ayuda a analizar el resultado del juego.

Aunque en este caso concreto el valor de los pagos (payoffs) que introducimos en la matriz de pagos tiene un significado real (meses de condena), en general, el valor concreto del pago representa simplemente de forma ordinal las preferencias de los jugadores por cada uno de los resultados. Salvo que se indique lo contrario, el hecho de que los pagos asociados a una estrategia sean mayores que los pagos asociados a otra estrategia, simplemente significa que la primera estrategia es preferible para el agente 
ante la segunda, pero su valor concreto no suele ser representativo en términos cardinales.

En realidad, la mayoría de los pagos en la Teoría de Juegos Clásica son interpretados como utilidades de Neumann y Morgenstern, lo que significa que los pagos expresan la actitud de los jugadores ante el riesgo, de tal manera que puede emplearse la teoría de la utilidad esperada para evaluar las distribuciones de probabilidad de cada uno de los posibles resultados del juego ${ }^{11}$ (Izquierdo, Izquierdo \& Vega-Redondo, 2012).

¿Cuál será el resultado del juego? Por un lado, como observadores, nos damos cuenta de que la mejor situación conjunta para los dos jugadores es que ambos cooperen (C, C), pues, de esta manera, la suma de ambas penas es la menor de los cuatro posibles resultados del juego. Pero, por otro lado, si nos ponemos en el papel de uno de los prisioneros, sentiríamos una elevada tentación para traicionar a nuestro compañero (no cooperar, D) para que nosotros fuéramos liberados a costa de que nuestro compañero fuera condenado a una pena mayor (12 meses). Vemos, además, que la estrategia no cooperar (D) permite minimizar daños ante una posible traición de nuestro compañero.

\subsubsection{EQUILIBRIO DE NASH Y ÓPTIMOS EN SENTIDO SOCIAL Y DE PARETO. CONCEPTOS RELACIONADOS.}

Trataremos de entender la racionalidad del juego a partir de la información proporcionada por la matriz de pagos. Para ello, resulta de vital importancia la introducción de conceptos tan relevantes como el equilibrio de Nash, el óptimo de Pareto, el óptimo en sentido social o las estrategias dominantes.

11 Precisamente ésta ha sido la interpretación que hemos hecho de los pagos en nuestra investigación. 


\subsubsection{EQUILIBRIO DE NASH}

Un equilibrio de Nash es un conjunto de estrategias (una para cada uno de los n jugadores participantes en el juego) que tiene la propiedad de que la decisión de cada jugador es la mejor respuesta ante las elecciones de los otros n-1 jugadores (Holt \& Roth, 2004). Es decir, el Equilibrio de Nash describe una situación en la que ningún jugador tiene ningún incentivo individual para cambiar la estrategia que actualmente está siguiendo. $O$ lo que es lo mismo, ningún jugador puede obtener una recompensa mayor mediante un cambio de estrategia.

Retomando como ejemplo del dilema del prisionero, lo que cada prisionero desea es maximizar su propio beneficio (es decir, reducir su estancia en la cárcel). Sin embargo, el resultado final del juego no dependerá solamente de la decisión individual de cada prisionero, sino de la combinación las decisiones de ambos prisioneros. De entre todas las posibles estrategias, cada jugador escogerá aquella que maximice su beneficio teniendo en cuenta su expectativa sobre la decisión del otro jugador. Pues bien, decimos que una combinación de estrategias es un equilibrio de Nash si, aun cuando los jugadores anunciaran simultáneamente la estrategia que van a seguir, a ninguno de ellos le interesaría cambiar la estrategia que tenían pensado seguir antes de conocer la estrategia anunciada por el otro jugador.

Analicemos ahora los posibles equilibrios de Nash en el dilema del prisionero a partir de la matriz de pagos indicada en la Tabla 1.

Observemos que el par de estrategias (no cooperar, no cooperar) es el único equilibrio de Nash posible en el dilema del prisionero. Puede comprobarse, a partir de la matriz de pagos del juego, que cualquier otra combinación de estrategias haría que uno de los jugadores (o los dos) desearan haber escogido una estrategia distinta al conocer la estrategia que ha seguido el otro jugador. Sin embargo, cuando la combinación de estrategias constituye un equilibrio de Nash, aun cuando un jugador anunciara cuál va a ser su estrategia, el otro jugador no tendría incentivos para cambiar la estrategia que tenía pensado seguir. Puede comprobarse que esto no ocurre con el resto de pares de las estrategias de la matriz de pagos. 
Un juego, como veremos más adelante, puede tener varios equilibrios de Nash, aunque también puede ocurrir que no tenga ninguno ${ }^{12}$.

\subsubsection{ESTRATEGIA DOMINANTE}

Hablamos de estrategia dominante cuando ésta es la mejor opción sea cual sea la estrategia seguida por el otro jugador.

En el caso del dilema del prisionero, la estrategia dominante para ambos jugadores es no cooperar, ya que, tal como vemos en la matriz de pagos (Tabla 1), ésta es su mejor respuesta ante cualquier estrategia del oponente.

¿Cuál será la mejor opción para el jugador 1? Si éste supone que el jugador 2 va a cooperar (es decir, no va a confesar el crimen), entonces, según la matriz de pagos (primera columna), la mejor opción del jugador 1 sería no cooperar (es decir, confesar el crimen), ya que de esta manera el jugador 1 quedaría libre (0). Por otro lado, si el jugador 1 asume que el jugador 2 no va a cooperar (es decir, va a confesar el crimen), entonces según la matriz de pagos del juego (segunda columna), su mejor opción sería no cooperar (es decir, confesar el crimen), ya que así obtendría una pena de 3 meses frente a los 12 que obtendría si siguiera la otra estrategia.

Por tanto, independientemente de la estrategia que sigua el jugador 2, la mejor opción del jugador 1 es siempre no-cooperar. Es decir, la estrategia dominante del jugador 1 es no-cooperar ${ }^{13}$.

Cada juego tiene su propia matriz de pagos y no siempre existen estrategias dominantes.

12 Aunque es posible que un juego no tenga ningún equilibrio de Nash en estrategias simples, siempre tiene al menos un equilibrio de Nash en estrategias mixtas.

${ }^{13}$ La estrategia dominante del jugador 2 también es no-cooperar. El razonamiento es análogo al que hemos seguido con el jugador 1 . 


\subsubsection{3.ÓPTIMO DE PARETO}

Decimos que un par de estrategias (una por jugador) es óptimo en el sentido de Pareto si no hay otro par de estrategias en las que todos los jugadores reciban pagos iguales o mayores y al menos un jugador recibe un pago estrictamente mayor (Easley \& Kleinberg, 2010).

A partir de la matriz de pagos del juego del dilema del prisionero, observamos que los pares de estrategias (cooperar, cooperar), (cooperar, no-cooperar) y (no-cooperar, cooperar) son óptimos en el sentido de Pareto ya que, comparándolas una a una con los otros posibles pares de estrategias, siempre hay un jugador que recibe un pago estrictamente mayor (es decir, una condena estrictamente menor) y no existe otro par de estrategias en la que todos los jugadores reciben pagos iguales o mayores (es decir, condenas iguales o menores).

Sin embargo, el par de estrategias (no-cooperar, no-cooperar) no es Pareto-óptimo, ya que existe en el juego otra combinación de estrategias (cooperar-cooperar) cuyo resultado $(1,1)$ produce un pago mayor (condena menor) para ambos jugadores que la estrategia (no-cooperar, no-cooperar), la cual produce un pago menor (condena mayor): $(3,3)$.

Por lo tanto, hemos comprobado que en el juego del dilema del prisionero, el único par de estrategias que no es óptimo en el sentido de Pareto es precisamente el par de estrategias que constituye el único equilibrio de Nash del sistema.

\subsubsection{4.ÓPTIMO EN SENTIDO SOCIAL}

Decimos que un par de estrategias es un óptimo en sentido social si dicha estrategia maximiza la suma de los pagos de los jugadores.

A la vista de la matriz de pagos del juego, es fácil ver que desde el punto de vista social, el mejor resultado es aquel en el que los dos jugadores colaboran (es decir, ambos niegan el crimen), ya que éste es el caso en el que el beneficio conjunto es máximo (es decir, la suma de las dos penas es mínimo). 
Vemos que, aunque desde el punto de vista individual la mejor opción es no cooperar (equilibrio de Nash), la mejor opción desde el punto de vista social es cooperar (óptimo en sentido social). Consecuentemente, el hecho de que el sistema se encuentre en un equilibrio de Nash no conlleva necesariamente que se esté logrando el mejor resultado conjunto para los participantes, simplemente se está obteniendo el mejor resultado para cada uno de ellos considerados individualmente.

El dilema del prisionero no es un juego que simplemente sirva como ejemplo para ilustrar los conceptos básicos de la Teoría de Juegos, sino que su mecánica sirve para modelar la esencia de muchos dilemas sociales en los que la racionalidad individual choca con la racionalidad colectiva como, por ejemplo, el dilema de utilizar o no sustancias dopantes en una competición entre dos atletas (Easley \& Kleinberg, 2010): si un atleta toma sustancias dopantes y el otro no, el primero tendrá una ventaja sobre el segundo; si los dos las toman o ninguno de los dos las toman, ninguno tendrá más ventaja que el otro, sin embargo, el beneficio sería mayor si ninguno de los dos las tomasen (efectos secundarios, posibilidad de que sean descubiertos, etc.). Sin embargo, como ya hemos visto, el único equilibrio de Nash del sistema es (no cooperar, no cooperar), que en este ejemplo concreto se traduce en (doparse, doparse).

\subsubsection{OTROS CONCEPTOS DE TEORÍA DE JUEGOS UTILIZADOS}

\section{Estrategia evolutivamente estable:}

Se trata del primer concepto de equilibrio introducido en la Teoría de Juegos Evolutiva (Vega-Redondo, 2003) y fue propuesto por Maynard Smith y Price (1973). Se trata de un concepto de equilibrio tal que si la población adopta la estrategia que conduce a dicho equilibrio, el sistema es capaz de soportar la entrada de un número relativamente pequeño de agentes (mutantes) que juegan una estrategia distinta. Un estado evolutivamente estable es siempre un equilibrio de Nash, pero un equilibrio de Nash no tiene por qué ser necesariamente un estado evolutivamente estable (Young, 1998). 


\section{Equilibrio estocásticamente estable:}

Un equilibrio es estocásticamente estable si es robusto contra sacudidas aleatorias persistentes (y no sólo aisladas, como es asumido en el caso de los estados evolutivamente estables) (Young, 1998). Cuando un proceso evolutivo está sujeto a persistentes sacudidas estocásticas, el sistema permanece en algunos estados durante una proporción de tiempo más larga que en otros estados. Estos estados en los que el sistema se encuentra la mayor parte del tiempo reciben el nombre de estados estocásticamente estables.

\section{Estrategias mixtas:}

Decimos que un agente sigue una estrategia pura cuando la elección de dicha estrategia es determinista. En cambio, cuando puede elegir entre varias estrategias y escoge cada una de ellas con cierta probabilidad, hablamos de estrategias mixtas.

\section{Proceso ergódico:}

Se dice que un proceso modelado como una cadena de Markov homogénea, irreducible y aperiódica es ergódico. En este caso, el comportamiento medio a largo plazo es independiente del camino tomado por el sistema, y, por tanto, también lo es de las condiciones iniciales.

\section{Estado absorbente:}

Un estado es absorbente si, una vez el sistema ha alcanzado dicho estado, la probabilidad de que salga de él es nula. Cuando un sistema tiene un único estado absorbente, éste acabará en dicho estado absorbente con probabilidad 1, y lo hará independientemente de las condiciones iniciales. Por el contrario, cuando el sistema tiene varios estados absorbentes, éste alcanzará uno de sus estados absorbentes con probabilidad 1, pero la probabilidad de acabar en un estado absorbente en concreto dependerá de las condiciones iniciales (Izquierdo et al., 2009). 


\subsubsection{ANTECEDENTES DEL EQUILIBRIO DE NASH Y TRABAJOS POSTERIORES}

El concepto de equilibrio introducido por Nash fue presentado por primera vez en su artículo "The Bargaining Problem" (1950). Hasta la introducción del equilibrio de Nash, la Teoría de Juegos seguía los pasos de Neumann y Morgenstern (1944), quienes se limitaron a estudiar juegos de dos jugadores y suma-cero (es decir, juegos en los que las ganancias de un jugador se producían a costa de las pérdidas de otro jugador). Sin embargo, el equilibrio introducido por Nash era un concepto más general, sin restricciones en cuanto a la matriz de pagos ni el número de jugadores (Holt \& Roth, 2004).

Nash compartió el Premio Nobel de Economía en 1994 con John Harsanyi y Reinhard Selten. Harsanyi había extendido el equilibrio de Nash a una clase de juegos más amplia denominados juegos de información incompleta (juegos en los que no es necesario asumir que los jugadores conocen las preferencias y las elecciones factibles de los otros jugadores), mientras que Selten trabajó en refinamientos en el equilibrio de Nash. Él sostenía que los requisitos del equilibrio de Nash no eran condiciones suficientes en casos de racionalidad limitada (de hecho, Selten es reconocido en el campo de la racionalidad limitada y es considerado uno de los padres de la economía experimental).

Con el tiempo, la Teoría de Juegos comenzó a integrarse en la teoría económica, lo cual ayudó a la introducción de métodos experimentales en Economía. Prueba de la creciente interrelación entre la Economía y la Psicología (a través de métodos experimentales) es el premio Nobel de Economía de 2002, concedido a Daniel Kahneman (un psicólogo) y a Vernon Smith (un economista experimental).

La experimentación ha dado lugar a enfoques capaces de predecir cómo las personas se comportan en realidad cuando las hipótesis de racionalidad y conocimiento perfecto no se cumplen. La literatura experimental está llena de ejemplos tanto de juegos en los cuales el comportamiento observado converge rápidamente al comportamiento de equilibrio y de juegos en los que el equilibrio es un pobre indicador (Holt \& Roth, 2004). 


\subsubsection{TEORÍA DE JUEGOS Y ECONOMÍA}

Desde la formulación del Equilibrio de Nash, este concepto ha sido utilizado en numerosos ámbitos de la Ciencia, tales como la Sociología, la Biología, la Economía, etc. (Maynard-Smith, 1982; Vega-Redondo, 2003; Swedberg, 2001). Dado que esta tesis doctoral está enmarcada dentro del ámbito de la Organización de Empresas, nos centraremos en las aplicaciones económicas, pero sin dejar de lado el aspecto sociológico (racionalidad del ser humano).

El equilibrio de Nash sirve para explicar, por ejemplo, por qué las coordinaciones entre duopolios suelen fracasar, lo cual es, en realidad, una aplicación directa del dilema del prisionero. Supongamos que tenemos un duopolio con dos empresas con estructura de costes idénticas. Para aumentar el precio del producto en el mercado, ambas empresas acuerdan reducir la producción en la misma medida. Como consecuencia, la curva de demanda se mantiene inalterada, pero la curva de la oferta se desplaza hacia la izquierda, por lo que el precio del bien aumenta. El resultado es que ambas empresas aumentan sus beneficios en comparación con la situación inicial en la que no había acuerdo para reducir la producción.

Lo que ocurre es que las dos empresas, por separado, tienen un gran incentivo para traicionarse mutuamente. Si una de ellas aumenta su producción (y la otra no lo hace), la primera empresa venderá algunos de sus productos a compradores que potencialmente serían clientes de la segunda empresa (antes del acuerdo, las dos empresas tenían la mitad de la cuota de mercado). Como consecuencia, el beneficio de la primera empresa aumenta a costa de que la segunda reduzca su beneficio. Pero también puede ocurrir es que las dos aumenten su producción. En este caso, el beneficio que obtienen en el mismo que el de la situación anterior al acuerdo. Las posibles estrategias y resultados quedan recogidos en la matriz de pagos de la Tabla 2.

Empresa 2

Empresa 1

\begin{tabular}{|l|c|c|}
\cline { 2 - 3 } \multicolumn{1}{c|}{} & Cooperar & No cooperar \\
\hline Cooperar & 100,100 & $-30,250$ \\
\hline No cooperar & $250,-30$ & 0,0 \\
\hline
\end{tabular}

Tabla 2. Matriz de pagos que muestra los beneficios de las empresas que forman el duopolio en función de sus estrategias (cooperar o no cooperar). 
¿Cuál sería la estrategia más ventajosa que debería seguir la empresa 1 para aumentar sus beneficios? Al igual que en el caso del dilema del prisionero, la estrategia dominante es "no cooperar". Es decir, independientemente de la estrategia seguida por la empresa 2, para la empresa 1 siempre resulta más beneficioso elegir la estrategia "no cooperar". Análogamente, la estrategia "no cooperar" también es la más beneficiosa para la empresa 2. De ahí la tentación de ambas empresas para traicionarse mutuamente. Como consecuencia, es previsible que las dos empresas dejen de cooperar, situándose así en el único equilibrio de Nash del sistema (no cooperar, no cooperar).

\subsubsection{CLASIFICACIÓN DE LOS JUEGOS}

\begin{tabular}{|l|l|}
\hline $\begin{array}{l}\text { Simultáneos } \\
\text { vs. }\end{array}$ & $\begin{array}{l}\text { En un juego simultáneo, los jugadores exhiben sus decisiones } \\
\text { simultáneamente, mientras que en un juego secuencial, las } \\
\text { elecciones de los jugadores se llevan a cabo por turnos. } \\
\text { Como consecuencia, el segundo jugador tiene conocimiento } \\
\text { sobre la estrategia del primer jugador antes de tomar su } \\
\text { propia decisión. }\end{array}$ \\
\hline Información perfecta & $\begin{array}{l}\text { Un juego es de información perfecta si todos los jugadores } \\
\text { conocen las decisiones que han tomado los otros jugadores } \\
\text { con anterioridad. Como consecuencia, sólo los juegos } \\
\text { secuenciales pueden ser de información perfecta (los juegos } \\
\text { de información perfecta pueden considerarse un subconjunto } \\
\text { de los juegos secuenciales). }\end{array}$ \\
\hline $\begin{array}{l}\text { Información } \\
\text { completa }{ }^{14}\end{array}$ & $\begin{array}{l}\text { necesariamente la estrategia elegida por los jugadores a los } \\
\text { que se enfrentan. Sin embargo, sí conocen las posibles } \\
\text { estrategias y las posibles recompensas que pueden obtener. }\end{array}$ \\
\hline
\end{tabular}

${ }^{14}$ La disponibilidad de información suele venir acompañada de cierto orden: conocimiento común de información completa de orden cero significa que cada jugador tiene información completa. Si es de orden 1, significa que todos los jugadores saben que todos los jugadores tienen información completa. En general, el conocimiento común de orden n asume que el conocimiento común de orden n-1 es conocido por los jugadores. Si no se especifica ningún orden, se asume que el orden es infinito (esto produce un recursión infinita de asunciones compartidas) (Izquierdo, 2008). 


\begin{tabular}{|c|c|}
\hline $\begin{array}{l}\text { Simétricos } \\
\text { vs. } \\
\text { Asimétricos }\end{array}$ & $\begin{array}{l}\text { Un juego simétrico es aquel en el que el pago que se obtiene } \\
\text { al elegir una estrategia depende sólo de las estrategias } \\
\text { empleadas por los otros jugadores, pero no del jugador } \\
\text { concreto contra el que juegue. Es decir, decimos que un } \\
\text { juego es simétrico cuando las identidades de los jugadores } \\
\text { pueden cambiarse sin que esto afecte a la recompensa } \\
\text { obtenida para cada una de las posibles estrategias. }\end{array}$ \\
\hline Suma cero & $\begin{array}{l}\text { En un juego de suma cero, la suma de los pagos obtenidos } \\
\text { por los jugadores, para cada par de estrategias, es cero. En } \\
\text { otras palabras, en un juego de suma cero, para que un } \\
\text { jugador obtenga cierto beneficio es necesario que el otro } \\
\text { jugador pierda una cantidad equivalente al beneficio } \\
\text { obtenido por el primer jugador. }\end{array}$ \\
\hline $\begin{array}{l}\text { Cooperativos } \\
\text { vs. } \\
\text { no cooperativos }\end{array}$ & $\begin{array}{l}\text { En un juego cooperativo, los agentes no compiten entre sí, } \\
\text { sino que se esfuerzan por conseguir un objetivo común. Un } \\
\text { juego es no-cooperativo si es imposible que los jugadores se } \\
\text { comuniquen entre sí o colaboren de alguna manera. }\end{array}$ \\
\hline
\end{tabular}

Tabla 3. Clasificación de los juegos.

\subsubsection{RAMAS DE LA TEORÍA DE JUEGOS}

Hemos visto que conocer la matriz de pagos nos permite predecir el resultado del juego. Hemos considerado que la estrategia que escogían los agentes era aquella que maximizaba su beneficio, para lo cual hacían una predicción sobre la estrategia que iba a seguir su oponente. Sin embargo, existen una serie de cuestiones debemos plantearnos en torno a cómo toman decisiones las personas:

- ¿Se cumple, en la vida real, que los jugadores conozcan las posible estrategias de los otros jugadores?

- Y si esto es así, ¿cómo estima cada jugador la probabilidad con que sus oponentes se decidan por cada una de sus posibles estrategias?

- Si tenemos en cuenta la experiencia de situaciones similares en el pasado (memoria), ¿cómo utilizamos esta información para tomar decisiones? ¿Cuánta información de nuestra memoria empleamos en la práctica para la toma de decisiones? 
- En el supuesto de que los agentes hayan sido capaces de calcular las probabilidades con las que su oponente puede seguir cada una de sus posibles estrategias, ¿tendrán también la racionalidad suficiente como para tomar la decisión que maximice su beneficio?

- Suponiendo que tuvieran esta racionalidad, ¿vale la pena invertir tiempo y recursos para tomar la decisión?

- ¿Habría que considerar la exploración (decisiones al azar) para probar si conseguimos un mejor resultado con otras estrategias?

Hasta el momento, hemos considerado que los agentes tienen racionalidad ilimitada. Es decir, conocen las estrategias de sus oponentes, tienen toda la capacidad necesaria para estimar la estrategia más probable a seguir por sus oponentes y actúan en consecuencia, eligiendo la estrategia que mayor beneficio les reporta. Sin embargo, cuando se hacen asunciones sobre el significado de los pagos y sobre cómo se comportan (o deberían comportarse) los agentes, la Teoría de Juegos da lugar a tres ramas: Teoría de Juegos Clásica, Teoría de Juegos Evolutiva y Teoría de Aprendizaje en Juegos (Izquierdo, Izquierdo \& Vega-Redondo, 2012).

Mientras que la Teoría de Juegos Clásica asume que el ser humano es completamente racional, la Teoría de Juegos Evolutiva y la Teoría de Aprendizaje en Juegos adoptan una visión más realista según la cual las personas basan sus decisiones en una cantidad de datos limitada, usan modelos predictivos e incluso a veces tienen comportamientos poco racionales (Young, 1998).

La Teoría de Juegos Evolutiva es una rama de la Teoría de Juegos que estudia la evolución de grandes poblaciones de individuos que juegan repetidamente un juego y están expuestos a presiones evolutivas (selección, replicación y mutación) (Izquierdo, Izquierdo \& Vega-Redondo, 2012). Aquí, la estrategia que sigue cada uno de los agentes no viene dada por las decisiones que toman, sino por el grupo de individuos al que pertenecen. Cada uno de ellos tiene cierta adaptación (fitness) en función de si interactúa con otro individuo de su mismo grupo o de un grupo diferente. Los individuos que mejor se adaptan son los que tienen mayores posibilidades de reproducirse y, por tanto, los individuos de su clase tendrán mayores posibilidades de estar presentes en el futuro. La interpretación de la Teoría de Juegos Evolutiva aplicada a modelos económicos no es estrictamente biologicista, sino que puede ser interpretada, por ejemplo, como imitación de las estrategias más exitosas, etc. 
La Teoría de Aprendizaje en Juegos es la rama de la Teoría de Juegos que estudia las dinámicas de un grupo de individuos que juegan repetidamente un juego y que adaptan su estrategia a lo largo del tiempo como resultado de su experiencia. Asume que los jugadores aprenden a lo largo del tiempo sobre el juego y sobre el comportamiento de los demás.

\subsubsection{TEORÍA DE JUEGOS CLÁSICA (Classical Game Theory)}

Para la Teoría de Juegos Clásica, los individuos tienen capacidad de razonamiento y computación ilimitada para elegir la estrategia que maximiza su beneficio. La finalidad de la Teoría de Juegos Clásica es estudiar cómo deben comportarse estos agentes racionales para obtener el pago máximo en el juego. Dada la fortaleza de las hipótesis que se hacen en la Teoría de Juegos Clásica, no es sorprendente que la solución teórica que ésta proporciona muestre anomalías cuando la comparamos con lo observado en la realidad (Izquierdo, Izquierdo \& Vega-Redondo, 2012).

\subsubsection{TEORÍA DE JUEGOS EVOLUTIVA (Evolutionary Game Theory)}

Se considera que un modelo es evolutivo si en él existen los siguientes tres mecanismos: selección, replicación y mutación. La selección y la replicación son fuerzas que contribuyen a reducir la diversidad del sistema: los individuos mejor adaptados (los que tienen la mayor fitness) tienen mayor probabilidad de sobrevivir y así poder reproducirse. Es decir, tienen más probabilidades de que su especie esté presente en generaciones futuras, aumentando la proporción relativa de su especie frente a otras especies que se han adaptado peor (menor fitness). Por otro lado, existe otro mecanismo que consigue lo contrario: aumentar la diversidad del sistema: se trata del mecanismo de la mutación, que hace que surjan nuevas especies en el sistema, con una nueva fitness y, por tanto, con nuevas posibilidades en cuanto a posibilidad de reproducción y selección. 
El objetivo es identificar qué estrategias (es decir, qué especie) tiene más probabilidades de subsistir en el sistema y cuál será extinguida por las fuerzas selectivas. Estas cuestiones se pueden tratar matemáticamente a través de la llamada ecuación del replicador (replicator dynamics). Esta ecuación fue introducida por Taylor y Jonker (1978) y describe la evolución del número de individuos de cada especie teniendo en cuenta su influencia mutua en su fitness. Lo que hace es traducir a términos matemáticos los principios elementales de la selección natural: las estrategias (o los individuos que siguen cierta estrategia) que se reproducen más eficientemente se esparcen, desplazando a aquellos con menor fitness (Roca, Cuesta \& Sánchez, 2009).

Matemáticamente, la ecuación del replicador nos dice que la variación en el número medio de individuos de una especie i $\left(\dot{x}_{\mathrm{i}}\right)$ es proporcional al número de individuos de esa especie presentes en la actualidad $\left(x_{\mathrm{i}}\right)$ y a la diferencia entre su fitness $\left(\left(W_{x}\right)_{i}\right)$ y el fitness medio $\left(x^{T} W_{x}\right)$.

$$
\dot{x}_{\mathrm{i}}=x_{\mathrm{i}}\left[\left(W_{x}\right)_{i}-x^{T} W_{x}\right]
$$

La ecuación del replicador supone una serie de asunciones. En primer lugar, la población debe ser infinita. En segundo lugar, la población debe estar bien mezclada y sin mutaciones. Por población bien mezclada, nos referimos a que todos los individuos tengan la misma probabilidad de interactuar con cualquier otro individuo de la población. El modelado basado en agentes ha permitido relajar estas hipótesis manteniendo el marco evolutivo en muchos modelos (Izquierdo, 2008).

\subsubsection{TEORÍA DE APRENDIZAJE EN JUEGOS (Learning Game Theory)}

En la Teoría de Juegos Clásica se consideraba que los jugadores eran perfectamente racionales, de tal forma que siempre eran capaces de elegir la estrategia maximizadora de su beneficio. Por otro lado, en la Teoría de Juegos Evolutiva, se consideraba que los individuos no eligen ninguna estrategia, sino que cada especie tiene una estrategia que le viene dada por la especie a la que pertenece. Por su parte, en la Teoría de Aprendizaje en Juegos, la estrategia de los agentes varía a lo largo del desarrollo del juego, a medida que los agentes van aprendiendo sobre el juego y sobre las estrategias empleadas por los 
otros agentes. En base a este aprendizaje, los jugadores pueden cambiar de estrategia con la esperanza de obtener mejores resultados en juegos futuros.

El cambio de estrategia puede hacerse, bien imitando el comportamiento de otro agente, bien actualizando nuestras expectativas sobre la estrategia jugada por el resto de los jugadores. Según la manera en la que los agentes cambian su estrategia, existen varios modelos de aprendizaje (Izquierdo, Izquierdo \& Vega-Redondo, 2012; Vega-Redondo, 2003; Fudenberg \& Levine, 1998; Young, 2004): Reinforcement learning (las acciones que han sido exitosas en el pasado tienden a repetirse en el futuro, mientras que las elecciones que dieron lugar a experiencias insatisfactorias son evitadas); learning by imitation (un jugador adopta la estrategia de otro jugador); static perceptions and myopic response (los agentes asumen que los demás agentes mantienen inalterada su decisión actual y seleccionan la estrategia que maximiza su pago); fictitious play (el agente asume que cada uno de sus oponentes está jugando cierta estrategia mixta, y su estimación de esta estrategia mixta es igual a la frecuencia con que el oponente ha elegido cada una de las posibles acciones hasta el momento).

Existen otros modelos de aprendizaje que no asumen que los agentes responden de manera óptima, sino que pretenden describir cómo se comportaría realmente un humano (es decir, de una manera que no es totalmente racional). Muchos de estos modelos de aprendizaje son recogidos bajo el nombre de Behavioural Game Theory.

Nuestro trabajo de investigación toma ideas de las tres ramas de la Teoría de Juegos que acabamos de describir. Nuestro modelo de partida es el Modelo de Clases de Axtell, Epstein y Young (2000), en el que los agentes van actualizando sus expectativas sobre las decisiones del resto de los jugadores a lo largo del tiempo, lo cual es claramente una idea tomada de la Teoría de Aprendizaje en Juegos. Sin embargo, no debemos olvidar que el juego de la demanda de Nash original (Nash, 1953) fue concebido como un juego de un único movimiento (one-shot game) en la que los agentes no tenían ocasión de aprender, pero que contaban con racionalidad ilimitada, lo cual encaja con lo que posteriormente se llamó Teoría de Juegos Clásica. En cuanto a la Teoría de Juegos Evolutiva, se han tomado algunos conceptos procedentes de esta rama de la Teoría de Juegos, como por ejemplo el de estrategia evolutivamente estable. 


\subsection{REDES COMPLEJAS}

\subsubsection{INTRODUCCIÓN}

Una expresión utilizada con frecuencia al descubrir que una persona determinada tiene cierta relación con nosotros a través de nuestros vínculos sociales es: "iqué pequeño es el mundo!". Lo que ocurre en realidad es que todas las personas estamos conectadas de tal forma que es posible relacionar dos personas cualesquiera a través de un número relativamente pequeño de saltos. Esto es lo que se conoce, como efecto de pequeñomundo.

Una de las primeras personas en observar el efecto de pequeño-mundo fue Frigyes Karinthy. Karinthy no se dedicaba a la investigación científica - era un escritor -, pero en su obra Chains (1930), narraba cómo uno de sus personajes de ficción era capaz de conectar cualquier persona en el mundo a través de un número no muy grande de saltos (Barabási, 2002).

Observamos, por tanto, que los indicios de que la sociedad en la que vivimos presenta la propiedad de pequeño-mundo han existido desde hace varias décadas. Erdős y Rényi proponían en 1959 su Modelo de Red Aleatoria. Las redes generadas por dicho modelo cumplían con la propiedad de pequeño-mundo. Erdős y Rényi demostraron que, en una red en la que se tienen enlaces entre pares de nodos generados de forma aleatoria (ver sección 2.3.5.1), basta con que el número medio de enlaces por nodo sea igual a 1 para que la emerja un clúster del que forman parte la mayor parte de los nodos de la red. En este clúster, no sólo los nodos están interconectados, sino que además lo están a través de un número pequeño de saltos. Por consiguiente, el sencillo modelo de Erdős y Rényi presenta la propiedad de pequeño-mundo. Sin embargo, como veremos en la sección 2.3.5.1, este modelo no presenta la propiedad de alto clustering que también está presente en las redes sociales.

El origen de la investigación científica en redes de pequeño-mundo les es atribuida a Milgram (1967) y a Pool y Kochen (1978). 
En 1967, el sociólogo Stanley Milgram llevó a cabo un experimento consistente en elegir un destinatario (se escogió a una persona que vivía en la Coste Este de EEUU) y proponer a ciertas personas que vivían en la zona central de EEUU (y que desconocían al destinatario) que le enviaran una carta. Los datos de los que disponían los emisores de la carta eran tan solo el nombre, la profesión y el área geográfica en la que vivía el destinatario. Por ello, lo que debían hacer era enviar la carta a un intermediario que creyera que tenía posibilidades de conocer al destinatario. A su vez, los individuos intermedios que iban recibiendo la carta tenían que reenviarla a otra persona que pensara que tenía posibilidades de conocer al destinatario. El resultado del experimento fue que las cartas que alcanzaron su destino lo hicieron, en promedio, a través de cinco intermediarios, lo cual equivale a decir que el origen y el destino de la carta estaban separados, en promedio, a través de seis conexiones en la red. Esta fue una de las primeras demostraciones de que los seres humanos estamos integrados en una red social en la que existe un "camino corto" que permite atravesarla de un extremo a otro. En otras palabras, nuestra sociedad posee la propiedad de pequeño-mundo. El experimento de Milgram fue el origen de la expresión que saltó a la fama como "Seis Grados de Separación". Sin embargo, a pesar de que la expresión está basada precisamente en los seis saltos que, en promedio, debían hacer las cartas en este experimento, Milgram nunca utilizó esta expresión. La expresión comenzó a propagarse cuando el dramaturgo John Guare, en 1990 diera este nombre a una de sus obras.

A pesar de la relevancia de la propiedad de pequeño-mundo que presenta nuestra sociedad, ésta no es la única propiedad que la caracteriza. Nuestra sociedad, presenta, además, una propiedad conocida como alto clustering. Esta propiedad se traduce en que, en nuestra sociedad, existe una alta probabilidad de que un amigo de nuestro amigo sea a la vez amigo nuestro. En otras palabras, nuestra sociedad presenta la peculiaridad de que existe una alta probabilidad de que las personas tengamos amigos en común.

La Comunidad Científica ha estado trabajando durante los últimos años en la búsqueda de modelos teóricos de redes que presenten propiedades similares a las que se observan en las redes observadas en la vida real. Concretamente, es sabido que las redes sociales presentan ciertas propiedades, como son alto clustering, efecto de pequeño-mundo, una distribución de grado que sigue una ley de potencias (lo cual implica la presencia de nodos con un número excepcionalmente alto de enlaces - hubs) y asortatividad positiva (Vega-Redondo, 2007). 
Watts y Strogatz (1998) propusieron un modelo teórico de red que presentaba simultáneamente las propiedades de alto clustering y efecto de pequeño-mundo. A través de un algoritmo de red sencillo (ver sección 2.3.5.3) obtuvieron una familia de redes que interpolaban entre el orden y la aleatoriedad. En la transición, hallaron una clase de redes (las llamadas redes de pequeño-mundo) que presentaban simultáneamente un alto clustering y el efecto de pequeño-mundo, dos de las propiedades que presentan las redes sociales reales.

Posteriormente, Barabási y Albert (1999) presentaron un modelo de red que se caracteriza por la existencia de hubs (nodos con un número de enlaces mucho más elevado que el resto de los nodos de la red, ver sección 2.3.5.4). Esta propiedad no sólo está presente en las redes sociales sino también en otros tipo de redes como internet, la Web o redes de colaboración (Newman, 2003).

Dado que en esta tesis doctoral queremos analizar la emergencia y difusión de normas a través de redes sociales, basaremos nuestro estudio en las redes de Watts y Strogatz, puesto que estas redes pueden presentar simultáneamente alto clustering y el efecto de pequeño-mundo, propiedades que están presentes en las redes sociales reales. No obstante, el estudio de las redes abarca otros campos muy diversos, desde las Matemáticas (grafos), Tecnología (redes eléctricas, redes de transporte), Comunicación (la Web, planificación de la jerarquía de comunicaciones dentro de una empresa), Medicina (difusión de enfermedades contagiosas), Biología (cadenas tróficas, relaciones entre seres vivos), etc. (Solé, 2009).

Existen en la literatura numerosos modelos en los que se asume que la relación entre los agentes que componen el sistema es aleatoria, sin tener en cuenta el hecho de que los seres humanos formamos parte de una red social y que este hecho influye en nuestra toma de decisiones. En esta tesis doctoral hemos aplicado esta importante propiedad de la condición humana a un modelo económico bien conocido: el Modelo de Clases (Axtell, Epstein \& Young, 2000).

A continuación mostraremos algunos conceptos relacionados con redes a los que hemos hecho referencia de manera habitual en los trabajos de investigación. 


\subsubsection{ELEMENTOS DE UNA RED}

En términos generales, una red es un conjunto de elementos llamados vértices (o nodos) con conexiones entre ellos (llamadas enlaces ${ }^{15}$ ) (Newman, 2003). No obstante, este término tiene distintos significados en distintas disciplinas. Concretamente, en las Ciencias Sociales, una red suele definirse como un conjunto de actores (agentes) que pueden tener una relación entre ellos (enlaces) (Izquierdo \& Hanneman, 2006). Un ejemplo de red puede visualizarse en la Figura 7.

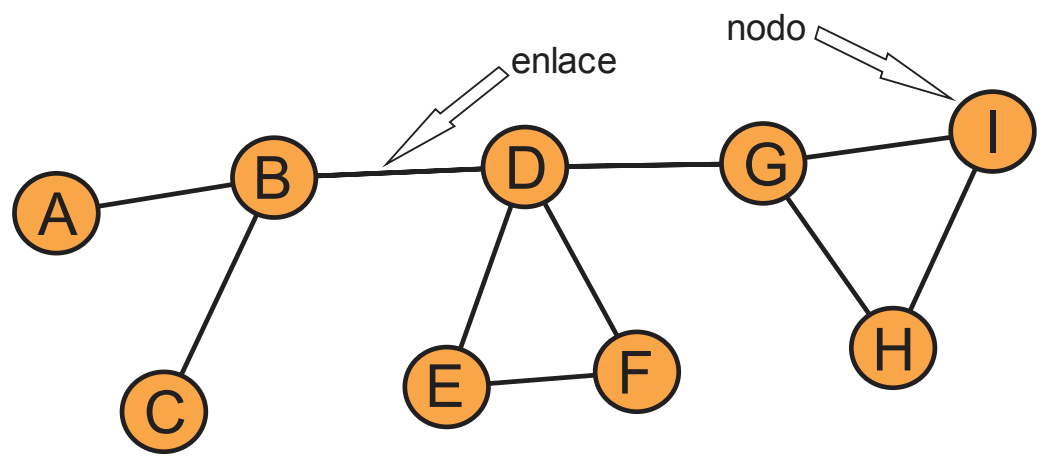

Figura 7. Una red con 9 nodos y 10 enlaces.

Un enlace entre dos nodos puede representar distintos tipos de relaciones: si la relación que representa el enlace es bidireccional (ej. ser hermano de), el enlace se suele representar con un segmento que une ambos nodos; en cambio, si la relación representa el origen y el objetivo entre enlaces (ej. ser padre de), el enlace suele representarse a través de una flecha. En este caso concreto, los enlaces se suelen llamar arcos.

${ }^{15}$ En inglés: links, edges o ties. 


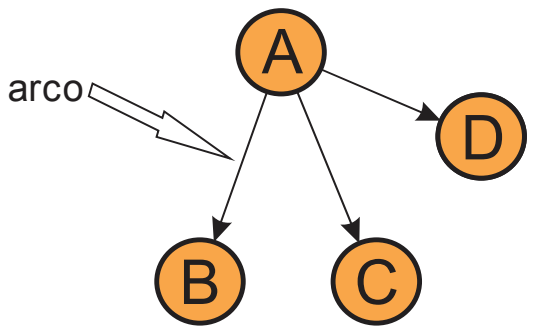

Figura 8. Red dirigida.

Aquellas redes en las que todos los enlaces son arcos, reciben el nombre de redes dirigidas (directed networks), mientras que aquellas redes en las que no existe ningún arco reciben el nombre de redes no-dirigidas (undirected networks). Por otro lado, si algunos de los enlaces son arcos y otros no, hablamos de redes mixtas (mixed networks). La Figura 7 muestra una red no-dirigida, mientras que la Figura 8 muestra una red dirigida.

\subsubsection{PROPIEDADES BÁSICAS DE UNA RED}

Con el objetivo de facilitar la comprensión de los trabajos de investigación que se incluyen en el bloque 2, llevaremos a cabo una introducción a algunos conceptos relacionados con redes a los que haremos referencia frecuentemente en dichos artículos. En la presente sección mostraremos algunas propiedades básicas de una red. En la sección 2.3.4 procederemos a definir una serie de conceptos relacionados con redes.

\subsubsection{DENSIDAD DE LA RED}

La densidad de una red se define como el cociente entre el número de enlaces de la red y el número máximo de enlaces que ésta podría tener.

Sea $\mathrm{m}$ es el número de enlaces de la red y n el número de nodos. Si la red es no-dirigida, el número máximo de enlaces será: $\mathrm{n} \cdot(\mathrm{n}-1) / 2$; si la red es dirigida el número máximo de enlaces es: $n \cdot(n-1)$. Por lo tanto, en función del tipo de red, la densidad de la misma vendrá dada por una de las siguientes expresiones: 
Para redes no-dirigidas: densidad $=\frac{\mathrm{m}}{n(n-1) / 2}$

Para redes dirigidas: $\quad$ densidad $=\frac{\mathrm{m}}{n(n-1)}$

En el caso de la red de la Figura 7 (red no-dirigida), la densidad es $10 /(9 \cdot 8) / 2=$ 0.277. En el caso de la red de la Figura 8 (red dirigida), la densidad es $3 /(4 \cdot 3)=$ 0.25 .

\subsubsection{GRADO DE UN NODO. GRADO MEDIO DE UNA RED. DISTRIBUCIÓN DE GRADO DE UNA RED.}

El grado (o degree) de un nodo es el número de enlaces que tiene dicho nodo. En caso de que la red sea dirigida hablamos de in-degree (número de enlaces incidentes en el nodo) y de out-degree (número de enlaces salientes del nodo). Si la red es no-dirigida hablaremos simplemente de grado (degree).

El grado medio de una red no es más que el promedio del grado de todos los nodos de la red.

La Tabla 4 y la Tabla 5 indican el grado de cada uno de los nodos de la Figura 7 y la Figura 8, respectivamente. Se indica también el grado medio de cada una de las redes.

\begin{tabular}{|c|c|}
\hline \multicolumn{2}{|c|}{ Red de la Figura 7} \\
\hline Nodo & Grado \\
\hline A & 1 \\
\hline B & 3 \\
\hline C & 1 \\
\hline D & 4 \\
\hline E & 2 \\
\hline F & 2 \\
\hline G & 3 \\
\hline H & 2 \\
\hline I & 2 \\
\hline Grado medio: $20 / 9=2.222$ \\
\hline
\end{tabular}

Tabla 4. Grado de cada uno de los nodos de la red no-dirigida de la Figura 7. Grado medio de la red. 


\begin{tabular}{|c|c|c|}
\hline \multicolumn{3}{|c|}{ Red de la Figura 8} \\
\hline Nodo & In-degree & Out-degree \\
\hline $\mathrm{A}$ & 0 & 3 \\
\hline $\mathrm{B}$ & 1 & 0 \\
\hline $\mathrm{C}$ & 1 & 0 \\
\hline $\mathrm{D}$ & 1 & 0 \\
\hline & In-degree medio: $3 / 4=0.75$ & Out-degree medio: $3 / 4=0.75$ \\
\hline
\end{tabular}

Tabla 5. Grado de cada uno de los nodos de la red dirigida de la Figura 8. In-degree medio y outdegree medio.

Sea $p_{k}$ la fracción de los nodos de una red que tienen grado $k$ (lo cual equivale a decir que $k$ es la probabilidad de que un nodo elegido uniformemente al azar tenga grado $k$ ). Si representamos $p_{k}$ en función de $k$ obtenemos un histograma que se conoce con el nombre de distribución de grado de una red. En la sección 2.3.5 se muestra la distribución de grado de varios tipos de redes: red aleatoria (Figura 14), red regular (Figura 16) y red de Barabási-Albert (Figura 21).

\subsubsection{DISTANCIA ENTRE AGENTES (distancia geodésica)}

La distancia geodésica entre dos nodos es el número mínimo de enlaces que hay que atravesar para ir desde un nodo hasta el otro.

Por ejemplo, los nodos A e I en la Figura 7 están separados por una distancia de 4.

\subsubsection{EXCENTRICIDAD}

Para conocer la excentricidad de un nodo, calculamos la distancia geodésica entre ese nodo y todos los demás, y nos quedamos con la mayor de ellas. En la Tabla 6 se indican las excentricidades de cada uno de los nodos de la Figura 7: 


\begin{tabular}{|c|c|}
\hline \multicolumn{2}{|c|}{ Red de la Figura 7} \\
\hline Nodo & Excentricidad \\
\hline A & 4 \\
\hline B & 3 \\
\hline C & 4 \\
\hline D & 2 \\
\hline E & 3 \\
\hline F & 3 \\
\hline G & 3 \\
\hline H & 4 \\
\hline I & 4 \\
\hline
\end{tabular}

Tabla 6. Excentricidad de los nodos de la red de la Figura 7.

Los nodos con mayor excentricidad reciben el nombre de nodos periféricos; mientras que los nodos con menos excentricidad constituyen el centro de la red. A raíz de los datos de la Tabla 6, podemos decir que, en la Figura 7, el nodo D constituye el centro de la red, y los nodos A, C, H e I son los nodos periféricos.

\subsubsection{DIÁMETRO DE LA RED}

Se trata de la mayor excentricidad de entre todos los nodos de la red. A partir de los datos de la Tabla 6, podemos afirmar que el diámetro de la red de la Figura 7 es 4.

\subsubsection{RADIO DE LA RED}

Es la mínima excentricidad de entre todos los nodos de la red. Los datos de la Tabla 6 nos indican que el radio de la red de la Figura 7 es 2.

\subsubsection{CENTRALIDAD DE UN NODO}

La centralidad de un nodo es una medida que nos permite conocer cuáles son los nodos mejor conectados en una red. En otras palabras, la centralidad nos permite conocer cuáles son los nodos más influyentes dentro de una red (Newman, 2003). 
Existen varias unidades para medir la centralidad en función del criterio empleado. A continuación describiremos las tres más habituales:

- Centralidad de $\operatorname{grado}^{16}$ : Se dice que un nodo es central si éste presenta alto grado.

- Centralidad de proximidad ${ }^{17}$ : Un nodo se considera central si es fácilmente accesible (es decir, si se encuentra próximo a todos los demás nodos).

- Centralidad de betweenness ${ }^{18}$ : Un nodo es considerado central si se encuentra sobre un elevado número de caminos geodésicos entre nodos.

\subsection{CENTRALIDAD DE GRADO (DEGREE CENTRALITY)}

La centralidad de grado de un nodo $c_{D}(x)$ es, simplemente el número de enlaces que tiene dicho nodo.

$$
c_{D}(x)=\text { grado del nodo } x
$$

La expresión anterior es absoluta en el sentido de que sólo nos permite comparar la centralidad de nodos dentro de una misma red. Para poder comparar la centralidad de grado entre nodos de distintas redes, empleamos la centralidad de grado relativa, $C_{D}(x)$, que se obtiene dividiendo la centralidad absoluta entre el número máximo de enlaces que puede tener un nodo (que es $n$ - 1 en redes no-dirigidas y sin bucles):

$$
C_{D}(x)=\frac{c_{D}(x)}{n-1}
$$

La Tabla 7 muestra la centralidad de grado (relativa y absoluta) de cada uno de los nodos de la red de la Figura 7.

\footnotetext{
16 Degree centrality

17 Closeness centrality

18 Betweenness centrality
} 


\begin{tabular}{|c|c|c|}
\hline \multicolumn{3}{|c|}{ Red de la Figura 7 } \\
\hline Nodo & $\begin{array}{c}\text { Centralidad de grado } \\
\text { absoluta }\end{array}$ & $\begin{array}{c}\text { Centralidad de grado } \\
\text { relativa }\end{array}$ \\
\hline A & 1 & 0.11111111 \\
\hline B & 3 & 0.33333333 \\
\hline C & 1 & 0.11111111 \\
\hline D & 4 & 0.44444444 \\
\hline E & 2 & 0.22222222 \\
\hline F & 2 & 0.22222222 \\
\hline G & 3 & 0.33333333 \\
\hline H & 2 & 0.22222222 \\
\hline I & 2 & 0.22222222 \\
\hline
\end{tabular}

Tabla 7. Centralidad de grado de los nodos de la red de la Figura 7. Se ha resaltado en color rojo el nodo central según esta definición de centralidad.

\subsection{CENTRALIDAD DE PROXIMIDAD (CLOSENESS CENTRALITY)}

La centralidad de proximidad de un nodo $c_{C}(x)$ viene dada por la siguiente expresión:

$$
c_{C}(x)=\frac{1}{\sum_{y \in U} d(x, y)}
$$

Donde $d(x, y)$ es la distancia geodésica entre los nodos $x$ e $y, \mathrm{y}$ U es el conjunto de todos los nodos de la red.

En la expresión anterior, sólo se tienen en cuenta los nodos alcanzables desde el nodo $x$.

Según esta definición de centralidad, el nodo más central puede interactuar rápidamente con todos los otros nodos debido a su proximidad.

Esta medida de centralidad es preferible a la centralidad de grado, ya que no tiene en cuenta solamente las condiciones directas entre nodos, sino también las conexiones indirectas. 
Al igual que en el caso de la centralidad de grado, para comparar la centralidad de nodos de redes distintas necesitamos definir una centralidad relativa, $C_{C}(x)$. En el caso de la centralidad de proximidad, la expresión es la siguiente:

$$
C_{C}(x)=(n-1) \cdot c_{C}(x)
$$

La Tabla 8 muestra la centralidad de proximidad (relativa y absoluta) de cada uno de los nodos de la red de la Figura 7.

\begin{tabular}{|c|c|c|}
\hline \multicolumn{3}{|c|}{ Red de la Figura 7 } \\
\hline Nodo & $\begin{array}{c}\text { Centralidad de } \\
\text { proximidad absoluta }\end{array}$ & $\begin{array}{c}\text { Centralidad de proximidad } \\
\text { relativa }\end{array}$ \\
\hline A & 0.04545455 & 0.3636364 \\
\hline B & 0.06666667 & 0.53333333 \\
\hline C & 0.04545455 & 0.36363636 \\
\hline D & 0.08333333 & 0.66666667 \\
\hline E & 0.05555556 & 0.44444444 \\
\hline F & 0.05555556 & 0.44444444 \\
\hline G & 0.06666667 & 0.53333333 \\
\hline H & 0.04761905 & 0.38095238 \\
\hline I & 0.04761905 & 0.3809524 \\
\hline
\end{tabular}

Tabla 8. Centralidad de proximidad de los nodos de la red de la Figura 7. Se ha resaltado en color rojo el nodo central según esta definición de centralidad.

\subsection{CENTRALIDAD DE BETWEENNESS (BETWEENNESS CENTRALITY)}

La betweenness de un nodo $c_{B}(x)$ es el número de caminos más cortos que pasan a través de dicho nodo. Para calcularla, se computa, para cada par de nodos de la red, la fracción de todos los caminos más cortos que pasan a través de dicho nodo.

$$
c_{B}(x)=\sum_{y<z} \frac{\text { número de caminos más cortos entre y y } z \text { a través del nodo } x}{\text { número de caminos más cortos entre y y } z}
$$

La betweenness nos da una idea de la importancia del nodo para detener el flujo de información a través de la red. 
La medida de la centralidad de betweenness relativa para una red no-dirigida, $C_{B}(x)$, viene dada por:

$$
C_{B}(x)=\frac{c_{B}(x)}{(n-1)(n-2) / 2}
$$

La Tabla 9 muestra la centralidad de betweenness (relativa y absoluta) de cada uno de los nodos de la red de la Figura 7.

\begin{tabular}{|c|c|c|}
\hline \multicolumn{3}{|c|}{ Red de la Figura 7 } \\
\hline Nodo & $\begin{array}{c}\text { Centralidad de } \\
\text { betweenness absoluta }\end{array}$ & $\begin{array}{c}\text { Centralidad de } \\
\text { betweenness relativa }\end{array}$ \\
\hline A & 0.00000000 & 0.00000000 \\
\hline B & 13.00000000 & 0.46428571 \\
\hline C & 0.00000000 & 0.00000000 \\
\hline D & 21.00000000 & 0.75000000 \\
\hline E & 0.00000000 & 0.00000000 \\
\hline F & 0.00000000 & 0.00000000 \\
\hline G & 12.00000000 & 0.42857143 \\
\hline H & 0.00000000 & 0.00000000 \\
\hline I & 0.00000000 & 0.00000000 \\
\hline
\end{tabular}

Tabla 9. Centralidad de betweenness de los nodos de la red de la Figura 7. Se ha resaltado en color rojo el nodo central según esta definición de centralidad.

Observamos que, en el caso de la red de la Figura 7, las tres medidas de centralidad coinciden en que el nodo D es en nodo central. Sin embargo, esto no ocurre siempre así. De hecho, distintas medidas de centralidad pueden proporcionar resultados completamente diferentes. Por esta razón, la medida de centralidad empleada debe elegirse cuidadosamente.

Por ejemplo, en la red que se muestra en la Figura 9, los nodos 33, 53, 32 y 48 (señalados en color rojo) son los nodos más centrales según la definición de centralidad de betweenness, ya que a través de estos nodos pasan la mayoría de los caminos más cortos entre nodos. Sin embargo, el nodo 61 (señalado en morado) es el nodo más central según la definición de centralidad de grado, ya que es el nodo que tiene más enlaces. 


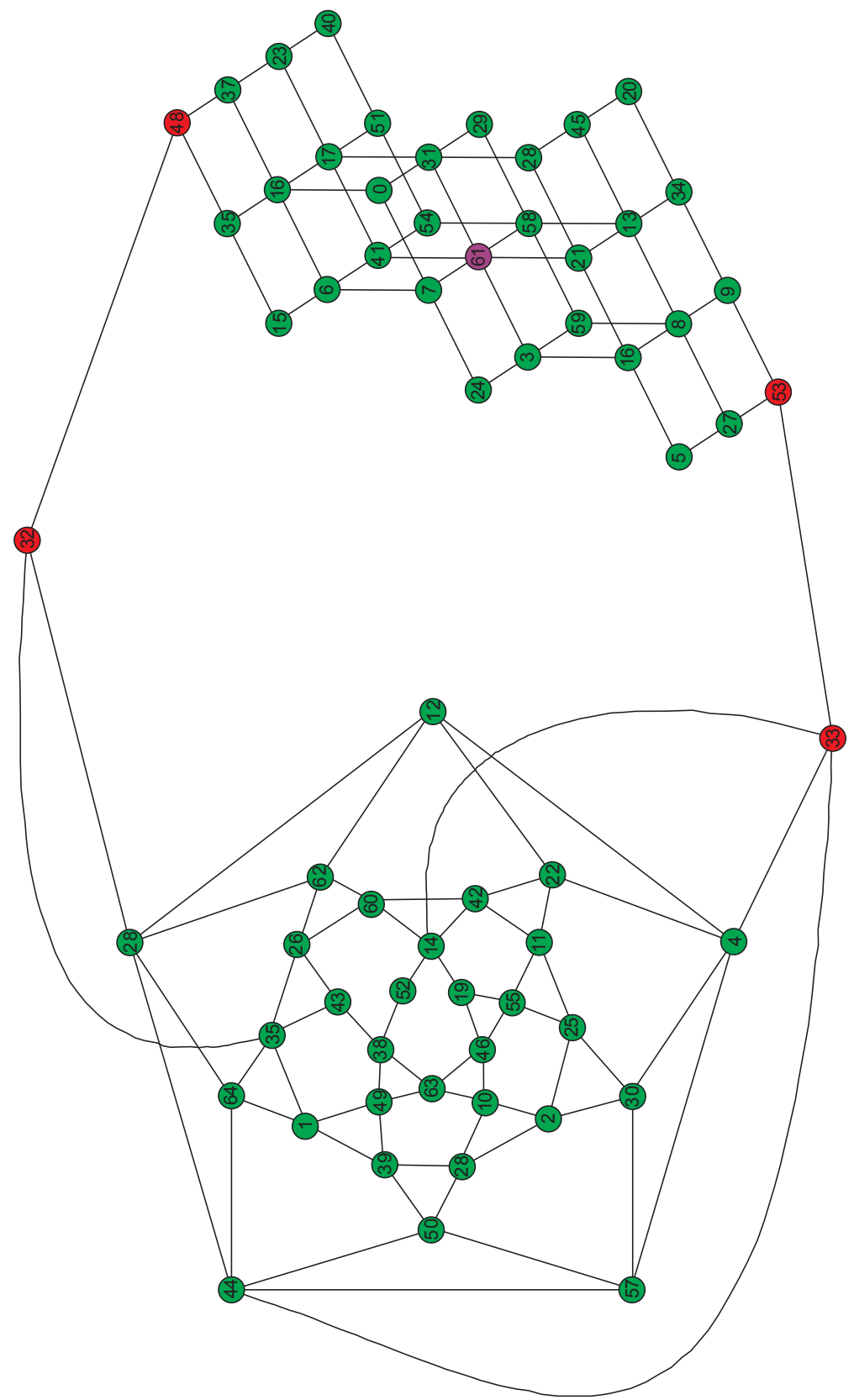

Figura 9. Ejemplo de red en el que las distintas definiciones de centralidad dan lugar a distintos nodos centrales. En rojo, los nodos más centrales según la definición de centralidad de betweenness. En morado, el nodo más central según la definición de centralidad de grado. Adaptado de (Mrvar, s.f.). 


\subsubsection{CONCEPTOS RELACIONADOS}

\subsubsection{CLUSTERING}

En las redes de alto clustering, si el nodo A está conectado al nodo B, y en el nodo B está conectado al nodo $\mathrm{C}$, entonces existe una alta probabilidad de que al nodo $\mathrm{C}$ esté conectado con el nodo A. En otras palabras, en términos de redes sociales, una red presenta alto clustering si existe una alta probabilidad de que los amigos de nuestros amigos sean a la vez nuestros amigos (Newman, 2003).

Más formalmente, el clustering es la probabilidad media de que dos vértices que son vecinos tengan como vecino a otro vértice y ellos dos sean vecinos entre sí.

En el interior de los nodos de cada una de las cuatro redes de la Figura 10 se muestra el coeficiente de clustering de cada nodo $\left(\mathrm{C}_{\mathrm{i}}\right)$. Para calcularlo, se ha dividido el número de enlaces existentes entre los vecinos de cada nodo entre el número máximo de enlaces que podrían existir entre sus vecinos ${ }^{19}$.

Debe tenerse en cuenta que, para nodos con grado 0 (esto es, nodos aislados), tanto el numerador como el denominador se hacen cero. En este caso tomamos $C_{i}=0$.

El coeficiente de clustering de un nodo A es 1 si todos los vecinos de A también están conectados entre sí, y 0 si ninguno de los vecinos de A tiene ninguna conexión entre sí. Para nodos con menos de dos vecinos, el coeficiente de clustering no está definido.

${ }^{19}$ Si un nodo tiene $\mathrm{n}$ vecinos, el número máximo de enlaces que pueden existir en una red nodirigida es $n \cdot(n-1) / 2$. 

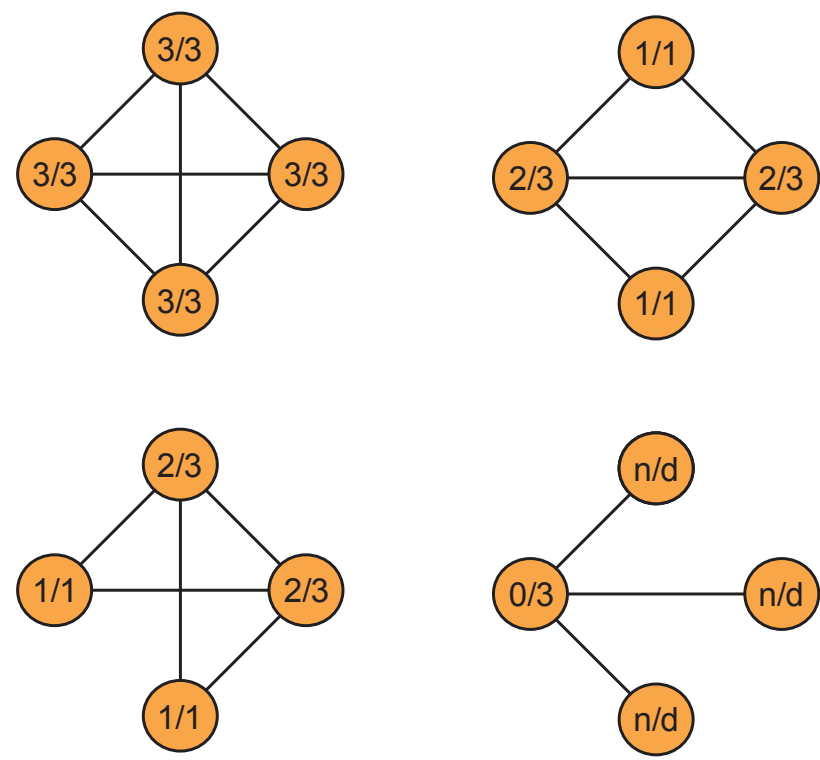

Figura 10. Coeficiente de clustering de varias redes no-dirigidas. Adaptado de (Izquierdo \& Hanneman, 2006).

Una vez calculado el coeficiente de clustering de cada uno de los nodos $\left(\mathrm{C}_{\mathrm{i}}\right)$, podemos calcular el coeficiente de clustering de la red (C) aplicando la siguiente expresión (promedio del coeficiente de clustering de cada uno de los nodos de la red):

$$
C=\frac{1}{n} \sum_{i}^{\mathrm{n}} C_{i}
$$

Aplicando la ecuación anterior, los coeficientes de clustering resultantes de las cuatro redes de la Figura 10 son, respectivamente, 1, 5/6, 5/6, 0.

\subsubsection{COMPONENTE}

Es el nombre que recibe un grupo conexo de nodos. La Figura 11 muestra una red con dos componentes. Los nodos pertenecientes a un mismo componente se muestran con un mismo color. Como puede observarse, por definición, no existe ningún enlace que unan nodos pertenecientes a distintos componentes. 


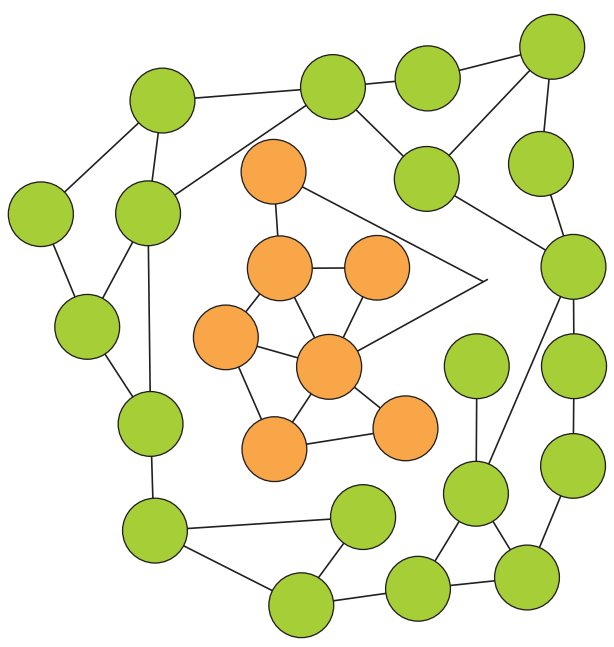

Figura 11. Red con dos componentes.

\subsubsection{ESTRUCTURA DE COMUNIDAD}

Una comunidad es un subconjunto de nodos que se encuentran conectados entre sí de una manera relativamente densa, pero escasamente conectado a otros grupos de nodos densamente conectados entre sí. (Porter, Onnela \& Mucha, 2009). La Figura 12 muestra el caso idealizado de estructura de comunidad. Cada una de las comunidades se encuentra identificada por un color.

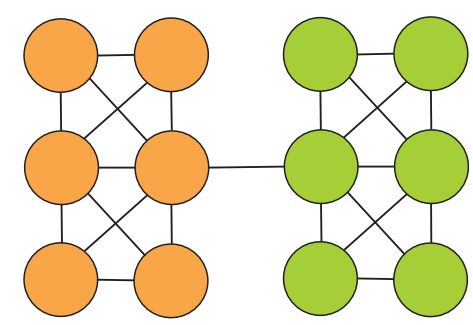

Figura 12. Red con estructura de comunidad.

Este tipo de estructura local puede ser identificada fácilmente en una amplia variedad de contextos sociales: familias, amigos, grupos virtuales en internet, vecindarios, etc. Existe en la literatura una amplia variedad de ejemplos de redes que presentan estructura de 
comunidad: redes de colaboración científica (Girvan \& Newman, 2002), redes de interacciones de email entre empleados de universidad (Guimerà et al., 2006), etc.

El proceso de detección de comunidades en redes no es trivial. En primer lugar, porque el número de posibles particiones es muy elevado para redes no triviales, y en segundo lugar, porque el concepto de comunidad puede ser distinto según el dominio concreto de aplicación (Danon et al., 2005).

El algoritmo de Girvan-Newman (2002), empleado en nuestra investigación, permite separar los distintos componentes de una comunidad. Para ello, se calcula la betweenness de todos los enlaces de la red, y se elimina aquel que tenga mayor betweenness, repitiendo el proceso hasta que no quede ningún enlace. El resultado de este algoritmo es un dendograma donde las diferentes secciones horizontales representan distintas divisiones en comunidades, dependiendo del número deseado de comunidades. Sin embargo, este algoritmo no proporciona el número apropiado de comunidades para dividir la red. Por esta razón, Girvan y Newman proponen un método para evaluar las posibles particiones utilizando para ello el concepto de modularidad (Newman \& Girvan, 2004). La modularidad ${ }^{20}$ de una partición es un índice que cuantifica lo buena que es la partición: las particiones con altos valores de modularidad son aquellas en las que existen conexiones densas entre los nodos que forman parte del clúster pero con conexiones escasas entre clústeres.

La filosofía del algoritmo es el hecho de que los enlaces que conectan comunidades tienen una alta betweenness en comparación con el resto de los enlaces de la red, por lo que, al ir eliminando enlaces iterativamente, el último enlace en ser eliminado será el que separa los distintos componentes de la red.

${ }^{20}$ La modularidad compara el número de enlaces que existen dentro de una comunidad con el número de enlaces que habría si los enlaces entre nodos fueran generados aleatoriamente manteniendo el grado de cada nodo. 


\subsubsection{MODELOS TEÓRICOS DE REDES}

A continuación presentamos algunos modelos teóricos de redes y justificamos el tipo de red que hemos escogido en nuestra investigación.

\subsubsection{MODELO DE ERDOS-RENYI (Red Aleatoria)}

Este modelo fue propuesto por los matemáticos Paul Erdős y Alfréd Rényi (1959). Las redes construidas de esta manera también reciben el nombre de redes aleatorias o grafos aleatorios.

Para construir este tipo de red, se parte de un número fijo de nodos, N. A continuación, se van eligiendo pares de nodos al azar y se tiende un enlace entre ellos. Este proceso se repite $M$ veces, escogiendo aleatoriamente una pareja de nodos cada vez. Durante el proceso, se pueden escoger nodos que ya estén enlazados con otros nodos, pero no se pueden seleccionar parejas de nodos que ya estén enlazados. Al finalizar el proceso, si el número de enlaces $\mathrm{M}$ es considerablemente superior al número de nodos $\mathrm{N}$, se obtiene una red en la que prácticamente la totalidad de los nodos están conectados, formando un componente gigante (Solé, 2009). La Figura 13 muestra una red de este tipo.

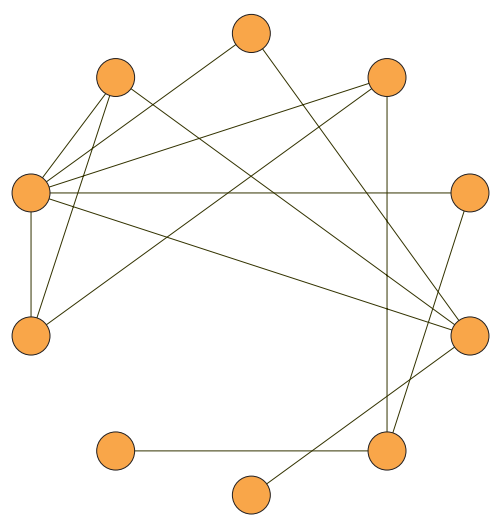

Figura 13. Red de Erdős y Rényi con $\mathrm{N}=10$ nodos y $\mathrm{M}=15$ enlaces. 
Las redes de Erdős y Rényi tienen una serie de propiedades:

- Erdős y Rényi demostraron que 1 era el número medio de enlaces por nodo necesario para que emergiera el gran clúster en el que la mayoría de los nodos de la red están interconectados.

- Si se aumenta el número medio de enlaces por nodo, el número de nodos aislados decrece exponencialmente.

- Las redes de Erdős y Rényi poseen la propiedad de pequeño-mundo, es decir, la distancia geodésica entre dos nodos es pequeña.

- La distribución de grado de una red de Erdős y Rényi presenta un pico predominante, lo cual indica que la mayoría de los nodos tienen el mismo número de enlaces, mientras que a ambos lados de este pico, el número de enlaces decrece rápidamente. Es decir, su distribución de grado es binomial, o de Poisson en el límite de una red suficientemente grande. La Figura 14 muestra la distribución de grado de la red aleatoria de la Figura 13.

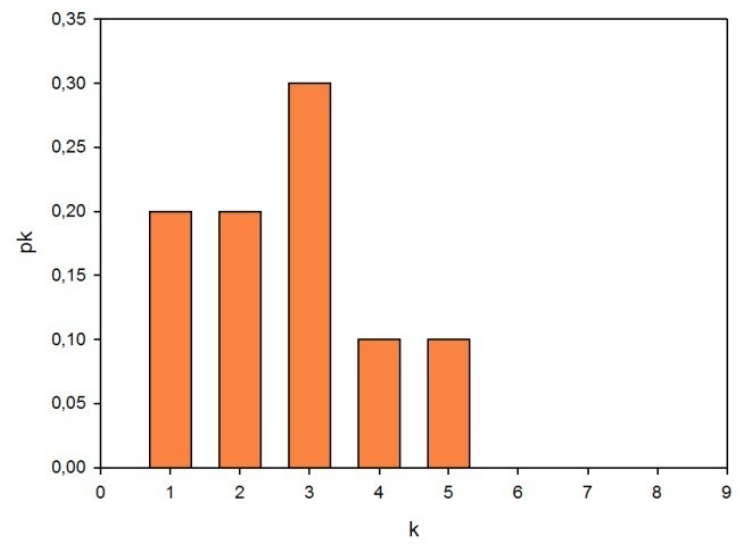

Figura 14. Distribución de grado de la red aleatoria de la Figura 13.

A pesar de que los trabajos de Erdős y Rényi supusieron una contribución importante para modelar las redes sociales, lo cierto es que redes aleatorias están lejos de ser una representación realista de las redes que se observan en la naturaleza (Barabási, 2002). La razón está en que, como ya hemos visto, las redes sociales presentan, además de la 
propiedad de pequeño-mundo, otra característica fundamental, conocida como alto clustering, y las redes aleatorias carecen de esta propiedad. Según el modelo de Erdős y Rényi, la probabilidad de que dos personas tengan un amigo en común es remota.

\subsubsection{RED REGULAR}

Se trata de una red en la que el número de enlaces por nodo es igual para todos los nodos de la red. Para construirla, simplemente se crean N nodos, cada uno de ellos con k enlaces, que les unirán a sus k vecinos más cercanos (ver Figura 15). Las redes regulares son muy frecuentes en la organización cristalina de los sistemas inertes y también en sistemas diseñados artificialmente (Solé, 2009).

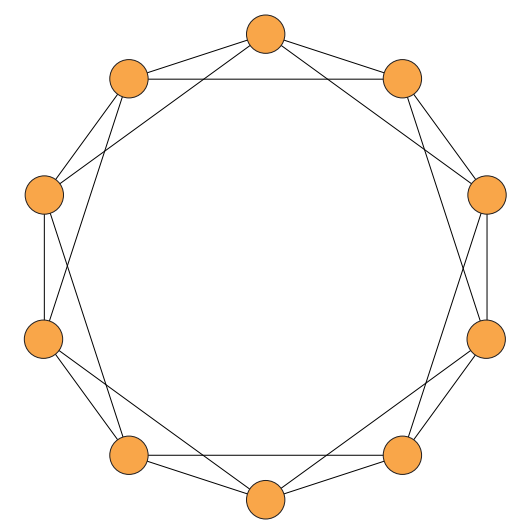

Figura 15. Red Regular con 10 nodos y grado medio $=4$.

Al contrario de lo que ocurre con los grafos aleatorios, las redes regulares se caracterizan por una distancia geodésica elevada, por lo que carecen de la propiedad de pequeñomundo. No obstante, el clustering que presenta este tipo de redes es elevado.

La distribución de grado de la red regular de la Figura 15 se muestra en la Figura 16. Dado que en una red regular todos los nodos tienen el mismo número de enlaces, la distribución de grado toma un único valor $\left(p_{k}=1\right)$ cuando $k$ es igual al número medio de enlaces por nodo (4 en el caso de la red de la Figura 15) y cero para el resto de valores de $k$. 


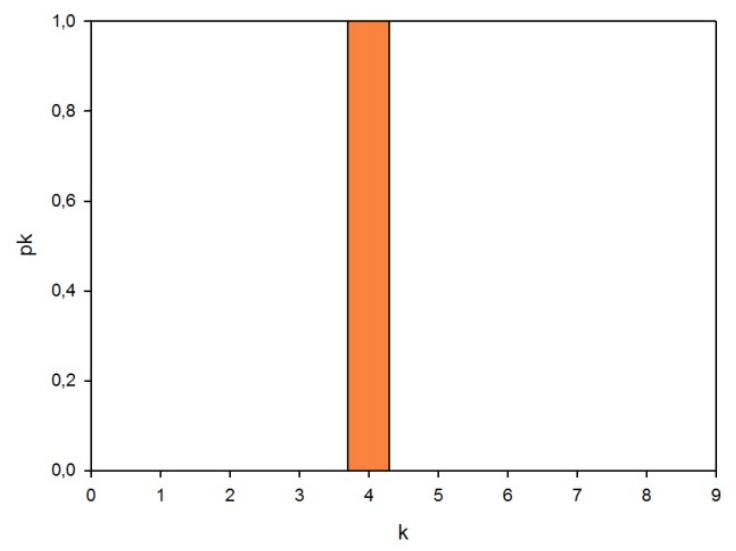

Figura 16. Distribución de grado de la red regular mostrada en la Figura 15.

Lo deseable a la hora de modelar una red social sería encontrar un modelo de red que combinara, entre otras, las propiedades de pequeño-mundo (como la red aleatoria) y la propiedad de alto clustering (como la red regular). Un modelo de red que cumple ambas propiedades a la vez es el modelo de Watts-Strogatz.

\subsubsection{RED DE WATTS-STROGATZ}

Una red de Watts-Strogatz (1998) está caracterizada por tres parámetros: N (número de nodos), $\mathrm{k}$ (grado o número de enlaces por nodo) y $\beta$ (probabilidad de rewiring).

Para construir una red de Watts-Strogatz, se parte de una red regular, con N nodos y grado $k$. A continuación recorremos uno a uno los $\mathrm{N}$ nodos de la red. En cada uno de los nodos, vamos tomando cada uno de sus enlaces, y con cierta probabilidad (que vendrá dada por el parámetro $\beta$, llamado probabilidad de rewiring) sustituimos la conexión actual del enlace por la conexión con otro nodo de la red tomado al azar (no se permite que el nuevo enlace del nodo se haga consigo mismo). El proceso se repite con el resto de los nodos de la red. 
En la Figura 17 se muestra una red de Watts-Strogatz con 10 nodos, grado medio 4 y probabilidad de rewiring $\beta=0,1$. Para su construcción, se ha partido de la red regular de la Figura 15.

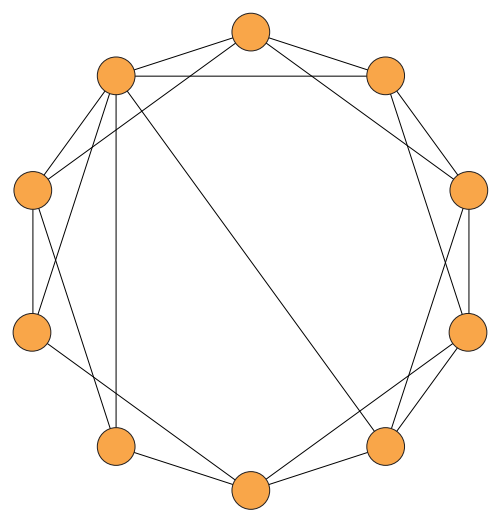

Figura 17. Red de Watts-Strogatz con 10 nodos, grado medio $=4$ y probabilidad de rewiring $\beta$ $=0,1$.

Las propiedades de las redes generadas mediante este algoritmo dependen de la probabilidad de rewiring. Para el caso concreto de probabilidad de rewiring $\beta=0$, se obtiene una red regular (con alto clustering pero sin la propiedad de pequeño-mundo). Para el caso de probabilidad de rewiring $\beta=1$, lo que resulta es una red aleatoria (con bajo clustering aunque con la propiedad de pequeño-mundo).

Conforme $\beta$ aumenta se incrementa la probabilidad de que aparezcan enlaces que comunican lugares distantes dentro de la red, haciendo que la distancia geodésica disminuya y, consecuentemente, propiciando la aparición del efecto de pequeño-mundo. Del mismo modo, al aumentar $\beta$, debido a la aparición de enlaces de larga distancia, también va disminuyendo el coeficiente de clustering de la red. Sin embargo, para valores intermedios de $\beta$, pueden obtenerse redes que presentan simultáneamente las propiedades de pequeño-mundo y de alto clustering.

La Figura 18 muestra el aspecto que ofrece una red de Watts-Strogatz con 10 nodos y grado medio 4 para diversos valores de $\beta$. 


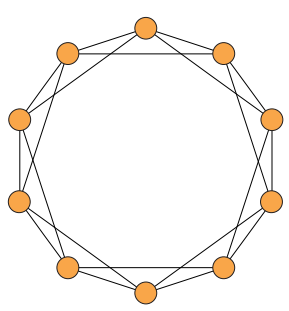

(a)

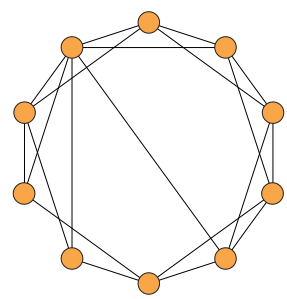

(b)

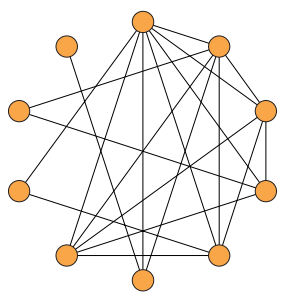

(c)

\section{Clustering}

Pequeño-mundo $\beta=0$

Alto

No $\beta=0.1$

Alto

Sí $\beta=1$

Bajo

Sí

Figura 18. Red de Watts-Strogatz con 10 nodos y grado medio 4, varios $\beta$.

Como puede observarse, en los casos extremos $\beta=0$ (Figura 18a) y $\beta=1$ (Figura 18c), se obtienen respectivamente una red regular y una red aleatoria. Sin embargo, para ciertos valores de $\beta$ en el rango $0<\beta<1$, se obtiene una red que presenta simultáneamente alto clustering y el efecto de pequeño mundo ${ }^{21}$. La red que se muestra en Figura $18 \mathrm{~b}$ ha sido generada con valor de $\beta=0,1$.

En la Figura 19 se representa el coeficiente de clustering normalizado ${ }^{22}$ y la distancia geodésica normalizada ${ }^{23}$ para una red de 1.000 nodos y grado medio 10 , en función de $\beta$.

${ }^{21}$ Esto es cierto para valores de $\mathrm{N}$ grandes. En la Figura 18 se ha empleado un número de nodos pequeño $(\mathrm{N}=10)$ por motivos de claridad.

${ }^{22}$ Para normalizar el coeficiente de clustering, lo dividimos entre su valor máximo, que ocurre para $\beta=0$.

${ }^{23}$ Para normalizar la distancia geodésica, la dividimos entre su valor máximo, que ocurre para $\beta=0$. 


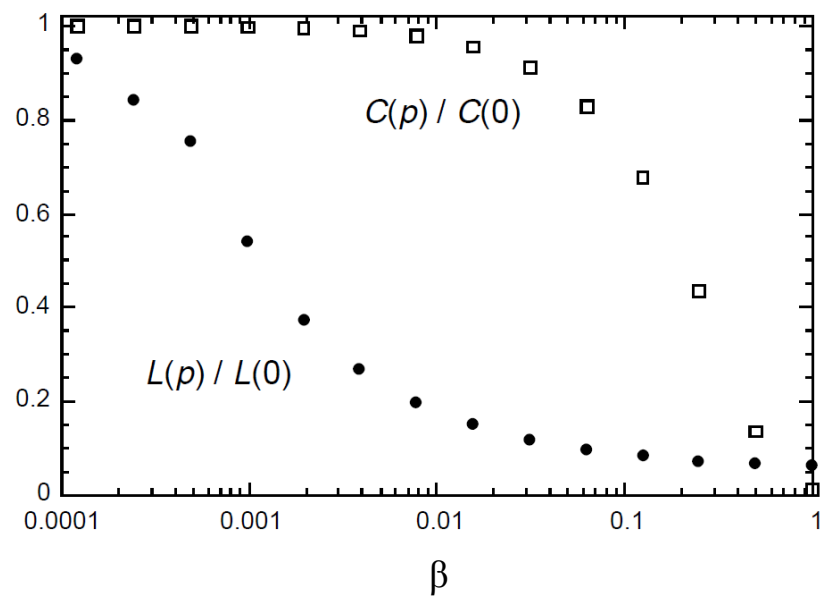

Figura 19. Coeficiente de clustering normalizado y distancia geodésica normalizada en función $\beta$ en una red de Watts-Strogatz con N=1.000 nodos y grado medio k=10 (Watts \& Strogatz, 1998).

La Figura 19 nos permite comprobar que existe un rango de valores de $\beta$ para los cuales la red presenta simultáneamente alto clustering y baja distancia geodésica, situación que se da en una amplia variedad de redes reales (Watts, 1999).

\subsubsection{MODELO DE BARABÁSI-ALBERT (o de conexión preferencial)}

El Modelo de Barabási-Albert (1999) parte de la observación de que algunas redes presentan nodos con un número de enlaces excepcionalmente alto en comparación con el resto de nodos de la red. Este tipo de nodos con elevado número de conexiones recibió el nombre de $h u b s^{24}$. Existen muchas redes en las que los hubs están presentes, como por ejemplo redes de aeropuertos, de actores, internet, etc (Newman, 2003).

La Figura 20 muestra una red de Barabási-Albert, en la que puede observarse la presencia de un nodo - denominado $h u b$ - en el que el número de conexiones es considerablemente superior a la del resto de los nodos de la red.

${ }^{24}$ En castellano, conector. 


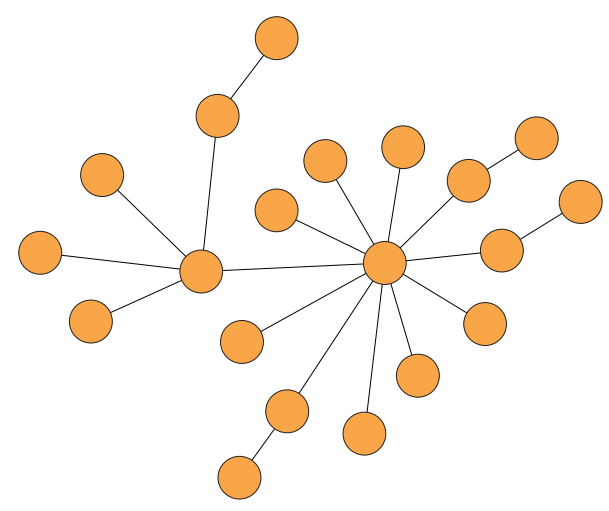

Figura 20. Red de Barabási-Albert con $\mathrm{N}=20$ nodos.

Las redes de Barabási-Albert tienen la propiedad de ser redes libres de escala (scale-free networks). Esto implica que su distribución de grado (es decir, histograma en el que se representa el número de nodos que tienen un determinado número de vecinos) sigue una ley de potencias (power law), fenómeno que no ocurría ni en el modelo de Erdős y Rényi ni en el de Watts-Strogatz. Informalmente, esto equivale a decir que, en este tipo de redes, existe una gran mayoría de nodos que tienen un número reducido de enlaces que convive con un número reducido de nodos que tienen un número de enlaces excepcionalmente elevado. Esto puede comprobarse en la Figura 21, que muestra la distribución de grado de la red de la Figura 20.

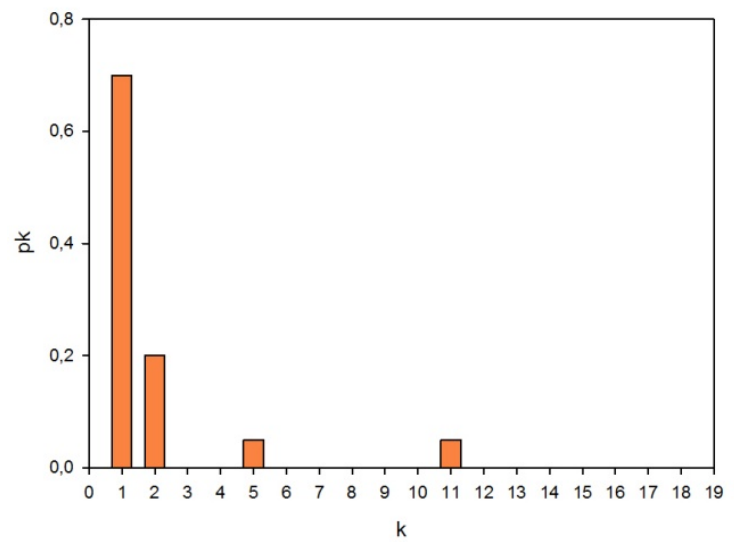

Figura 21. Distribución de grado de la red de Barabási-Albert de la Figura 20. 
Tanto en las redes aleatorias de Erdős y Rényi como en las redes de Watts-Strogatz todos los nodos son iguales en cuanto a la probabilidad de recibir nuevos enlaces durante la construcción de la red. Este hecho imposibilitaba la aparición de hubs en estos modelos.

El modelo de Barabási-Albert, sin embargo, permite construir redes libres de escala utilizando para ello la llamada conexión preferencial (preferential attachment). El mecanismo que se sigue es el siguiente: a la hora de construir la red, en lugar de colocar todos los nodos en una sola vez y después comenzar a tender enlaces (como se hace en los modelos de Erdős y Rényi y de Watts-Strogatz), tanto los nodos como los enlaces se van añadiendo paso a paso durante la construcción de la red. En cada paso, se añade un nuevo nodo al sistema, y se tiende un enlace a otro nodo de la red. De esta manera se consigue que los nodos que se añadieron en primer lugar reciban más enlaces que los que van llegando en último lugar, simplemente porque llevan más tiempo en el sistema. Por otro lado, cada vez que se introducen un nuevo nodo y enlace en el sistema, el nuevo enlace no tiene la misma probabilidad de conectar con cualquier nodo del sistema, sino que tendrá mayor preferencia por algunos nodos que por otros: la probabilidad de que el nuevo nodo escoja a un nodo dado es proporcional al número de enlaces del nodo objetivo. Esto es lo que Barabási y Albert denominaron conexión preferencial (preferential attachment).

El modelo original Barabási-Albert que acabamos de describir explica la aparición de hubs. Sin embargo, presenta el inconveniente de que el nodo más antiguo tiene más posibilidades de recibir nuevos enlaces simplemente por el hecho de llevar más tiempo en el sistema. Esta peculiaridad impide que los nodos que han sido introducidos recientemente en el sistema puedan convertirse en hubs. Por esta razón, el modelo fue refinado posteriormente por Bianconi y Barabási (2001)25, asociando a los nodos una propiedad adicional (fitness). Cada nodo tiene una fitness determinada, de tal modo que, en esta nueva versión del modelo, la probabilidad de que un nodo reciba un nuevo enlace es proporcional al producto de su fitness por el número de enlaces del nodo.

${ }^{25}$ El modelo de fitness también recibe el nombre de modelo de Bianconi-Barabási. 


\section{EL JUEGO DE LA DEMANDA DE $\mathrm{NASH}$}

\subsection{INTRODUCCIÓN}

\subsubsection{CONCEPTO DE EXCEDENTE}

El Juego de la demanda de Nash es una abstracción de una situación de negociación en la que existe cierto excedente a repartir entre dos individuos. Un ejemplo de este tipo de situaciones podemos encontrarlo en un entorno de compra-venta en el que el comprador no sabe el precio mínimo al que está dispuesto a vender el vendedor, y el vendedor desconoce el precio máximo que está dispuesto a pagar el comprador. Supongamos que el valor que tiene el bien para el vendedor es de $10 €$, y para el comprador de $15 €$. El comprador no está dispuesto a vender el bien por menos de $10 €$, pero el comprador no está dispuesto a pagar más de $15 €$. Es decir, para que la transacción se lleve a cabo, el precio de compra-venta deberá estar comprendido entre $10 €$ y $15 €$. La diferencia entre estos valores $(5 €)$ será el excedente que comprador y vendedor deberán negociar (Figura $22)$.

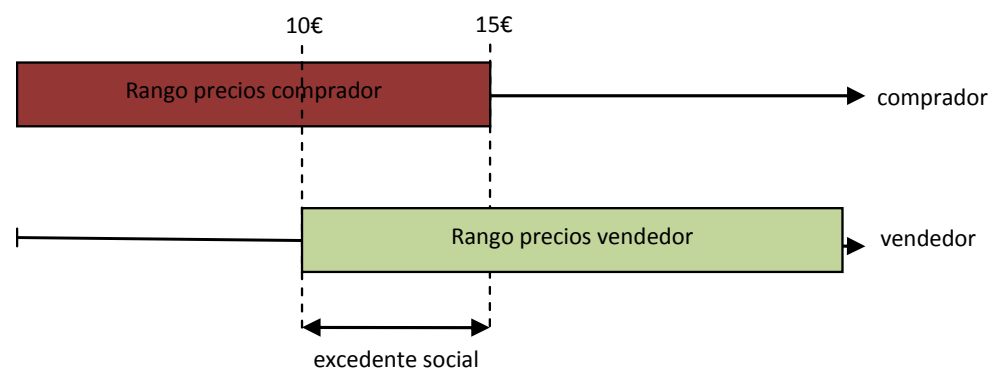

Figura 22. Rangos de precios a los que puede efectuarse la transacción y excedente a repartir. 
Como resultado de la negociación, pueden darse tres situaciones:

- Que el vendedor proponga un precio superior a $15 €$ o bien que el comprador ofrezca un precio inferior a $10 €$. Ante esta situación, no hay intercambio.

- Que tanto el comprador como el vendedor el vendedor propongan un precio comprendido entre $10 €$ y $15 €$. En este, caso el intercambio tendría lugar, y habría un excedente social de $5 €$ a repartir entre el comprador y el vendedor. Dentro de este caso, distinguimos dos situaciones:

- Reparto paritario. Se produce cuando el precio de venta es $12,5 €$. El excedente se reparte a la mitad y, como consecuencia, el excedente del consumidor y el excedente del vendedor coinciden (ver Figura 23).

- Reparto discriminatorio. Se produce para precios de venta distintos a $12,5 €$ dentro del rango 10-15€. En este caso, uno de los dos individuos obtiene una mayor cantidad del excedente que el otro. El excedente del vendedor y del consumidor no coinciden (ver Figura 24).

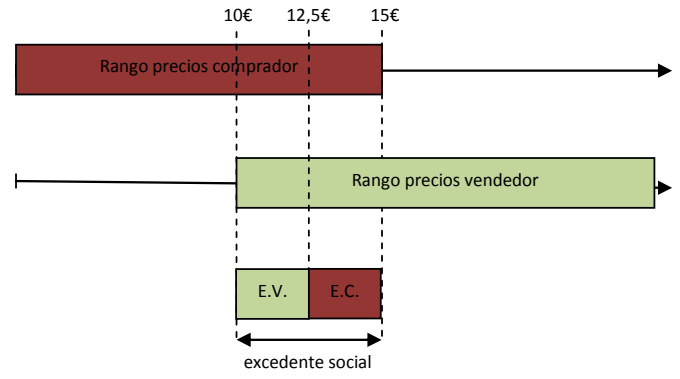

Figura 23. Reparto paritario del excedente.

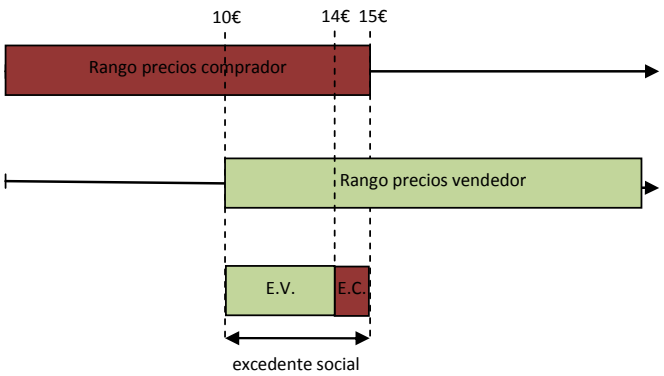

Figura 24. Reparto discriminatorio del excedente.

Esta situación de reparto de excedente puede modelarse mediante un juego en el que dos agentes negocian cierta cantidad de una propiedad (en nuestro ejemplo, el excedente social) y cada uno de los agentes demanda cierta cantidad de la misma. En la negociación puede ocurrir que la suma de las dos demandas sea superior a la cantidad del bien que se está repartiendo. Por ejemplo, si el comprador desea un excedente de $4 €$ (precio $11 €$ ) y el vendedor desea un excedente de $2 €$ (precio de $12 €$ ), la suma de ambas demandas $(6 €)$ es superior al excedente $(5 €)$ y el intercambio no se produce. Por el contrario, también puede ocurrir que la suma de las dos demandas sea inferior al 
excedente que se está repartiendo. En este caso el intercambio sí se produce y, además, cada individuo recibe la parte de excedente que ha demandado ${ }^{26}$.

Este juego de negociación, en el que dos agentes demandan cierta cantidad de un recurso a repartir y reciben lo que han pedido siempre que la suma de ambas demandas sea inferior a la cantidad total del recurso a repartir, y nada en el caso contrario, fue propuesto por Nash en su artículo Two-person Cooperative Games (1953). Posteriormente, este juego pasaría a la historia como juego de la demanda de Nash.

\subsubsection{DESCRIPCIÓN}

Desde una perspectiva ligeramente más formal, el juego de la demanda de Nash consiste en lo siguiente: sean dos agentes que demandan cierta cantidad de una propiedad. Supongamos, por simplicidad, que la cantidad de la propiedad a repartir es unitaria (1). Sean $i$ y $j$, respectivamente, las cantidades de la propiedad que demandan el primer y el segundo jugador. La cantidad de la propiedad que recibe cada uno de los agentes es la siguiente:

Jugador 1 recibe $i$ y Jugador 2 recibe $j$ siempre que $i+j \leq 1$

Jugador 1 recibe 0 y Jugador 2 recibe 0 si $i+j>1$

\subsubsection{ANTECEDENTES}

Hasta la década de 1950 se consideraba que las negociaciones bilaterales como la descrita en la sección 3.1.1 no tenían un resultado claramente predecible, y se pensaba que el

${ }^{26}$ Estamos suponiendo que tanto el comprador como el vendedor dan a conocer su propuesta de precio simultáneamente, por lo que en caso de que los precios sean compatibles (es decir, que la suma de las dos demandas sea inferior al excedente a repartir), tanto el comprador como el vendedor deberán efectuar la transacción al precio que cada uno de ellos ha propuesto. 
reparto del excedente dependía solamente de la capacidad de negociación de ambas partes (Anbarci \& Feltovich, 2010).

Previamente a los aportes de John F. Nash, en los primeros años de la década de 1950, la Teoría de Juegos se basaba fundamentalmente en los estudios de Neumann y Morgenstern (1944), quienes proporcionaron una solución para este tipo de negociación. Sin embargo, esta solución se limitaba a juegos de dos jugadores y suma-cero, mientras que su teoría estaba prácticamente vacía para juegos de suma no-cero y para los juegos de n-jugadores (Watson, 2009). Por un lado, para tratar juegos de más de dos jugadores, recurrían a coaliciones de jugadores, de tal forma que la situación pudiera tratarse como un juego de dos jugadores. Por otro lado, para tratar los juegos de suma no-cero debía recurrirse a un tercer jugador ficticio cuyo pago era la suma de los pagos de los dos jugadores reales cambiada de signo, obteniendo así un juego de suma-cero.

John F. Nash (1950) superó estas limitaciones y propuso un concepto de equilibrio (que posteriormente pasaría a la Historia como equilibrio de Nash) que permitía predecir el resultado del juego sin necesidad de recurrir a hipótesis de suma-cero ni de coalición de agentes. Para ello, Nash idealizó el problema de negociación asumiendo que los dos individuos son altamente racionales, que cada uno puede comparar de forma precisa sus deseos por diversos bienes, que son iguales en técnicas de negociación y que cada uno tiene completo conocimiento sobre los gustos y preferencias del otro.

Posteriormente, en su artículo Two-person Cooperative Games (1953), propuso su juego de la demanda de Nash, en el que dos jugadores reciben la porción que solicitan de un bien siempre que la suma de ambas demandas sea igual o inferior a la cantidad de bien a repartir.

El dilema que se plantea es el siguiente: las demandas grandes producen recompensas grandes pero la probabilidad de obtenerlas es baja, ya que corremos el riesgo de no recibir nada si el otro individuo también demanda una cantidad grande del recurso; por otro lado, las demandas bajas producen recompensas bajas, pero la probabilidad de obtener la cantidad demandada es mayor, ya que contribuimos, por nuestra parte, a que la suma de ambas demandas sea inferior a la cantidad de recurso a repartir. 


\subsubsection{MODIFICACIONES EN EL JUEGO DE LA DEMANDA DE NASH}

El juego de la demanda de Nash ha sido sometido a numerosos refinamientos, modificaciones y extensiones en las últimas décadas.

Rubinstein (1982) introdujo un modelo en el que dos agentes debían llegar a un acuerdo para repartir una "tarta" de tamaño 1. Mientras que en el modelo original de Nash (1953) ambos agentes daban a conocer sus demandas simultáneamente, en el modelo de Rubinstein los agentes iban formulando sus demandas alternativamente. Después de que un jugador hubiera hecho una oferta, el otro jugador debía decidir si aceptaba el reparto propuesto por el otro jugador o bien si lo rechazaba y proponía otra división. Tanto el modelo de Rubinstein como el de Nash son juegos de dos jugadores y una única interacción entre ellos (one-shot games) en el que se asume que los jugadores disponen de racionalidad ilimitada.

Posteriormente, Young (1993) propone una versión evolutiva del juego de la demanda de Nash en la que una población finita de agentes es emparejada al azar para jugar sucesivamente al juego de la demanda de Nash. En contraste con los modelos de Nash y de Rubinstein, que eran juegos de una única interacción entre agentes (one-shot games), el modelo de Young es un juego repetitivo, en el que los agentes se van emparejando aleatoriamente y de forma sucesiva a lo largo de varias rondas. Esto permite a los agentes ir aprendiendo a lo largo del juego cuál es la estrategia que les permite obtener mayor beneficio. Para ello, los agentes van almacenando en su memoria las decisiones tomadas por sus oponentes en encuentros anteriores. En este modelo, los agentes pueden escoger entre una serie de demandas comprendidas entre 0 y 1 que están separadas entre sí cierta cantidad regulada por un parámetro. El hecho de convertir el juego de la demanda de Nash en un juego repetitivo permitió a Young observar la dinámica de la población y estudiar los puntos de atracción hacia los que evoluciona el sistema.

Posteriormente, Axtell, Epstein y Young (2000) llevaron a cabo una extensión del modelo de Young (1993) al que llamaron Modelo de Clases. Simplificaron el análisis considerando que las estrategias de los agentes (es decir, las posibles demandas del recurso a repartir) se limitaban a tres: una porción pequeña (30\%), mediana (50\%) o grande (70\%). Además, introdujeron dos clases de agentes en el sistema y analizaron las 
normas que pueden emerger a partir de la interacción entre las distintas clases de agentes. El Modelo de Clases, que es precisamente el punto de partida de este trabajo de investigación, se encuentra descrito en la sección 3.2.

En (Poza et al., 2011) se lleva a cabo una réplica de este Modelo de Clases, obteniendo resultados en consonancia con los que se obtenían en aquel modelo, tal como Dessalles (2007) había confirmado en una replicación previa de este mismo modelo. En este trabajo también se han llevado a cabo algunas extensiones al modelo original, como cambios en la matriz de pagos del juego (ver sección 3.3.1), cambios en las condiciones iniciales del sistema (ver sección 3.3.2) y cambios en la regla de decisión de los agentes (tal como se sugería en López-Paredes et al. 2004), ver sección 3.3.3. El artículo (Poza et al., 2011), publicado en la revista Discrete Dynamics in Nature and Society se ha incluido en el bloque 2 de esta tesis doctoral.

En un reciente trabajo, (Ohtsuki, 2011), el juego de la demanda de Nash es implementado desde una aproximación evolutiva. En este modelo, existen dos poblaciones, cada una de las cuales sigue una estrategia. Los agentes de una especie son emparejados al azar para interactuar con los agentes de la otra especie y llevar a cabo el juego de la demanda de Nash. El resultado del juego afecta a la tasa de reproducción de los individuos, de manera que los jugadores que obtienen un pago mayor obtienen una descendencia mayor, lo cual facilita que, en futuras generaciones, la especie que obtiene mejores pagos en el juego tenga un número de individuos mayor que la especie que tiene una estrategia menos exitosa. Por otro lado, en este modelo, el campo de posibles estrategias para los agentes es un continuo entre 0 y 1 , lo cual se traduce en un número infinito de posibles equilibrios de Nash.

A pesar de que en el Modelo de Clases (Axtell, Epstein \& Young, 2000), el emparejamiento entre agentes era aleatorio, una de las extensiones que llevamos a cabo consistió en situar a los agentes sobre un grid regular, de tal forma que la interacción entre agentes (y, por tanto, el aprendizaje) se diera solamente entre agentes vecinos (ver sección 3.4). Dicha extensión, (Poza et al., 2011) fue publicada en la revista PLoS ONE, y se ha incluido en el bloque 2 de esta tesis doctoral. La razón de introducir esta modificación fue que algunas investigaciones sugieren que el hecho de que las interacciones tengan lugar entre vecinos puede provocar un comportamiento dinámico muy diferente al que se observa cuando los emparejamientos son aleatorios (Alexander \& Skyrms, 1999; Pollock, 1989). Una de las primeras investigaciones que tuvieron en 
cuenta estas limitaciones topológicas fue el trabajo de Nowak y May (1992), quienes estudiaron el dilema del prisionero cuando los agentes están situados sobre un grid.

En (Alexander \& Skyrms, 1999) también se estudia el juego de la demanda de Nash en un grid. Sin embargo, existen ciertas diferencias en cuanto a la toma de decisiones de los agentes. En el trabajo de Alexander y Skyrms, los agentes no tienen memoria, por lo que no basan sus decisiones en función de las decisiones tomadas por sus oponentes en el pasado, sino que simplemente imitan la estrategia del oponente que ha obtenido mejor resultados que ellos en el juego (si es que lo hay). También existen diferencias en cuanto a la matriz de pagos. Mientras que en nuestro modelo existen tres posibles demandas (low, medium y high), lo cual da lugar a tres equilibrios de Nash, en el trabajo de Alexander y Skyrms se tienen nueve posibles demandas (10, 20, ..., 90), dando lugar a nueve equilibrios de Nash distintos. Con este planteamiento, los autores concluyen que el hecho de situar a los agentes en un grid favorece la emergencia de la norma paritaria.

En una tercera extensión, (Santos et al., 2012), se dio un paso más allá hacia un modelo más realista en cuanto a la interacción entre agentes, integrándolos en una red social y haciendo que éstos interactúen solamente con sus vecinos dentro la red (ver sección 3.5). Dicho trabajo, publicado en la revista Discrete Dynamics in Nature and Society, se ha incluido en el bloque 2 de esta tesis doctoral.

En (Gallo, 2011), también se utilizan conjuntamente el juego de la demanda de Nash y la Teoría sobre Redes Sociales. Sin embargo, existen destacables diferencias entre este trabajo y nuestra investigación. Por un lado, en el modelo de Gallo, el número de posibles pagos que reciben los agentes es variable (en función de un parámetro $\delta$, al igual que en el modelo de Young (1993), mientras que en nuestro modelo sólo existen tres posibles pagos (low, medium y high), siguiendo el Modelo de Clases de Axtell, Epstein y Young (2000). En lo que se refiere a redes sociales, en nuestro trabajo, la red limita los vecinos con los que cada agente puede interactuar (es decir, con los que pueden efectuar el juego de la demanda de Nash y de los cuales pueden aprender). Sin embargo, en el modelo de Gallo, la integración de los agentes en una red social solamente afecta a la manera en la que fluye la información entre los agentes, pero no a las negociaciones (para esto último, los agentes siguen siendo emparejados aleatoriamente).

En la Tabla 10 se muestran algunas de las principales extensiones del juego de la demanda de Nash que se han llevado a cabo a lo largo del tiempo. 


\begin{tabular}{|c|c|c|c|c|c|}
\hline Trabajo & $\begin{array}{c}\text { Variante } \\
\text { T.J. }\end{array}$ & Decisión & $\begin{array}{c}\text { Regla } \\
\text { decisión }\end{array}$ & Topología & Demandas \\
\hline Nash, 1953 & $\begin{array}{l}\text { Clásica } \\
\text { (one shot) }\end{array}$ & Simultánea & $\begin{array}{l}\text { Racionalidad } \\
\text { perfecta }\end{array}$ & $\begin{array}{l}\text { No } \\
\text { (emparejamiento } \\
\text { aleatorio) }\end{array}$ & $\begin{array}{l}\text { Continuas } \\
\text { entre } 0 \text { y } 1\end{array}$ \\
\hline $\begin{array}{l}\text { Rubinstein, } \\
1982\end{array}$ & $\begin{array}{l}\text { Clásica } \\
\text { (one shot) }\end{array}$ & Secuencial & $\begin{array}{l}\text { Racionalidad } \\
\text { perfecta }\end{array}$ & $\begin{array}{l}\text { No } \\
\text { (emparejamiento } \\
\text { aleatorio) }\end{array}$ & $\begin{array}{l}\text { Continuas } \\
\text { entre } 0 \text { y } 1\end{array}$ \\
\hline $\begin{array}{l}\text { Young, } \\
1993\end{array}$ & $\begin{array}{l}\text { Evolutiva / } \\
\text { Aprendizaje }\end{array}$ & Simultánea & $\begin{array}{l}\text { Racionalidad } \\
\text { (casi) } \\
\text { perfecta }\end{array}$ & $\begin{array}{l}\text { No } \\
\text { (emparejamiento } \\
\text { aleatorio) }\end{array}$ & $\begin{array}{l}\text { Discretas } \\
\text { entre } 0 \text { y } 1 \\
\text { (separación } \\
\delta \text { entre } \\
\text { demandas) }\end{array}$ \\
\hline $\begin{array}{l}\text { Alexander } \\
\text { \& Skyrms, } \\
1999\end{array}$ & $\begin{array}{l}\text { Evolutiva / } \\
\text { aprendizaje }\end{array}$ & Simultánea & $\begin{array}{l}\text { Aprendizaje } \\
\text { por } \\
\text { imitación }\end{array}$ & Grid regular & $\begin{array}{l}\text { Continuas } \\
\text { entre } 0 \text { y } 1\end{array}$ \\
\hline $\begin{array}{l}\text { Axtell et } \\
\text { al., } 2000\end{array}$ & $\begin{array}{l}\text { Evolutiva / } \\
\text { Aprendizaje }\end{array}$ & Simultánea & $\begin{array}{l}\text { Racionalidad } \\
\text { (casi) } \\
\text { perfecta }\end{array}$ & $\begin{array}{l}\text { No } \\
\text { (emparejamiento } \\
\text { aleatorio) }\end{array}$ & $\begin{array}{l}\operatorname{low}(0.3) \\
\text { medium }(0.5) \\
\operatorname{high}(0.7) \\
\end{array}$ \\
\hline $\begin{array}{l}\text { López- } \\
\text { Paredes et } \\
\text { al., } 2004 \\
\end{array}$ & $\begin{array}{l}\text { Evolutiva / } \\
\text { Aprendizaje }\end{array}$ & Simultánea & $\begin{array}{l}\text { Racional / } \\
\text { Fast and } \\
\text { Frugal } \\
\end{array}$ & $\begin{array}{l}\text { No } \\
\text { (emparejamiento } \\
\text { aleatorio) } \\
\end{array}$ & $\begin{array}{l}\operatorname{low}(0.3) \\
\text { medium }(0.5) \\
\operatorname{high}(0.7) \\
\end{array}$ \\
\hline $\begin{array}{l}\text { Dessalles, } \\
2007\end{array}$ & $\begin{array}{l}\text { Evolutiva / } \\
\text { Aprendizaje }\end{array}$ & Simultánea & $\begin{array}{l}\text { Racionalidad } \\
\text { (casi) } \\
\text { perfecta }\end{array}$ & $\begin{array}{l}\text { No } \\
\text { (emparejamiento } \\
\text { aleatorio) }\end{array}$ & $\begin{array}{l}\operatorname{low}(0.3) \\
\text { medium }(0.5) \\
\operatorname{high}(0.7) \\
\end{array}$ \\
\hline $\begin{array}{l}\text { Ohtsuki, } \\
2011\end{array}$ & Evolutiva & Simultánea & $\begin{array}{l}\text { Dependiente } \\
\text { de la especie }\end{array}$ & $\begin{array}{l}\text { No } \\
\text { (emparejamiento } \\
\text { aleatorio) }\end{array}$ & $\begin{array}{l}\text { Continuas } \\
\text { entre } 0 \text { y } 1\end{array}$ \\
\hline Gallo, 2011 & $\begin{array}{l}\text { Evolutiva / } \\
\text { Aprendizaje }\end{array}$ & Simultánea & $\begin{array}{l}\text { Racionalidad } \\
\text { (casi) } \\
\text { perfecta }\end{array}$ & $\begin{array}{l}\text { Red social (para } \\
\text { flujo de } \\
\text { información) / } \\
\text { aleatorio (para } \\
\text { interacciones) }\end{array}$ & $\begin{array}{l}\text { Discretas } \\
\text { entre } 0 \text { y } 1 \\
\text { (separación } \\
\text { variable } \delta \\
\text { entre } \\
\text { demandas) } \\
\end{array}$ \\
\hline $\begin{array}{l}\text { Poza et al., } \\
2011\end{array}$ & $\begin{array}{l}\text { Evolutiva / } \\
\text { Aprendizaje }\end{array}$ & Simultánea & $\begin{array}{l}\text { Racional / } \\
\text { Fast and } \\
\text { Frugal } \\
\end{array}$ & $\begin{array}{l}\text { No } \\
\text { (emparejamiento } \\
\text { aleatorio) }\end{array}$ & $\begin{array}{l}\operatorname{low}(0.3) \\
\text { medium }(0.5) \\
\operatorname{high}(0.7) \\
\end{array}$ \\
\hline $\begin{array}{l}\text { Poza et al., } \\
2011\end{array}$ & $\begin{array}{l}\text { Evolutiva / } \\
\text { Aprendizaje }\end{array}$ & Simultánea & $\begin{array}{l}\text { Fast and } \\
\text { Frugal }\end{array}$ & Grid regular & $\begin{array}{l}\operatorname{low}(0.3) \\
\text { medium }(0.5) \\
\text { high }(0.7)\end{array}$ \\
\hline $\begin{array}{l}\text { Santos et } \\
\text { al., } 2012\end{array}$ & $\begin{array}{l}\text { Evolutiva / } \\
\text { Aprendizaje }\end{array}$ & Simultánea & $\begin{array}{l}\text { Fast and } \\
\text { Frugal }\end{array}$ & Red social & $\begin{array}{l}\operatorname{low}(0.3) \\
\text { medium }(0.5) \\
\operatorname{high}(0.7)\end{array}$ \\
\hline
\end{tabular}

Tabla 10. Diversas modificaciones del juego de la demanda de Nash y sus particularidades. 


\subsection{EL MODELO DE CLASES DE AXTELL, EPSTEIN Y YOUNG}

\subsubsection{DESCRIPCIÓN DEL MODELO}

Se parte de una población de $N$ agentes idénticos, que son emparejados aleatoriamente para llevar a cabo el juego de la demanda de Nash. En el Modelo de Clases de Axtell, Epstein y Young (2000), se utiliza una "tarta" como metáfora del recurso que se va a repartir entre los dos agentes. En cada interacción, cada jugador demandará cierta porción de tarta, según una regla de decisión que veremos posteriormente, a elegir entre tres porciones ${ }^{27}$ :

- $\quad \boldsymbol{L} \boldsymbol{o w}$ (el agente demanda una porción pequeña de la tarta).

- Medium (el agente demanda exactamente la mitad de la tarta).

- High (el agente demanda una porción grande de la tarta).

En lo sucesivo, nos referiremos a estas tres estrategias como L, M y H, respectivamente.

En el modelo de AEY, los valores asignados a L, M y H están particularizados para los valores 30\%, 50\% y 70\% respectivamente. Al igual que en el juego de la demanda de Nash original, cada jugador recibe la cantidad demandada siempre que la suma de las demandas de ambos jugadores no supere el cien por cien de la "tarta". En caso contrario, ninguno de los dos recibe nada. La matriz de pagos correspondiente se muestra en la Tabla 11.

\begin{tabular}{|c|l|c|c|c|}
\cline { 3 - 5 } \multicolumn{1}{c|}{} & \multicolumn{3}{c|}{ Jugador 2} \\
\cline { 2 - 5 } & $\mathrm{L}($ low) & $\mathrm{L}, \mathrm{L}$ & $\mathrm{L}, \mathrm{M}$ & $\mathrm{H}$ (high) \\
\cline { 2 - 5 } Jugador 1 H \\
\cline { 2 - 5 } & $\mathrm{M}$ (medium) & $\mathrm{M}, \mathrm{L}$ & $\mathbf{M}, \mathbf{M}$ & 0,0 \\
\cline { 2 - 5 } & $\mathrm{H}$ (high) & $\mathbf{H}, \mathbf{L}$ & 0,0 & 0,0 \\
\cline { 2 - 5 } & & &
\end{tabular}

Tabla 11. Matriz de pagos en el modelo de Axtell, Epstein y Young (2000).

${ }^{27}$ En el lenguaje de la Teoría de Juegos, cada agente tiene tres estrategias puras. 
Los tres resultados resaltados en la Tabla 11 se corresponden con los tres equilibrios de Nash en estrategias puras que posee el sistema (ver sección 2.2.3).

Cada jugador dispone de una memoria de tamaño $m$ en el que almacena las decisiones de cada uno de los $m$ últimos jugadores contra los que se ha enfrentado. A la hora de tomar una decisión, cada agente utiliza la información de su memoria para elegir la estrategia que maximiza su beneficio.

En concreto, lo que hacen los agentes es calcular el beneficio medio que obtendrían si eligieran L, M o H, para después escoger la estrategia que maximiza su beneficio esperado. Los beneficios esperados al escoger L, M y $\mathrm{H}$ según la matriz de pagos del juego (Tabla 11) son, respectivamente, los indicados en la Tabla 12.

$$
\begin{aligned}
& B_{L}=L \cdot p(L)+L \cdot p(M)+L \cdot p(H)=\mathrm{L} \\
& B_{M}=M \cdot p(L)+M \cdot p(M)+0 \cdot p(H)=\mathrm{M} \cdot(\mathrm{p}(\mathrm{L})+\mathrm{p}(\mathrm{M})) \\
& B_{H}=H \cdot p(L)+0 \cdot p(M)+0 \cdot p(H)=H \cdot p(L)
\end{aligned}
$$

Tabla 12. Cálculo del beneficio esperado.

Donde:

$B_{L}$ : beneficio esperado al demandar L.

$B_{M}$ : beneficio esperado al demandar $\mathrm{M}$.

$B_{H}$ : beneficio esperado al demandar $\mathrm{H}$.

$p(L)$ : estimación de la probabilidad de que el oponente actual demande L.

$p(M)$ : estimación de la probabilidad de que el oponente actual demande $\mathrm{M}$.

$p(H)$ : estimación de la probabilidad de que el oponente actual demande $\mathrm{H}$.

La estimación de la probabilidad de que el oponente demande L, M, H es el número relativo de elementos iguales a $\mathrm{L}, \mathrm{M}$ o $\mathrm{H}$ (respectivamente) en la memoria del agente. Es decir: 


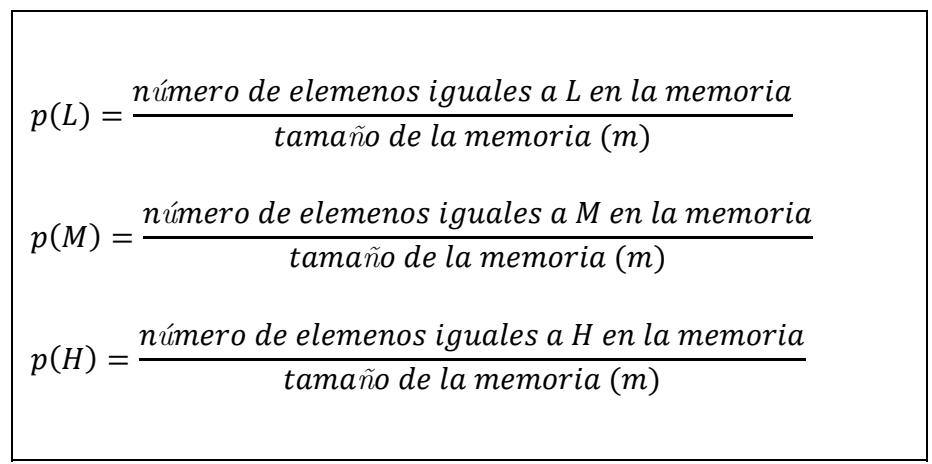

Tabla 13. Estimación de la probabilidad de que el oponente actual demande L, M o H.

Finalmente, los agentes escogen, de entre los tres valores calculados, aquél que maximiza el beneficio medio:

$$
\text { elección }=\max \left\{B_{L}, B_{M}, B_{H}\right\}
$$

Tabla 14. Elección de la estrategia que maximiza el beneficio de los agentes.

El modelo de AEY tiene dos versiones. En una primera versión del modelo, el llamado modelo $\sin \operatorname{tags}^{28}$, los agentes son indistinguibles entre sí, de tal manera que lo único que les diferencia es el contenido de sus memorias, el cual viene condicionado por la experiencia pasada de cada agente.

En una segunda versión, denominada modelo con tags $^{29}$, se consideran dos tipos de agentes. Para ello, se asigna a cada agente una característica diferenciadora (tag). Los agentes son capaces de identificar la tag del agente con el que interactúan en cada encuentro, lo cual les permite tomar decisiones distintas en función de la tag del agente con el que son emparejados en cada ronda. A pesar de que a priori la tag no tiene ningún significado económico intrínseco (puede representar la pertenencia a cierto grupo social, raza, sexo, o simplemente el color de ojos), Axtell, Epstein y Young (2000) demuestran que, simplemente por el hecho de introducir esta tag, pueden emerger diversas normas discriminatorias en el sistema, situación que no ocurría en el modelo sin tags.

${ }^{28}$ Model with one agent type.

${ }^{29}$ Tag model. 


\subsubsection{RESULTADOS}

\subsubsection{MODELO SIN TAGS}

Se parte de una población de $N$ agentes $^{30}$ que son emparejados al azar para jugar al juego de la demanda de Nash. Cada uno de ellos dispone de una memoria de tamaño $m$ donde almacenarán las decisiones tomadas por sus oponentes en las últimas $m$ rondas, tal como se indica de manera esquemática en la Figura 25.

\section{MEMORIA}
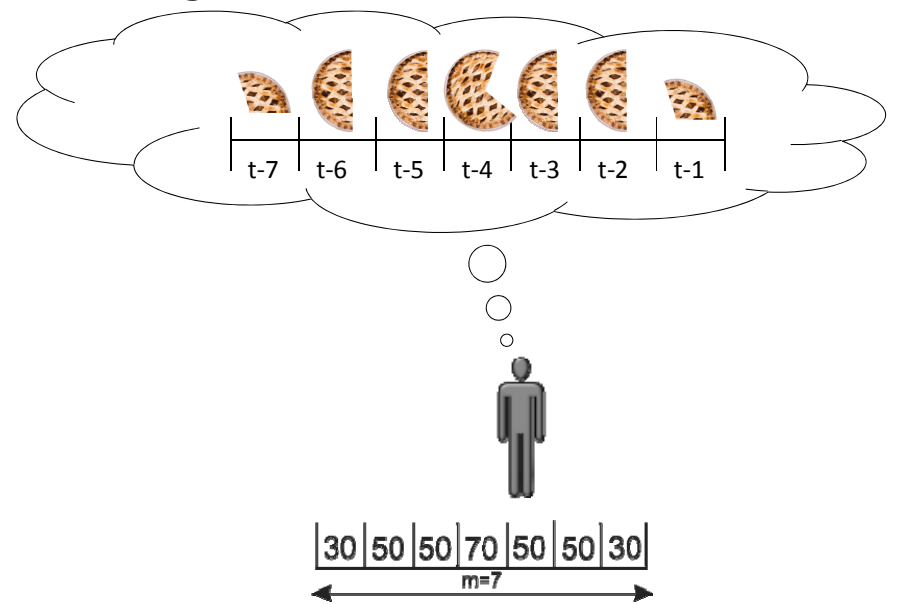

Figura 25. Representación esquemática del uso de la memoria de un agente, con un tamaño de memoria igual a 7 .

Cada agente, en función del contenido de su memoria, demandará cierta cantidad de la "tarta" con el objetivo de maximizar su pago, utilizando para ello las ecuaciones de la Tabla 12 .

No obstante, con cierta probabilidad (definida por el parámetro del modelo $\varepsilon$ ), los agentes escogen una de las tres posibles demandas al azar, de manera equiprobable. Esto representa el hecho de que, en ocasiones, los seres humanos toman decisiones que no son

${ }^{30} N$ es un número par. 
necesariamente las más racionales, con el objetivo de explorar e intentar obtener así un beneficio mayor.

En resumen, los agentes ofrecen una respuesta ruidosa: con una probabilidad relativamente grande (1- 1 ) los agentes escogen la estrategia que maximiza su beneficio (según la Tabla 12) y con una probabilidad relativamente pequeña $(\varepsilon)$, escogen una estrategia al azar con la misma probabilidad.

Para conocer el contenido de la memoria de los agentes y permitir así una visualización gráfica del régimen en el que se encuentra el sistema en cada momento, se recurre a la representación de los agentes en un simplex. En él, los agentes se representan proporcionalmente más cerca de los vértices L, M o $\mathrm{H}$ en función del contenido de su memoria. La Figura 26 muestra la apariencia del simplex en el instante en el que se crean los agentes, antes de que comiencen las interacciones entre los mismos, momento en el que el contenido de su memoria es aleatorio. Como se observa en dicha figura, el simplex se encuentra dividido en tres regiones. Cada una de ellas indica la estrategia que seguirán los agentes que se encuentran sobre ella (con probabilidad 1- $\varepsilon$ ) en la siguiente interacción con otro agente.

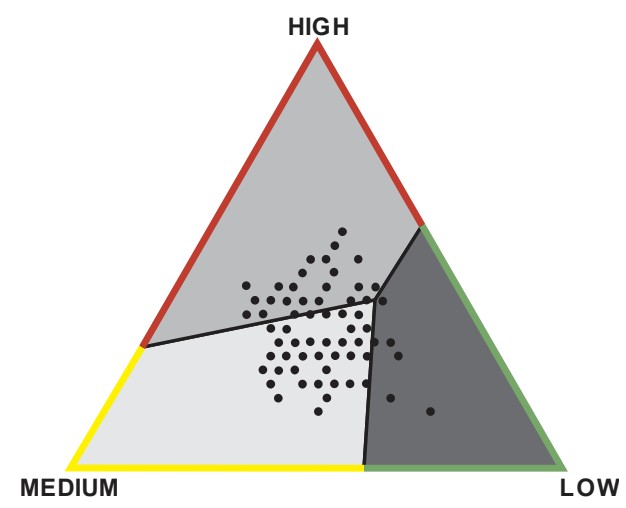

Figura 26. Modelo de AEY sin tags. Apariencia del simplex antes de comenzar la simulación.

El contenido inicial de la memoria de los agentes es aleatorio. Tras una serie de interacciones entre los agentes, pueden alcanzarse dos regímenes en el sistema: 
- Equilibrio paritario (o equilibrio equitativo ${ }^{31}$ ): Se produce un reparto paritario: todos los agentes se acaban coordinando y demandan y reciben $\mathrm{M}$. En este caso se alcanza un equilibrio de Nash en el que el excedente, además de repartirse de manera equitativa, lo hace de manera eficiente: dado que la suma de las dos demandas de los agentes implicados en el juego es igual a la unidad, no queda ninguna porción de recurso sin repartir entre los agentes. El simplex que se muestra en la Figura 27 representa el contenido de la memoria de los agentes cuando se ha alcanzado el equilibrio paritario en el sistema.

- Estado fluctuante ${ }^{32}$ : En este caso el reparto no es ni paritario ni eficiente: los agentes no se coordinan y demandan en ocasiones $\mathrm{L}$ y en ocasiones $\mathrm{H}$, de tal manera que el sistema no alcanza ningún equilibrio de Nash. Los agentes que demandan $\mathrm{L}$ reciben siempre $\mathrm{L}$, y los agentes que demandan $\mathrm{H}$ reciben $\mathrm{H}$ o cero en función de la demanda de su oponente. Se trata, por tanto, de un reparto ineficiente, ya que en ocasiones queda cierta cantidad de recurso sin repartir. El simplex de la Figura 28 representa el contenido de la memoria de los agentes cuando el sistema se encuentra en un estado fluctuante.

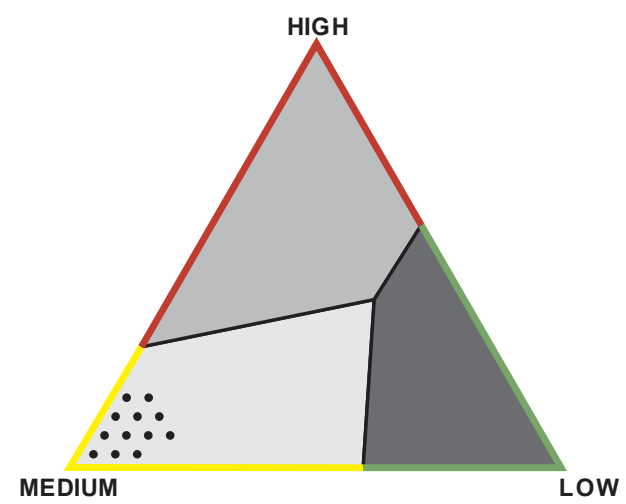

Figura 27. Equilibrio paritario. $\mathrm{N}=100, \varepsilon=0.2$, $\mathrm{m}=30$

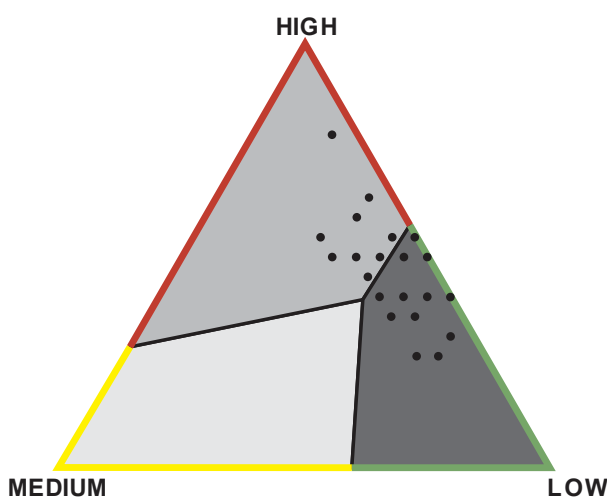

Figura 28. Estado fluctuante. $\mathrm{N}=100, \varepsilon=0.2$, $\mathrm{m}=30$

Las condiciones de parada de la simulación, según se alcance uno u otro régimen, se indican en la Tabla 15.

\footnotetext{
${ }^{31}$ Equitable equilibrium

${ }^{32}$ Fractious state
} 
- Equilibrio paritario: Se considera que el sistema ha alcanzado el equilibrio paritario si todos los agentes tienen, al menos, $(1-\varepsilon) \cdot \mathrm{m}$ posiciones de memoria iguales a $\mathrm{M}$.

- Estado fluctuante: Se considera que el sistema ha alcanzado el estado fluctuante si todos los agentes tienen, como máximo, $\varepsilon \cdot \mathrm{m}$ posiciones de memoria iguales a $\mathrm{M}$.

Tabla 15. Condiciones de parada de la simulación en el modelo sin tags.

Por consiguiente, el modelo de AEY sin tags presenta dos estados persistentes: el equilibrio paritario y el estado fluctuante. Si la respuesta de los agentes no fuera ruidosa (es decir, si los agentes nunca recurriesen a la exploración, lo cual se conseguiría haciendo que $\varepsilon=0$ ), el sistema quedaría atrapado en uno de estos estados ${ }^{33}$. Sin embargo, la presencia de ruido en el sistema $(\varepsilon \neq 0)$, hace que el sistema sea ergódico. Esto significa que el sistema puede migrar de uno a otro estado a otro si se la da el tiempo suficiente, no quedando atrapado en ninguno de los dos estados. Como consecuencia, el estado en el que se encuentra el sistema en un momento indeterminado se hace independiente de las condiciones iniciales. No obstante, Axtell, Epstein y Young demostraron que, si se permite que el sistema continúe durante un tiempo indefinidamente largo, éste permanecerá más tiempo en el equilibrio paritario que en el estado fluctuante. Es decir, el equilibrio equitativo es el único estado estocásticamente estable que puede alcanzar el sistema en el modelo sin tags.

En lo sucesivo, hablaremos de modelo perturbado cuando se permitan respuestas ruidosas (es decir, cuando $\varepsilon \neq 0$ ) y de modelo no-perturbado cuando no se permitan (es decir, cuando $\varepsilon=0$ ).

En cualquier caso, el tiempo de permanencia del sistema en cada uno de los estados persistentes depende de ciertos parámetros del modelo (principalmente del número de agentes $N$ y del tamaño de la memoria $m$ ). Axtell, Epstein y Young definieron el tiempo de transición como el tiempo que el sistema tarda en alcanzar el equilibrio paritario partiendo del estado fluctuante. La Figura 29 representa el tiempo de

${ }^{33}$ Si no hay ruido en el sistema $(\varepsilon=0)$, tanto el equilibrio paritario como el estado fluctuante se convierten en estados absorbentes. 
transición en función de $\mathrm{m}$, para distintos valores de $\varepsilon$. Dicho experimento fue llevado a cabo a partir de nuestra réplica del modelo de AEY.

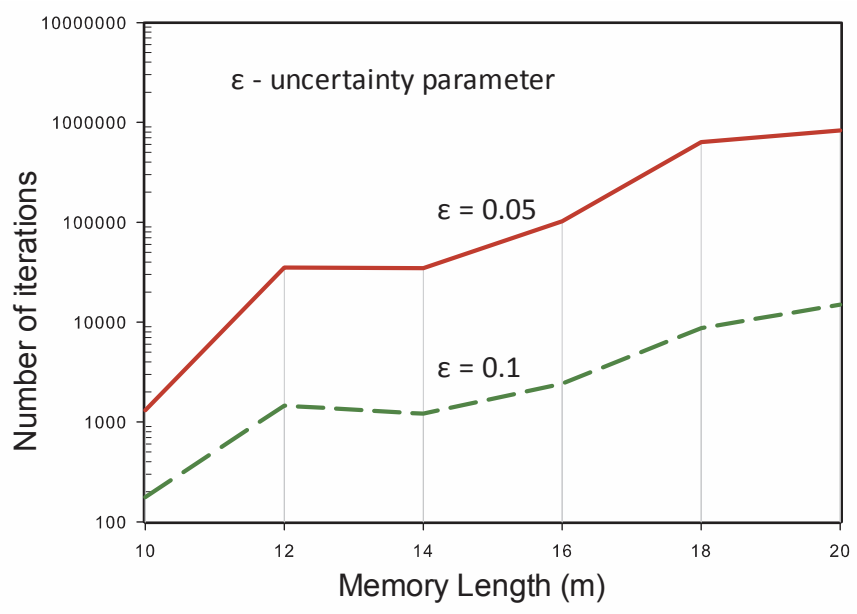

Figura 29. Tiempo de transición en función del tamaño de memoria $(m)$, para distintos valores de $\varepsilon$. Número de agentes $N=10$.

Axtell, Epstein y Young comprobaron que, para valores altos de $N$ y $m$, el tiempo de transición se hacía infinitamente largo. Esto se traduce en que, en la práctica, para valores elevados de $N$ y de $m$, el sistema queda atrapado en uno de los dos estados persistentes. Esto es lo que Axtell, Epstein y Young denominaron ergodicidad rota. No obstante, debe tenerse en cuenta que, en una situación real, la interacción entre agentes no se lleva a cabo mediante emparejamientos aleatorios, como ocurre en el modelo de AEY, sino que los agentes forman parte de una red social que determina el número de agentes con los que cada agente interactúa. Este número de agentes es inferior al necesario para que se produzca la ruptura de la ergodicidad del sistema, lo cual hace que el tiempo de transición se reduzca. 


\subsubsection{MODELO CON TAGS}

Se parte, al igual que en el modelo sin tags, de una población de $N$ agentes, sólo que en esta ocasión la mitad de la población (N/2) tendrá una tag y la otra mitad tendrá otra tag. Por simplicidad, el valor que se le asigna a la tag es el color del agente, por lo que la mitad de la población será de un color, y la otra mitad de otro color.

En el modelo con tags, cada agente está dotado de dos memorias. En una de ellas (denominada memoria intratype) se almacenan las decisiones tomadas por agentes de su misma tag, mientras que en otra memoria (llamada memoria intertype) se almacenan las decisiones tomadas por los agentes que tienen distinta tag, siguiendo el esquema de la Figura 30.

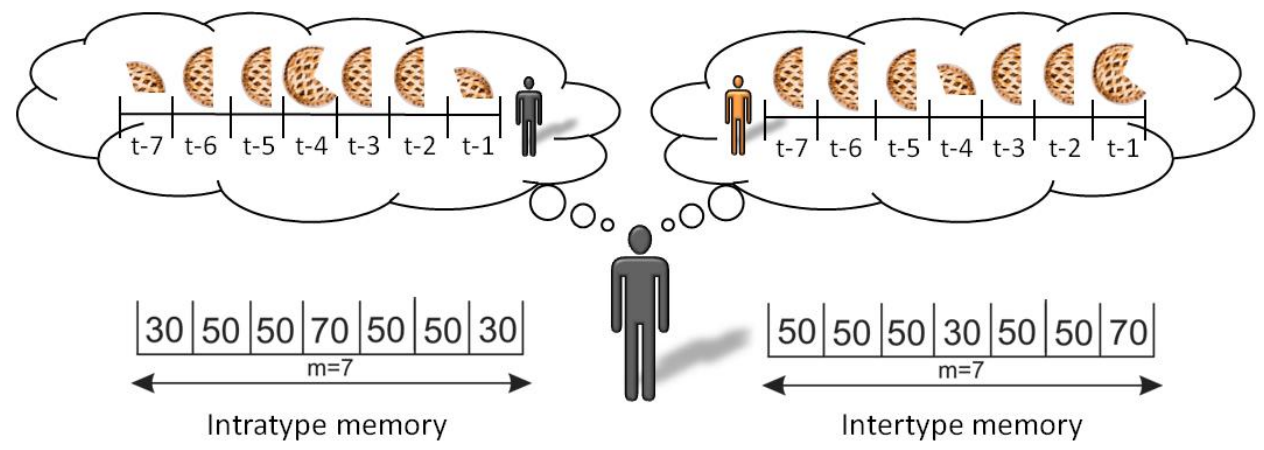

Figura 30. Representación esquemática de las memorias intratype e intertype de un agente, con un tamaño de memoria igual a 7 .

Al igual que en el modelo sin tags, con probabilidad (1- $\varepsilon$ ), los agentes utilizarán el contenido de su memoria (intratype o intertype, en función de la tag del agente contra el que se enfrenten en cada ronda) para tratar de maximizar su beneficio; mientras que con probabilidad $\varepsilon$, escogerán una estrategia al azar (L, M o H con equiprobabilidad).

Puesto que en el Modelo de Clases cada agente dispone de dos memorias, serán necesarios dos simplex para representar el contenido de las mismas: uno para representar los juegos entre agentes con la misma tag (juegos intratype) y otro para representar los juegos entre agentes de distinta tag (juegos intertype). 


\section{JUEGOS INTRATYPE (interacciones entre agentes con la misma tag)}

En el caso de las interacciones intratype, el sistema puede alcanzar uno de los siguientes estados:

- Equilibrio paritario (Figura 31a): Todos agentes acaban demandando M (reparto paritario) independientemente de su tag.

- Estado fluctuante (Figura 31b): Independientemente de su tag, los agentes demandan unas veces $\mathrm{L}$ y otras veces $\mathrm{H}$ sin que se alcance ningún equilibrio en el sistema.

- Segregación intratype (Figura 31c): Los agentes alcanzan regímenes distintos en función de cuál sea su tag. En el caso que se muestra en la figura, todos los agentes de color negro alcanzan un equilibrio paritario y todos los agentes de color naranja alcanzan el estado fluctuante.

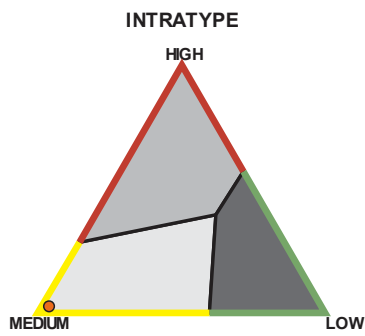

(a)

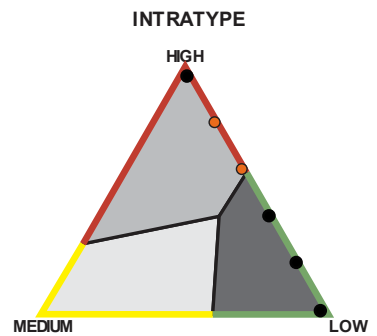

(b)

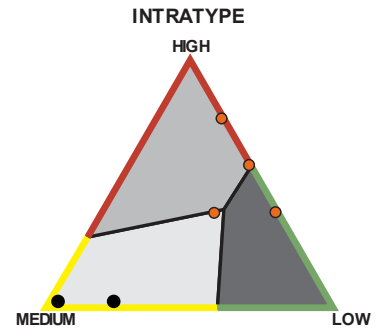

(c)

Figura 31. Los tres posibles resultados que pueden darse en las interacciones intratype.

\section{JUEGOS INTERTYPE (interacciones entre agentes de distinta tag)}

Cuando las interacciones se producen entre agentes de distinta tag, el sistema puede alcanzar uno de los siguientes estados:

- Equilibrio paritario (Figura 32a): Todos agentes acaban demandando M (reparto paritario) independientemente de su tag.

- Equilibrio segregado / segregación intertype (Figura 32b): El sistema alcanza un equilibrio de Nash en el que todos los agentes de una misma tag demandan L y todos los agentes de la otra tag demandan $\mathrm{H}$. El reparto, a pesar 
de ser discriminatorio, es eficiente, ya que se reparte todo el excedente entre los agentes.

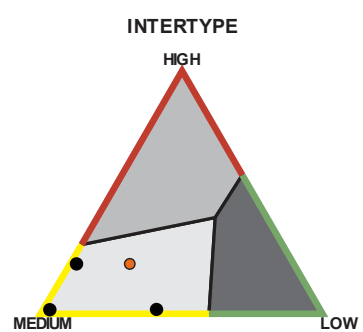

(a)

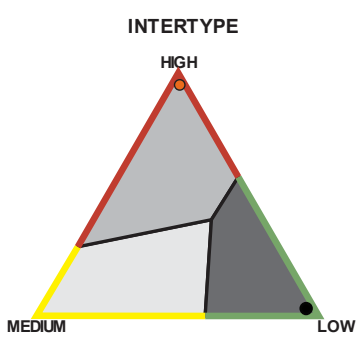

(b)

Figura 32. Los dos posibles resultados que pueden darse en las interacciones intertype.

En ambos tipos de juego (intratype e intertype), los criterios de parada considerados son los que se muestran en la Tabla 16.

- Equilibrio paritario (EQ): Todos los agentes tienen, al menos, $(1-\varepsilon) \cdot \mathrm{m}$ posiciones de memoria iguales a $\mathrm{M}$.

- Equilibrio segregado (IQ): Todos los agentes tienen, cómo máximo $\varepsilon \cdot \mathrm{m}$ posiciones de memoria iguales a $\mathrm{M}, \mathrm{y}$, además, un grupo de agentes tiene $(1-\varepsilon) \cdot \mathrm{m}$ instancias de $\mathrm{L}$ en su memoria y el resto de agentes tiene $(1-\varepsilon) \cdot \mathrm{m}$ instancias de $\mathrm{H}$ en su memoria

- Estado fluctuante (FR): Todos los agentes tienen, como mínimo, una combinación de instancias de $\mathrm{L}$ y $\mathrm{H}$ igual a $(1-\varepsilon) \cdot \mathrm{m}$.

Tabla 16. Criterios de parada de la simulación en el modelo con tags.

Los resultados que se muestran en la Figura 31c y en la Figura 32b son los más destacables del modelo de AEY, ya que demuestran que, solamente el hecho de introducir una característica sin significado económico (la tag, en este caso el color del agente), puede provocar que emerja segregación entre los agentes. 


\subsection{ANÁLISIS DE SENSIBILIDAD E INFLUENCIA DE CONDICIONES INICIALES. NUEVA REGLA DE DECISIÓN}

Una vez presentados el modelo de AEY y los experimentos llevados a cabo con nuestra réplica del modelo en la sección anterior, se mostrarán a continuación una serie de extensiones al modelo original realizadas sobre nuestra réplica del modelo.

El primer paso consistió en realizar un análisis de sensibilidad de la matriz de pagos para analizar cómo afectaban a los resultados los valores asociados a L, M y H (sección 3.3.1). En segundo lugar, se modificaron las condiciones iniciales con las que partían los agentes, modificando para ello la configuración de sus memorias en el momento de su creación (sección 3.3.2). Finalmente, se sustituyó la regla de decisión original del AEY por una nueva regla de decisión que requería menos habilidades cognoscitivas por parte de los agentes, y se analizó el impacto de esta nueva regla de decisión tanto en los regímenes que se alcanzan en el sistema como en la frecuencia con la que se alcanzan cada uno de ellos (sección 3.3.3). Las extensiones que se presentan en esta sección se encuentran publicadas en (Poza et al., 2011).

\subsubsection{ANÁLISIS DE SENSIBILIDAD}

En los primeros experimentos realizados con nuestra réplica se respetó la matriz de pagos del modelo de AEY, pues el objetivo de la réplica era la verificación del modelo original. Una vez concluida la fase de verificación, se procedió a comprobar cómo variaban los resultados del modelo original al cambiar los valores de la matriz de pagos.

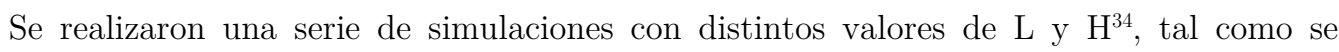
indica en la Tabla 17.

${ }^{34}$ Para todo L y $\mathrm{H}$, siempre se cumple que $\mathrm{L}+\mathrm{H}=100 \%$. 


\begin{tabular}{cr|c|c|}
\multirow{2}{*}{$\mathrm{P} 1 \backslash \mathrm{P} 2$} & \multicolumn{1}{c}{$\mathrm{H}$} & \multicolumn{1}{c}{$\mathrm{M}$} & \multicolumn{1}{c}{$\mathrm{L}$} \\
\cline { 2 - 4 } $\mathrm{H}$ & $0 \backslash 0$ & $0 \backslash 0$ & $\mathbf{9 5} \backslash \mathbf{5}$ \\
\cline { 2 - 4 } $\mathrm{M}$ & $0 \backslash 0$ & $\mathbf{5 0} \backslash \mathbf{5 0}$ & $50 \backslash 5$ \\
\cline { 2 - 4 } $\mathrm{L}$ & $\mathbf{5} \backslash \mathbf{9 5}$ & $5 \backslash 50$ & $5 \backslash 5$ \\
\cline { 2 - 4 } & & &
\end{tabular}

\begin{tabular}{|c|c|c|c|}
\hline $\mathrm{P} 1 \backslash \mathrm{P} 2$ & $\mathrm{H}$ & M & L \\
\hline $\mathrm{H}$ & $0 \backslash 0$ & $0 \backslash 0$ & $80 \backslash 20$ \\
\hline M & $0 \backslash 0$ & $50 \backslash 50$ & $50 \backslash 20$ \\
\hline $\mathrm{L}$ & $20 \backslash 80$ & $20 \backslash 50$ & $20 \backslash 20$ \\
\hline
\end{tabular}

\begin{tabular}{c|c|c|c|} 
P1 & \multicolumn{1}{c}{$\mathrm{H}$} & \multicolumn{1}{c}{$\mathrm{M}$} & \multicolumn{1}{c}{$\mathrm{L}$} \\
\cline { 2 - 4 } $\mathrm{H}$ & $0 \backslash 0$ & $0 \backslash 0$ & $\mathbf{6 5} \backslash \mathbf{3 5}$ \\
\cline { 2 - 4 } $\mathrm{M}$ & $0 \backslash 0$ & $\mathbf{5 0} \backslash \mathbf{5 0}$ & $50 \backslash 35$ \\
\cline { 2 - 4 } $\mathrm{L}$ & $\mathbf{3 5} \backslash \mathbf{6 5}$ & $35 \backslash 50$ & $35 \backslash 35$ \\
\cline { 2 - 4 } & &
\end{tabular}

\begin{tabular}{cc|c|c|} 
P1P2 & \multicolumn{1}{c}{$\mathrm{H}$} & $\mathrm{M}$ & $\mathrm{L}$ \\
\cline { 2 - 4 } $\mathrm{H}$ & $0 \backslash 0$ & $0 \backslash 0$ & $\mathbf{9 0} \backslash \mathbf{1 0}$ \\
\cline { 2 - 4 } $\mathrm{M}$ & $0 \backslash 0$ & $\mathbf{5 0} \backslash \mathbf{5 0}$ & $50 \backslash 10$ \\
\cline { 2 - 4 } $\mathrm{L}$ & $\mathbf{1 0} \backslash \mathbf{9 0}$ & $10 \backslash 50$ & $10 \backslash 10$ \\
\cline { 2 - 4 } $\mathrm{P} 1 \mathrm{P} 2$ & $\mathrm{H}$ & $\mathrm{M}$ & $\mathrm{L}$ \\
$\mathrm{H}$ & $0 \backslash 0$ & $0 \backslash 0$ & $\mathbf{7 5} \backslash \mathbf{2 5}$ \\
\cline { 2 - 4 } $\mathrm{M}$ & $0 \backslash 0$ & $\mathbf{5 0} \backslash \mathbf{5 0}$ & $50 \backslash 25$ \\
\cline { 2 - 4 } $\mathrm{L}$ & $\mathbf{2 5} \backslash \mathbf{7 5}$ & $25 \backslash 50$ & $25 \backslash 25$ \\
\cline { 2 - 4 } & &
\end{tabular}

\begin{tabular}{c|c|c|c|}
\multirow{3}{*}{$\mathrm{P} 1 \mathrm{P} 2$} & \multicolumn{1}{c}{$\mathrm{H}$} & $\mathrm{M}$ & $\mathrm{L}$ \\
\cline { 2 - 4 } $\mathrm{H}$ & $0 \backslash 0$ & $0 \backslash 0$ & $\mathbf{6 0} \backslash \mathbf{4 0}$ \\
\cline { 2 - 4 } $\mathrm{M}$ & $0 \backslash 0$ & $\mathbf{5 0} \backslash \mathbf{5 0}$ & $50 \backslash 40$ \\
\cline { 2 - 4 } $\mathrm{L}$ & $\mathbf{4 0} \backslash \mathbf{6 0}$ & $40 \backslash 50$ & $40 \backslash 40$ \\
\cline { 2 - 4 } & &
\end{tabular}

\begin{tabular}{c|c|c|c|}
\multirow{2}{*}{$\mathrm{P} 1 \backslash \mathrm{P} 2$} & $\mathrm{H}$ & \multicolumn{1}{c}{$\mathrm{M}$} & \multicolumn{1}{c}{$\mathrm{L}$} \\
\cline { 2 - 4 } $\mathrm{H}$ & $0 \backslash 0$ & $0 \backslash 0$ & $\mathbf{8 5} \backslash \mathbf{1 5}$ \\
\cline { 2 - 4 } $\mathrm{M}$ & $0 \backslash 0$ & $\mathbf{5 0} \backslash \mathbf{5 0}$ & $50 \backslash 15$ \\
\cline { 2 - 4 } $\mathrm{L}$ & $\mathbf{1 5} \backslash \mathbf{8 5}$ & $15 \backslash 50$ & $15 \backslash 15$ \\
\cline { 2 - 4 } & & &
\end{tabular}

\begin{tabular}{c|c|c|c|} 
& \multicolumn{1}{c}{$\mathrm{H} 1 \backslash \mathrm{P} 2$} & \multicolumn{1}{c}{$\mathrm{M}$} & $\mathrm{L}$ \\
\cline { 2 - 4 } $\mathrm{H}$ & $0 \backslash 0$ & $0 \backslash 0$ & $\mathbf{7 0} \backslash \mathbf{3 0}$ \\
\cline { 2 - 4 } $\mathrm{M}$ & $0 \backslash 0$ & $\mathbf{5 0} \backslash \mathbf{5 0}$ & $50 \backslash 30$ \\
\cline { 2 - 4 } $\mathrm{L}$ & $\mathbf{3 0} \backslash \mathbf{7 0}$ & $30 \backslash 50$ & $30 \backslash 30$ \\
\cline { 2 - 4 } & &
\end{tabular}

\begin{tabular}{c|c|c|c|} 
P1\P2 & \multicolumn{1}{c}{$\mathrm{H}$} & \multicolumn{1}{c}{$\mathrm{M}$} & $\mathrm{L}$ \\
\cline { 2 - 4 } $\mathrm{H}$ & $0 \backslash 0$ & $0 \backslash 0$ & $\mathbf{5 5} \backslash \mathbf{4 5}$ \\
\cline { 2 - 4 } $\mathrm{M}$ & $0 \backslash 0$ & $\mathbf{5 0} \backslash \mathbf{5 0}$ & $50 \backslash 45$ \\
\cline { 2 - 4 } $\mathrm{L}$ & $\mathbf{4 5} \backslash \mathbf{5 5}$ & $45 \backslash 50$ & $45 \backslash 45$ \\
\cline { 2 - 4 } & & &
\end{tabular}

Tabla 17. Matrices de pagos empleadas en el análisis de sensibilidad.

Se midió el tiempo de transición del sistema (es decir, el tiempo que tarda el sistema en alcanzar el equilibrio paritario partiendo del estado segregado) empleando distintas matrices de pagos. La Figura 33 muestra el tiempo de transición para tres valores de L seleccionados.

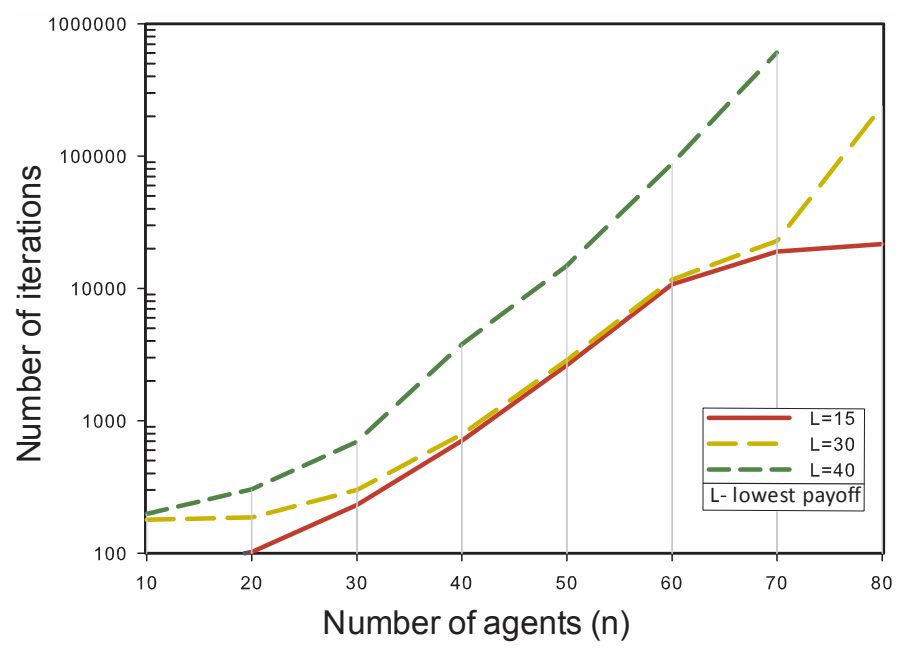

Figura 33. Tiempo de transición en función del número de agentes para distintas matrices de pagos. Tamaño de memoria $\mathrm{m}=10$. Parámetro de ruido $\varepsilon=0.1$. Se representa el valor promedio calculado a partir de 1.000 repeticiones de la simulación para cada par de valores $(n, L)$.

Se comprobó que cuanto mayor era el valor asignado a L (y, por tanto, menor el valor asociado a H), mayor era el tiempo de transición. La razón es la siguiente: el pago que recibe un agente cuando demanda $L$ siempre es $\mathrm{L}$, independientemente de la demanda de su oponente (ver Tabla 12). Cuando L toma valores altos, los agentes no tienen incentivos para demandar $\mathrm{M} \mathrm{o} \mathrm{H}$, pues el beneficio esperado de estas opciones es inferior. 
Sin embargo, mientras que el beneficio esperado al escoger L es fijo, el beneficio de escoger $\mathrm{M}$ o $\mathrm{H}$ depende del contenido de la memoria del agente $\mathrm{y}$, por tanto, de las decisiones de sus oponentes en encuentros anteriores. Por esta razón, aunque durante las primeras etapas de la simulación los agentes tienden a escoger L, según avanza la simulación, los agentes comienzan a observar que sus oponentes han demandado L, lo cual hace que el beneficio esperado de escoger $M$ supere al de escoger $L$ y finalmente se alcance el equilibrio paritario.

Nótese que las fronteras de decisión del simplex se van afectadas al variar la matriz de pagos. En la Figura 34 se muestra el aspecto que ofrece el simplex en el momento en el que el contenido de la memoria de los agentes es aleatorio cuando el valor asignado a $\mathrm{L}$ es 40\%. Al compararlo con el simplex de la Figura 26 se aprecia que, en este caso, la zona de mayor área corresponde a la región donde el mayor beneficio se obtiene al demandar L, y, además, la mayor parte de los agentes se sitúa inicialmente sobre esta zona, lo cual explica por qué los agentes tienden a demandar L durante las primeras etapas de la iteración, antes de alcanzar el equilibrio paritario, lo cual hace que el tiempo de transición aumente si lo comparamos con los experimentos en los que se emplea la matriz de pagos original.

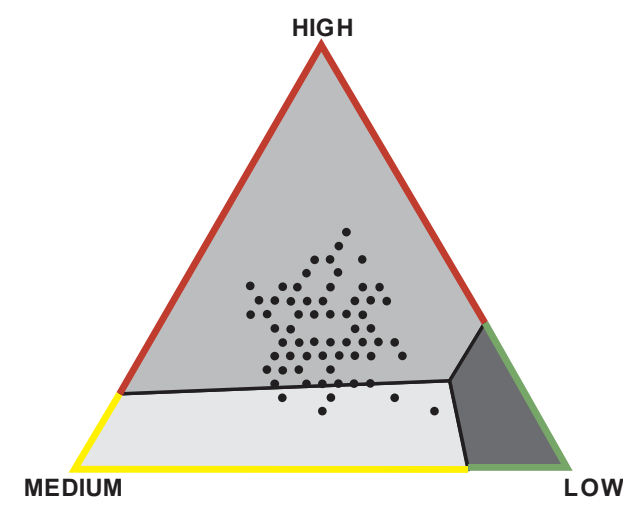

Figura 34. Apariencia del simplex cuando el valor asignado a L es 40\%, en las mismas condiciones que el simplex de la Figura 26. 


\subsubsection{CONDICIONES INICIALES}

En el modelo de AEY, las memorias de los agentes eran inicializadas con $m$ valores de L, M y H tomados al azar y de manera equiprobable. La siguiente modificación que se llevó a cabo consistió en modificar la estructura de la memoria de los agentes durante las primeras etapas de la simulación. Concretamente, los agentes fueron inicializados con una única posición de memoria, en la que se almacenaba un valor aleatorio (L, M o H con la misma probabilidad). En la primera interacción, los agentes utilizan el único valor que contiene su memoria para elegir la estrategia que maximiza su beneficio, según la Tabla 12. Tras esta primera iteración, el agente añade a su memoria la decisión de su oponente actual, aumentando así la memoria en una unidad. En la siguiente iteración, los agentes calculan el beneficio esperado a partir de la información de las dos celdas de memoria que tienen en este momento. Tras esta segunda iteración, aumentan el tamaño de la memoria en una unidad para almacenar la demanda de su oponente actual. En interacciones sucesivas, el tamaño de la memoria continúa aumentando en una unidad por interacción hasta que ésta alcanza el tamaño $m$, momento a partir del cual el tamaño de la memoria se mantiene constante en las sucesivas interacciones.

Nuestros experimentos nos permitieron comprobar cómo este cambio aparentemente pequeño en las condiciones iniciales del sistema afectaba considerablemente al resultado de las simulaciones. Como puede observarse en la Figura 35, el tiempo necesario para que el sistema alcanzase el equilibrio paritario aumentó significativamente tras la modificación de las condiciones iniciales del sistema. 


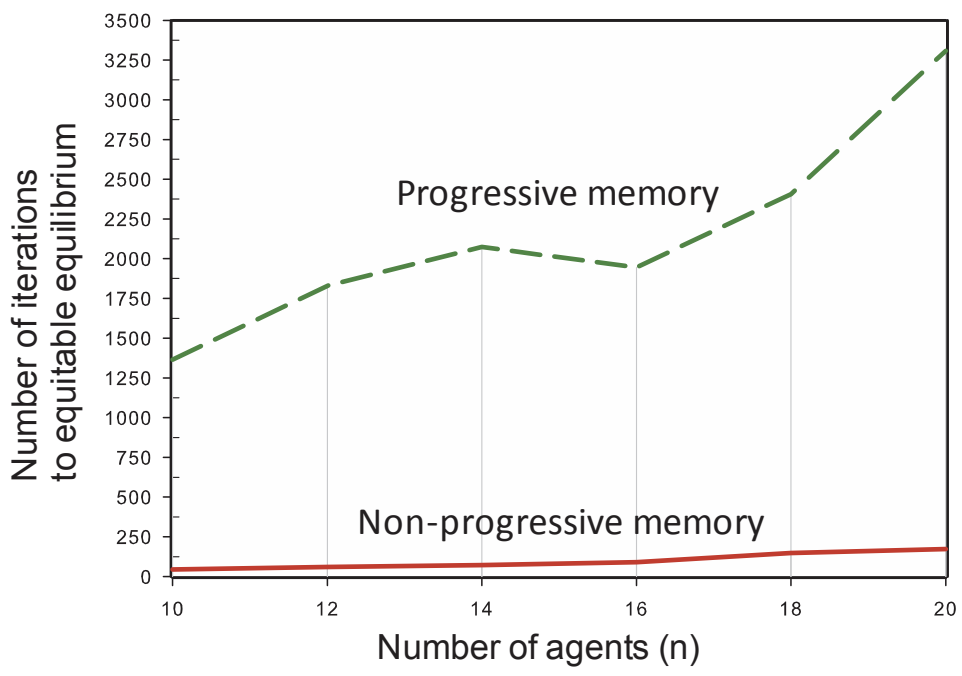

Figura 35. Número de rondas hasta alcanzar el equilibrio paritario, partiendo de condiciones iniciales aleatorias. Comparación entre memoria no-progresiva (modelo de AEY) y memoria progresiva (réplica del modelo de AEY modificada). Se representa el valor promedio calculado a partir de 100 repeticiones de la simulación para cada valor de $\mathrm{n}$. Parámetro de ruido $\varepsilon=0.2$. Tamaño de memoria $\mathrm{m}=12$.

La razón de este comportamiento se encuentra en que, inicialmente, la probabilidad de que la memoria de un agente contenga $\mathrm{L}$ o $\mathrm{H}$ es el doble de la probabilidad de que ésta contenga M. Esto hace que durante las primeras etapas de la simulación, el sistema se aproxime a un estado fluctuante. No obstante, la presencia de ruido en el sistema hace que finalmente éste alcance el equilibrio paritario. Debido a esta fase transitoria en la que el sistema se aproxima a un estado fluctuante, el tiempo que el sistema tarda en alcanzar el equilibrio paritario es superior al del modelo de AEY, tal como se puede comprobar en la Figura 35.

\subsubsection{NUEVA REGLA DE DECISIÓN}

En nuestra siguiente extensión del modelo, se sustituyó la regla de decisión que empleaban los agentes en el modelo original por otra que requería menos habilidades cognoscitivas. La razón para efectuar esta modificación radica en que numerosos estudios 
de Economía Experimental demuestran que los seres humanos no tienden a consumir un tiempo excesivo en la realización de cómputos para tomar decisiones (Epstein, 2001).

La nueva regla de decisión, que requiere una capacidad de cómputo mucho inferior que la del modelo de AEY, consiste en lo siguiente: los agentes, observando el contenido de su memoria, estiman la probabilidad de que su oponente demande L, M o H de la misma manera que en el modelo de AEY (Tabla 13). A continuación, en lugar de computar el beneficio que obtendrían para cada una de las posibles estrategias (como se hace en el modelo de AEY, Tabla 12), simplemente eligen la mejor respuesta ante la demanda más probable del oponente, considerada como la $\underline{\text { moda }}$ del contenido actual de su memoria. Es decir: si la demanda más probable del oponente es L, el agente erigirá H; si la demanda más probable del oponente es M, el agente elegirá M; y si la demanda más probable del oponente es H, el agente elegirá L (ver Tabla 18).

$$
\begin{aligned}
& \text { si } \max \{p(L), P(M), p(H)\}=p(L) \rightarrow \text { elección }=H \\
& \text { si } \max \{p(L), P(M), p(H)\}=p(M) \rightarrow \text { elección }=M \\
& \text { si } \max \{p(L), P(M), p(H)\}=p(H) \rightarrow \text { elección }=L
\end{aligned}
$$

Tabla 18. Elección de la mejor estrategia ante la decisión más probable del oponente.

Donde:

$p(L)$ : estimación de la probabilidad de que el oponente actual demande L.

$p(M)$ : estimación de la probabilidad de que el oponente actual demande M.

$p(H)$ : estimación de la probabilidad de que el oponente actual demande $\mathrm{H}$.

Al igual que en el modelo de AEY, existe cierta posibilidad de exploración (definida por el parámetro $\varepsilon$ ) mediante la cual los agentes escogen $\mathrm{L}, \mathrm{M}$ o $\mathrm{H}$ con la misma probabilidad.

Tras la modificación de la regla de decisión de los agentes, cabe plantearse dos cuestiones: la primera es si se alcanzarán los mismos regímenes que en el modelo de AEY. Suponiendo que la respuesta a la pregunta sea afirmativa, la segunda cuestión que cabría preguntarse sería: ¿afecta la introducción de la nueva regla de decisión a la frecuencia con la que se alcanzan tales regímenes? 


\subsubsection{1. $\quad$ FOCOS DE ATRACCIÓN}

Se observó que los focos de atracción que alcanza el sistema son los mismos que se alcanzaban en el modelo original. Es decir, en el modelo sin tags, puede alcanzarse bien el equilibrio paritario (Figura 36a), bien el estado fluctuante (Figura 36b). Nótese cómo han variado las fronteras de decisión del simplex al modificar la regla de decisión (compárense con la Figura 27 y la Figura 28, respectivamente).

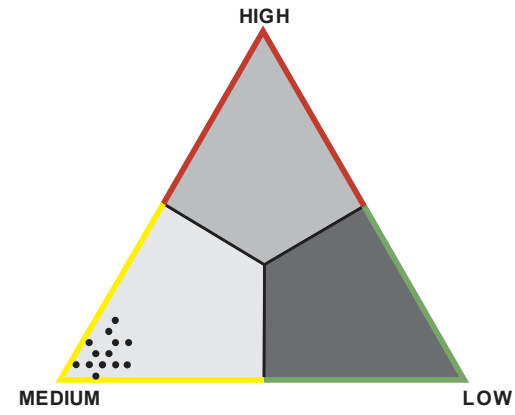

(a)

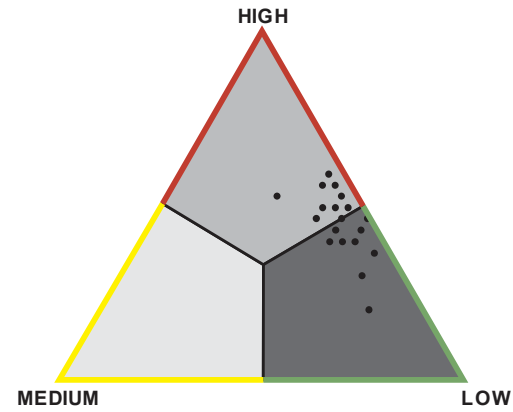

(b)

Figura 36. Posibles focos de atracción (equilibrio paritario y estado fluctuante) en el modelo sin tags tras la introducción de la nueva regla de decisión. $\mathrm{N}=100, \varepsilon=0.2, \mathrm{~m}=30$.

\subsubsection{VARIACIÓN EN LA FRECUENCIA CON LA QUE SE ALCANZAN LOS REGÍMENES}

A pesar de que con la nueva regla de decisión, el sistema alcanza los mismos regímenes que en el modelo de AEY, comprobamos mediante simulación que, mientras que con la regla de decisión original el sistema alcanzaba el equilibrio paritario en la mayoría de las ocasiones, con la nueva regla de decisión se favorecía la emergencia de estados fluctuantes.

Para extraer esta conclusión, inicializamos el sistema con condiciones aleatorias y contabilizamos el número de experimentos que alcanzaban el equilibrio paritario y el estado fluctuante. El experimento se repitió para varios valores del número de agentes $(N)$ y tamaños de memoria $(m)$. La Figura 37 muestra el porcentaje de experimentos que alcanzaron el estado paritario cuando se empleó la regla de decisión original, mientras que la Figura 38 corresponde a la nueva regla de decisión. Al comparar ambas figuras, puede observarse que, independientemente del número de agentes y del tamaño de la memoria, con la regla original resulta muy improbable que el sistema alcance un estado 
fluctuante, mientras que con la nueva regla de decisión, la emergencia de estados fluctuantes se hace más frecuente.

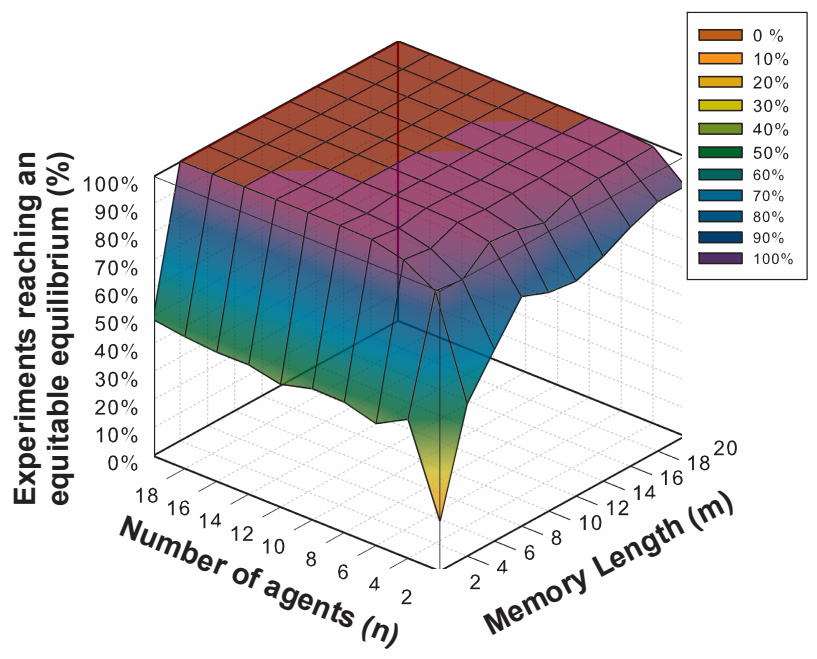

Figura 37. Porcentaje de experimentos que terminan en equilibrio paritario cuando se emplea la regla de decisión original. Se representa el valor promedio calculado a partir de 1.000 repeticiones de la simulación para cada par de valores (n, m). Parámetro de ruido $\varepsilon=0.2$.

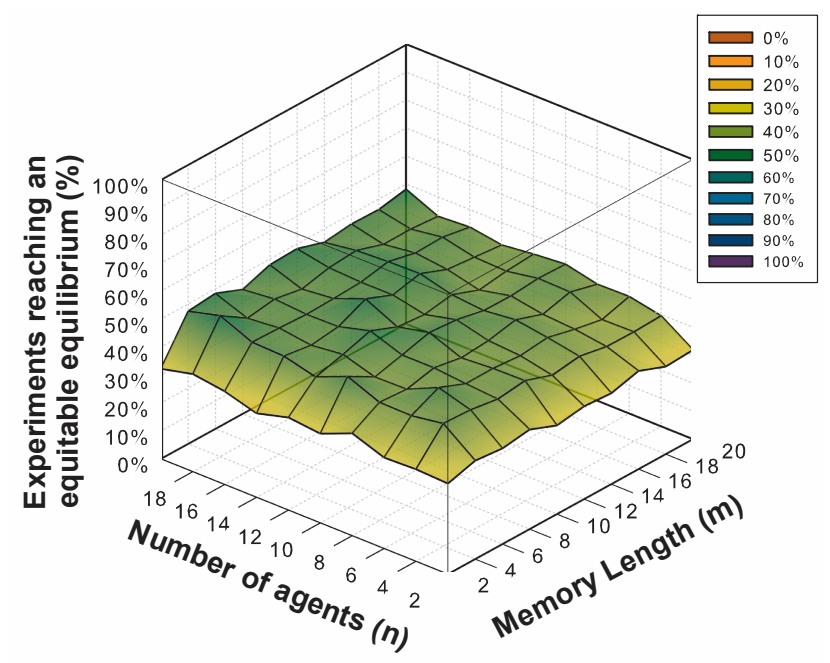

Figura 38. Porcentaje de experimentos que terminan en equilibrio paritario cuando se emplea la nueva regla de decisión. Se representa el valor promedio calculado a partir de 1.000 repeticiones de la simulación para cada par de valores (n, m). Parámetro de ruido $\varepsilon=0.2$. 
En el modelo original de AEY, los agentes no tenían limitaciones espaciales, por lo que cualquier agente podía ser emparejado con cualquier otro agente para negociar. Esta forma de interacción entre los agentes se aleja del escenario real de relaciones entre los seres humanos, en los que éstos tienden a interaccionar más frecuentemente con aquellos individuos que se encuentran en sus proximidades. Por esta razón, la siguiente extensión del modelo de AEY que llevamos a cabo consistió en situar a los agentes sobre un grid regular, como primer paso para añadir la dimensión espacial al modelo de AEY. Dicha extensión se desarrolla en la sección 3.4 y se encuentra publicada en (Poza et al., 2011).

\subsection{AGENTES SITUADOS EN UN GRID REGULAR}

En el modelo de AEY los agentes eran emparejados al azar. La consecuencia de este emparejamiento aleatorio es que cualquier par de agentes pueden interactuar entre sí, sin importar la distancia entre ellos. En la extensión del modelo que exponemos a continuación damos un primer paso para incluir en el modelo las limitaciones espaciales a las que están sometidos los agentes a la hora de interactuar entre sí.

Para ello, consideraremos que los agentes están situados sobre un grid regular, de tal manera que solamente podrán interactuar con sus ocho vecinos más próximos (vecindad de Moore). La Figura 39 muestra el grid regular empleado. Éste dispone de 10x10 celdas, dando cabida a 100 agentes. Puesto que la superficie es toroidal, todos los agentes tienen ocho vecinos, incluidos los agentes situados sobre las bandas del grid. En cada ronda, cada agente elegirá (al azar) a uno de sus ocho vecinos para llevar a cabo el juego de la demanda de Nash. 


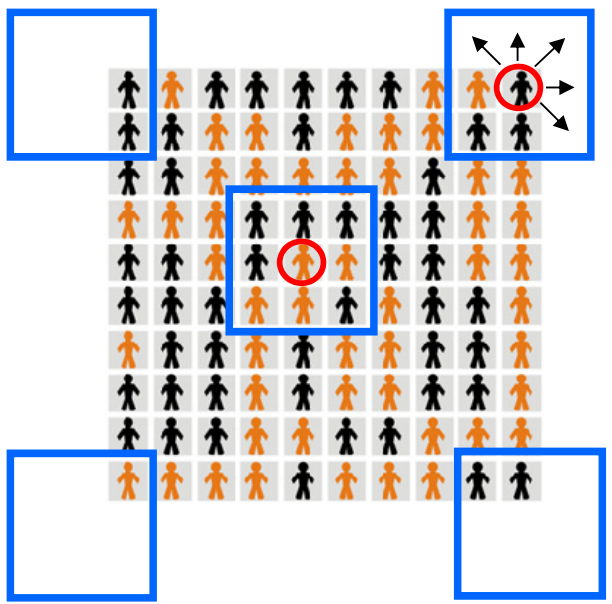

Figura 39. Grid toroidal empleado. Vecindad de Moore. Detalle de la vecindad de los agentes.

Dado que en este punto de la investigación se trata de averiguar si la introducción del grid permite alcanzar los mismos estados persistentes que se producían en el modelo de AEY, o incluso si se obtienen nuevos regímenes persistentes, en los sucesivos experimentos emplearemos la nueva regla de decisión introducida en la sección 3.3.3 (regla de decisión de la moda). La razón es que, como ya se había comprobado (ver sección 3.3.3.2), esta regla de decisión facilita la emergencia de regímenes distintos al equilibrio paritario.

En cuanto a la distribución de la tag de los agentes en el grid, inicialmente se consideraron las tres topologías que se muestran en la Figura 40: distribución en dos zonas (Figura 40a), distribución en cuatro zonas (Figura 40b) y distribución aleatoria (Figura 40c).

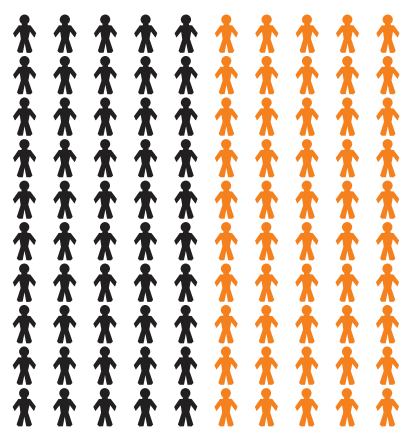

(a)

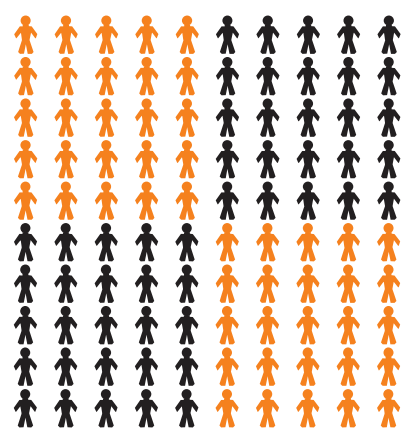

(b)

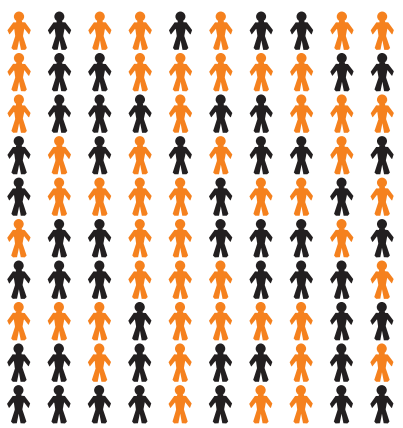

(c)

Figura 40. Tres distribuciones de los agentes en el grid según su tag. 
Sea cual sea la distribución de la tag en el grid, se ha considerado el mismo número de agentes que en el experimento con emparejamiento aleatorio: 100 agentes (50 de cada tag), para poder así comparar resultados y analizar la influencia del grid en el juego de la demanda de Nash.

Antes de analizar el caso más general (distribución de la tag de manera aleatoria), comenzaremos explorando los casos idealizados de distribución de la tag: en dos zonas y en cuatro zonas.

\subsubsection{DISTRIBUCIÓN DE LA $T A G$ EN DOS ZONAS}

Partimos de la distribución de la tag que se muestra en la Figura 40a. En las simulaciones que se llevaron a cabo, se observó que, además de poder obtenerse todos los estados persistentes que se deban en el caso de emparejamiento aleatorio, se podían obtener, además, otros resultados que anteriormente no se producían. La Figura 41 muestra los estados persistentes alcanzables cuando los encuentros se dan entre agentes del mismo tipo (juegos intratype). Téngase en cuenta que el color del agente representa su tag y el color de la celda sobre la que se encuentra cada agente indica la estrategia que representa la mejor respuesta ante la demanda esperada de su oponente (L-verde; M-amarillo; H-rojo).

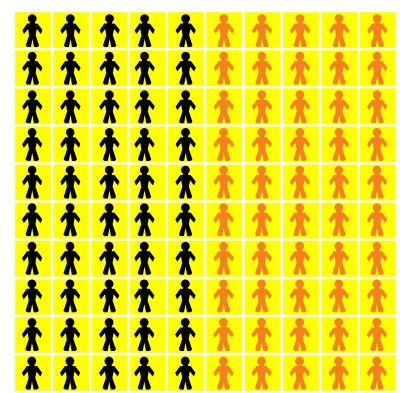

(a)

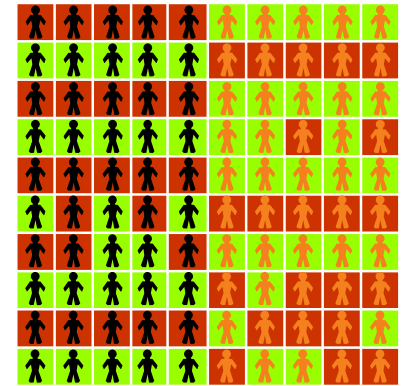

(b)

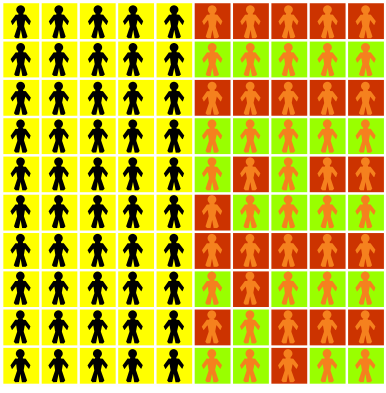

(c)

Figura 41. Posibles regímenes alcanzables en los juegos intratype ante una distribución de la tag en dos zonas.

En el caso de los juegos intratype, el sistema puede alcanzar un equilibrio paritario, independientemente de su tag (Figura 41a), un estado fluctuante independientemente de 
la tag (Figura 41b) o bien puede ocurrir que los agentes con una tag alcancen un equilibrio paritario y los agentes con la otra tag lleguen a un estado fluctuante (Figura 41c). Estos tres resultados son equivalentes a los obtenidos en el modelo de AEY con la regla de decisión original. Los simplex correspondientes a estos tres resultados son análogos a los que se mostraron en la Figura 31a, b y c, respectivamente ${ }^{35}$.

En lo que respecta a los encuentros intertype, pueden alcanzarse cuatro regímenes diferentes, los cuales se muestran en la Figura 42. Debe tenerse en cuenta que, debido a esta distribución particular de la tag, solamente los agentes que tienen como vecinos a agentes con una tag diferente a la suya pueden llevar a cabo el juego intertype. La celda sobre la que se encuentran los agentes que no pueden llevar a cabo encuentros intertype por esta razón se muestra en color gris.

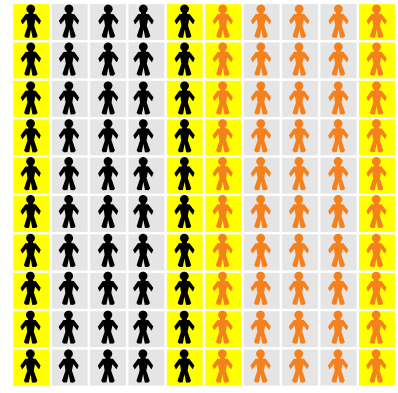

(a)

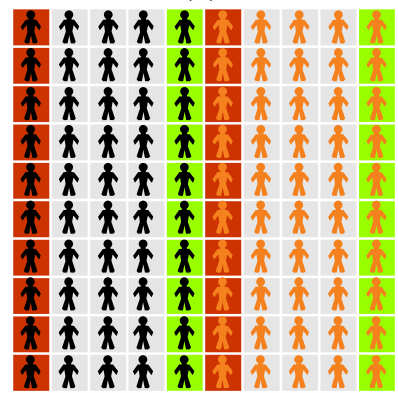

(c)

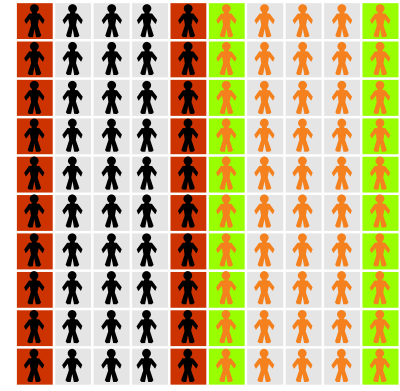

(b)

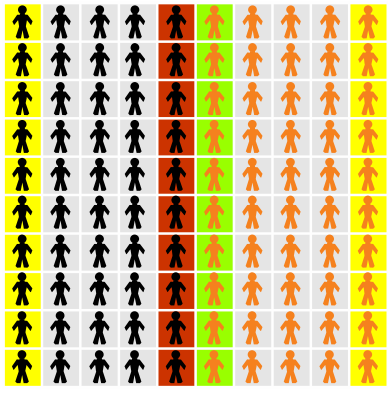

(d)

Figura 42. Posibles regímenes alcanzables en los juegos intertype cuando la tag se distribuye en dos zonas.

La Figura 42a y la Figura 42b muestran, respectivamente, un equilibrio paritario y un equilibrio segregado en las dos fronteras que permiten interacciones intertype. En ambos

\footnotetext{
${ }^{35}$ Nótese que, a pesar de que la posición de los agentes en el simplex sería equivalente, en este caso las fronteras de decisión no coincidirán con las de dichas figuras, ya que en estos experimentos se está empleando la regla de decisión de la moda en lugar de la regla de decisión original.
} 
casos, todos los agentes que tienen la misma tag alcanzan el mismo régimen. Estos dos resultados ya se daban en el modelo de AEY (de hecho, los simplex que representan el contenido de la memoria de los agentes serían similares a los de las Figura 32a y b, respectivamente $\left.{ }^{36}\right)$.

Sin embargo, la Figura 42c y la Figura 42d muestran resultados que no se obtenían cuando el emparejamiento era aleatorio. En ambos casos, agentes que tienen la misma tag alcanzan equilibrios diferentes en función de la frontera en la que se encuentren, pudiendo alcanzar, bien un equilibrio paritario, bien un equilibrio segregado. Esto contrasta con el caso original de emparejamiento aleatorio, en el que todos los agentes con la misma tag alcanzaban el mismo estado persistente.

Lo que ocurre es que cuando la tag se distribuye en dos zonas de la manera que muestra la Figura 40a y los encuentros son entre agentes de distinta tag (intertype), se forman dos grupos aislados de agentes. Cada uno de los grupos efectuará el juego de negociación de manera independiente, sin que las decisiones que se toman en uno de los grupos afecten a las decisiones que se toman en el otro grupo. Esto provoca que los regímenes que se alcanzan en cada uno de los grupos sean independientes entre sí.

A pesar de que la distribución de la tag en dos zonas (Figura 40a) es un caso idealizado, puede concluirse que, en función de cómo se encuentre distribuida la tag en el grid, pueden surgir grupos aislados de agentes (llamados componentes en la terminología de redes) que llevan a cabo la negociación de manera independiente, dando lugar a resultados que no se obtenían cuando el emparejamiento se realizaba de manera aleatoria. En la sección 3.4.3.2 se verá que no es necesario recurrir al caso idealizado de la Figura 40a para que se formen componentes, sino que éstas también pueden emerger en el caso general de distribución aleatoria de la tag (como en la Figura 40c).

\footnotetext{
${ }^{36}$ Nótese que, a pesar de que la posición de los agentes en el simplex sería equivalente, en este caso las fronteras de decisión no coincidirán con las de dichas figuras, ya que en estos experimentos se está empleando la regla de decisión de la moda en lugar de la regla de decisión original.
} 


\subsubsection{DISTRIBUCIÓN DE LA $T A G$ EN CUATRO ZONAS}

La distribución de la tag en dos zonas (Figura 40a), en el caso de los juegos intertype, daba lugar a dos grupos aislados de agentes que realizaban la negociación de manera independiente, de manera que existía la posibilidad de que estos grupos aislados de agentes, aun teniendo la misma tag, alcanzaran distintos regímenes debido a que su red de interacción impedía que hubiera flujo de información entre estos grupos.

Estos grupos aislados de agentes de la misma tag no pueden darse cuando la tag se encuentra distribuida en cuatro zonas (Figura 40b), ni cuando los encuentros son entre agentes del mismo tipo (intratype) ni cuando las negociaciones se dan entre agentes de distinta tag (intertype), ya que, en ambos casos, todos los agentes de la misma tag se encuentran interconectados.

Podría esperarse, entonces, que esta distribución de la tag no diera lugar a resultados distintos a los obtenidos con emparejamiento es aleatorio. De hecho, cuando se deja evolucionar el sistema durante un tiempo suficientemente largo (estado asintótico del sistema), los regímenes que se alcanzan son los mismos que cuando el emparejamiento es aleatorio, tal como se muestra en la Figura 43 (intratype) y en la Figura 44 (intertype). Nótese que, al igual que en el caso de la distribución de la tag en dos zonas, las celdas cuyos agentes no pueden realizar el juego intertype debido a que no tienen ningún vecino con el que puedan interactuar se muestran en color gris.

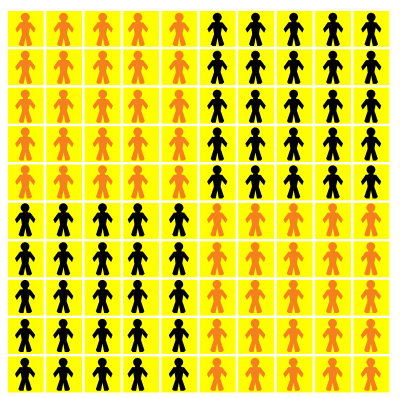

(a)

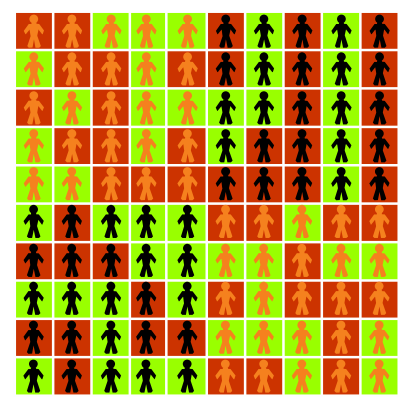

(b)

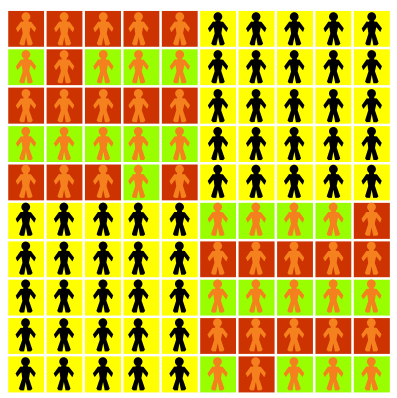

(c)

Figura 43. Posibles regímenes alcanzables en los juegos intratype ante una distribución de la tag en cuatro zonas. 


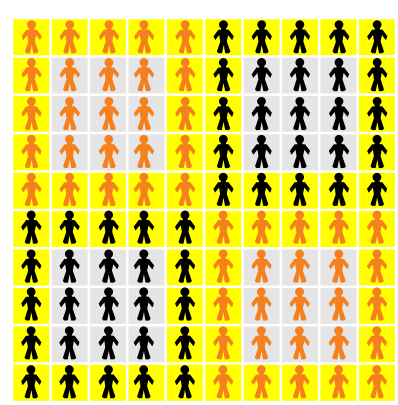

(a)

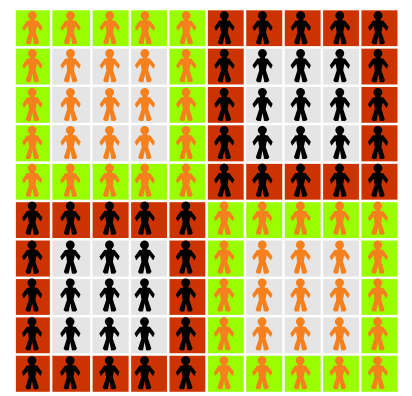

(b)

Figura 44. Posibles regímenes alcanzables en los juegos intertype cuando la tag se distribuye en cuatro zonas.

Como observamos, tanto en los juegos intratype (Figura 43) como en los juegos intertype (Figura 44), los regímenes que alcanza el sistema después de un tiempo de simulación suficientemente largo (estado asintótico del sistema) son los mismos que se alcanzaban cuando el emparejamiento era aleatorio.

Sin embargo, si observamos lo que ocurre en el sistema durante la fase transitoria ${ }^{37}$, en ocasiones se produce un fenómeno interesante como el que se muestra en la Figura 45.

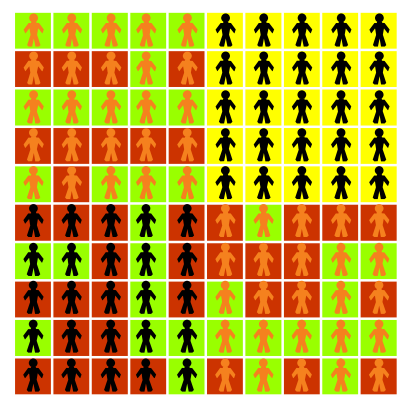

Figura 45. Estado del sistema en el que dos grupos de agentes de tag negra están coordinados, transitoriamente, en dos regímenes diferentes, a pesar de existir conexión entre ambos grupos.

En la Figura 45, todos los agentes con tag naranja se encuentran coordinados en el estado fluctuante. Sin embargo, un grupo de agentes de tag negro está coordinado en el equilibrio paritario y otro grupo se encuentra en el estado fluctuante. En este caso, la red de interacción de los agentes de tag negro sí permite el flujo de información entre

${ }^{37}$ En otras palabras, la Figura 45 muestra "una fotografía" del estado del sistema durante la ejecución de la simulación. 
ambos grupos (a diferencia del caso en el que la tag se encontraba distribuida en dos zonas y se formaban dos grupos aislados).

Lo que ocurre cuando la tag se distribuye de esta manera idealizada es que se forman dos grupos de agentes con tag negra cuyos agentes están densamente conectados entre sí, pero la conexión entre estos dos grupos es muy débil. Esta peculiaridad de la topología de interacción puede provocar que, transitoriamente, ambos grupos de agentes, a pesar de contar con la misma tag, se coordinen en regímenes distintos, como observamos en la Figura 45. Sin embargo, cuando se le permite al sistema evolucionar durante un tiempo suficientemente largo, la norma presente en uno de los grupos termina por invadir al otro grupo.

En conclusión, el caso idealizado de distribución de la tag en cuatro zonas (Figura 40b) da lugar a grupos de agentes con la misma tag que se encuentran densamente conectados dentro de su grupo, mientras que la conexión entre grupos es débil (esta topología recibe el nombre de estructura de comunidad en terminología de redes). Esto posibilita que distintos grupos de la misma tag puedan coordinarse, transitoriamente, en regímenes distintos, hasta que la norma en la que se encuentra coordinado uno de los grupos invade al otro grupo, de manera que todos los agentes con la misma tag acaban alcanzando finalmente el mismo régimen.

En la sección 3.4.3.2 observaremos que no es necesario recurrir al caso idealizado de la Figura 40b para generar estructuras de comunidad, sino que éstas también pueden emerger de manera espontánea cuando la distribución de la tag es aleatoria (como en la Figura 40c).

\subsubsection{DISTRIBUCIÓN DE LA TAG DE MANERA ALEATORIA}

En las secciones anteriores comprobamos que la distribución de la tag puede originar ciertas estructuras de red que dan lugar a resultados que no se daban cuando el emparejamiento de los agentes era aleatorio. Estas estructuras de red son las siguientes: 
varios componentes cuando la tag se distribuye en dos zonas (sección 3.4.1) y estructuras de comunidad cuando la tag está distribuida en cuatro zonas (sección 3.4.2).

En la presente sección se analizará el caso más general de distribución de la tag en el grid, es decir, una distribución aleatoria como la mostrada en la Figura 40c. Los experimentos anteriores demostraron que la red de interacción de los agentes es el elemento clave para que emerjan las estructuras de red que se pretenden analizar (componentes y comunidades). Por esta razón, en adelante, la red de interacción de los agentes se representará mediante grafos. Esto nos permitirá aplicar los conceptos y herramientas sobre redes que se han visto con anterioridad (sección 2.3).

Aunque el grid que se ha empleado en las simulaciones consta de 10x10 celdas, con el fin de ilustrar con claridad el concepto de red de interacción entre agentes, emplearemos en esta explicación el grid toroidal de $4 \times 4$ celdas que se muestra en la Figura 46. Éste ha sido generado mediante una distribución aleatoria de la tag.

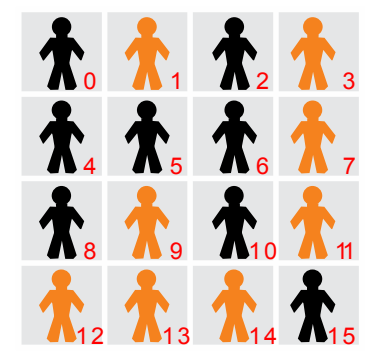

Figura 46. Grid toroidal de dimensiones $4 \times 4$ con distribución aleatoria de la tag.

La Figura 47 muestra la red de vecindad de los agentes de la Figura 46, teniendo en cuenta que cada uno de ellos puede interactuar con sus ocho vecinos más próximos (vecindad de Moore) y que el grid es toroidal. 


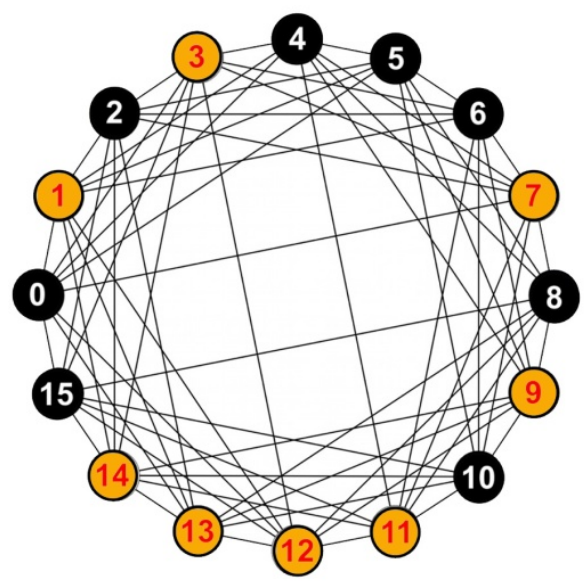

Figura 47. Red de interacción de los agentes de la Figura 46.

En la Figura 47, la presencia de un enlace entre dos nodos indica la relación de vecindad entre dos agentes. Sin embargo, la red de interacción, que es la base del juego, dependerá de si los encuentros son entre agentes del mismo tipo (juegos intratype) o entre agentes de distinto tipo (juegos intertype). Además, dentro del primer caso existen dos situaciones: los juegos intratype entre agentes de color naranja, y los juegos intratype entre agentes de color negro. Estas tres posibles redes de interacción se muestran en la Figura 48. 


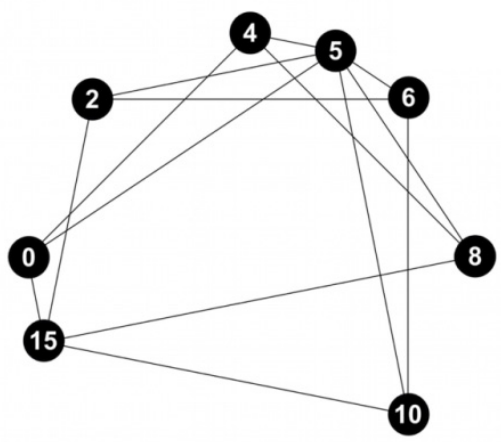

(a)

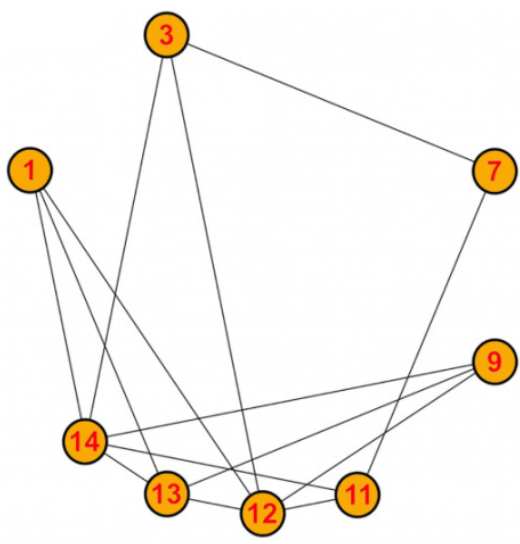

(b)

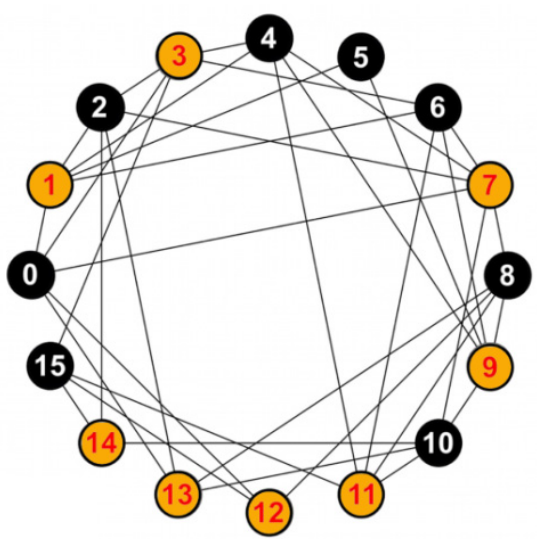

(c)

Figura 48. Redes de interacción entre los agentes de la Figura 46. (a) Juegos intratype entre agentes de color negro; (b) Juegos intratype entre agentes de color naranja; (c) Juegos intertype. 
Por lo tanto, se tienen tres tipos de juegos $y$, consecuentemente, tres redes de interacción distintas. Con el fin de definir el estado de cada uno de los tres juegos que tienen lugar de manera simultánea en el sistema, definiremos una tupla en la cual cada elemento indicará el régimen en el que se encuentra cada uno de los tipos de juego: \{Régimen intratype-negro, Régimen intratype-naranja, Régimen intertype\}. Como ya se había comprobado previamente, los posibles estados persistentes que pueden alcanzarse en cada tipo de juego son: equilibrio paritario (EQ), equilibrio segregado (IQ) y estado fluctuante (FR). Por lo tanto, al finalizar la simulación, esperamos que cada elemento de la tripla tome uno de estos valores ${ }^{38}$.

Al finalizar cada ronda de la simulación, se comprobará el régimen en el que se encuentra cada uno de los tres posibles juegos. Consideramos que una simulación ha concluido bien cuando se alcanza un determinado criterio de parada (estos criterios se mostrarán en las siguientes secciones) o bien cuando se alcanza el número máximo de rondas por simulación $\mathrm{T}_{\mathrm{f}}^{39}$.

Inicialmente (sección 3.4.3.1), se considerarán unas condiciones de parada (que llamaremos condiciones de parada C1) idénticas a las que se que se emplearon en el modelo con tags y emparejamiento aleatorio (ver Tabla 16). En dicha sección se comprobará que no todas las simulaciones finalizan con este criterio de parada, cosa que sí ocurría en el caso de emparejamiento aleatorio entre agentes. La razón está en que la distribución aleatoria de la tag en el grid puede dar lugar a la formación de componentes o estructuras de comunidad (como observamos de manera intuitiva en las secciones 3.4.1 y 3.4.2, respectivamente), situaciones que no son detectadas por las condiciones de parada C1. El motivo es que cada uno de los componentes o cada una de las comunidades (según el caso) pueden haberse coordinado en regímenes distintos, y las condiciones de parada $\mathrm{C} 1$ evalúan a todos los agentes con la misma tag.

En la sección 3.4.3.2 se introducirán unas nuevas condiciones de parada (que llamaremos condiciones de parada C2) que permitirán evaluar el régimen en el que se encuentra cada uno de los componentes que pudieran surgir en la red.

\footnotetext{
${ }^{38}$ Se espera que la tripla tome, al final de cada simulación, uno de las $3^{3}$ posibles combinaciones de estados: $\{\mathrm{EQ}, \mathrm{EQ}, \mathrm{EQ}\},\{\mathrm{EQ}, \mathrm{EQ}, \mathrm{IQ}\}$, etc.

${ }^{39} \mathrm{Se}$ ha considerado un número máximo de rondas por simulación $\mathrm{T}_{\mathrm{f}}=30.000$.
} 
Finalmente, en la sección 3.4.3.3, analizaremos los casos en los que surgen otros estados persistentes (como, por ejemplo, estructuras de comunidad) en los que, como veremos, el sistema alcanza el número máximo de rondas por simulación $\left(\mathrm{T}_{\mathrm{f}}\right)$ sin que se cumplan las condiciones de parada C1 ó C2. Cuando se alcance el número máximo de rondas por simulación sin que el sistema haya cumplido las condiciones de parada C1 ó C2 diremos que la simulación ha finalizado con criterio de parada C3.

\subsubsection{AUSENCIA DE COMPONENTES AISLADOS Y DE COMUNIDADES. CONDICIONES DE PARADA C1}

Se llevaron a cabo 10.000 repeticiones del experimento. La proporción de simulaciones que finalizaron con las condiciones de parada C1 (ver Tabla 16) fue 80,98\%. La frecuencia de simulaciones que finalizaron en un tiempo dado puede observarse en la Figura 49.

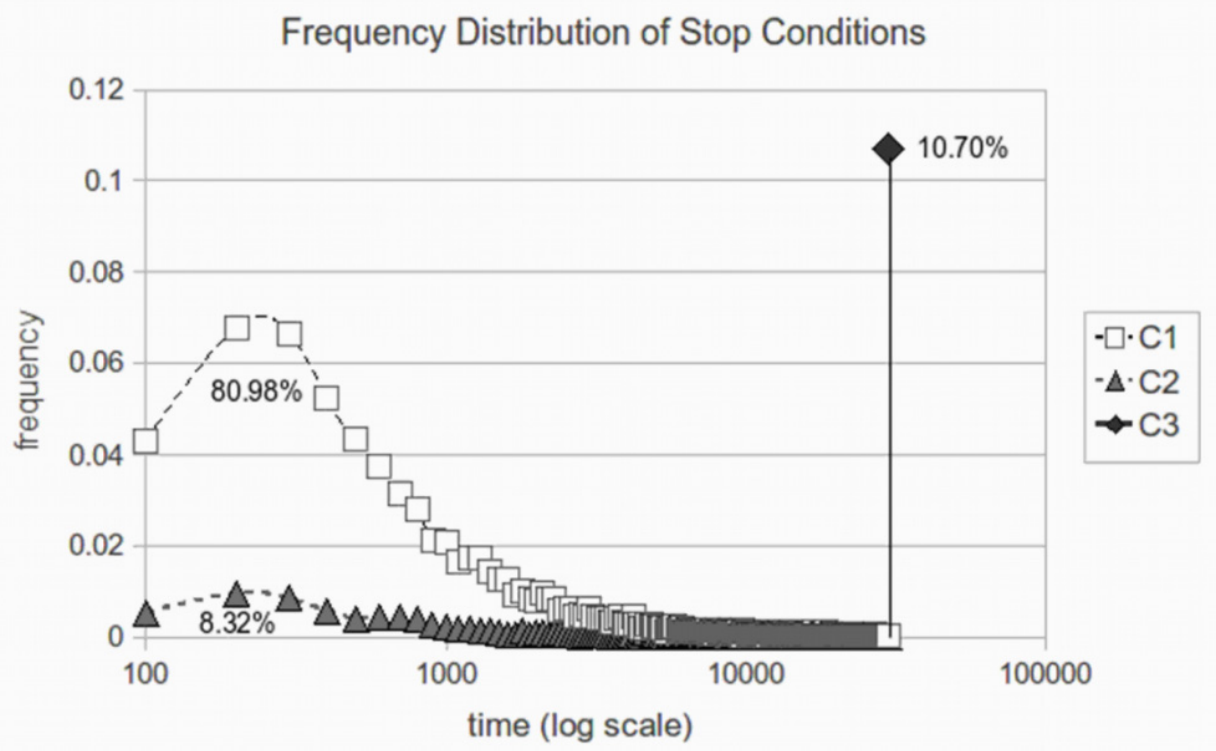

Figura 49. Frecuencia de simulaciones que alcanzaron las condiciones de parada C1, C2 ó C3 en un tiempo dado.

El hecho de que no todas las simulaciones finalicen con el criterio de parada C1 (que es el criterio de parada del juego original, cuando el emparejamiento era aleatorio) 
demuestra que, cuando la distribución de la tag es aleatoria, existen ocasiones en las que, a pesar de permitir que los agentes interactúen durante un tiempo suficientemente largo, el sistema no llega a alcanzar uno de los estados definidos en el modelo original de AEY. Esto se debe a la distribución aleatoria de la tag introduce ciertos efectos en la mesoscala (es decir, entre el nivel individual y el nivel de población) como por ejemplo, la aparición de componentes aislados (que veremos en la 3.4.3.2) o estructuras de comunidad (que analizaremos en la sección 3.4.3.3).

\subsubsection{COMPONENTES AISLADOS. CONDICIONES DE PARADA C2}

En ocasiones, la red de interacción de los agentes impuesta por la distribución aleatoria de la tag provoca que emerjan varios componentes aislados. Los agentes que forman parte de estos grupos aislados alcanzarán un régimen que no tiene por qué ser el mismo que los de otros componentes, ya que no existe comunicación entre grupos. Esto ya ocurría en el caso idealizado en el que la tag era distribuida en dos zonas (Figura 42c y d). La diferencia está en que en aquella ocasión la formación de componentes se hacía de manera intencional, mientras que en esta sección nos centraremos en el caso de los componentes aislados que surgen de manera espontánea cuando la tag es distribuida de manera aleatoria, analizado el impacto de la red de interacción resultante en los resultados de la simulación.

La Figura 50 muestra un grid en el que la tag ha sido distribuida aleatoriamente entre los agentes. 


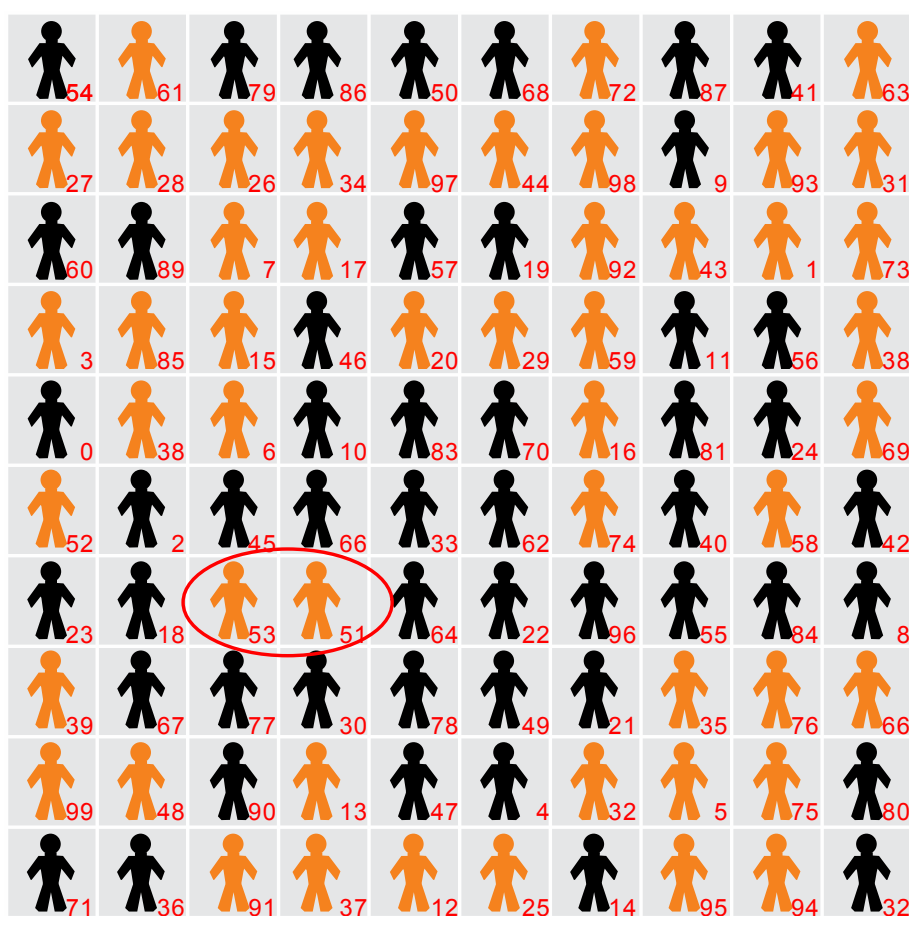

Figura 50. Ejemplo de distribución aleatoria de la tag que da lugar a dos componentes, en este casco en la red de interacción intraype de agentes naranjas.

La Figura 51 muestra la red de interacción intratype de agentes naranjas correspondiente al grid de la Figura 50. Obsérvese que se han originado dos componentes de agentes naranjas. 


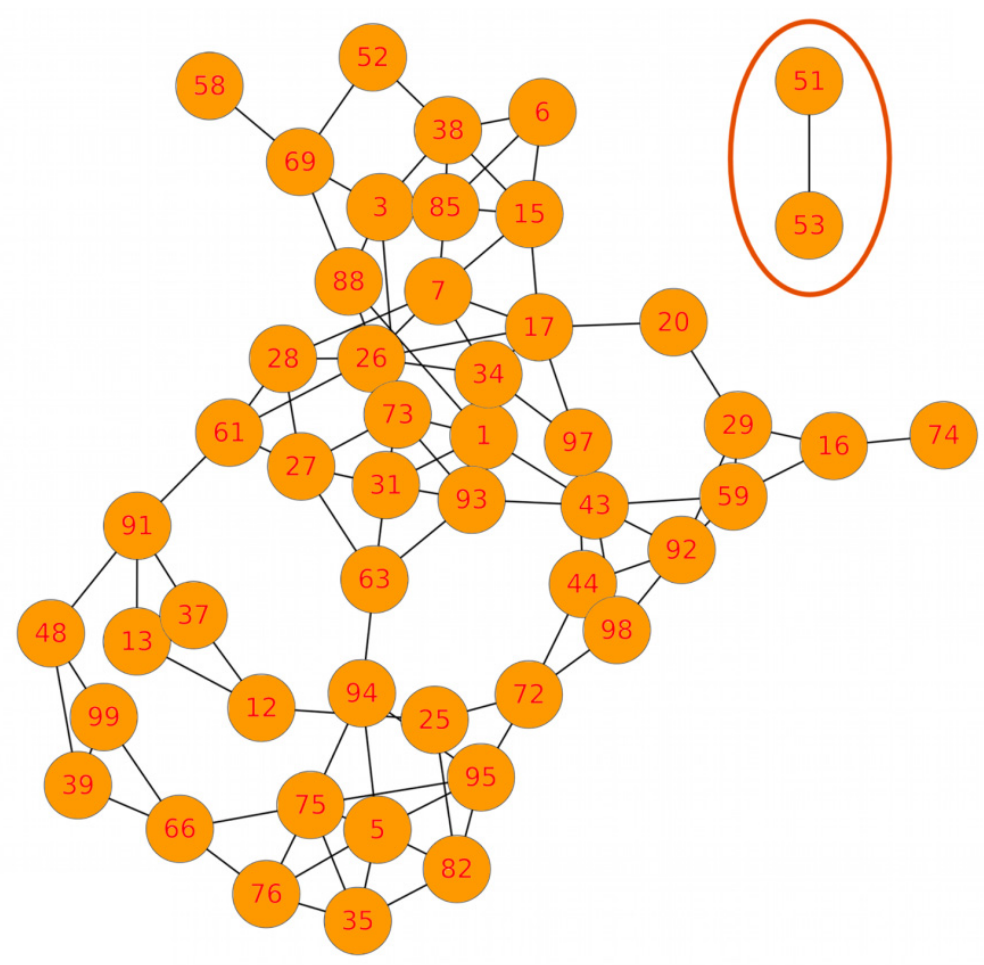

Figura 51. Red de interacción intratype de los agentes de color naranja del grid de la Figura 50.

Las condiciones de parada C1 (condiciones de parada originales, cuando el emparejamiento es aleatorio) no tenían en cuenta que pudieran surgir estas estructuras de red que provocan que, cuando todos los componentes no están coordinados en el mismo régimen, el sistema no cumpla con las condiciones de parada $\mathrm{C} 1$.

Por ello, el siguiente paso fue modificar las condiciones de parada para que se tuviera en cuenta el régimen en el que se encontraba cada uno de los componentes, de tal forma que la simulación se detuviera cuando todos los componentes del sistema hubieran alcanzado uno de los regímenes persistentes definidos con anterioridad (EQ, IQ, FR). Las nuevas condiciones de parada, a las que se dio el nombre de condiciones de parada $\mathrm{C} 2$, son la mismas que las que se empleaban en el modelo original (Tabla 16) sólo que en lugar de aplicarlas a toda la población, se aplicarán a nivel de componente.

Como puede apreciarse en la Figura 49, el porcentaje de simulaciones que finalizaron con el criterio de parada C2 fue 8,32\%. Por consiguiente, considerando simultáneamente condiciones de parada C1 y C2, la simulación finaliza antes de alcanzar el número 
máximo de simulaciones por ronda $\left(\mathrm{T}_{\mathrm{f}}=30.000\right.$ rondas $)$ en, aproximadamente, un $89 \%$ de los casos $(80,98 \%+8,32 \%)$.

El resto de las simulaciones $(10,7 \%$ ) alcanzó el número máximo de simulaciones por ronda $T_{\mathrm{f}}$ sin que el sistema hubiera alcanzado ningún estado que las condiciones de parada C1 y C2 pudieran detectar (es decir, finalizaron con condiciones de parada C3). En la siguiente sección analizaremos las posibles causas.

\subsubsection{OTROS ESTADOS PERSISTENTES. ESTRUCTURA DE COMUNIDAD. CONDICIONES DE PARADA C3}

Como puede apreciarse en la Figura 49, el 10,7\% de las simulaciones finalizaron con condiciones de parada C3, es decir, alcanzaron el número máximo de simulaciones por ronda $\left(\mathrm{T}_{\mathrm{f}}\right)$ sin que cumplieran las condiciones de parada $\mathrm{C} 1$ ó $\mathrm{C} 2$.

Una posible explicación para estos casos es que surjan estructuras de comunidad en las redes de interacción de los agentes como resultado de la distribución aleatoria de la tag en el grid. Particularizando la definición para el caso que nos ocupa, hablamos de estructura de comunidad cuando tenemos grupos de agentes de la misma tag que se encuentran conectados entre sí de una manera relativamente densa dentro de ciertos grupos, pero con una conectividad escasa entre grupos. Un caso idealizado de estructura de comunidad en juegos entre agentes de color naranja se muestra en la Figura 52.

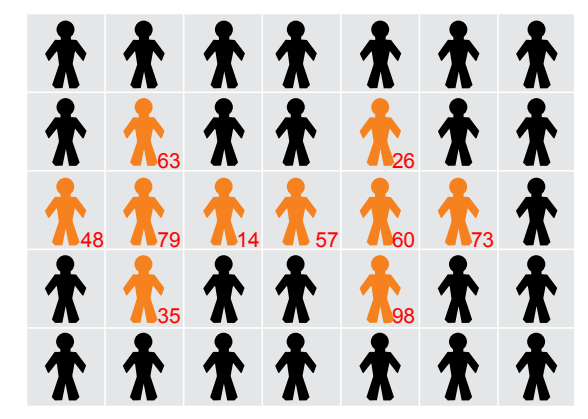

Figura 52. Caso idealizado de jugadores naranjas formando una estructura de comunidad en los juegos intratype. 
En la Figura 53 se muestra la red de interacción de los juegos intratype entre agentes de color naranja en la Figura 52. En ella se puede observar de manera intuitiva la existencia de dos comunidades, separadas a través de los nodos 14 y 57.

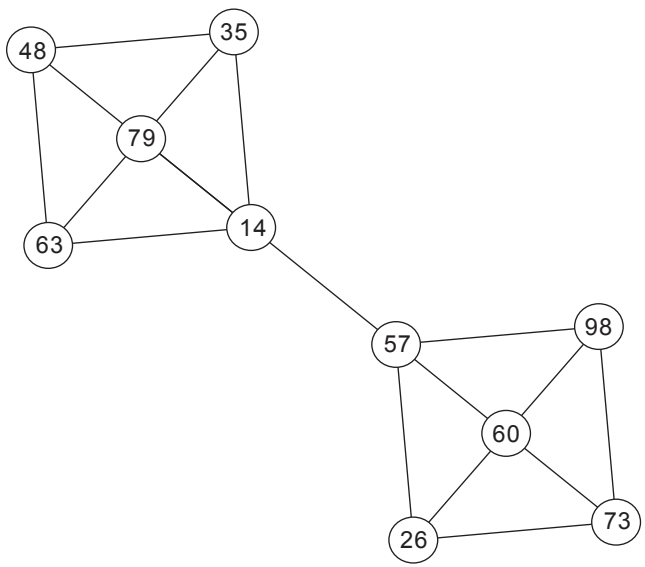

Figura 53. Red de interacción intratype entre los agentes de color naranja de la Figura 52.

Cuando los agentes de color naranja de la Figura 52 negocian entre sí, puede ocurrir que cada una de las comunidades se coordine en regímenes diferentes. Cuando esto ocurre, cada una de las comunidades puede permanecer atrapada en su régimen durante largos periodos de tiempo. La Figura 54 muestra un ejemplo de situación en la que cada una de las dos comunidades de la subred se encuentra coordinada en regímenes distintos.

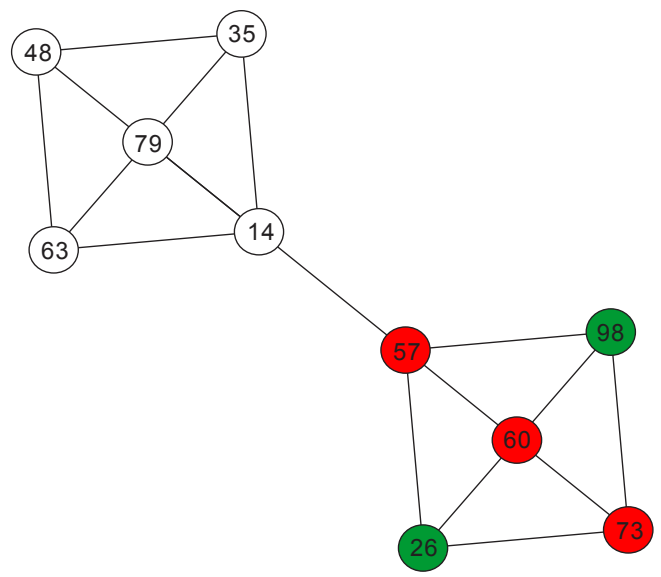

(a)

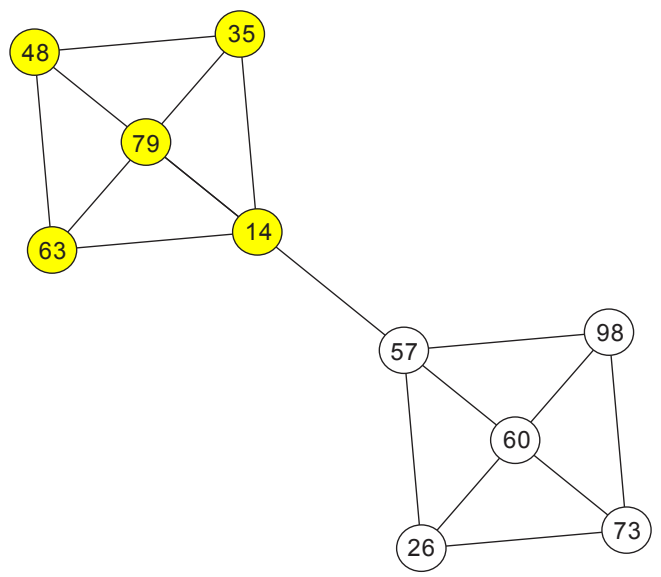

(b)

Figura 54. Instante en la que distintas comunidades de la red de la Figura 52 alcanzan regímenes diferentes. 
El hecho de que cada comunidad se encuentre coordinada en un régimen diferente dificulta que pueda alcanzarse un comportamiento homogéneo en todo el componente. La razón es que los agentes que hacen de frontera entre comunidades (en este caso los nodos 14 y 57) pueden presentar un comportamiento fluctuante dependiendo de la comunidad con la que jueguen en cada momento, haciendo de cuello de botella para la difusión de normas entre comunidades. En general, la frecuencia de interacción de esos agentes con los jugadores de la comunidad vecina va a ser más baja que con los jugadores de su propia comunidad. En términos de difusión de regímenes, este hecho es crucial, ya que para cambiar la estrategia necesitarían jugar muy a menudo con jugadores de la otra comunidad, pero esto está en contra de las posibilidades que impone la topología.

Con el objetivo de confirmar que el hecho de que una simulación finalice con criterio de parada C3 pueda deberse a la emergencia de estructuras de comunidad, las redes cuyas simulaciones finalizaron con este criterio de parada fueron tratadas por separado para su análisis.

En cada uno de estos casos (10,7\% tal como muestra el gráfico de la Figura 49) por un lado almacenamos el estado final de cada jugador en el juego intratype (EQ, IQ, FR o bien "régimen no establecido" si el agente no alcanza ninguno de los regímenes anteriores) y, por otro, exploramos estas redes en busca de estructuras de comunidad. Si la topología mesoscópica condiciona la difusión de estrategias, el estado de los jugadores debería ser homogéneo en cada comunidad excepto quizá en los nodos que hacen de frontera entre comunidades ${ }^{40}$.

Para la detección de comunidades se recurrió al algoritmo de Girvan-Newman. Nuestra hipótesis es que los enlaces que separan comunidades actúan de cuellos de botella que facilitan o ponen obstáculos al flujo de estrategias entre comunidades. Precisamente, el algoritmo de Girvan-Newman está basado en la idea de que los enlaces que conectan comunidades tienen alta betweenness en comparación con el resto y, por tanto, eliminándolos de manera iterativa, podremos separar diferentes componentes de la red que revelen la estructura de comunidad oculta en el grafo.

40 Definimos un nodo como frontera de una comunidad si éste tiene un enlace a otro nodo que pertenece a una comunidad distinta. 
El siguiente paso consistió en comparar las comunidades identificadas por el algoritmo de Girvan-Newman con el estado final de los agentes. Si nuestra hipótesis era acertada, los agentes que se encuentran en la frontera entre comunidades que se encuentran coordinadas en regímenes distintos deberían presentar una estrategia fluctuante, como consecuencia de la exposición a ambos regímenes, mientras que los agentes que no formen parte de la frontera deberían tener un comportamiento homogéneo de acuerdo con la comunidad a la que pertenecen.

Entre todas las simulaciones que finalizaron con el criterio de parada C3, se identificaron un total de 1007 componentes. La Figura 55 muestra el porcentaje de nodos explicados ${ }^{41}$ en dichas componentes. El número de nodos explicados de esta manera fue del 91,7\%.

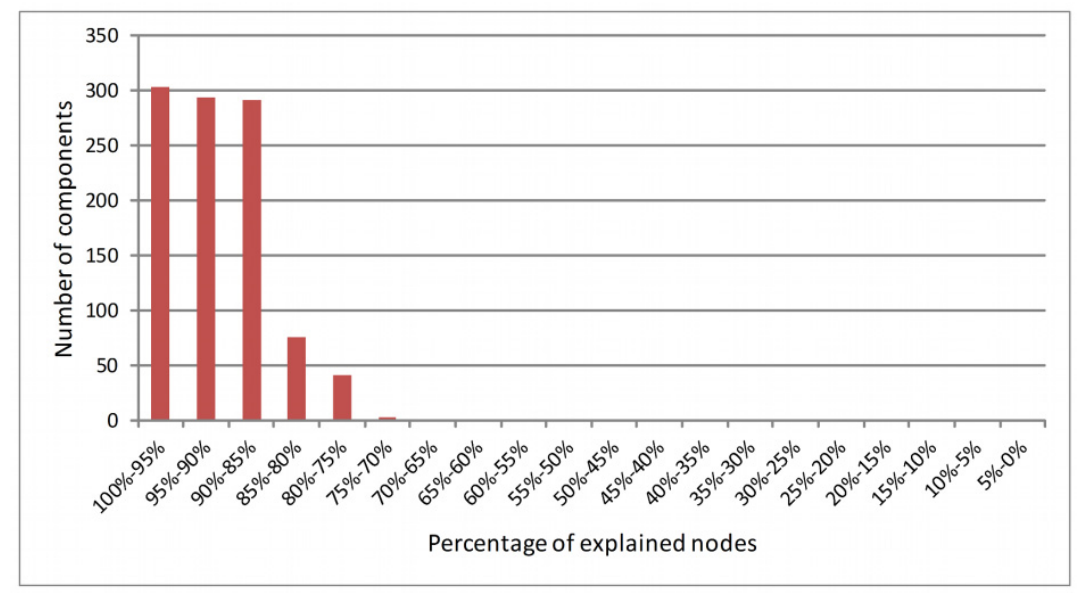

Figura 55. Número de componentes que tienen un porcentaje dado de nodos explicados. El número total de componentes identificadas en las simulaciones que finalizaron con condiciones de parada C3 fue de 1007.

Posteriormente, los 1007 componentes fueron particionados en 6.366 comunidades. La Figura 56 muestra el número de comunidades en función del número de nodos no explicados.

41 Nos referimos como "nodos explicados" a aquellos que, o bien tienen un comportamiento homogéneo de acuerdo con la comunidad a la que pertenecen, o bien tienen un comportamiento fluctuante porque se encuentran en la frontera entre dos comunidades coordinadas en regímenes diferentes. 


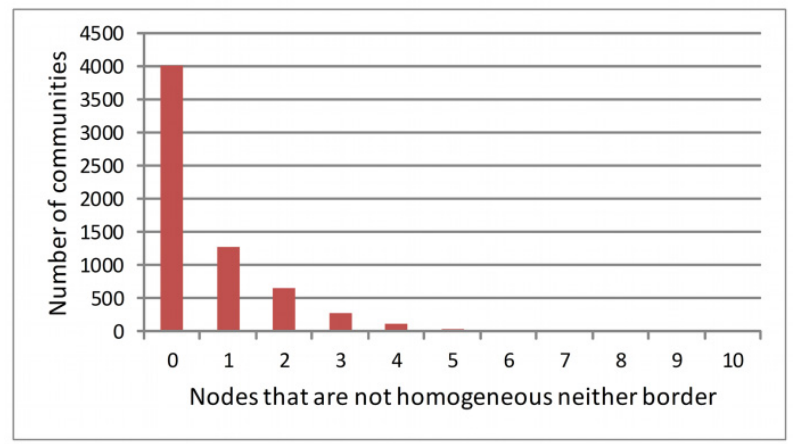

Figura 56. Número de comunidades identificadas por el algoritmo de Girvan-Newman en función del número de nodos que tiene un comportamiento discordante con el comportamiento esperado.

El porcentaje de nodos restante $(8,3 \%)$ tuvo una estrategia distinta a la esperada. Esto pudo deberse a varios motivos. En primer lugar, debe tenerse en cuenta que el modelo es estocástico y que, por tanto, la aleatoriedad puede introducir inercia en el estado de los jugadores. Un segundo factor podría ser que el número máximo de rondas por simulación que estamos considerando $\left(\mathrm{T}_{\mathrm{f}}=30.000\right)$ podría ser insuficiente para que algunas redes de topología compleja alcancen un régimen persistente. Finalmente, la partición de una red en comunidades depende del algoritmo empleado. Por tanto, es posible que el uso de otros algoritmos proporcione otras particiones que mejoren la explicación basada en el algoritmo de Girvan-Newman. 


\subsection{INFLUENCIA DE LA RED SOCIAL}

\subsubsection{INTRODUCCIÓN}

En el modelo de AEY el emparejamiento de los agentes era aleatorio. En un primer paso hacia un modelo más realista de interacción entre agentes, éstos fueron ubicados sobre un grid regular, de tal manera que la red de interacción de los agentes viniera impuesta por la topología del grid. Se comprobó que la distribución de la tag de los agentes en el grid daba lugar a redes de interacción con ciertas propiedades mesoscópicas que tenían un importante impacto en los regímenes que se alcanzaban en el sistema.

En esta sección presentamos una nueva extensión del modelo de AEY en la que se ha dado un paso más hacia una red de interacción más realista que la ofrecida por el grid regular: hacer que los agentes formen parte de una red social.

\subsubsection{PROPIEDADES DE LAS REDES SOCIALES Y MODELO EMPLEADO}

Las redes sociales presentan dos características fundamentales:

- Alto clustering: Existe una alta probabilidad de que dos nodos que sean vecinos tengan, a su vez, un vecino en común. En términos informales, en una red que presenta alto clustering existe una alta probabilidad de que los amigos de nuestros amigos sean, a su vez, amigos nuestros.

- Efecto de pequeño-mundo: La distancia geodésica entre dos nodos cualesquiera de la red es baja. Más informalmente: dos nodos cualesquiera de la red se encuentran separados por un número pequeño de saltos. 
El primer paso hacia el estudio del modelo de AEY sobre una red social será encontrar un modelo de red teórico que permita la generación de redes que cumplan con estas propiedades. Como ya se avanzó en la sección 2.3.5, las redes regulares presentan alto clustering, pero carecen del efecto de pequeño-mundo. Por otro lado, las redes aleatorias, si bien se caracterizan por su efecto de pequeño-mundo, carecen de la propiedad de alto clustering. El algoritmo ideado por Watts y Strogatz (1998), sin embargo, permite la creación de una familia de redes que pueden combinar ambas propiedades simultáneamente (ver Figura 19). Por consiguiente, éste será el algoritmo generador de redes que emplearemos en esta extensión del modelo de AEY.

El algoritmo de generación de redes de Watts y Strogatz se describió con anterioridad en la sección 2.3.5.3. Recuérdese que las redes generadas por este algoritmo presentan distintas propiedades de clustering y de distancia geodésica en función del parámetro $\beta$. Dicho parámetro, conocido como probabilidad de rewiring, puede tomar valores comprendidos entre 0 y 1 . Cuando $\beta=0$ se obtiene una red regular (con alto clustering y alta distancia geodésica), $y$, cuando $\beta=1$ se obtiene una red aleatoria (con bajo clustering y baja distancia geodésica). Sin embargo, para valores intermedios de $\beta$, las redes generadas pueden presentar las dos características de las redes sociales (alto clustering y baja distancia geodésica) de manera simultánea.

El objetivo de esta parte de la investigación el analizar cómo las propiedades de las redes generadas por el algoritmo de Watts y Strogatz (las cuales derivan del valor del parámetro $\beta$ con el que son generadas) influyen, tanto en la emergencia como en la difusión de la norma paritaria.

\subsubsection{DISEÑO DE EXPERIMENTOS}

En cuanto al diseño de experimentos, las simulaciones se han llevado a cabo con los mismos parámetros que los experimentos anteriores (casos de emparejamiento aleatorio y grid), con el fin de poder comparar resultados. A saber: 100 agentes $(N=100)$, todos ellos dotados de una memoria de tamaño $10(m=10)$ y un factor de ruido $\varepsilon=0.01$. 
La regla de decisión de los agentes consistió en elegir la mejor estrategia frente a la demanda más probable del oponente (ver descripción de esta regla de decisión en la sección 3.3.3). La razón de emplear esta regla de decisión en lugar de la original del modelo de AEY se debe a que, a pesar de que la introducción de la nueva regla de decisión no afectaba a los regímenes que podían alcanzarse en el sistema, como ya se había comprobado previamente, la nueva regla de decisión facilitaba la emergencia de regímenes distintos a la norma paritaria (compárese la Figura 37 con la Figura 38).

Las redes se generaron con un grado medio igual a $8^{42}$. Se emplearon los mismos criterios de parada que en el modelo en el que los agentes estaban ubicados en un grid regular.

A diferencia de las extensiones del modelo de AEY realizadas hasta el momento, en las que se consideraba que los agentes disponían de una tag que condicionaba su estrategia, en los siguientes experimentos se asumirá que tales tags no existen en el sistema. Esto se debe a que, como ya se ha visto tanto en el modelo con emparejamiento aleatorio como en el grid, la existencia de una tag que toma dos valores da lugar a tres tipos de juego: juegos intratype entre agentes cuya tag toma el valor 1; juegos intratype entre agentes cuya tag toma el valor 2; y juegos intertype entre agentes de distinta tag. Las redes generadas mediante el algoritmo de Watts y Strogatz presentan ciertas propiedades de clustering y distancia geodésica en función el parámetro $\beta$ con el que son generadas. La consideración de una tag nos obligaría a descomponer la red generada por el algoritmo de Watts y Strogatz en tres redes de interacción distintas, que, sin embargo, no tendrían por qué conservar las propiedades de clustering y distancia geodésica de la red original, cuando es precisamente la influencia de sus propiedades lo que se pretende analizar en esta parte de la investigación.

\subsubsection{INFLUENCIA DEL CLUSTERING EN LA EMERGENCIA DE LA NORMA PARITARIA}

Se generaron redes de Watts y Strogatz con los parámetros indicados anteriormente (100 agentes, tamaño de memoria $\mathrm{m}=10$, factor de ruido $\varepsilon=0.01$ ), recorriendo todo el rango

${ }^{42}$ Para $\beta=0$ y grado=8, las propiedades de la red resultante son próximas a las del grid regular analizado con anterioridad. 
de valores de $\beta$, con el fin de comprobar la influencia de este parámetro en los resultados. Por cada valor de $\beta$, el experimento se repitió 100 veces. Se midió el porcentaje de experimentos que alcanzaron el equilibrio paritario en función del parámetro $\beta$. El resultado puede observarse en la Figura 57.

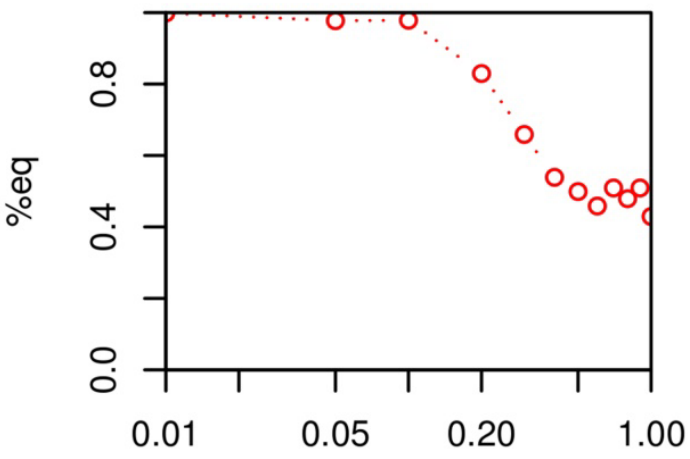

Figura 57. Porcentaje de experimentos que alcanzan la norma paritaria en función de $\beta$.

La Figura 57 nos permite comprobar que, conforme aumenta el valor de $\beta$, la probabilidad de emergencia de la norma paritaria va disminuyendo. Vemos, por tanto, que la emergencia del régimen paritario mantiene una fuerte dependencia con el valor de $\beta$, es decir, con la probabilidad de rewiring del algoritmo de Watts y Strogatz.

Sin embargo, lo que realmente nos interesa no es la dependencia de los resultados con $\beta$ (que es un parámetro del algoritmo generador de la red), sino que lo que buscamos analizar es la dependencia de los resultados con las propiedades de clustering y la distancia geodésica de la red (que, a su vez, dependen de $\beta$ ). Por tanto, para analizar cómo afecta el clustering y la distancia geodésica de la red a la emergencia de la norma paritaria, deberemos comparar el gráfico que muestra la probabilidad de emergencia de la norma paritaria (Figura 57) con las propiedades de la red en función de $\beta$, que se muestra en las Figura 58. 


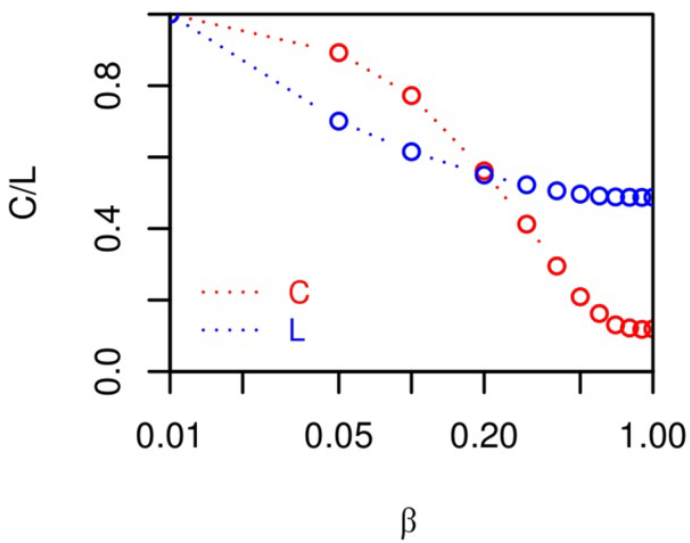

Figura 58. Coeficiente de clustering medio y distancia geodésica media de las redes generadas (ambos normalizados) en función de $\beta$. Nótese que, en comparación con el gráfico de la Figura 19, los datos con los que se ha construido esta gráfica se encuentran particularizados para nuestra red (100 nodos y 8 vecinos por nodo).

Como se observa en la Figura 58, conforme aumenta el valor de $\beta$, tanto el clustering como la distancia geodésica de la red disminuyen. Cuando $\beta=0$, la red resultante es regular, y presenta alto clustering y alta distancia geodésica. En este caso, la norma paritaria emerge en la mayoría de los casos $(98,84 \%)$. Si $\beta$ se incrementa hasta alcanzar su valor máximo $(\beta=1)$, la red resultante es una red aleatoria, caracterizada por bajos valores de clustering y distancia geodésica. En este caso, el porcentaje de experimentos que alcanza el régimen paritario constituye un mínimo dentro del rango de valores de $\beta$.

Sin embargo, en la Figura 58, observamos que existe un rango de valores de $\beta(0,05<\beta$ $<0,1$ ) en el que el clustering es alto (ha disminuido sensiblemente respecto a su valor máximo, que se produce para $\beta=1$ ) y, sin embargo, la distancia geodésica ha caído notablemente en comparación con su valor máximo. Para este rango de valores, el porcentaje de experimentos que alcanzan la norma paritaria (Figura 57) prácticamente no han variado respecto de su valor máximo (tan sólo ha disminuido sensiblemente). Por otro lado, si comparamos el clustering y la distancia geodésica de la red en este rango de valores de $\beta$ con sus valores mínimos (que se producen para $\beta=0$ ), observamos que el clustering en este rango es considerablemente superior, pero la distancia geodésica es tan sólo sensiblemente superior.

Por consiguiente, este experimento demuestra que la distancia geodésica de la red no explica la dinámica del sistema en el juego de negociación (al menos en redes pequeñas), lo cual tiene sentido, ya que la distancia entre agentes parece no jugar ningún papel en 
la manera en la que los agentes toman decisiones. Esto contrasta con el clustering de la red, que refleja la característica de vecindad, y que, por tanto, está relacionada con las interacciones de los agentes en la evolución de la negociación. Por tanto, queda demostrado que el clustering de la red influye fuertemente en la probabilidad de emergencia de la norma partitoria.

Para explicar de manera intuitiva por qué valores altos de clustering favorecen la emergencia de la norma paritaria, recurriremos al modelo idealizado de la Figura 59. En ella se muestran dos redes con tres nodos cada una. La única diferencia se encuentra en que en la red de la Figura 59a existe un enlace que une los nodos 1 y 2, mientras que este enlace no existe en la red de la Figura 59b. La red de la Figura 59a presenta alto clustering (de hecho, todos los nodos de esta red tienen vecinos en común), mientras que la red de la Figura 59b presenta clustering bajo (ningún nodo de esta red tiene vecinos en común). Se dice los tres nodos de la red de la Figura 59a se encuentran formando una triada, mientras que los de la Figura 59b forman un trío desconectado.

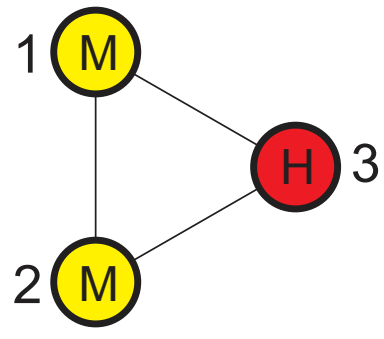

(a)

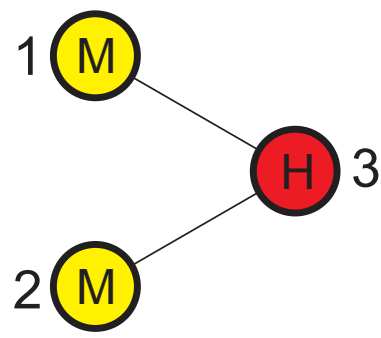

(b)

Figura 59. Efecto de la mutación dentro de una triada (a) y en un trío desconectado (b) cuando los agentes están coordinados inicialmente en la norma paritaria (M) y un nodo mutante (3) cambia su demanda de M (amarillo) a $\mathrm{H}$ (rojo).

Para comparar la robustez de las redes de la Figura 59, supondremos que, inicialmente, los tres nodos de cada una de las redes se encuentran coordinados en la norma paritaria (es decir, los tres demandan M). Supongamos también que, en cierto momento, el agente 3 sufre una mutación y cambia su demanda de $\mathrm{M}$ a $\mathrm{H}$. ¿Cuál será el impacto de esta mutación en las estrategias de los nodos 1 y 2 en función de la topología de la red (es decir, en función del clustering)?

Cuando los tres nodos se encuentran formando una triada (Figura 59a), la topología de la red de interacción de los agentes debilita el estado del mutante, quien tiene incentivos para volver a la estrategia M, ya que todos sus oponentes esperados demandan M. 
Además, los agentes 1 y 2 tienen incentivos para mantener su estrategia actual M, ya que ésta es exitosa en la mitad de sus posibles interacciones.

Sin embargo, cuando los nodos forman el trío desconectado de la Figura 59b, al no existir el enlace que une los nodos que demandan M (1 y 2), ahora, los agentes 1 y 2 sí tienen incentivos para cambiar su estrategia actual M por L, ya que la estrategia M, en la que estaban estabilizados, no es exitosa ante la estrategia $\mathrm{H}$ del nodo con el que mantienen la totalidad de los encuentros según esta topología de red.

Por tanto, un alto clustering hace que la norma paritaria sea más robusta ante mutaciones aleatorias.

Este ejemplo nos da una idea intuitiva de por qué la presencia de triadas facilita la emergencia de la norma paritaria. Obviamente, el análisis no es tan trivial cuando las redes poseen mayor tamaño y complejidad, pero a raíz de este ejemplo resulta intuitivo que el estado paritario es más robusto contra mutaciones cuando la red presenta alto clustering. Como consecuencia, cabe esperar que la norma paritaria se alcance más frecuentemente en aquellos casos en los que la red presenta alto clustering. Por tanto, en el caso concreto de las redes de Watts y Strogatz, esperamos una mayor probabilidad de emergencia de la norma paritaria cuando $\beta$ toma valores bajos, pues para estos valores de $\beta$, el clustering de las redes generadas es alto.

\subsubsection{INFLUENCIA DEL CLUSTERING EN LA DIFUSIÓN DE LA NORMA PARITARIA}

En la sección anterior concluimos que el hecho de que una red presente alto clustering favorece la emergencia de la norma paritaria. En esta sección mostraremos que, a pesar de ello, un alto clustering hace que la norma paritaria tarde más tiempo en difundirse a través de todos los nodos de la red.

Nuestra hipótesis de partida en cuanto al mecanismo de difusión de la norma paritaria a lo largo de la red es la siguiente: la población comienza en un estado aleatorio; en algún momento uno o más agentes escogen la estrategia paritaria y se coordinan en pequeños 
grupos que refuerzan sus estrategias. Finalmente, si uno de estos grupos presenta alguna propiedad particular (por hipótesis: tamaño y clustering), la norma paritaria acabará extendiéndose a toda la población.

Para comprobar si la hipótesis anterior es cierta, definimos una nueva unidad de análisis que denominamos núcleo paritario. Un núcleo paritario es un componente formado por agentes que demandarán $M$ en la siguiente interacción ${ }^{43}$. En un momento dado de la simulación puede haber ninguno, uno o varios núcleos paritarios en el sistema. Mediremos el tamaño y el clustering de los núcleos existentes en cada ronda de la simulación, con el propósito de correlar estas propiedades con la posterior evolución de los núcleos paritarios: bien crecer hasta invadir toda la población o bien decrecer hasta finalmente desaparecer. La Figura 60 muestra un núcleo paritario.

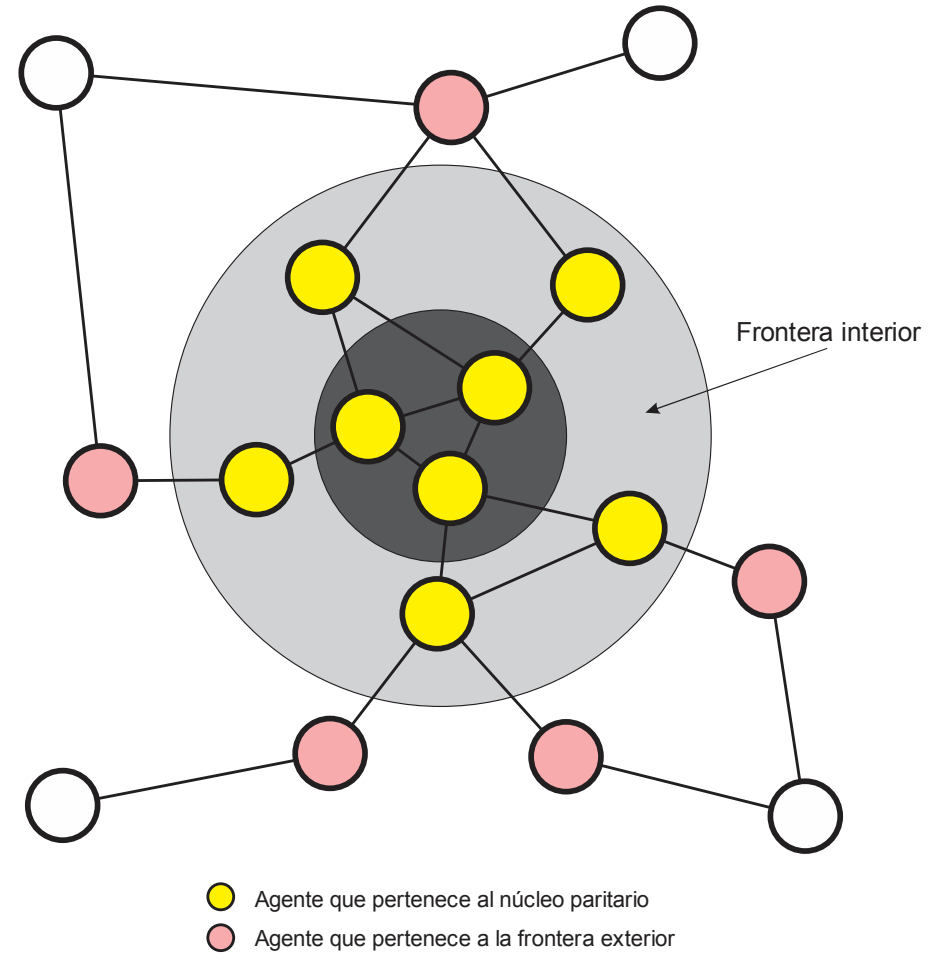

Figura 60. Núcleo paritario (nodos de color amarillo). La frontera interior del núcleo está sombreada en color gris claro. La frontera exterior del núcleo está constituida por los nodos de color rosa.

${ }^{43}$ Sin tener en cuenta el efecto del error en las decisiones. 
Basándonos en la definición de núcleo paritario, definimos dos conceptos adicionales:

- Frontera interior: Conjunto de agentes que pertenecen al núcleo paritario y que tienen al menos un vecino fuera del mismo. En otras palabras, los nodos que constituyen la frontera interior tienen al menos un vecino que no juega $\mathrm{M}$ en la siguiente interacción.

- Frontera exterior: Conjunto de agentes que no pertenecen al núcleo, pero que tienen al menos un vecino que sí pertenece al mismo.

Téngase en cuenta que cualquier cambio en el núcleo paritario involucra a las dos fronteras definidas anteriormente: si el núcleo incorpora nuevos nodos, lo hará cuando los miembros de la frontera exterior adopten la norma paritaria, mientras que el núcleo decrecerá cuando miembros de la frontera interior abandonen la norma paritaria.

Para comprobar el impacto del tamaño y el clustering de los núcleos paritarios en la difusión de la norma paritaria se llevó a cabo el siguiente procedimiento: en el inicio de cada ronda se identifican los núcleos paritarios que existen en el sistema en dicho instante (y sus correspondientes fronteras) y se computa su tamaño $\left(S_{i}\right)$ y su clustering $\left(C_{i}\right)$. Una vez completada la ronda se computa el nuevo tamaño $\left(S_{i}^{\prime}\right)$ y clustering $\left(C_{i}^{\prime}\right)$ de dicho núcleo. Con estos datos, $\left\{S_{i}, C_{i}, S_{i}^{\prime}, C_{i}^{\prime}\right\}$, se construye una matriz de cambio en la que se recogen el tamaño y el clustering de los núcleos paritarios existentes antes y después de cada ronda.

A partir de esta información, se generó un mapa de gradientes. Para ello, se descompuso el espacio tamaño-clustering en un grid cuadrado regular, de tal forma que, para cada celda, se computó la suma vectorial de todos los vectores con puntos iniciales en dicha celda, y se representó el vector resultante como una flecha de magnitud normalizada (puesto que sólo estamos interesados en la dirección del vector). Además, cada celda fue coloreada en función de la probabilidad de que un núcleo de tamaño $\mathrm{S}$ y clustering $\mathrm{C}$ aumente en tamaño. Esta probabilidad es computada como la frecuencia relativa de ocurrencia en los datos de la simulación.

La Figura 61 muestra el mapa de gradientes obtenido para varios valores del parámetro $\beta$. En él podemos observar que la difusión de la norma paritaria puede seguir dos comportamientos distintos en función de $\beta$ : 
- Comportamiento 1: Red regular $(\beta=0)$ y redes con baja probabilidad de rewiring $(\beta \leq 0.05)$ : el crecimiento es lento y homogéneo: la velocidad con la que los núcleos paritarios van invadiendo la red no presenta grandes variaciones a lo largo del tiempo de simulación. Ver Figura 61a, b y c. Puede comprobarse la predominancia del color verde (crecimiento lento) en dichas figuras.

- Comportamiento 2: Redes con mayor probabilidad de rewiring $(\beta \geq 0.2)$ : existe una primera etapa de desarrollo en la que el crecimiento de los núcleos es lento (celdas de color verde). Sin embargo, una vez el núcleo alcanza cierto tamaño crítico, la norma paritaria se extiende por toda la población de manera rápida (zona azul). Ver Figura 61e-l. 


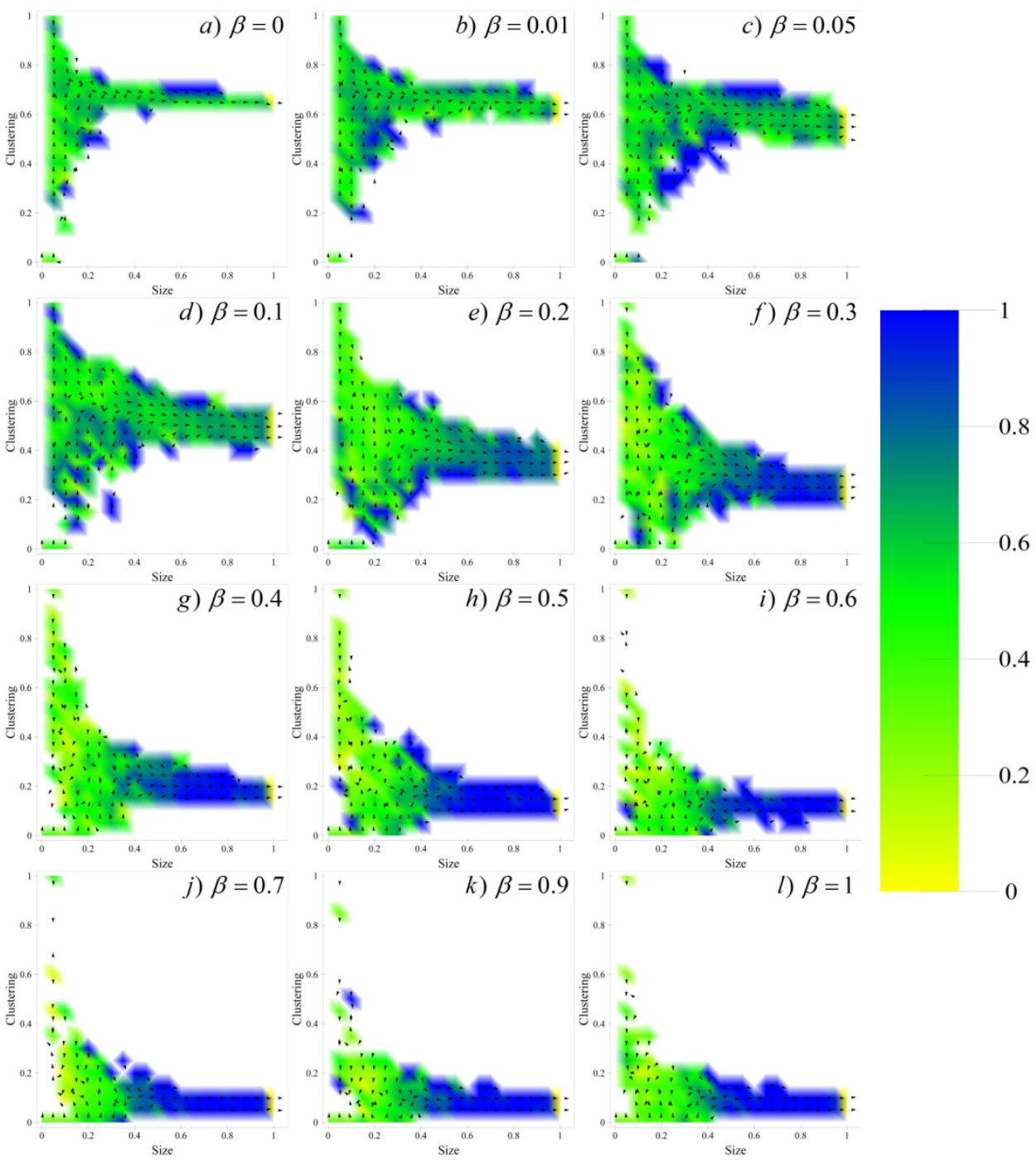

Figura 61. Mapa de gradiente de la dinámica de los núcleos paritarios para distintos valores de la probabilidad de rewiring $(\beta)$. Cada flecha representa la dirección del cambio del núcleo paritario en el espacio tamaño-clustering. El color de las celdas indica la probabilidad de crecimiento en tamaño del núcleo.

La razón de que el tiempo de difusión de la norma paritaria sea superior cuando el clustering es alto ( $\beta$ es bajo) se encuentra en que en estos casos los núcleos paritarios deben invadir subredes que también presentan alto clustering y que, posiblemente, se encuentren coordinadas en un régimen distinto al paritario, lo cual ralentiza el proceso de adopción. 
Sin embargo, cuando el clustering es más bajo (valores de $\beta$ más altos), el proceso de difusión es mucho más rápido ya que las subredes que deben invadir los núcleos paritarios presentan menos clustering.

La Figura 62 muestra el tiempo de difusión de la norma paritaria (entendido como el tiempo mínimo necesario para que toda la población converja hacia la norma paritaria, partiendo de condiciones iniciales aleatorias). Como se observa en dicha figura, conforme aumenta $\beta$, el tiempo de difusión disminuye, debido a la menor resistencia a la adopción de la norma paritaria debido al menor clustering de las redes invadidas. Debe tenerse en cuenta que los valores promedio que se muestran en la Figura 62 se han elaborado a partir de las simulaciones que alcanzaron la norma paritaria, y su número depende de $\beta$, como se vio en la Figura 57. Mientras que para valores pequeños de $\beta$ prácticamente todas las simulaciones alcanzaban la norma paritaria, este porcentaje disminuía hasta $80 \%$ para $\beta=0.2$ y hasta $50 \%$ para $\beta=1$.

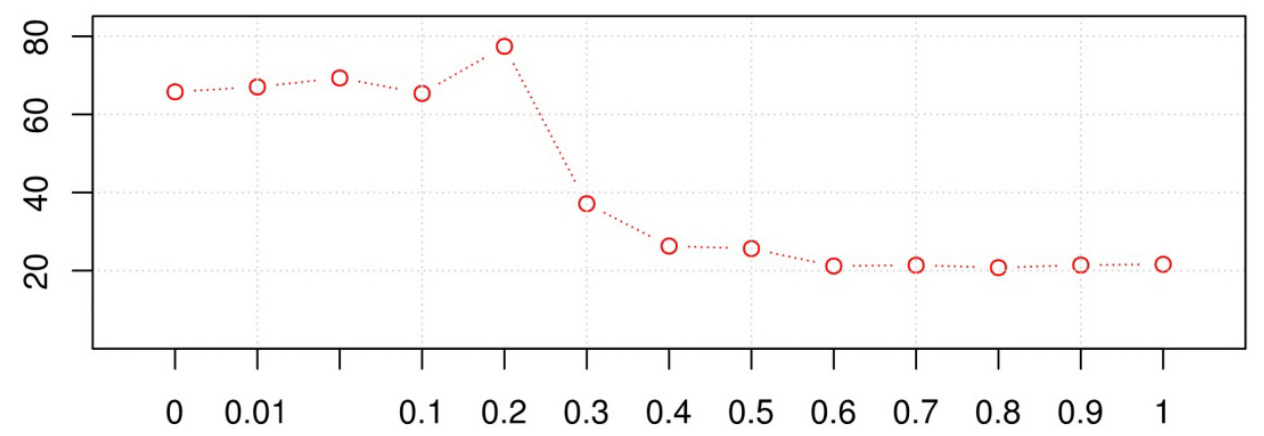

Figura 62. Tiempo medio de convergencia hacia la norma paritaria, en función de $\beta$

Según el gráfico de la Figura 62 parece haber una fase de cambio en el rango $0.1<$ $\beta<0.2$, lo cual coincide con un descenso significativo del clustering en la red (ver Figura 58). Esto explica la significativa dispersión en los tiempos de convergencia para $\beta=0.2$, que pueden observarse en la Figura 63. 


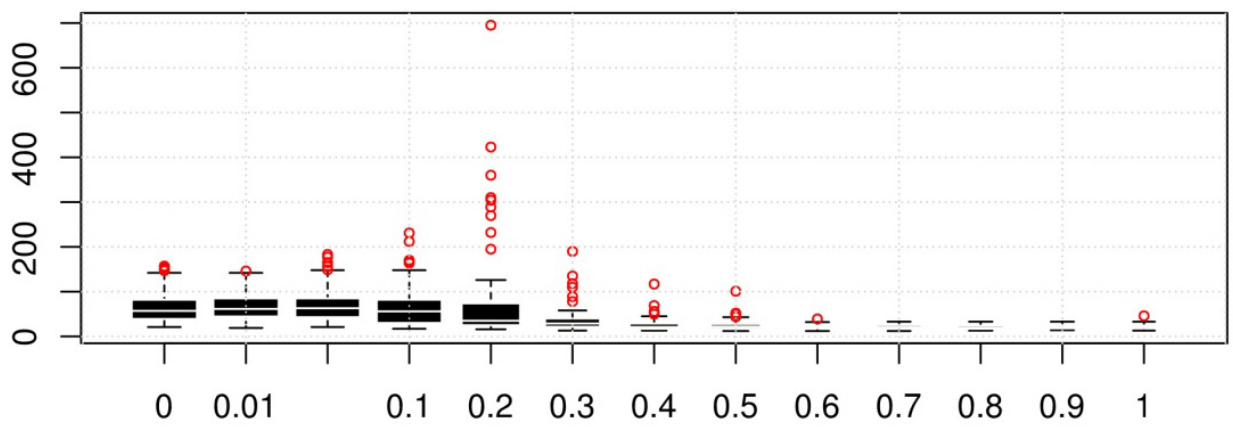

Figura 63. Diagrama de caja correspondiente al tiempo de convergencia hacia la norma paritaria, en función de $\beta$.

Como conclusión, las redes que presentan alto clustering facilitan la emergencia de la norma paritaria. Ahora bien, un alto clustering alarga el tiempo de difusión de la norma paritaria debido a que los núcleos que se encuentran coordinados en la norma paritaria han de invadir otras subredes con alto clustering que pueden encontrarse coordinadas en un régimen distinto al paritario, lo cual ralentiza el proceso de difusión.

Cuando el clustering de la red disminuye, la probabilidad de emergencia de la norma paritaria disminuye, pero, en los casos en los que la norma paritaria emerge, su tiempo de difusión es menor debido a que las subredes que han de invadir los núcleos tienen menor clustering. Esto hace que, una vez el núcleo paritario alcance un tamaño crítico, éste invada rápidamente al resto de la población. 



\section{CONCLUSIONES}

Consideramos oportuno diferenciar entre las conclusiones propiamente metodológicas y las debidas al problema concreto estudiado sobre la emergencia de normas económicas. Las primeras provienen de las lecciones extraídas de la utilización integradora de diferentes aproximaciones metodológicas: el Modelado Basado en Agentes (ABM), la Teoría de Juegos y la Teoría de Redes; tanto en el dominio específico del estudio de la emergencia de normas económicas como en el marco más general de los sistemas complejos. Las segundas corresponden a los resultados sobre el estudio del problema de negociación de Nash en sociedades de agentes artificiales. Finalmente exponemos algunas extensiones de esta investigación que consideramos oportunas como líneas de trabajo futuras.

\section{CONCLUSIONES METODOLÓGICAS}

El ABM permite estudiar comportamientos emergentes que no son predecibles a priori. El modelado tradicional, basado en modelos matemáticos y caracterizado por su elevado rigor formal, requiere asumir ciertas hipótesis y realizar simplificaciones para que las ecuaciones en las que se basan dichos modelos sean resolubles. Esto hace que, en ocasiones, sus resultados se alejen de lo observado en el mundo real. En el lado opuesto, el lenguaje natural, empleado tradicionalmente por las ciencias sociales, aporta una capacidad de descripción de la que carece el modelado matemático, si bien presenta el inconveniente de presentar poco rigor formal debido a su ambigüedad. El ABM se presenta como una alternativa a ambas aproximaciones, combinando las ventajas de ambas: rigor formal y realismo.

La replicabilidad de los experimentos es fundamental para el método científico en general, y también para el ABM en particular. Nuestra investigación se ha planteado con un sesgo importante hacia la validación y la replicabilidad de los resultados para asegurar el necesario rigor científico. Prueba de ello es que nuestro trabajo ha comenzado con la replicación del Modelo de Clases, modelo de partida de esta investigación. Se ha estudiado si los resultados obtenidos mediante nuestra réplica eran estadísticamente comparables con los obtenidos previamente en el modelo original. La replicación del modelo no sólo aporta rigor científico a este trabajo, sino que además 
permite mitigar la introducción de errores o artefactos en el modelo, posible limitación del ABM.

La Teoría de Juegos estudia las situaciones en las que el resultado no depende de la decisión tomada a nivel individual por cada agente, sino de las decisiones tomadas por todos los agentes en su conjunto. La introducción del ABM ha hecho posible que los agentes que intervienen en los dilemas sociales, modelados tradicionalmente como racionalmente perfectos según la Teoría de Juegos Clásica, puedan presentar distintas estrategias, no teniendo por qué ser éstas puramente racionales. Además, la introducción del ABM también ha facilitado que los agentes puedan aprender a partir de las interacciones con otros agentes en el pasado, adaptando sus estrategias en consecuencia.

La exhaustiva revisión de la bibliografía nos ha permitido constatar que existen numerosas líneas de investigación basadas en la combinación de Teoría de Juegos y ABM. Sin embargo, la mayoría de estos ABM asumen que los agentes son emparejados de manera aleatoria. Esto hace que, bien las interacciones, bien el aprendizaje o bien ambos se lleven a cabo sin tener en cuenta la posibilidad de la existencia de relaciones entre los agentes que condicionen sus interacciones.

En esta tesis doctoral se ha dado un paso más allá y se han considerado las limitaciones espaciales en cuanto a la posición de los agentes. Para ello hemos estudiado los resultados cuando los agentes se encuentran situados sobre un grid regular de tal forma que sólo pueden interactuar con sus vecinos. Hemos comprobado que las restricciones espaciales condicionan notablemente el resultado de los experimentos.

En una segunda etapa se ha considerado que los agentes forman parte de una red social que gobierna las posibles relaciones entre agentes. La estructura de la red social también condiciona los resultados observados en el juego de la demanda de Nash.

Si bien a partir de una revisión profunda de la literatura hemos observado que el ABM, la Teoría de Juegos y la Teoría de Redes se emplean habitualmente en sistemas complejos, también hemos constatado que en ningún trabajo hasta ahora se habían utilizado las tres aproximaciones metodológicas de manera simultánea en el campo de los problemas de negociación.

El uso integrado de ABM, Teoría de Juegos y Teoría de Redes presenta un gran potencial. La Teoría de Juegos facilita la formalización de situaciones estratégicas en la 
que el resultado es interdependiente de una manera estilizada. El uso de Teoría de Juegos (en el marco de juegos evolutivos y con aprendizaje) junto con ABM facilita el estudio de sistemas con poblaciones finitas, el análisis de la dinámica del sistema (no sólo resultados en equilibrio) y el análisis mediante simulación de regímenes persistentes más allá de los resultados asintóticos en el muy largo plazo, especialmente en modelos que presentan ergodicidad rota. Si al uso combinado de estas dos herramientas le añadimos la Teoría de Redes, podemos eliminar la hipótesis de interacción homogénea, aleatoria y completa, que, en muchos contextos son poco realistas, por otros modelos de interacción que presentan las regularidades encontradas en las redes sociales reales.

En nuestra investigación, el ABM, la Teoría de Juegos y la Teoría de Redes han sido aplicadas de manera conjunta a un modelo de negociación. La metodología integradora presentada en esta tesis doctoral permitirá el enriquecimiento de otros modelos en los que sea aplicada.

\section{CONCLUSIONES EN EL DOMINIO DE APLICACIÓN}

Nuestra investigación parte del Modelo de Clases de Axtell, Epstein y Young (AEY) (2000), un modelo de negociación bien conocido en el que una población de agentes es emparejada al azar para llevar al cabo el juego de la demanda de Nash. En este modelo se demuestra que el simple aprendizaje de los agentes a partir de sus interacciones con otros agentes en el pasado puede originar la emergencia de normas que regulan el intercambio entre los agentes. Las normas que pueden surgir en el sistema son dos: equilibrio paritario, en el que el excedente se reparte de manera equitativa entre los agentes y equilibrio segregado, en el que el reparto del excedente se hace de manera asimétrica. No obstante, en ocasiones el sistema puede alcanzar un estado fluctuante en el que no emerge ninguna norma en el sistema.

Nuestro primer paso consistió en la replicación del modelo y la comprobación de que los resultados obtenidos con nuestra réplica eran acordes a las conclusiones extraídas a partir del modelo original. Posteriormente, utilizamos nuestra réplica del modelo de AEY para llevar a cabo una serie de extensiones, cuyas conclusiones presentamos a continuación:

- En el modelo de AEY la segregación emerge espontáneamente, a pesar de que todos los agentes tienen la misma regla de comportamiento. El reconocimiento 
de la tag del oponente (que a priori no tendría por qué influir en la decisión de los agentes ya que se trata de una propiedad externa y sin implicación inicial) hace que los agentes "aprendan" cómo deben comportarse en función de la tag del agente con el que negocian en cada momento. Las simulaciones realizadas con nuestra réplica del modelo de AEY dieron lugar a resultados acordes con los obtenidos por los autores del modelo original.

- Las modificaciones del modelo de AEY sin tags que realizamos a partir de nuestra réplica del modelo mostraron interesantes resultados:

- El hecho de sustituir la regla de decisión original (que maximiza la utilidad utilizando la media) por otra que proporciona la mejor respuesta frente a la estrategia esperada más frecuente del oponente (que utiliza la moda) provoca importantes cambios en los resultados de la simulación. Si bien el conjunto de estados asintóticos del sistema no varía (no se produce variación en los regímenes alcanzables por el sistema), la dinámica del sistema sí se ve afectada: cuando los agentes emplean la regla de decisión de la moda, la proporción de experimentos que alcanzan el equilibrio paritario se reduce considerablemente.

- Las modificaciones en la matriz de pagos original da lugar a una notable modificación en el tiempo difusión: a mayor valor asignado a low (porción pequeña de la "tarta"), mayor es el tiempo que el sistema tarda en alcanzar el equilibrio paritario.

- El hecho de inicializar los agentes con memoria progresiva en lugar de con la memoria de tamaño fijo empleada en el modelo original provoca un escenario interesante: inicialmente, los agentes tienden a ser bien agresivos, bien pasivos, sin embargo, tras una serie de interacciones, aprenden a comprometerse y acaban coordinándose en la norma paritaria. La falta de coordinación de los agentes durante las primeras etapas de la simulación hacen que el tiempo de difusión de la norma aumente en comparación con el modelo original. 
- En cuanto al modelo con tags, la incorporación de la regla de decisión de la moda da lugar a una mayor frecuencia en la emergencia de equilibrios segregados que cuando se emplea la regla de decisión original basada en la media.

- Hemos demostrado que la topología explica la emergencia de nuevos regímenes diferentes a los observados en el modelo original, aparte de los que podían alcanzarse en el modelo con emparejamiento aleatorio:

- En concreto, la distribución de la tag de los agentes dentro del grid regular puede dar lugar a subredes de interacción que presentan ciertas propiedades mesoscópicas que explican la difusión de las estrategias de los agentes:

- Pueden surgir clústers de agentes que llevan a cabo el juego de la demanda de Nash de manera aislada, por lo que cada uno de ellos puede coordinarse en un régimen diferente sin tener en cuenta el régimen en el que se encuentran coordinados el resto de los clústers.

- La presencia de estructura de comunidad en la red de interacción provoca que cada una de las comunidades puedan permanecer coordinadas en distintos regímenes durante largos periodos de tiempo.

- Al situar los agentes en una red social, demostramos que las redes localmente estructuradas (en el sentido de que tienen mayor clustering) facilitan la emergencia de la norma paritaria, mientras que las redes menos estructuradas localmente facilitan la emergencia de estados fluctuantes. Por otro lado, observamos que el clustering de la red puede ralentizar la difusión de la norma paritaria, dificultando el proceso de adopción por parte de los agentes. En el caso de redes cuasi-regulares, la norma paritaria siempre emerge en el sistema, a pesar de que el tiempo que ésta tarda en difundirse es mayor. Sin embargo, en redes con un mayor grado de aleatoriedad, la probabilidad de alcanzar la norma paritaria decrece, si bien cuando ésta emerge se difunde rápidamente a todo el sistema. 


\section{EXTENSIONES}

En esta investigación se ha trabajado con redes que presentan dos de las características que pueden encontrarse en las redes sociales reales: alto clustering y baja distancia geodésica. No obstante, las redes sociales reales tienen otras propiedades adicionales, como son la asortatividad y la presencia de hubs.

En futuros trabajos revisaremos el juego de la demanda de Nash en redes que presentan estas propiedades, tanto de manera aislada como de manera conjunta, utilizando para ello el algoritmo de Holme (2002), que permite la creación de redes con una distribución de grado que sigue una ley de potencias en las que además el clustering se puede controlar. Además, se llevarán a cabo experimentos en los que se sustituirán las redes artificiales con las que hemos trabajado hasta el momento por redes reales (Lozano, Arenas \& Sánchez, 2008, 2008; Roca et al., 2010).

Consideramos necesario plantear otros algoritmos de aprendizaje partiendo de los resultados que proceden del avance de la Neuroeconomía, además de enriquecer la consideración de heterogeneidad en el tipo de agentes y la aplicación de esta metodología a otros tipos de interacción cuya esencia pueda ser capturada mediante juegos.

Finalmente, es nuestro deseo que este trabajo constituya el punto de partida a partir del cual sea posible explorar la generalidad y los límites de aplicación de nuestros resultados a la emergencia de normas socio-económicas en contextos más complejos. 


\section{REFERENCIAS}

AGUILERA ONTIVEROS, A. \& A. LÓPEZ PAREDES (2001) Modelado multiagente de sistemas socioeconómicos: una introducción al uso de la inteligencia artificial en la investigación social. s.l., San Luis Potosí: El Colegio de San Luis.

AGUILERA, A. (2002) Ciudades como tableros de ajedrez: introducción al modelado de dinámicas urbanas con autómatas celulares. s.l., San Luis Potosí: El Colegio de San Luis.

AGUILERA, A. \& A. LÓPEZ-PAREDES (2004) Sociedades Artificiales: Aplicación de la simulación en la teoría social. s.l., San Luis Potosí: Fac.de Ciencias-UASLP,

ALEXANDER, J. \& B. SKYRMS (1999) "Bargaining with Neighbors: Is Justice Contagious?". In The Journal of Philosophy. Vol. 96, N 11, November. Págs. 588.

ANBARCI, N. \& N. FELTOVICH (2010) "How sensitive are bargaining outcomes to changes in disagreement payoffs ?". In Journal of Economic Literature.

ARTHUR, W. B.; S. N. DURLAUF \& D. A. LANE (1997) The Economy as an Evolving Complex System II. ARTHUR, W. B.; S. N. DURLAUF \& D. A. LANE (EDS.), s.l., Addison-Wesley, Acfanced Book Program.

AXELROD, R. (1997) "Advancing the art of simulation in the social sciences". In Complexity. CONTE, R.; R. HEGSELMANN \& P. TERNA (EDS.). Vol. 3, ํo 2, Págs. 16-22. Springer.

AXELROD, R. (1997) The Complexity of Cooperation: Agent-Based Models of Competition and Collaboration. ANDERSON, P. W. et al. (EDS.), s.l., Princeton University Press.

AXTELL, R. (2000) "Why agents? On the varied motivations for agent computing in the social sciences". In Center for Social and Economic Dynamics Working. s.d. $\mathrm{N}^{\circ}$ 17, Págs. 17. Citeseer.

AXTELL, R. et al. (1996) "Aligning simulation models: A case study and results". In Computational \& Mathematical Organization Theory. Vol. 1, № 2, Págs. 123-141. Springer Netherlands. 
AXTELL, R.; J. M. EPSTEIN \& H. P. YOUNG (2000) "The Emergence of Classes in a Multi-Agent Bargaining Model". In Young. s.d. $\mathrm{N}^{\circ}$ 9,

BARABÁSI, A. L. \& R. ALBERT (1999) "Emergence of scaling in random networks". In Science. Vol. 286, $\mathrm{N}^{\circ}$ 5439, Págs. 509. American Association for the Advancement of Science.

BARABÁSI, A.-L. (2002) Linked: The New Science of Networks. s.l., Perseus Publishing.

BAUER, A. L.; C. A. A. BEAUCHEMIN \& A. S. PERELSON (2009) "Agent-based modeling of host-pathogen systems: The successes and challenges". In Information Sciences. Vol. 179, N 10, April. Págs. 1379-1389.

BIANCONI, G. \& A.-L. BARABÁSI (2001) "Competition and multiscaling in evolving networks". In Europhysics Letters. Vol. 54, º 436,

BONABEAU, E. (2002) "Agent-based modeling: Methods and techniques for simulating human systems". In Proceedings of the National Academy of Sciences of the United States of America. Vol. 99, N Suppl 3, Págs. 7280-7287. National Acad Sciences.

BOUSQUET, F. \& C. LE PAGE (2004) "Multi-agent simulations and ecosystem management: a review". In Ecological Modelling. Vol. 176, ํ3-4, Págs. 313-332. Elsevier.

COLEMAN, J. S. (1990) Foundations of Social Theory. s.l., Harvard University Press.

CONTE, R. (1997) Simulating Social Phenomena. CONTE, R.; R. HEGSELMANN \& P. TERNA (EDS.), s.l., Springer.

CONTRERAS, F. et al. (2002) "Teoría de juegos". In Investigación Operativa. Conferencia de 2002.

DANON, L. et al. (2005) "Comparing community structure identification". In Journal of Statistical Mechanics: Theory and Experiment. Vol. 2005, № 09, September. Págs. P09008-P09008. 
DESSALLES, J. L. (2007) "Emergence in multi-agent systems: conceptual and methodological issues". In Simulation. AMBLARD, F. \& D. PHAN (EDS.). Págs. 327-355. The Bardwell-Press.

EASLEY, D. \& J. M. KLEINBERG (2010) "Networks, Crowds, and Markets: Reasoning About a Highly Connected World". In Networks. Vol. 1,, Págs. 23-46. Cambridge University Press.

EDMONDS, B. (2001) "The Use of Models - making MABS actually work". In Multi Agent Based Simulation, Lecture Notes in Artificial Intelligence. MOSS, S. \& P. DAVIDSSON (EDS.). . p.15-32, Págs. 15-32. Springer.

EDMONDS, B. \& D. HALES (2009) "Computational simulation as theoretical experiment". In Journal of Mathematical Sociology. Vol. 29, N³, Págs. 209-232. Taylor \& Francis Inc.

EDMONDS, B. \& D. HALES (2005) "Computational simulation as theoretical experiment". In Journal of Mathematical Sociology. Vol. 29, N 3, Págs. 209-232. Taylor \& Francis.

EDMONDS, B. \& D. HALES (2003) "Replication, replication and replication: Some hard lessons from model alignment". In Journal of Artificial Societies and Social. Vol. 6, N 4, Págs. U227-U253.

EPSTEIN, J. M. (2001) "Learning to be thoughtless: Social norms and individual computation". In Computational Economics. Vol. 18, Nº 1, Págs. 9-24. Springer.

EPSTEIN, J. M. (1999) "Agent-based computational models and generative social science". In Complexity. Vol. 4, N 5, Págs. 41-60.

EPSTEIN, J. M. \& R. AXTELL (1996) Growing Artificial Societies from the Bottom Up. s.l., MIT Press \& Brookings Institution Press.

ERDÖS, P. \& A. RÉNYI (1959) "On random graphs". In Publicationes Mathematicae. Vol. 6, N 290-297, Págs. 290-297. Springer.

FUDENBERG, D. \& D. K. LEVINE (1998) "The Theory of Learning in Games". In Levines Working Paper Archive. Págs. 292. The MIT Press. 
FUENTES-FERNÁNDEZ, R. et al. (2011) "Metamodelling for Agent-Based Modelling: An Application for Posted Pricing Institutions". In Studies in Informatics and Control. Vol. 20, $\mathrm{N}^{\circ}$ 1, Págs. 55-66.

FUENTES-FERNÁNDEZ, R. et al. (2010) "Metamodelling for Agent Based Modelling: An Application for Continuous Double Auctions". In ORTIZ, Á.; R. FRANCO \& P. GASQUET (EDS.). . v. 322, p.285-292, Págs. 285-292. Springer Boston.

GALLO, E. (2011) "Bargaining and social structure". In s.d. Nº February, Págs. 1-46.

GALÁn ORDAX, J. M. (2007) "Evaluación Integradora de Políticas de Agua: Modelado y Simulación con Sociedades Artificiales de Agentes". In Universidad de Burgos.

GALÁN ORDAX, J. M.; A. LÓPEZ PAREDES \& R. DEL OLMO MARTÍNEZ (2005) "Simulación basada en agentes en Teoría de Juegos Evolutiva: ¿pueden las metanormas evitar el colapso de una norma social?". In IX Congreso de Ingeniería de Organización: 65-66. Oviedo: ADINGOR.

GALÁN, J. M. \& L. R. IZQUIERDO (2005) "Appearances Can Be Deceiving: Lessons Learned Re-Implementing Axelrod's "Evolutionary Approach to Norms"". In Journal of Artificial Societies and Social Simulation. Vol. 8, N 3, Págs. 2. JASSS.

GALÁN, J. M. et al. (2009) "Errors and Artefacts in Agent-Based Modelling". In Journal of Artificial Societies and Social Simulation. Vol. 12, $\mathrm{N}^{\circ} 1$,

GALÁN, J. M.; M. M. LATEK \& S. M. M. RIZI (2011) "Axelrod's Metanorm Games on Networks.". In PloS one. Vol. 6, Nº 5, January. Págs. e20474.

GALÁN, J. M.; A. LÓPEZ-PAREDES \& R. DEL OLMO (2009) "An agent-based model for domestic water management in Valladolid metropolitan area". In Water Resources Research. Vol. 45, № 5, Págs. 1-17. American Geophysical Union.

GALÁN, J. M.; R. DEL OLMO \& A. LÓPEZ-PAREDES (2008) "Diffusion of Domestic Water Conservation Technologies in an ABM-GIS Integrated Model". In System. CORCHADO, E.; A. ABRAHAM \& W. PEDRYCZ (EDS.). Vol. 5271, Págs. 567574. Springer Berlin Heidelberg.

GILBERT, N. (2008) Agent-Based Models. LIAO, T. F. (ED.), s.l., Sage Publications. 
GILBERT, N. \& R. CONTE (1995) Artificial Societies: The Computer Simulation of Social Life. GILBERT, N. \& R. CONTE (EDS.), s.l., UCL Press.

GILBERT, N. \& K. G. TROITZSCH (1999) Simulation for the social scientist. s.l., Buckingham: Open University Press.

GIRVAN, M. \& M. E. J. NEWMAN (2002) "Community structure in social and biological networks.". In Proceedings of the National Academy of Sciences of the United States of America. Vol. 99, N²12, June. Págs. 7821-6.

GUIMERÀ, R. et al. (2006) "The real communication network behind the formal chart: Community structure in organizations". In Journal of Economic Behavior \& Organization. Vol. 61, º 4, December. Págs. 653-667.

HARE, M. \& P. DEADMAN (2004) "Further towards a taxonomy of agent-based simulation models in environmental management". In Mathematics and Computers in Simulation. Vol. 64, º 1, Págs. 25-40. Elsevier Science Publishers B. V.

HERNÁNDEZ IGLESIAS, C. \& A. LÓPEZ PAREDES (1999) "Beyond Experimental Economics: Trading Institutions and Multiagent Systems". In Computing in Economics and Finance 1999 from Society for Computational Economics. Págs. 110.

HERNÁNDEZ, C. et al. (2008) "Socially inspired decision making tool: from the Economy to Management Engineering". In Insights in Current Organization Engineering. (EDS), S. ET AL. (ED.). . p.37-39, Págs. 37-39.

HOLME, P. \& B. KIM (2002) "Growing scale-free networks with tunable clustering". In Physical Review E. Vol. 65, N², January. Págs. 2-5.

HOLT, C. A \& A. E. ROTH (2004) "The Nash equilibrium: a perspective.". In Proceedings of the National Academy of Sciences of the United States of America. Vol. 101, N 12, March. Págs. 3999-4002.

IZQUIERDO, L. R. (2008) "Advancing Learning and Evolutionary Game Theory with an Application to Social Dilemmas". In Online.

IZQUIERDO, L. R. \& R. A. HANNEMAN (2006) "Introduction to the Formal Analysis of Social Networks Using Mathematica". In Network. Págs. 60. University of California-Riverside, CA. 
IZQUIERDO, L. R. \& S. S. IZQUIERDO (2008) "Dynamics of the Bush-Mosteller Learning Algorithm in 2x2 Games". In Weber, Elshaw \& Mayer (eds.) Reinforcement Learning. Theory and Applications, Vienna: I-Tech Education and Publishing.

IZQUIERDO, L. R. et al. (2008) "Modelado de sistemas complejos mediante simulación basada en agentes y mediante dinámica de sistemas". In Empiria: Revista de metodología de ciencias sociales. s.d. N 16, Págs. 85-112.

IZQUIERDO, L. R.; S. S. IZQUIERDO \& F. VEGA-REDONDO (2012) "Learning and Evolutionary Game Theory". In Encyclopedia of the Sciences of Learning. SEEL, N. M. (ED.). Springer, New York.

IZQUIERDO, L. R. et al. (2009) "Techniques to Understand Computer Simulations: Markov Chain Analysis". In Journal of Artificial Societies and Social Simulation. Vol. 12, N 1, Págs. 6. Citeseer.

IZQUIERDO, L. et al. (2007) "Transient and asymptotic dynamics of reinforcement learning in games". In Games and Economic Behavior. Vol. 61, N², November. Págs. 259-276.

IZQUIERDO, S. S. \& L. R. IZQUIERDO (2006) "On the Structural Robustness of Evolutionary Models of Cooperation". In Intelligent Data Engineering and Automated Learning - IDEAL 2006. Lecture Notes in Computer Science. CORCHADO, E. et al. (EDS.). . p.172 - 182, Págs. 172 - 182. Berlin Heidelberg: Springe.

IZQUIERDO, S. S. \& L. R. (2011) "Strictly Dominated Strategies in the ReplicatorMutator Dynamics". In Games 2011. Vol. 2(3), Págs. 355-364.

IZQUIERDO, S. S. \& L. R. (2007) "The impact of quality uncertainty without asymmetric information on market efficiency". In Journal of Business Research. Vol. 60, ํ 8, Págs. 858-867.

IZQUIERDO, S. S.; L. R. IZQUIERDO \& N. M. GOTTS (2008) "Reinforcement Learning Dynamics in Social Dilemmas". In Journal of Artificial Societies and Social Simulation. Vol. 11, $\mathrm{N}^{\circ} 2$, 
IZQUIERDO, S. S. et al. (2006) "Market failure caused by quality uncertainty". In Artificial Economics. BECKMANN, M. et al. (EDS.). Vol. 564,, Págs. 203-213. Springer-Verlag.

JANSSEN, M. A. (2003) "Complexity and Ecosystem Management: The Theory and Practice of Multi-Agent Approaches". In Complexity and Ecosystem Management. JANSSEN, M. A. (ED.). Vol. 8, Nº 2, Págs. 63-74. Edward Elgar Publishers.

JOHNSON, P. E. (1999) "Simulation Modeling in Political Science". In American Behavioral Scientist. Vol. 42, N 10, Págs. 1509-1530.

KAHNEMAN, D. (1979) "Prospect Theory". In Econometrica. Vol. 47, N² 2, Págs. 311. JSTOR.

KANDORI, M. (1992) "Social Norms and Community Enforcement". In The Review of Economic Studies. Vol. 59, $\mathrm{N}^{\circ}$ 1, Págs. 63-80. The Review of Economic Studies Ltd.

KIM, J. \& J. T. MAHONEY (2005) "Property rights theory, transaction costs theory, and agency theory: an organizational economics approach to strategic management". In Managerial and Decision Economics. Vol. 26, Nº 4, June. Págs. 223-242.

KUBERA, Y.; P. MATHIEU \& S. PICAULT (2009) "How to Avoid Biases in Reactive Simulations". In Proc of the 7th International Conference on Practical Applications of Agents and MultiAgent Systems PAAMS 2009. . v. 55/2009, p.100109, Págs. 100-109. Springer-Verlag.

LANSING, J. S. (2003) "Complex Adaptive Systems". In Annual review of anthropology. COWAN, G. A. \& G.-C. ROTA (EDS.). Vol. 32, N 1, Págs. 183-204. JSTOR.

LAVIOS, J. J. et al. (2006) "Could TAC be a laboratory for economists?". In Proceedings of the Fifth International Workshop on Practical Applications of Agents and Multi-Agent Systems (IWPAAMS'06). DÍAZ, F.; J. M. CORCHADO \& F. FDEZ-RIVEROLA (EDS.). . p.209-212, Págs. 209-212.

LOZANO, S.; A. ARENAS \& A. SÁNCHEZ (2008) "Community connectivity and heterogeneity: clues and insights on cooperation on social networks". In Journal of Economic Interaction and Coordination. Vol. 3, № 2, Págs. 183-199. 
LOZANO, S.; A. ARENAS \& A. SÁNCHEZ (2008) "Mesoscopic Structure Conditions the Emergence of Cooperation on Social Networks". In PLoS ONE. GALVANI, A. P. (ED.). Vol. 3, N 4, Págs. 9. Public Library of Science.

LÓPEZ PAREDES, A. (2001) "Análisis e Ingeniería de la Instituciones Económicas. Una metodología basada en agentes". In Servicio Editorial de la Universidad del País Vasco.

LÓPEZ PAREDES, A. (2011) "La eficiencia del intercambio. Una aproximación generativa".

LÓPEZ PAREDES, A. \& C. HERNÁNDEZ IGLESIAS (2008) Agent-based Modelling in Natural Resource Management. s.l.,

LÓPEZ PAREDES, A. \& R. DEL OLMO MARTÍNEZ (1998) "The Social Dimension of Economics and Multiagent Systems". In Socially Situated Intelligence: a workshop held at SAB'98:73-79. EDMONDS, B. \& K. DAUTENHAHN (EDS.). University of Zürich.

LÓPEZ-PAREDES, A. (2004) Ingeniería de Sistemas Sociales. Diseño, Modelado y Simulación de Sociedades Artificiales de Agentes. Valladolid, Servicio de Publicaciones de la Universidad de Valladolid.

LÓPEZ-PAREDES, A.; C. HERNÁNDEZ \& J. PAJARES (2004) "Social intelligence or tag reasoning?". In Proceedings of the 2nd International Conference of the European Social Simulation Association (ESSA '04).

LÓPEZ-PAREDES, A. et al. (2008) "Strategic Behaviour, rationality and bargaining power in two-stage games with artificial socially inspired agents". In Computational Intelligence in Decision and Control - Proceedings of the 8th International FLINS Conference. . p.599-604, Págs. 599-604. World Scientific Publishing Co. Pte. Ltd.

LÓPEZ-PAREDES, A.; C. HERNÁNDEZ-IGLESIAS \& J. GUTIÉRREZ PAJARES (2002) "Towards a new experimental socio-economics Complex behaviour in bargaining". In Journal of SocioEconomics. Vol. 31, N 4, Págs. 423-429.

MAHONEY, J. T. (2005) Economic Foundations of Strategy. s.l., SAGE.

MAYNARD-SMITH, J. (1982) "Evolution and Theory of Games". In American Scientist. Vol. 64,, Págs. 41-45. Cambridge University Press. 
MAYNARD-SMITH, J. \& G. R. PRICE (1973) "The Logic of Animal Conflict". In Nature.

MCALLISTER, R. R. J. et al. (2009) "Research impact within the international arid literature: An Australian perspective based on network theory". In Journal of Arid Environments. Vol. 73, № 9, September. Págs. 862-871.

MILGRAM, S. (1967) "The small world problem". In Psychology Today. Vol. 2, $\mathrm{N}^{\circ}$ 1, Págs. 60-67. New York.

MOSS, S. (1999) "Relevance, Realism and Rigour: A Third Way for Social and Economic Research Conventional alternatives". In Págs. 1-24.

MRVAR, A. (s.f.) "Network Analysis using Pajek". In s.d.

NASH, J. F. (1950) "The bargaining problem". In Econometrica: Journal of the Econometric Society.

NASH, J. F. (1953) "Two-Person Cooperative Games". In Econometrica. Vol. 21, № 1, Págs. 128-140. The Econometric Society.

VON NEUMANN, J. \& O. MORGENSTERN (1944) Theory of Games and Economic Behaviour. s.l., Princeton University Press.

NEWMAN, M. E. J. (2003) "The structure and function of complex networks". In SIAM review.

NEWMAN, M. E. J. \& M. GIRVAN (2004) "Finding and evaluating community structure in networks". In Physical Review E. Vol. 69, $\mathrm{N}^{\circ}$ 2, February. Págs. 26113. American Physical Society.

NOWAK, M. A. \& R. M. MAY (1992) "Evolutionary games and spatial chaos". In Nature. Vol. 359, Nº 6398, Págs. 826-829. Nature Publishing Group.

OHTSUKI, H. (2011) "Evolutionary Dynamics of the Nash Demand Game: A Diffusion Approach". In Dynamic Games and Applications. Vol. 1, № 3, April. Págs. 449461. 
PAJARES, J. (2001) "Modelos evolucionistas en dinámica industrial: un enfoque cognitivo". In Tesis Doctoral, Departamento de Organización de Empresas. ETS de Ingenieros. Universidad del $\mathrm{Pa} \backslash \backslash$ is Vasco.

PAJARES, J.; C. HERNÁNDEZ-IGLESIAS \& A. LÓPEZ-PAREDES (2004) "Modelling Learning and R\&D in Innovative Environments: a Cognitive Multi-Agent Approach". In Journal of Artificial Societies and Social Simulation. Vol. 7, № 2,

PAJARES, J.; A. LÓPEZ \& C. HERNÁNDEZ (2003) "Industry as an organisation of agents: Innovation and R\&D management". In JASSS. Vol. 6, N², Págs. 1-20.

PAJARES, J. et al. (2003) "A behavioral, evolutionary and generative framework for modelling financial markets". In Online proceedings of The First European Social Simulation Association Conference, ESSA'03. Groningen.

PASCUAL RUANO, J. A. (2006) "Modelado Multiagente de Mercados Financieros: Un Enfoque Basado en el Comportamiento Individual de los Inversores". In A. López Paredes 6 J. Pajares Gutiérrez (Advisors): Escuela Técnica Superior de Ingenieros Industriales. Universidad de Valladolid.

PASCUAL RUANO, J. A. et al. (2003) "Mercados Financieros Artificiales: un Paso más hacia la Comprensión de los Mercados Financieros Reales". In Actas del $V$ Congreso de Ingeniería de Organización. REDONDO, A. \& J. J. DE BENITO (EDS.). October.

PASCUAL, J. A. \& J. PAJARES (2007) "The role of risk aversion and technical trading in the behaviour of financial markets". In Social Simulation: Technologies, Advances and New Discoveries, Idea Group Inc. EDMONDS, H.-I. AND T. (ED.).

PASCUAL, J. A. et al. (2009) "Una herramienta didáctica para la enseñanza de la teoría de juegos mediante internet". In EDUTEC Revista Electrónica de Tecnología Educativa. Vol. 29, ,

PASCUAL, J. A.; J. PAJARES \& A. LÓPEZ-PAREDES (2006) "Explaining the Statistical Features of the Spanish Stock Market from the Bottom-Up". In BRUUN, C. (ED.). . v. 584, p.283-294, Págs. 283-294. Springer Berlin Heidelberg.

PAVÓN, J. \& J. J. GÓMEZ-SANZ (2003) "Agent Oriented Software Engineering with INGENIAS". In Multi-Agent Systems and Applications III, 3rd International Central and Eastern European Conference on Multi-Agent Systems, CEEMAS. 
MARIK, V., MÜLLER, J. \& PECHOUCEK, M. (ED.). . v. 2691, p.394-403, Págs. 394-403. Springer-Verlag.

PENROSE, E. T. (1959) "The Theory of Growth of the Firm". In Blackwell Oxford. John Wiley.

POLLOCK, G. B. (1989) "Evolutionary stability of reciprocity in a viscous lattice". In Social Networks. Vol. 11, Nº 3, September. Págs. 175-212.

PORTER, M. A.; J.-P. ONNELA \& P. J. MUCHA (2009) "Communities in Networks". In World Wide Web Internet And Web Information Systems. Vol. 56, № 9, Págs. 1082-1097.

PORTER, M. E. (1980) Competitive Strategy: Techniques for Analyzing Industries and Competitors. s.l., Free Press.

POSADA CALVO, M. (2005) "Análisis y diseño de la subasta doble. Una aproximación basada en agentes". In Universidad de Valladolid.

POSADA CALVO, M.; C. HERNÁNDEZ IGLESIAS \& A. LÓPEZ PAREDES (2004) "Aprendizaje Evolutivo en la Subasta Doble Continua. Un enfoque Multiagente". In VIII Congreso de Ingeniería de Organización. . p.111-120, Págs. 111-120.

POSADA CALVO, M.; C. HERNÁNDEZ-IGLESIAS \& A. LOPEZ-PAREDES (2006) "Learning in Continuous Double Auction Market". In Artificial Economics. Págs. $41-51$.

POSADA, M. (2006) "Strategic Software Agents in Continuous Double Auction under Dynamic Environment". In Intelligent Data Engineering and Automated Learning IDEAL 2006. Lecture Notes in Computer Science 4224. . p.1223-1233, Págs. 12231233. Berlin Heidelberg: Springer.

POSADA, M. \& A. LÓPEZ-PAREDES (2008) "How to choose the bidding strategy in continuous double auctions: Imitation versus take-the-best heuristics". In JASSS. Vol. 11, $\mathrm{N}^{\circ} 1$,

POSADA, M.; C. HERNÁNDEZ \& A. LÓPEZ (2008) "Testing Marshallian and Walrasian Instability with an Agent Based Model". In Advances in Complex Systems. . p.249-260, Págs. 249-260. World Scientific Pu . Singapore. 
POSADA, M.; C. HERNÁNDEZ \& A. LÓPEZ-PAREDES (2006) "Strategic Behaviour in Continuous Double Auction". In Lecture Notes in Economics and Mathematical Systems. Págs. 31-43.

POSADA, M.; C. HERNÁNDEZ \& A. LÓPEZ-PAREDES (2007) Emissions Permits Auctions: An Agent Based Model Analysis. EDMONDS, B.; K. G. TROITZSCH \& C. HERNÁNDEZ IGLESIAS (EDS.), s.l., IGI Global.

POZA, D. J. et al. (2011) "Mesoscopic Effects in an Agent-Based Bargaining Model in Regular Lattices". In PLoS ONE. Vol. 6, N 3, March. Págs. e17661. Public Library of Science.

POZA, D. J. et al. (2011) "New Insights on the Emergence of Classes Model". In Discrete Dynamics in Nature and Society. Vol. 2011,, Págs. 1-17.

POZA, D. et al. (2010) "An Agent Based Model of the Nash Demand Game in Regular Lattices". In Balanced Automation Systems for Future Manufacturing Networks. IFIP Advances in Information and Communication Technology. . v. 322/2010, p.243-250, Págs. 243-250.

POZA, D.; F. VILLAFÁÑEZ \& J. PAJARES (2009) "Impact of Tag Recognition in Economic Decisions". In Artificial Economics. HERNÁNDEZ, C.; M. POSADA \& A. LÓPEZ-PAREDES (EDS.). . v. 631, p.245-256, Págs. 245-256. Springer Berlin Heidelberg.

RESNICK, M. (1997) Turtles, termites and traffic jams. s.l., MIT Press.

ROCA, C. P.; J. A. CUESTA \& Á. SÁNCHEZ (2009) "Evolutionary game theory: Temporal and spatial effects beyond replicator dynamics.". In Physics of life reviews. Vol. 6, N 4, August. Págs. 208-249. Elsevier B.V.

ROCA, C. P. et al. (2010) "Topological Traps Control Flow on Real Networks: The Case of Coordination Failures". In PLoS ONE. SPORNS, O. (ED.). Vol. 5, $\mathrm{N}^{\circ} 12$, Págs. 9. Public Library of Science.

ROSS, D. (2011) "Game Theory". In The Stanford Encyclopedia of Philosophy (Fall 2011 Edition). Edward N. Zalta (ed.).

RUBINSTEIN, A. (1998) Modeling Bounded Rationality. s.l., Zeuthen Lecture Book Series. 
RUBINSTEIN, A. (1982) "Perfect Equilibrium in a Bargaining Model". In Econometrica. Vol. 50, ํ1, Págs. 97-109. JSTOR.

SANSORES, C. \& J. PAVON (2005) "Agent-based simulation replication: A model driven architecture approach". In MICAI 2005 Advances in Artificial Intelligence 4 th Mexican International Conference on Artificial Intelligence Monterrey Mexico November 14182005 Proceedings Lecture Notes in Computer Science. GELBUKH, A. F., DE ALBORNOZ, A. \& TERASHIMA-MARÍN, H. (ED.). . v. 3789, p. $244-253$.

SANSORES, C.; J. PAVÓN \& J. GÓMEZ-SANZ (2006) "Visual modeling for complex agent-based simulation systems". In Multi-Agent-Based Simulation VI, International Workshop, MABS 2005, Utrecht, The Netherlands, July 25, 2005, Revised and Invited Papers. Lecture Notes in Computer Science 3891. SICHMAN, J. S. \& ANTUNES, L. (ED.). . p.174-189, Págs. 174-189. Springer-Verlag.

SANTOS MARTÍN, J. I.; R. DEL OLMO MARTÍNEZ \& J. PAJARES GUTIÉRREZ (2005) "Fenómenos de difusión de conocimiento y dimensión geográfica de la innovación, una aproximación multi-agente". In IX Congreso de Ingeniería de Organización.

SANTOS, J. I.; J. M. GALÁN \& R. DEL OLMO (2006) "An Agent-Based Model of Personal Web Communities". In Intelligent Data Engineering and Automated Learning Lecture Notes in Computer Science. CORCHADO, E. et al. (EDS.). . v. 4224, p.1242-1249, Págs. 1242-1249. Springer Berlin Heidelberg.

SANTOS, J. I.; R. OLMO \& J. PAJARES (2007) "The Emergence of Social Networks from Interactive Learning". In S. Takahashi, D. Sallach \& J. Rouchier (Eds), Advancing Social Simulation.The First World Congress, XVI; Berlin Heidelberg: Springer.

SANTOS, J. I. et al. (2012) "Evolution of Equity Norms in Small-World Networks". In Discrete Dynamics in Nature and Society.

SCHELLING, T. C. (1978) Micromotives and Macrobehavior. s.l., Norton.

SCHELLING, T. C. (1971) "Dynamic models of segregation". In Journal of Mathematical Sociology. Vol. 1, N May, Págs. 143-186. Routledge. 
SIMON, H. A. (1957) Models of Man: Social and Rational. s.l., New York: John Wiley and Sons, Inc.

SMITH, V. (1989) "Theory, Experiment and Economics". In Journal of Economic Perspectives. Vol. 3,, Págs. 151-169.

DE SOLA POOL, I. \& M. KOCHEN (1978) "Contacts and influence". In Social Networks. Vol. 1, N²1, Págs. 5-51. Princeton Univ Pr.

SOLÉ, R. (2009) Redes Complejas. Del genoma a Internet. s.l., Tusquets editores.

SULEIMAN, R.; K. G. TROITZSCH \& N. GILBERT (2000) "Tools and techniques for social science simulation". In JasssThe Journal Of Artificial Societies And Social Simulation. Págs. viii, 387. Physica.

SWEDBERG, R. (2001) "Sociology and game theory: Contemporary and historical perspectives". In Theory and Society. Vol. 30, N 3, Págs. 301-335. Springer Netherlands.

TAYLOR, P. D. \& L. E. O. B. JONKER (1978) "Evolutionarily Stable Strategies and Game Dynamics". In Mathematical Biosciences. Vol. 40,, Págs. 145-156.

TESFATSION, L. (2002) "Agent-based computational economics: growing economies from the bottom up.". In Artificial Life. Vol. 8, № 1, Págs. 55-82. MIT Press.

VEGA-REDONDO, F. (2003) Economics and the Theory of Games. s.l., Cambridge University Press.

VEGA-REDONDO, F. (2007) Complex Social Networks. s.l., Cambridge University Press.

VILLAFÁÑEZ, F. \& D. J. POZA (2010) "Propuesta de Modelo MAS para la resolución del RCMPSP basado en Subastas Combinatorias". In Best Practices in Project Management. Methodologies and case studies in Construction and Engineering. GUTIÉRREZ PAJARES, J.; A. LÓPEZ-PAREDES \& C. IGLESIAS HERNÁNDEZ (EDS.).

VILLAHOZ, J.; R. DEL OLMO MARTÍNEZ \& A. ARAUZO (2010) "Combinatorial Auctions for Coordination and Control of Manufacturing MAS: Updating Prices 
Methods". In CORCHADO, E. et al. (EDS.). . v. 73, p.27-30, Págs. 27-30. Springer Berlin / Heidelberg.

VÁZQUEZ BUSTELO, D. (2011) "Proyecto docente e investigador". In Universidad de Oviedo.

WATSOn, J. (2009) "No Title". In The New Palgrave Dictionary of Economics Online. Edited by Steven N. Durlauf \& Lawrence E. Blume.

WATTS, D. J. (1999) "Networks, Dynamics, and the Small-World Phenomenon". In American Journal of Sociology. Vol. 105, N², September. Págs. 493-527.

WATTS, D. J. \& S. H. STROGATZ (1998) "Collective dynamics of "small-world" networks.". In Nature. NEWMAN, M.; A.-L. BARABÁSI \& D. J. WATTS (EDS.). Vol. 393, N 6684, Págs. 440-2. Nature Publishing Group.

WILENSKY, U. (1999) "NetLogo Segregation model". In Center for Connected Learning \& Computer-Based Modeling, Northwestern University, Evanston, IL.

WILLIAMSON, O. E. (2000) "The new institutional economics: taking stock, looking ahead". In Journal of economic literature. Vol. 38, N³, Págs. 595-613. JSTOR.

WOLFE, L. D. (2003) "Dynamics in Human and Primate Societies: Agent-Based Modeling of Social and Spatial Processes". In American Anthropologist. Vol. 105, $\mathrm{N}^{\circ} 4$, December.

WOOLDRIDGE, M. \& N. R. JENNINGS (1995) "Intelligent Agents: Theory and Practice". In October. s.d. N January, Págs. 1-62.

YOUNG, H. P. (1998) Individual Strategy and Social Structure. s.l., Princeton University Press.

YOUNG, H. P. (1993) "An evolutionary model of bargaining". In Journal of Economic Theory. Vol. 59, Nº 1, Págs. 145-168. ACADEMIC PR.

YOUNG, H. P. (2004) Strategic learning and its limits. s.l., Oxford University Press. 

BLOQUE II 

New Insights on the Emergence of Classes Model 



\title{
Research Article
}

\section{New Insights on the Emergence of Classes Model}

\author{
David J. Poza, Félix A. Villafáñez, Javier Pajares, \\ Adolfo López-Paredes, and Cesáreo Hernández
}

Grupo InSiSoc. EII Valladolid, Universidad de Valladolid, Paseo del Cauce S/N, 47011 Valladolid, Spain

Correspondence should be addressed to David J. Poza, djpoza@gmail.com

Received 10 November 2010; Accepted 12 January 2011

Academic Editor: Binggen Zhang

Copyright $\odot 2011$ David J. Poza et al. This is an open access article distributed under the Creative Commons Attribution License, which permits unrestricted use, distribution, and reproduction in any medium, provided the original work is properly cited.

We show the results of a detailed replication of the Emergence of Classes Model Axtell et al. (2004) We study the effect of possible biases on the original proposal and we find additional results and conclusions. We also explore the effects of minor changes on the decision rules that agents play.

\section{Introduction}

The efforts for replicating to replicate previous published models have grown during recent years. However, model replicating is a very tough task, as it was showed by Axelrod [1] and Edmonds [2]. In this paper, we replicate the model by Axtell et al. [3] (hereafter AEY), where two agents want a portion of the same pie, and the portion that a particular agent gets depends on the portion demanded by the other agent. Our results are in agreement with their conclusions, both with nondistinguishable and distinguishable agents (the tag model), as Lopez-Paredes et al. [4] and Dessalles et al. [5] also confirmed in a previous replication of this work.

In this paper, we analyze the hypothesis that researchers should make to obtain the results shown in AEY'S model, and we pay special attention to (a) the initial conditions of the system (potential artefacts/biases following Galán et al. [6] and Kubera et al. [7]), (b) how dependent the results are on the reward values in the payoff matrix, and (c) different ways in which an agent can take a decision. These considerations should be carefully explained to facilitate replication and prevent researchers from making erroneous hypothesis and considering particular cases as general conclusions.

After that, we go one step further by introducing a change in the agents' decision rule: agents behave more realistically and do not compute average benefits. Their decision depends on the most likely option taken by their opponents in previous games. 
Finally, we change the way in which the agents are paired by placing them on a regular spatial structure and forcing them to play against any of their neighbours.

Our results confirm the role that tags play in the emergent behaviour of artificial societies. The effect of tags in human decision processes has been empirically demonstrated by Ito et al. [8].

\section{The Model}

We begin by replicating the bargaining model by AEY. In this model, two players demand some portion of a "pie" (which is a metaphor for a property that is going to be shared out). The portion of pie that they get (i.e., the reward) depends on the other agent's demand. They can demand three different portions of the pie: low, medium or high. As long as the sum of the two demands is not more than 100 percent of the pie, they receive what they demanded; otherwise each gets notting.

There is a population of $n$ agents that play in random pairs. Each agent has a memory in which she maintains the decision taken by their opponents in previous matches. The information collected in their memory is used to demand the portion of the pie that maximizes her expected benefit (with probability $1-\varepsilon$ ), although sometimes, with probability $\varepsilon$, the decisions are taken randomly.

At first, the authors assume that the agents are not distinguishable from one another (except for the content of their memories). They conclude that, whenever there are not observable differences among the agents, there is only one possible state of equilibrium, in which all the pie is shared out among the agents (all the agents learn to compromise and demand "half of the pie"). However, under certain conditions, a "fractious state" can emerge: in this case, all the agents are either aggressive or passive (some of them demand low and some of them demand high), and no equilibrium is reached.

In a second stage of their research, they add a visual "tag" to the agents. The players are capable of identifying their opponent's tag and they store the decision taken by their opponents in a different memory set (depending on the opponent's tag). In this case, the authors prove that, just by adding different tags to the players, discriminatory states can emerge under certain conditions, in which agents with different tags follow different behaviours.

\section{The Model with One Agent Type}

\subsection{Replication}

In our replication of the AEY's model, we used the original payoff matrix (i.e., the combination of rewards for the different demands) 30 percent for low, 50 percent for medium, and 70 percent for high. We also used the original decision rule.

When two players are paired to play, each one gets the portion that she demands as long as the sum of the two demands is less than or equal to 100 percent of the pie. For example,

(i) if player 1 demands 30 , she will receive 30 independently of player 2's decision (when player 1 chooses 30, the sum of 30 (player 1's demand) and all the possible combinations of demands for player 2 are less than or equal to 100 percent of the pie.) 
Discrete Dynamics in Nature and Society

(ii) if player 1 demands 50 , she will get 50 unless player 2 demands 70 (if player 2 chooses 70, the sum of the two demands is higher than 100 percent of the pie. In this case, both players get nothing.)

(iii) if player 1 demands 70 , she will get 70 only if player 2 demands 30 (if player 2 chooses 50 or 70 , the sum of the two demands exceeds 100 percent of the pie and each agent gets nothing.)

\section{Decision Rule}

What makes an agent choose low, medium or high? An agent will check his memory to find how often each option has been chosen by her opponents. Then, she considers that the probability that her current opponent chooses $30(L)$, for example, is equal to the relative appearance of 30 in her memory. In the same way, she calculates how likely it is for the opponent to choose $50(M)$ and $70(H)$. Once the agent knows this information, she estimates the expected benefit for the three possible options as follows:

$$
\begin{aligned}
B(L)= & L \cdot P(\text { opponent choosing } L)+L \cdot P(\text { opponent choosing } M) \\
& +L \cdot P(\text { opponent choosing } H), \\
B(M)= & M \cdot P(\text { opponent choosing } L)+M \cdot P \text { (opponent choosing } M) \\
& +0 \cdot P(\text { opponent choosing } H), \\
B(H)= & H \cdot P(\text { opponent choosing } L)+0 \cdot P(\text { opponent choosing } M) \\
& +O \cdot P(\text { opponent choosing } H),
\end{aligned}
$$

(where $B\left("{ }^{\prime \prime}\right)$ is the mean benefit I get if I choose " $x$ " and $P($ "event") is the probability that "event" occurs).

Notice that this "rational behaviour" takes place with probability $1-\varepsilon$. However, a random decision is taken with probability $\varepsilon$.

A simulation of this replication is shown in Figures 1 and 2. Both simulations were run with the same initial parameters (the same number of agents, the same memory size and the same uncertainty parameter $\varepsilon$ ).

The simplexes shown in Figures 1 and 2 represent the memory state of the agents. The more demands of $L$ an agent keeps in her memory, the closer to the bottom-right vertex she is plotted. Equivalently, if a player's memory contains a considerable amount of $H^{\prime}$ 's, she is placed near the top vertex. Finally, if most of the elements in an agent's memory are $M$ 's, she is plotted close to the bottom-left vertex.

The simplex is split into three different regions, separated by three "decision borders". The top region is dominated by frequent demands of $H$ in previous matches. This is why agents in this region tend to demand $L$ (with probability $1-\varepsilon$ ), as it maximizes their estimated benefit. On the right region, agents are likely to demand $H$ (with probability $1-\varepsilon$ ) because $L$ is the dominant element in their memories. Agents on the left region have often found that their opponents demand $M$; since demanding $M$ maximizes the expected payoff, they are likely to choose $M$ (with probability $1-\varepsilon$ ) in the current iteration.

The three "decision borders" intersect in a point that represents Nash's equilibrium in which agents have the same preference for $I, M$, or $H$. 


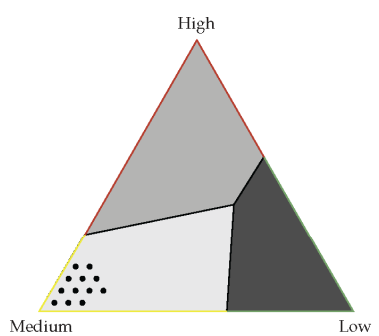

Figure 1: Replication of AEY's model with a number of agents $n=100$, uncertainty parameter $\varepsilon=0.2$, and memory size $m=30$. Equitable equilibrium.

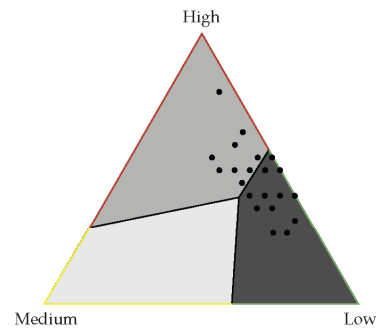

Figure 2: Replication of AEY's model with a number of agents $n=100$, uncertainty parameter $\varepsilon=0.2$ and memory size $m=30$. Fractious state.

AEY states that the system reaches an "equitable equilibrium" when all the agents have, at least, $(1-\varepsilon) \cdot m$ (where $\varepsilon$ is the uncertainty factor and $m$ is the memory size) elements in their memories equal to $M$. Figure 1 shows an equitable equilibrium. In this state, all the agents have found frequent demands of $M$ in the past, and they assume that $M$ is the best response. Because all the agents demand $M$, all the pie is shared out among the players, which means that the system has reached an efficient state. Once the equitable equilibrium is established, it is very difficult for the system to leave this state, (since the system is ergodic, there is still a chance that the system reaches every state in the long term, due to the noise parameter $\varepsilon$.) Figure 2, by contrast, shows a fractious state, in which all the agents are whether aggressive or passive (most of them select $L$ or $H$; $M$ is hardly chosen) and no equilibrium is reached. In this case, the system was started with different random initial conditions. Because the agents have not learnt to compromise, some portions of the pie remains undistributed, which shows the high inefficiency of this system.

Because the system is ergodic, there is a chance that the population evolves from the fractious state shown in Figure 2 to the equitable equilibrium depicted in Figure 1. The number of iterations to achieve this change in the state of the system was defined by AFY as 


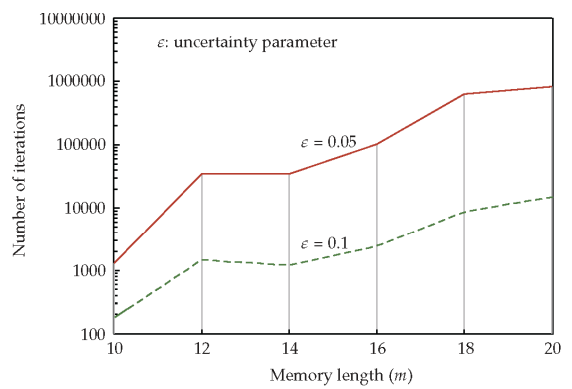

Figure 3: Replication of AEY's model. Transition time as a function of the memory length $(m) ; n=10$; various $\varepsilon$ (uncertainty factor).

"transition time". AEY studied the transition time and analyzed the sensitivity of results to the memory size $(m)$ and the uncertainty factor $(\varepsilon)$, and so we did in our replication. To this aim, we forced the agents' memories so that the system reached a fractious state (Figure 2), and then we measured the number of runs that the system needed to reach the equitable equilibrium (Figure 1). Figure 3 shows the results of our simulation.

Both experiments, the original and the replication, produce the same result in relation with the transition time: it increases as the memory size grows. Notice that this simulation starts in a fractious state; this is why, at first, all the agents tend to demand $L$ or $H$ with high probability $(1-\varepsilon)$ because their memories contain mainly $L$ and $H$. This situation provokes that the agents continue demanding $L$ or $H$ ( $M$ never maximizes their expected benefit (at the first stages of the simulation, when the system is in a fractious state)). Therefore, we depend on the noise parameter $\varepsilon$ to escape the fractious state, as this is the only way to make $M$ appear in the agent's memories, and, consequently, make the agents consider that $M$ is a good option. When the system is started (fractious state), the probabilities that an agent chooses $M$ is $\varepsilon / 3$ (the probability of taking a random decision is equal to $\varepsilon$. Supposing that this is the case, the probability that the random decision is equal to $M$ is one out of three (i.e., the probability that $L$ and $H$ are not randomly chosen). In conclusion, the probability that $M$ is chosen is $\varepsilon / 3$.) This is the reason why the higher $\varepsilon$, the higher the probabilities of leaving the fractious state and thus, the faster the convergence to an equitable equilibrium, as Figure 3 shows.

\subsection{Introduction of a New Decision Rule}

After replicating the original scenario, we changed AEY's decision rule so that the agents demanded the portion of the pie maximizing their benefits against the most likely option taken by their opponents in previous games. In this case, an agent assumes that her opponent's option will be "the mode" of the content of her memory.

An agent will choose $H$ if $L$ is the most frequent decision taken by her opponents in the previous matches; if the most repeated value in her memory is $M$, the player will choose $M$. 


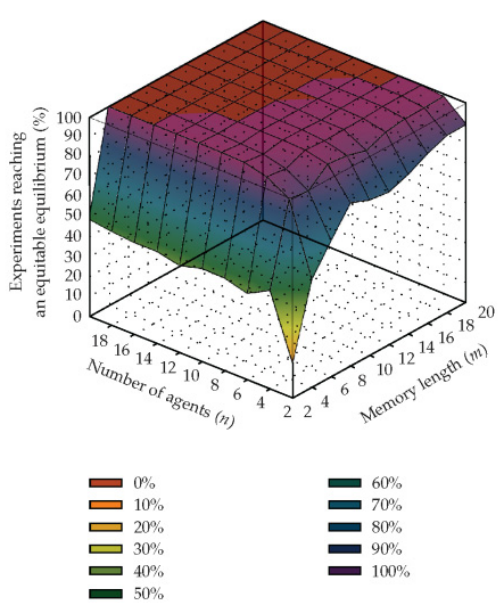

Figure 4: Percentage of experiments that reached a fractious state as the first centre of attraction. Uncertainty parameter $\varepsilon=0.2$. Original decision rule.

If previous matches show that $H$ is the most frequent decision taken by her opponents, she will choose $L$

When the agents used this new decision rule, the chances of reaching the equitable equilibrium in the first place were considerably reduced (as López-Paredes et al. [4] concluded). Figures 4 and 5 show this comparison. To perform this simulation, all the agents where initialized with random memories (as they were in AEY's model), and we measured the percentage of experiments that first reached an equitable equilibrium, versus the number of experiments that first reached a fractious state.

Furthermore, if we only consider the experiments that reached an equitable equilibrium, the time to get it was longer in comparison with the same conditions in the experiment with $\mathrm{AEY}$ 's original decision rule.

Figure 6 shows two simulations of our modification of AEY's model, in which the decision rule has been changed as described before. The left simplex shows an equitable equilibrium and the right simplex displays a fractious state, both after 100 iterations. The simulation was run with the same parameters as in Figures 1 and 2 (100 agents, memory length $=30$ and $\varepsilon=0.2$ ). Notice how the "decision borders" have changed as a result of the introduction of the new decision rule.

\subsection{Payoff Matrix Sensitivity Analysis}

In AEY's model, the values of the possible demands are fixed: 30 percent of the pie for low $(L), 50$ percent of the pie for medium $(M)$, and 70 percent of the pie for high $(H)$. We have 


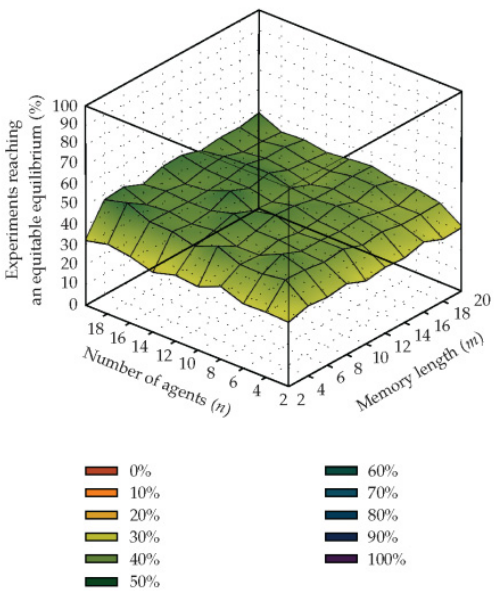

Figure 5: Percentage of experiments that reached an equitable equilibrium as the first centre of attraction. Uncertainty parameter $\varepsilon=0.2$. New decision rule,

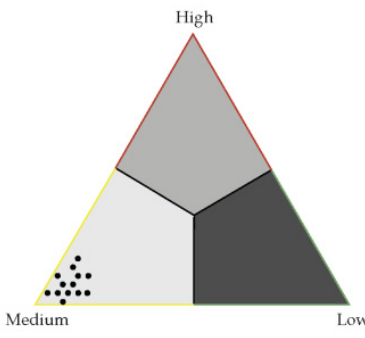

(a)

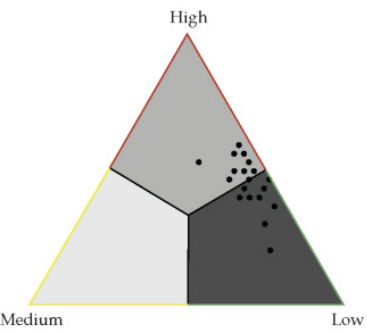

(b)

Figure 6: Modification of AEY's model with a new decision rule. Number of agents $n=100$, uncertainty parameter $\varepsilon=0.2$ and memory size $m=30$. Equitable equilibrium and fractious state.

studied different combinations for the low $(L)$ and high $(H)$ rewards to analyze the effects on the behaviour of the system, (in any case, the sum of the values of $L$ and $H$ is equal to 100 percent of the pie.) The combination of payoffs is shown in Table 1.

The analysis of the simulations showed that when the differences $H-M$ and $M-L$ are high, the transition time between the fractious state and the equitable equilibrium is longer. A comparison of the transition time for different payoff matrices is shown in Figure 7. 


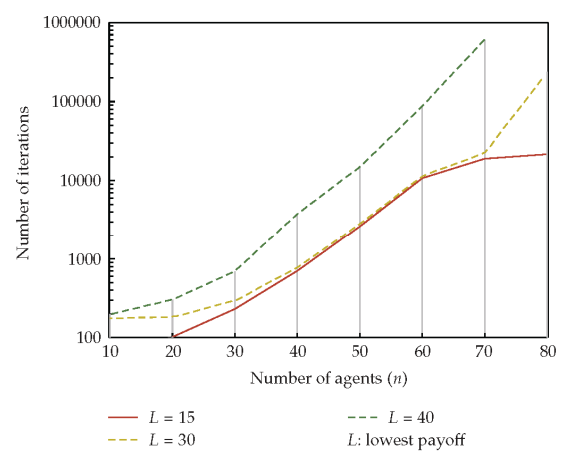

Figure 7: Number of iterations to equitable equilibrium as a function of $L$ (lowest payoff) and $n$ (number of agents); uncertainty parameter $\varepsilon=0.1$ and memory length $m=10$.

Table 1: Possible payoff matrices (combination of demands).

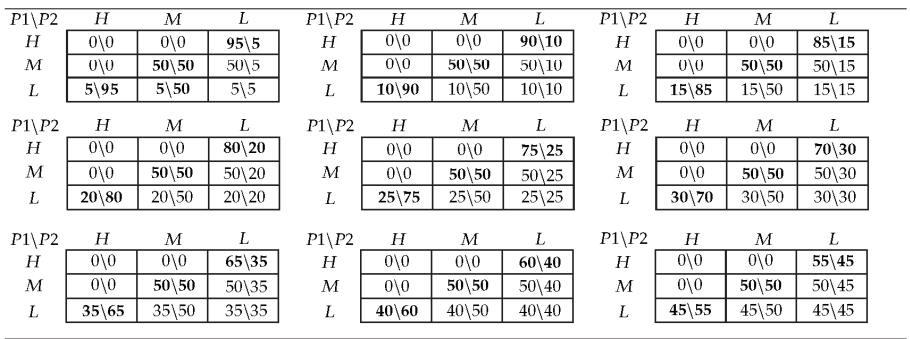

\subsection{Changing the Initial Conditions: "Progressive Memory"}

In AEY's model, all the individuals in the experiment have a fixed-size memory (size $m$ ) along all the matches. The agents are generated with $m$ random values in their memories. Kubera et al. [7] explains that it could introduce biases in the results.

In this modification of the original model, we shall assume that the memory size of each individual grows at a rate of one unity per match, starting with a 0-size memory, until the memory size reaches AEY's fixed value $(m)$. The memory size will not grow any longer when it reaches this value.

To fix ideas, let us suppose that we have defined a memory size of $6(m=6)$. This means that each agent can remember the decision taken by her latest six opponents. Therefore, all the agents have six memory positions. However, in the first match, their 


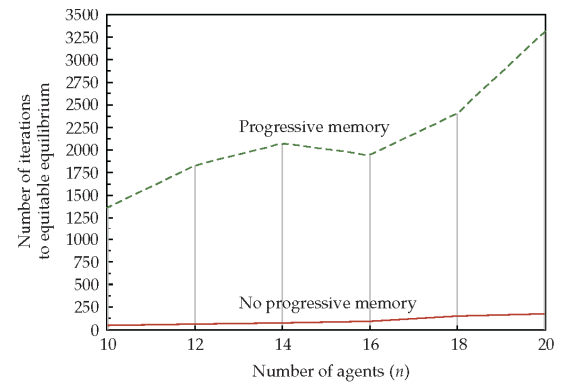

Figure 8: Comparison of AEY's model with and without progressive memory. Number of iterations to equitable equilibrium. Uncertainty parameter $\varepsilon=0.1$. Memory length $m=12$.

memories are empty, as they have never played against any other player before. This is the reason why, in the first match, the decision taken by each agent is random. Afterwards, all the agents store the decision taken by their opponents, as they did in AEY's model. They will use this information to take a decision in the second match, with the same criteria as in AEY's model. Then, the decision taken by their opponents will be stored in their memories once again. In the third match, each agent will have information about the two previous matches; they will take a decision based on this information and store the decision taken by their opponents, and so on. When the number of matches is higher than the memory size for each agent $(m)$, the agents will store the decisions taken by their opponents in their memories, but will eliminate the oldest value in their memories so that the memory size is equal to $m$ in the following matches.

Figure 8 compares the time it takes for the system to reach the equitable equilibrium, both with and without progressive memory. If the system lacks progressive memory (original AEY's model), agent's memories are initialized with $m=12$ random values. In the case of progressive memory, each agent's memory is started with one random value and their memory grows in one element iteration by iteration until it reaches length $m=12$.

The simulation showed that just by changing the initial conditions, the results of the simulation are completely different.

First, as Figure 8 shows, the time it takes for the system to reach the equitable equilibrium is longer than in AEY's original model. Because the first decision is random, the chances of choosing $L$ or $H$ are twice the chances of choosing $M$, which makes the system approach to the fractious state during the first steps of the simulation. The presence of noise in the system $(\varepsilon \neq 0)$, makes it possible that agents choose $M$ with certain probability, which leads the system to the equitable equilibrium in the long term. Because of this transitory situation, in which the system tends to approach the fractious state during some iterations, the number of runs until the system reaches the equitable equilibrium is higher than in AEY's model.

Secondly, notice that, in the case of progressive memory, the value assigned to $\varepsilon$ is crucial. For low values of $\varepsilon$, the system tends to reach a fractious state. The presence of noise makes the agents choose $M$ at some point of the simulation. The increment of the presence of 


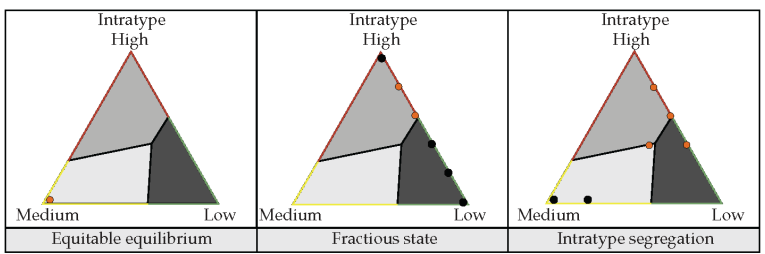

Figure 9: Intratype simplex. Replication of AEY's model with the original decision rule. $n=20$ (10 agents of each type). $m=5 . \varepsilon=0.05$.

$M$ in their memories makes the agents consider that $M$ is a good reply: eventually, the agents learn to compromise and reach an equitable equilibrium. This fact is not likely when $\varepsilon$ grows.

Therefore, although the simulation shows that changing the initial conditions results in an increase of the time to reach the equilibrium, we conclude that initial conditions are irrelevant in the long run.

\section{The Model with Two Agent Types (the "Tag" Model)}

In a second experiment, AEY let the agents be distinguishable from one another by introducing a tag: they create two types of agents, each of whom with a different tag (colour). The agents are capable of identifying their opponents' tag (colour) and they keep the portion of the pie demanded by their opponents in two memory sets, depending on the opponent's tag. AEY states that discrimination (segregation) can emerge spontaneously, both when the agents play with other agents of the same type (intratype matches) and when the agents play against players with different tag (intertype matches).

To study the different cases of segregation, AEY uses two simplexes: one shows the memory state of the agents when they play against agents with their same tag and the other one displays agents' memories when they play against agents with a different tag.

\section{Intratype Segregation}

Figure 9 shows the three scenarios that can arise when players of the same tag play among them (intratype matches).

In the case of intratype matches, we could appreciate three different scenarios.

(i) Equitable equilibrium (all the agents demand $M$ independently of their tag).

(ii) Fractious state (the agents are whether aggressive or passive and do not learn to compromise).

(iii) Intratype segregation: The agents with one tag reach an equitable equilibrium and the agents with the other tag reach a fractious state.

The first and the second scenarios do not show any kind of discrimination: the system reaches an equitable equilibrium or a fractious state independently of the agent's tag, as it did in AEY's model with one agent type. The third scenario is more interesting: when dark players play against dark players, they consider that $M$ is the best response and reach 


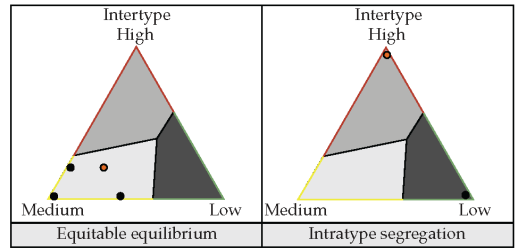

Figure 10: Intertype simplex. Replication of AEY's model with the original decision rule. $n=20$ (10 agents of each type). $m=5 . \varepsilon=0.05$

an equitable equilibrium. However, when light players play among them, they do not learn to compromise and the system reaches a fractious state. This happens even though the decision rule is the same for both types of agents.

\section{Intertype Segregation}

In the case of intertype matches, we can appreciate the two different scenarios shown in Figure 10:

(i) Equitable equilibrium (all the agents demand $M$ independently of their tag).

(ii) Fractious state (the agents of one colour are aggressive-they choose $\mathrm{H}$ - and the agents of another colour are passive-they choose $L$ ).

Some of the experiments showed intertype discrimination. When the agents with different tags are paired to play, the dark agents find that light agents have frequently demanded $H$. Consequently, they decide to choose $L$, which is the only demand that allows them to get a nonzero benefit. On the contrary, after a number of iterations, the light agents have found that light agents are likely to choose low $(L)$. Therefore, they choose high $(H)$, as it maximizes their benefit. This situation can be seen as a "stable fractious state", because the system keeps in this state for longs periods of time: all the agents with one tag are aggressive (they all choose $H$ ) and all the agents of the other tag are passive (all of them choose $L$ ).

After a series of simulations, we conclude that the chances that the system reaches a scenario different from the equitable equilibrium are very low. If fact, when we tried the same parameters that AEY used in their simulation (100 agents, memory size $=20$ ), segregation never emerged, (we contacted Axtell to make sure that we were using the same decision rule that they did.) We needed to reduce the number of agents and the memory length so that we could appreciate segregation (Figures 9 and 10).

Then, we tried changing the decision rule, so that the agents choose the best reply against the most frequent option taken by their opponents in previous matches (mode of their memory), see Section 3.2. The simulation showed that just after changing the decision rule, segregation emerged spontaneously (much more often than when we used the original decision rule). In this case, we easily observed all the possible cases of segregation shown in AF.'s model. 


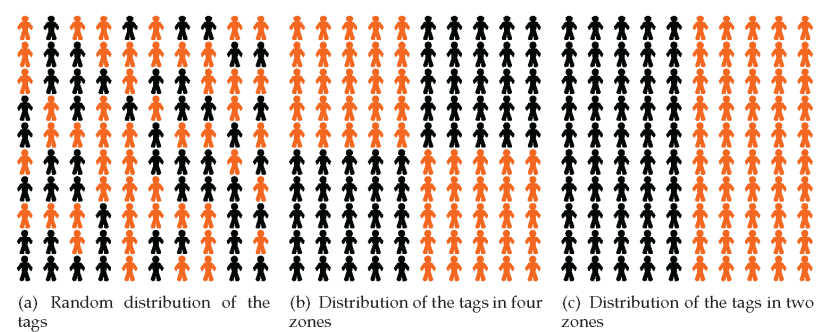

Figure 11: Distribution of the agents in a spatial regular structure. $n=100$ agents ( 50 of each type). Notice that the lattice is a torus

\section{Distribution of the Agents in a Spatial Regular Structure}

In AEY's model, the agents play in random pairs, which means that any agent can play against any other agent of the population. In this new extension, we will consider a $10 \times 10$ toroidal surface where 100 agents will be placed. The agents will be able to play against any of their eight surrounding neighbours (i.e., they can play against any other agent that belongs to her radius $1-$ Moore neighbourhood). As in the original model, there will be the same number of dark-tagged agents and light-tagged agents.

Since the geographical position of each agent is now considered, we will take into account how the tags are distributed in the grid. The effects of the presence of initial clusters have a great relevance in spatial and geographical distribution issues. This is why we will use three different distributions of tags, as Figure 11 shows.

When the agents were randomly paired, we obtained three different results in intratype games (Figure 9) and two different results in intertype games (Figure 10). The aim of this section is to test if these five results can also be obtained when the agents are placed as Figure 11 depicts. To that end, the simulations will be performed with the same parameters that we used in the replication of the original model. We will use the "modebased" decision rule described in Section 3.2, since it facilitates the emergence of segregation in the "tag model", as it was discussed in Section 4.

The simulations confirmed that the same points of attraction that we got in the original model can be obtained with this new extension of the model, both in intratype and intertype games.

Nonetheless, we discovered that, with certain distributions of the tags, it is possible to get new points of attraction that did not appear when the agents were randomly paired to play. This is the case of intertype games when the tags are distributed in two zones (as shown in Figure 11(c)). Figure 12 shows the four points of attraction that the system reaches in the intertype games when this distribution of the tags is used. Notice that due to this distribution of the tags, only the agents that form the borders can play intertype games: in intertype games, each agent is paired with any of their neighbours with different tag.

This distribution of the tags creates two borders between the dark and the light agents (notice that the lattice is a torus). Figure 12(a) shows an equitable equilibrium for both borders (regardless of the agents' tag). In Figure 12(b), all the dark agents demand high 


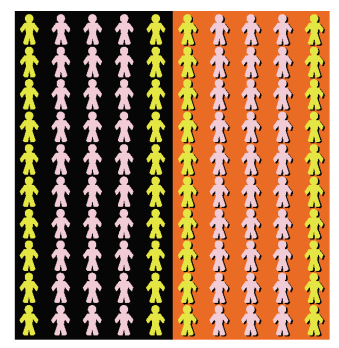

(a) Equitable equilibrium for the two type
of agents. $M-M$ equilibrium in both bo ders

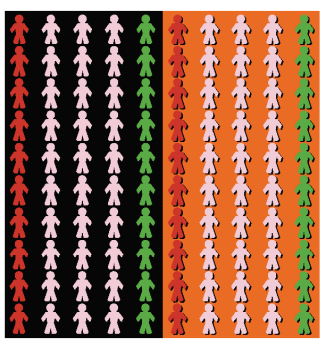

(c) Intertype segregation. $\mathrm{L}-\mathrm{H}$ equilibrium in both borders. The dark and the light agents demand $L$ or $H$ depending on the border

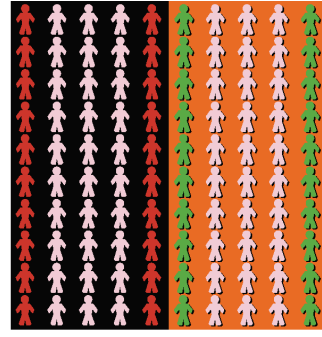

(b) Intertype segregation. $L-H$ equilibrium in both borders. All the dark agents demand $H$; all the light agents demand $L$

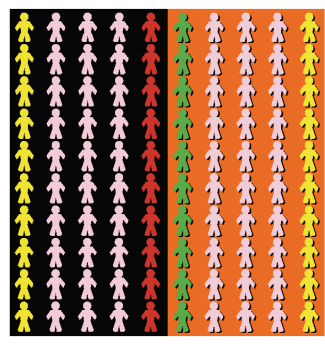

(d) Intertype segregation. $L-H$ or $M-M$ equilibrium depending on the border

Figure 12: Four possible results for intertype matches when the tags are distributed in two zones. $n=100$ agents (50 of each type). Notice that the lattice is a torus.

and all the light agents demand low, which leads to the emergence of segregation. Both borders reach a low-high equilibrium. Figure 12(c) shows a new case of intertype segregation. However, in this case, both types of agents demand low or high depending on which border they are. Finally, Figure 12(d) shows another case of intertype segregation: the agents in one of the borders reach a medium-medium equilibrium and the agents in the other border reach a low-high equilibrium.

Notice that the results shown in Figures 12(a) and 12(b) also appeared in AEY's original model (they are equivalent to the points of attraction shown in Figure 10). By contrast, the results shown in Figures 12(c) and 12(d) only appeared after placing the agents on a grid and distributing the tags in two zones.

However, we conclude that, as there is no connection among the agents that form the two borders, the equilibrium that they reach is independent of one another. Nevertheless, a more in-depth analysis showed that more complex equilibriums can emerge when a set of agents acted as borders between tags and these borders were not connected between them. 


\section{Conclusions}

In AEY's model, segregation emerges spontaneously, even tough all the agents have the same behaviour rule (regardless of their tag). The recognition of the opponent's tag-which a priori does not need to influence on the decisions, as it is an external property-makes the agents "learn" how to behave depending on whether the agent they play against is a same-tag agent or a different-tag agent.

The replication of AEY's no-tags model, showed that there are two centres of attraction in the system: an equitable equilibrium, in which the agents learn to compromise; and a fractious state, in which all the agents are either aggressive or passive and no equilibrium is reached. Because of the ergodicity of the system, there is a possibility that the state of the system switches between these two regimes. We measured the transition time between the two regimes and observed that it rises as the memory size and the number of agents grow, as [3] concluded. The simulation of our replication is completely in agreement with their results.

The modification of AEY's no-tags model showed interesting results. We conclude that simple changes within the original model (using the mode instead of the mean to take a decision), provokes dramatic changes in the studied system. In fact, when we introduced this new decision rule, the chances of reaching an equitable equilibrium in the first place were considerably lower than in AEY's original model.

Moreover, changing the original payoff matrix resulted in a considerable modification in the transition time: the higher the reward assigned to low, the longer it took for the system to reach the equitable equilibrium.

Initializing the agents with a progressive memory instead of using AEY's fixed-size memory showed an interesting scenario: at first, agents tend to be aggressive or passive, but after a number of iterations, they learn to compromise. This makes the system reach an equitable equilibrium in the long run. Therefore, agents' fractious behaviour in the first stages of the simulation results in an increase of the transition time in comparison with AEY's original model. However, we observed that changing the initial conditions does not affect the system in the longer term.

After replicating the tag model, we conclude that our results are in accordance with the original AEY's work. Additionally, we could appreciate that the chances that segregation emerges were really low when we used the original decision rule. After replacing the original decision rule with the mode-based decision rule, segregation emerged much more often. Placing the agents on a regular spatial structure showed that the system could reach the same points of attraction as in the original model, although, initially, no geographical constraints were considered in the original AEY's model. We are currently considering different distribution of the tags in the grid, which makes it possible the emergence of new equilibriums that did not appear in the original model. In future research we will consider different social networks topologies to study how these equilibriums can be affected by the new topologies.

\section{Appendices}

\section{A. Payoff Matrix and Decision Rule in the Replication of AEY's Model.} Payoff Matrix

Using mathematical notation, the payoff matrix shown in Table 1 can be explained as follows: 
Discrete Dynamics in Nature and Society

$n$ : number of agents

$\varepsilon$ : uncertainty parameter

$m$ : memory length of each agent

$S_{i}$ : space of agent $i(i=1, \ldots, n)$ possible strategies

$j$ : possible strategy $\Rightarrow j \in[L, M, H] / M=50, H=100-L, L<H$

( $L$ : select Low, $M$ : select Medium, $H$ : select High).

$\left[v_{1}, v_{2}, \ldots, v_{m}\right]^{i}:$ memory array of agent $i$, which stores the strategies $v_{k} \in[L, M, H]$ chosen by the opponents in the $m$ previous rounds

$[A, B]$ : couple of agent randomly paired ( $n / 2$ randomly pairs by round).

If agent $A$ chooses strategy $i \in S_{A}$, and agent $B$ chooses strategy $j \in S_{B}$, they will receive $[i, j]$ if $(i+j) \leq 100$, and $[0,0]$ if $(i+j)>100$ (see Table 1 , Combination of payoffs).

\section{Decision Rule}

The decision rule used in AEY's model (Section 3.1) is explained with mathematical notation below:

$n_{j}^{A}$ : number of positions with value $j \in[L, M, H]$ in the memory array of agent $A \Rightarrow\left[v_{1}, v_{2}, \ldots, v_{m}\right]^{A}$

$\operatorname{Pr}\left(B_{j}^{A}\right)=n_{j}^{A} / m \Rightarrow$ Probability estimated by the agent $A$ for the possibility that the opponent $B$ selects the strategy $j$ (equivalent to the relative frequency of occurrence of value $j$ in the memory array of the agent $A$ ).

The utility function for agent $A$ when she selects the strategy $i \in S_{i}=[L, M, H]$ is:

$$
\begin{gathered}
U\left(A_{i}\right)=i \cdot \sum_{j \in S_{B}}\left[\operatorname{Pr}\left(B_{j}^{A}\right) \cdot V(i, j)\right] / i \in S_{A} \\
V(i, j)=1 \quad \text { if }(i+j) \leq 100 \\
V(i, j)=0 \quad \text { if }(i+j)>100 .
\end{gathered}
$$

Then, each agent $A$ selects with probability $(1-\varepsilon)$ the strategy $i$ that maximizes her utility function:

$$
\begin{aligned}
& \text { A selects } i \in S_{A}=[L, M, H] / E U\left(A_{i}\right)=\max U\left(A_{i}\right) \\
& \text { and selects a random strategy } i \in S_{A} \text { with probability } \varepsilon .
\end{aligned}
$$

Example A.1.

$$
\begin{aligned}
& n=10 ; m=5 ; \\
& L=30, M=50, H=70 \Rightarrow S_{A}=[L, M, H]=[30,50,70] \text { - space of possible } \\
& \text { strategies for agent } A,
\end{aligned}
$$


16

if $\left[v_{1}, \tau_{2}, \ldots, v_{m}\right]^{A}=[30,30,50,70,30]$ —urrent memory array of agent $A \Rightarrow n_{30}^{A}=$ $3, n_{50}^{A}=1, n_{70}^{A}=1 \Rightarrow \operatorname{Pr}\left(B_{30}^{A}\right)=3 / 5, \operatorname{Pr}\left(B_{50}^{A}\right)=1 / 5, \operatorname{Pr}\left(B_{70}^{A}\right)=1 / 5$

$$
\begin{aligned}
U\left(A_{30}\right) & =30 \cdot \operatorname{Pr}\left(B_{30}^{A}\right) \cdot V(30,30)+30 \cdot \operatorname{Pr}\left(B_{50}^{A}\right) \cdot V(30,50)+30 \cdot \operatorname{Pr}\left(B_{70}^{A}\right) \cdot V(30,70) \\
& =30 \cdot \frac{3}{5} \cdot 1+30 \cdot \frac{1}{5} \cdot 1+30 \cdot \frac{1}{5} \cdot 1=30, \\
U\left(A_{50}\right) & =50 \cdot \operatorname{Pr}\left(B_{30}^{A}\right) \cdot V(50,30)+50 \cdot \operatorname{Pr}\left(B_{50}^{A}\right) \cdot V(50,50)+50 \cdot \operatorname{Pr}\left(B_{70}^{A}\right) \cdot V(50,70) \\
& =50 \cdot \frac{3}{5} \cdot 1+50 \cdot \frac{1}{5} \cdot 1+50 \cdot \frac{1}{5} \cdot 0=40, \\
U\left(A_{70}\right) & =70 \cdot \operatorname{Pr}\left(B_{30}^{A}\right) \cdot V(70,30)+70 \cdot \operatorname{Pr}\left(B_{50}^{A}\right) \cdot V(70,50)+70 \cdot \operatorname{Pr}\left(B_{70}^{A}\right) \cdot V(70,70) \\
& =70 \cdot \frac{3}{5} \cdot 1+70 \cdot \frac{1}{5} \cdot 0+70 \cdot \frac{1}{5} \cdot 0=42 .
\end{aligned}
$$

Agent $A$ selects 70 with probability $(1-\varepsilon)$, as it maximizes her utility function

$$
\operatorname{EU}\left(A_{70}\right)=\max U\left(A_{i}\right)=42,
$$

and selects a random strategy $i \in S_{A}=[30,50,70]$ with probability $\varepsilon$.

\section{B. New Decision Rule for AEY's Model}

Decision Rule

Using mathematical notation, the mode-based decision rule (used in Section 3.2) is explained below:

Each agent $A$ selects, with probability $(1-\varepsilon)$ her strategy $i$ according to the statistical mode $(\mathrm{Mo})$ of her memory array as follows:

$$
\begin{gathered}
\operatorname{Mo}\left[v_{1}, v_{2}, \ldots, v_{m}\right]^{A}=i / \max n_{j}^{A}=n_{i}^{A} \text { for all } j \in S_{A}=[L, M, H] \\
\text { If } \mathrm{Mo}\left[v_{1}, v_{2}, \ldots, v_{m}\right]^{A}=L \Rightarrow A \text { selects strategy } i=H \\
\text { If } \mathrm{Mo}\left[v_{1}, v_{2}, \ldots, v_{m}\right]^{A}=M \Rightarrow A \text { selects strategy } i=M \\
\text { If } \mathrm{Mo}\left[v_{1}, v_{2}, \ldots, v_{m}\right]^{A}=H \Rightarrow A \text { selects strategy } i=L \\
\text { and selects a random strategy } i \in A \text { with probability } \varepsilon .
\end{gathered}
$$

Example B.1.

$$
\begin{aligned}
& n=10 ; m=5 \\
& L=30, M=50, H=70 \Rightarrow S_{A}=[L, M, H]=[30,50,70] \text { — space of possible } \\
& \text { strategies for agent } A
\end{aligned}
$$


if $\left[v_{1}, v_{2}, \ldots, v_{m}\right]^{A}=[30,30,50,70,30]$ —current memory array of agent $A \Rightarrow n_{30}^{A}=$ $3, n_{50}^{A}=1, n_{70}^{A}=1 \Rightarrow \mathrm{Mo}[30,30,50,70,30]=30 \Rightarrow$ Agent $A$ selects 70 with probability $(1-\varepsilon)$, and selects a random strategy $i \in S_{A}=[30,50,70]$ with probability $\varepsilon$.

\section{Acknowledgments}

The authors acknowledge financial support by the Spanish Council for Science and Innovation, SiCoSSys" project, with Grant Reference TIN2008-06464-C03-02, and Junta de Castilla y León with Grants GREX251/2009 and ABACO-VA006A09. They are grateful to Professor Robert Axtell and the anonymous referees at the ESSA 2009 Conference for their valuable comments. Usual disclaims apply.

\section{References}

[1] R. M. Axelrod, "Advancing the art of simulation in the social sciences," in Simulating Social Phenomena, R. Conte, R. Hegselmann, and P. Terna, Eds., pp. 21-40, Springer, Berlin, Germany, 1927.

[2] B. Edmonds, "Replication, replication and replication: some hard lessons from model alignment," Journal of Artificial Societies and Social Simulation, vol. 6, no. 4, 2003.

[3] R. L. Axtell, J. M. Epstein, and H. P. Young, "The emergence of economic classes in an agent-based bargaining model," in Social Dynamics, S. N. Durlauf and H. P. Young, Eds., pp. 191-211, MIT Press, Cambridge, Mass, USA, 2004.

[4] A. López-Paredes, C. Hernández, and J. Pajares, "Social intelligence or tag reasoning?" in Proceedings of the 2nd International Conference of the European Social Simulation Association (ESSA'04), September 2004, http://www.unikoblenz.de/ essa/ESSA2004/ files/papers/LopezHernandezPajaresESSA04.pdf.

[5] J. L. Dessalles, J. P. Müller, and D. Phan, "Emergence in multi-agent systems: conceptual and methodological issues," in Agent-Based Modelling and Simulation in the Social and Human Sciences, F. Amblard and D. Phan, Eds., vol. 14, pp. 327-355, The Bardwell Press, Oxford, UK, 2007.

[6] J. M. Galán, L. R. Izquierdo, S. S. Izquierdo et al., "Errors and artefacts in agent-based modelling," Journal of Artificial Societies and Social Simulation, vol. 12, no. 1, 2009.

[7] Y. Kubera, P. Mathieu, and S. Picault, "How to avoid biases in reactive dimulations," in Proceedings of the 7 th International Conference on Practical Applications of Agents and Multi-Agent Systems (PAAMS '09), 2009 .

[8] A. Ito, E. C. Willadsen-Jensen, and J. Correll, "Social neuroscience and social perception: new perspectives on categorization, prejudice, and stereotyping," in Social Neuroscience: Integrating Biological and Psychological Explanations of Social Behavior, E. Harmon-Jones and P. Winkielman, Eds., pp. 401-421, Guilford, New York, NY, USA, 2007. 

Mesoscopic Effects in an Agent-Based Bargaining Model in Regular Lattices 



\title{
Mesoscopic Effects in an Agent-Based Bargaining Model in Regular Lattices
}

David J. Poza ${ }^{1 *}$, José I. Santos ${ }^{2}$, José M. Galán ${ }^{2}$, Adolfo López-Paredes'

1 Social Systems Engineering Centre INSISOC, Valladolid, Spain, 2 Área de Organización de Empresas, Departamento de Ingeniería Civil, Universidad de Burgos, Burgos, Spain

\begin{abstract}
The effect of spatial structure has been proved very relevant in repeated games. In this work we propose an agent based model where a fixed finite population of tagged agents play iteratively the Nash demand game in a regular lattice. The model extends the multiagent bargaining model by Axtell, Epstein and Young [1] modifying the assumption of global interaction. Each agent is endowed with a memory and plays the best reply against the opponent's most frequent demand. We focus our analysis on the transient dynamics of the system, studying by computer simulation the set of states in which the system spends a considerable fraction of the time. The results show that all the possible persistent regimes in the global interaction model can also be observed in this spatial version. We also find that the mesoscopic properties of the interaction networks that the spatial distribution induces in the model have a significant impact on the diffusion of strategies, and can lead to new persistent regimes different from those found in previous research. In particular, community structure in the intratype interaction networks may cause that communities reach different persistent regimes as a consequence of the hindering diffusion effect of fluctuating agents at their borders.
\end{abstract}

Citation: Poza DJ, Santos Jl, Galán JM, López-Paredes A (2011) Mesoscopic Effects in an Agent-Based Bargaining Model in Regular Lattices. PLoS ONE 6(3): e17661. doi:10.1371/journal.pone.0017661

Editor: Matjaz Perc, University of Maribor, Slovenia

Received November 22, 2010; Accepted February 5, 2011; Published March 9, 2011

Copyright: $\odot 2011$ Poza et al. This is an open-access article distributed under the terms of the Creative Commons Attribution License, which permits unrestricted use, distribution, and reproduction in any medium, provided the original author and source are credited

Funding: This work stems from the participation of the authors in research projects funded by the Spanish Ministry of Science and Innovation, references TIN2008-06464-C03-02 and CSD2010-00034 (CONSOLIDER-INGENIO 2010), and by the Junta de Castilla y León, references VA006A009, BU034A08 and GREX2512009. The funders had no role in study design, data collection and analysis, decision to publish, or preparation of the manuscript.

Competing Interests: The authors have declared that no competing interests exist.

*E-mail: djpoza@gmail.com

\section{Introduction}

The role that norms play as regulator mechanisms of certain aspects of social, economic and organizational behaviours has been thoroughly studied in the social sciences $[2,3]$. Once a norm has been established, it acts as a self-reinforcement mechanism of behaviour. However, the emergence, diffusion and collapse of social norms are, in general, exempt from explicit mechanisms of control.

There are different kinds of norms depending on the type of social interaction. Concretely, in the economics field, an important research effort is focused on understanding the emergence of norms that determine the property distribution in a community. Thus, in contrast to the equity norm that leads to "distributive justice" and fair division of goods in some communities, there is also evidence of systematic persistence of discriminatory norm that allocate different shares of a resource according to some individual characteristic or group membership.

Evolutionary game theory is a powerful framework to analyse this type of norms formally. In particular, the Nash bargaining game [4] is often used as a simple archetypical model of economic interaction and good distribution. Succinctly, the two-player Nash bargaining game consists of two players that have to divide a sum of money among them. The payoff for each player is the amount of money they asked for, but if the sum of the demands exceeds the total, they both obtain nothing.

If the game is played repeatedly among an infinite population of players that are randomly paired up and change their strategy according to the replicator dynamics then, given a particular initial condition, it is possible to compute the distribution of strategies in the population over time [5]. Notwithstanding, the influence of such assumptions has proved very relevant for the results of evolutionary game models [6]. This is particularly relevant given that such assumptions are not always easy to justify.

In 2001, Axtell, Epstein and Young [1] proposed an agentbased model (henceforth AEY's model) to understand the transient and the asymptotic dynamics of the Nash demand game in a finite population. They simplified the analysis considering just three possible demands: low $(\mathrm{L})$, medium $(\mathrm{M})$ and high $(\mathrm{H})$. They proved that different self-reinforcing norms can emerge spontaneously. These emergent norms may be completely different from one another even though all the agents of the population have exactly the same behavioural rule. Which particular norm appears first depends on initial conditions and on purely accidental events, such as the specific pair of agents that happened to be (randomly) paired at a certain time.

To obtain these conclusions Axtell et al. conduct their analysis in two parts. Initially they study the dynamics of a population of indistinguishable agents with the capacity to store in their memories the strategies played by their opponents in the last encounters. Each agent uses this information to form an expectation about her opponent's strategy, assuming that the probability of the next demand equals the relative frequency of the remembered experiences in the last encounters. Given that belief, each agent responds with a "noisy best reply", i.e. a best reply with a small probability of selecting a random demand. One of the 
norms that can emerge in this setting is the so-called "equity norm", i.e. a self-fulfilling situation where everyone expects the others to demand $\mathrm{M}$ and, as a consequence, everyone demands $\mathrm{M}$ this behaviour, in turn, confirms the expectations that everyone already has, thus closing a self-consistent loop. Axtell et al. point out that the "equity norm" is the unique stochastically stable state of the game (see Young $[7,8]$ for a comprehensive analysis of the required conditions to obtain this conclusion), but they also find other persistent stable fractious states, in which players play repeatedly $\mathrm{L}$ or $\mathrm{H}$, but never $\mathrm{M}$.

More interestingly, in the second part of their analysis, Axtell et al. endow each individual agent with one of two possible tags (which can be recognised by all, but has no initial meaning) and with the ability to remember both the past behaviour of her opponents and their tag. In this second setting, they find that a new stable state can endogenously emerge, in addition to the ones previously observed. In this new state, agents behave differently within and outside their own tag group, so the state was naturally labelled "segregation". The implications of Axtell et al.'s finding are astonishing: a discriminatory norm in which property is unequally distributed based on observable characteristics that are initially meaningless, may not only emerge but even perpetuate for long, as a consequence of the self-reinforcing nature of the dynamics. These results are very suggestive from a social point of view when we associate the concept of tag in the model with some social or cultural trait such as race, gender or age, which may condition people's behaviour in human societies. Using the model as reference, the emergence of a rich variety of collective outcomes can be explained. An example would be the situation where a divided underclass is oppressed by a unified elite: this would correspond in the model to a state where the elite group systematically plays $\mathrm{H}$ against the oppressed group (who respond optimally plaving $\mathrm{L}$ ) and plays equitable $(\mathrm{M})$ among themselves, while the discriminated group is stuck in a fractious state. The replicator dynamics embeds two important assumptions: infinite populations (which is the hypothesis relaxed by Axtell et al. [1]) and random pairings. The assumption that pairings are random can be understood as an abstraction of persistent bargaining interaction with strangers. However, in some contexts this may be unrealistic; agents may interact only with just a small number of other agents with which they are in direct contact $[9,10]$. In those cases the global interaction assumption can be removed and we can analyze the effect of a given social or spatial structure.

Introducing structure in the population implies that the probability of interaction between two agents depends on the specific pair of agents. The structure of the population can be usefully represented by means of a graph or network that describes the interaction connectivity. Ohtsuki et al. [11,12] argue that in a general case, the structure should be described by two graphs, one representing the interaction of the game played and a second one representing the interaction of the adoption or learning mechanism. Usually both graphs are considered the same. The effect of many different types of graphs in games has been investigated, examples of which include the analysis of iterated $2 \times 2$ games such as the Prisoner's Dilemma on regular lattices [13-15], ErdosRenyi [15-17], small-world [15,18], scale-free [16,17,19-23] or real networks [24,25], the analysis of the snowdrift game on lattices [26,27], small-world $[28,29]$ or scale-free [19-21] networks, and n-person games such as public good games on lattices [30-33] or the minority game on small world networks $[34,35]$ (some reviews can be found at $[6,36,37])$. In this article we have extended the analysis of norm diffusion in a population considering AEY's model as a framework. We have studied the influence of the topology on the results of the game. To this aim, we have considered the spatial dimension of the game by introducing a regular spatial structure. We have also modified the original model by adding a new behavioural rule that requires less cognitive abilities than those required in the original paper. When agents use this behavioural rule, the segregation norm emerges more frequently, and a richer space of solutions is observed.

This work is organized as follows: first, we briefly explain the extensions and modifications that we have performed on AEY's original model. Next, we describe the results that we have obtained when agents are randomly assigned a tag. At the end of this section we discuss some cases where several persistent regimes can simultaneously emerge, and their relation with some mesoscopic topological properties. We then finish with the conclusions of this work.

\section{Methods}

Agent-based Model of Bargaining in a Regular Lattice

In this section we describe an agent-based model of bargaining in regular lattices based on the original tag model proposed by Axtell et al. [1]. Our model introduces a spatial restriction in the structure of interactions: agents are embedded on a regular lattice and they can only bargain with their spatial neighbours. In each time period of the model, each agent selects one of her neighbours at random to play the Nash demand game. When playing the game, each agent considers three possible demands of a pie (which is a metaphor of something that is going to be shared between two persons), i.e. low $(L)$ or $30 \%$, medium $(M)$ or $50 \%$ and high $(H)$ or $70 \%$. The agents get the chosen demand if the sum of their demands does not exceed 100 percent of the pie; otherwise they both get nothing (see the payoff matrix in the Table 1). The Nash demand game represented in Table 1 has exactly three purestrategy Nash equilibria, corresponding to the pairs (L, H), (M, M), and $(\mathrm{H}, \mathrm{L})$. These are called the equilibria of the one-shot bargaining game.

As in Axtell et al. [1], the population of agents is divided into two groups of equal size whose members share a recognizable characteristic which has no a priori social meaning, i.e. a tag. These tags are used by the agents to select their demand in the one-shot game. To be precise, the agents' decision rule, which is identical for all individuals, is based on the agents' capacity to remember their opponent's demand in the $m$ most recent interactions with opponents with the same tag (i.e. intratype interaction) and the $m$ most recent interactions with opponents with the other tag (i.e. intertype interaction). These experiences constitute the agent's intratype and intertype memories. In the AEY model, an individual chooses the best reply that maximizes the expected demand considering their past experiences with similar opponents, i.e. those with the same tag. In contrast, in our model we consider a simpler decision rule, henceforth the mode rule, which dictates that individuals choose the best reply against the most frequent demand with similar opponents (ties are

Table 1. Payoff matrix of the Nash demand game.

\begin{tabular}{llll}
\hline & & & \\
\hline & $\mathbf{H}$ & $\mathbf{M}$ & $\mathbf{L}$ \\
\hline$H$ & $(0,0)$ & $(0,0)$ & $\mathbf{( 7 0 , 3 0 )}$ \\
$\mathbf{M}$ & $(0,0)$ & $\mathbf{( 5 0 , 5 0 )}$ & $(50,30)$ \\
$\mathrm{L}$ & $\mathbf{( 3 0 , 7 0 )}$ & $(30,50)$ & $(30,30)$ \\
\hline
\end{tabular}

doi:10.1371/journal.pone.0017661.t001 
Mesoscopic Effects: ABM Model in Regular Lattices

resolved randomly without any bias). The mode rule is cognitively less demanding than AEY's and, naturally, it induces different results than those obtained with the original rule [38].

The stochastic version of the game considers that agents may make mistakes in their decisions (or simply experiment from time to time). Hence, with probability $(1-\varepsilon)$ an individual chooses the best reply and with probability $\varepsilon$ she chooses one of the three possible demands at random (low, medium or high with the same probability).

The model has been implemented in Netlogo (http://ccl. northwestern.edu/netlogo/) and can be downloaded at this url: http://ingor.ubu.es/models/aeygrid).

We can summarize the model as follows: there is a population of $\mathcal{N}$ agents randomly distributed in a regular 2-dimensional toroidal lattice of $L x L=\mathcal{N}$ cells, each one inhabited by one and only one of the $\mathcal{N}$ agents. The population is divided exactly into two groups whose members have a distinctive tag. The number of agents is chosen satisfying simultaneously two conditions: (1) it is even, so the population can be divided exactly into two groups, (2) and it square root is an integer, so the regular lattice is square too. Each agent is endowed with two memories of length $m$ to keep the demands of the two classes of tags. Memories are initialized at random. In each time period $t$, each agent randomly selects one of her 8 neighbours (radius-1 Moore neighbourhood) to play the game. The agent observes her opponent's tag and decides the best reply against the most frequent demand in her corresponding memory (i.e. intratype or intertype). However, with a small probability $\varepsilon$ an agent decides randomly between the three possible demands. Afterwards both agents update their memories. Figure 1 shows the different interaction networks of a particular spatial distribution of tagged agents in a $4 \times 4$ lattice.

\section{Results}

Understanding the dynamics of the model

Before our computational exploration of the model, we have conducted a brief analysis within the framework of Markov Chains [39] to gain some insights about the expected dynamics and behaviour of the model. Fortunately, some aspects of this formal analysis have already been carried out for the AEY model by Axtell et al [1], and for the evolutionary model of bargaining by Young $[7,8]$. In terms of markovian properties, our model shares many characteristics with these models. To represent the model as a time-homogeneous Markov Chain (THMC), we define the state
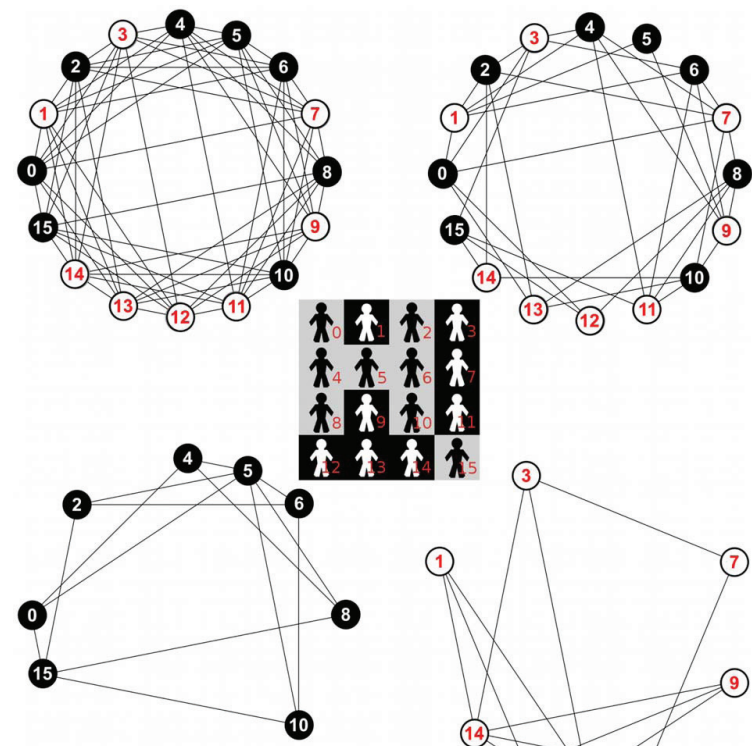

(13) (12) (11)

然
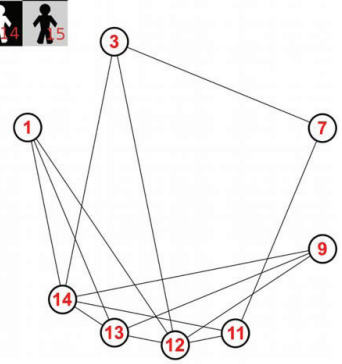

Figure 1. Example of a spatial distribution and its corresponding interaction networks. The spatial distribution, depicted in the centre, consists of 16 agents randomly distributed in a regular 2-dimensional toroidal lattice of $4 \times 4$ cells. The corresponding set of interaction networks are shown in the corners: (i) the complete interaction network (upper left corner), (ii) the intertype interaction network (upper right corner), (iii) the "black tag" intratype interaction network (bottom left corner), and (iv) the "white tag" intratype interaction network (bottom right corner). doi:10.1371/journal.pone.0017661.g001

PLoS ONE I www.plosone.org 
of the system in a time period $\mathrm{t}$ as a $\mathrm{N}$-dimensional vector $\mathrm{X}_{\mathrm{t}}=\left\{\mathrm{X}^{1}, \mathrm{X}^{2}, \ldots, \mathrm{X}_{\mathrm{t}}^{\mathrm{N}}\right\}$ of $2 \mathrm{~m}$-tuples $\mathrm{X}_{\mathrm{t}}^{\mathrm{i}}$, each one corresponding to agent i's memory of both intratype and intertype encounters (it is not necessary to use all $\mathrm{m}$ values of the agents' memory to represent the state space, since knowing only the memory length and two of the frequencies of each possible demand $\{\mathrm{L}, \mathrm{M}, \mathrm{H}\}$ is enough). Note that the spatial distribution of tags conditions the chances of intratype/intertype encounters in each period, but the possible changes that may occur in each interaction are only dependent on the particular form of the two m-tuple memories that are involved in the interaction.

The characteristics of the system dynamics are strongly determined by the presence or absence of errors (mutations in evolutionary terminology) in agents' decisions. In the absence of decision errors, i.e. the unperturbed model, the system has absorbing states in which sooner or later it will be trapped (if we run the model for long enough). These absorbing states are directly related with the three pure-strategy Nash equilibria of the Nash demand game, giving rise to the equity norm $(\mathrm{EQ})$ and the inequity norm $(\mathrm{IQ})$ The former happens when everyone in the population expects the others will demand $\mathrm{M}$, and consequently everyone demands $\mathrm{M}$, so the system ends reaching an absorbing state for both intratype and intertype bargaining processes, which is equitable because al agents get equal payoffs, and is also efficient (in Pareto sense) because no agent can be made better off without making another agent worse off. Apart from this, in the AEY model without spatial restrictions [1], there are also IQ absorbing states for the intertype bargaining game. An IQ equilibrium corresponds to a state in which tagged agents coordinate in one of the two asymmetric pure-strategy Nash equilibria. Whenever agents of one tag expect the others will demand $\mathrm{L}$ and hence they will demand $\mathrm{H}$, and simultaneously the others will expect and demand the comple-

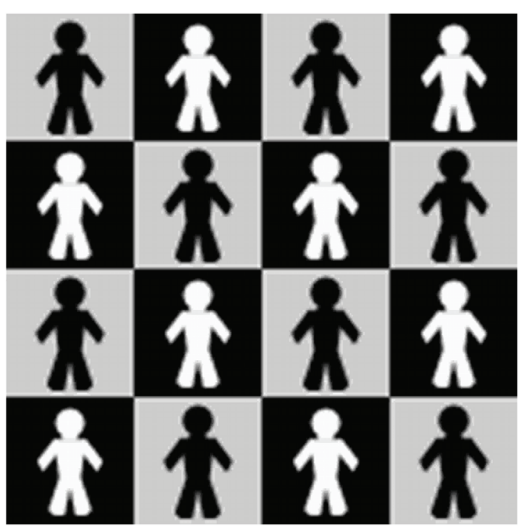

Figure 2. Toroidal grid of $4 \times 4$ cells with 8 white and 8 black Figure 2. Toroidal grid of $4 \times 4$ cells with 8 white and 8 black
agents distributed in the way shown. In this particular spatial agents distributed in the way shown. In this particular spatia distribution the system may reach the IQ state in both intratype game whenever similar tagged agents placed in the same column demand exactly the complementary quantity $(\mathrm{L}$ or $\mathrm{H})$ of their neighbours of columns just next to them. This result is true if the intratype network is bipartite, i.e. there are no odd-length cycles.

doi:10.1371/journal.pone.0017661.g002

A. PLOS ONE I www.plosone.org mentary decisions, the system reaches an absorbing state, which in this case is efficient but not equitable in the proportions obtained by each agent.

Interestingly, additional absorbing states show up as a consequence of the imposed spatial structure. For example, a spatial distribution of $4 \times 4$ tagged agents like the one depicted in Figure 2 allows an IQ absorbing state in both intratype bargaining games, i.e black-black and white-white.

When randomness is introduced in agents' decisions (motivated by the possibility of mistakes or by a simple desire for exploration), the system becomes ergodic. In this case, there is a unique limiting distribution over the state space which determines the probability of finding the system in each of its states in the long run (e.g. $\lim _{t \rightarrow \infty} \mathrm{P}\left(\mathrm{X}_{\mathrm{t}}=\mathrm{i}\right)$ ). Such probabilities are strictly positive and independent of the initial conditions. This limiting distribution can be estimated sampling just one simulation run for a sufficiently long time, by computing the fraction of the time that the system spends in each state, i.e. the occupancy distribution [39]. In contrast to what one may expect, when the tagged model for a finite population and global interaction is asymptotically analysed, this limiting distribution concentrates only on one of the two absorbing states of the unperturbed model, the EQ. The formal demonstration of this relies on the concept of stochastic stability [8]. When some small noise exists, the EQ state is stochastically stable while IQs are not. This implies that, in the long run and for sufficiently unlikely perturbations, the system tends to spend most of the time at the EQ state. Nevertheless, Axtell et al. [1] make an interesting contribution turning the attention from the asymptotic to the transient dynamics, and showing that there are other relevant states in which the system spends a considerable fraction of the time, henceforth persistent regimes. In the transient evolution of the global interaction model, sometimes the system is temporarily trapped in a particular regime, called fractious regime $(\mathrm{FR})$, in which agents alternate their demands between $\mathrm{H}$ and $\mathrm{L}$, making the emergence of the equity norm very difficult (We keep the word fractious for consistency with the original AEY's model; but it may be worth noting that other names, such as "fluctuating agents" $[16,40]$, have been used in the literature for essentially the same concept, i.e. agents that intermittently change their strategy). Moreover, they show that the transition time between this fractious regime FR to the stochastically stable state EQ can be enormously long and this time grows exponentially with the number of agents and their memory length -i.e in their terminology: ergodicity is broken.

Formally, the system is completely characterised by the vector $\mathrm{X}_{\mathrm{t}}$, which can be graphically represented using a 2 -simplex of the agents' states (see Figure 3). Each of the two agent i's memories keeps track of the demands made by her opponents in the $\mathrm{m}$ most recent intratype (or intertype) encounters, and can be represented by a vector of the relative frequencies of these demands $\mathrm{Xi}=\left\{\mathrm{n}_{\mathrm{L}}\right\}$ $\left.\mathrm{m}, \mathrm{n}_{\mathrm{M}} / \mathrm{m}, \mathrm{n}_{\mathrm{H}} / \mathrm{m}\right\}$, where $\mathrm{n}_{\mathrm{L}}$ denotes the number of times that agent i's opponent demanded $\mathrm{L}$ in the $\mathrm{m}$ most recent intratype (or intertype) interactions. This vector corresponds in the simplex with the point $\left(n_{L} / m+n_{H} / 2 m, \sqrt{3} n_{H} / 2 m\right)$. Since the memory of an agent is made by two partitions, corresponding to the past demands of the two classes of opponents, we can use two separated simplexes to represent each one.

\section{Most Frequent Persistent Regimes}

If, following the approach in Axtell et al [1], we focus our analysis on the transient dynamics of the spatial model, the complexity of the system makes us to resort to computer simulation as methodology. We have designed a set of experiments 


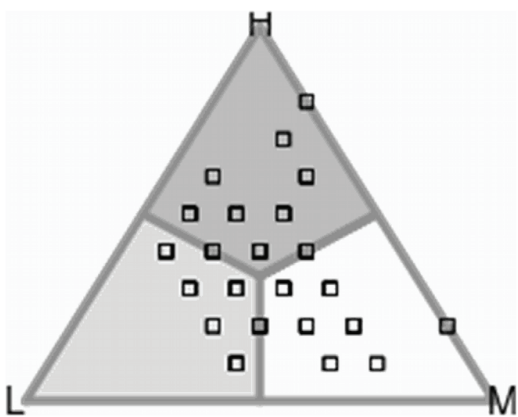

Figure 3. 2-simplex representation of the state space used in both intratype and intertype bargaining games. The shaded regions correspond to the state subspaces in which an agent always decides one of the three possible demands $\{L, M, H\}$. For example, the light grey area at the bottom-left of the triangle represents a set of states in which the majority of the items in the memory are L, and therfore the agent will demand $\mathrm{H}$. The opposite happens in the dark grey area at the top. Finally the $\mathrm{M}$ demand dominates the agents memories in the white area, so the response of the agent in that area is also M. Note that with the mode-decision rule, the centre of the triangle, which is equidistant from the three vertices, corresponds to the indifferent state in which any of the three demands is equally possible.

doi:10.1371/journal.pone.0017661.g003

to discover and understand the persistent regimes that emerge in the model.

The parameterization of all cases mentioned in this paper corresponds to a model of $\mathrm{N}=100$ agents randomly distributed in a regular lattice of 2-dimensional grid of $10 \times 10$ cells, each one keeping one of the $\mathrm{N}$ agents. Each agent is endowed with two memories of length 10 for intratype and intertype bargaining games, initialized at random. In each time period t, each agent selects one of her 8 neighbours (Moore neighbourhood) at random and decides the best reply against the most frequent demand in her memory for the type of opponent. However, with a small probability $\varepsilon=0.01$ an agent decides randomly between the three possible demands $\{\mathrm{L}, \mathrm{M}, \mathrm{H}\}$. Note that each time period consists of $\mathrm{N}$ matches, and consequently it is probable that an agent bargains more than once in each time period. We have sampled 10.000 simulation runs during $\mathrm{T}_{\mathrm{f}}=30.000$ time periods.

The system state at the end of the simulation time can be summarized as a 3-tuple of the regimes reached by the intertype and the two intratype bargaining games \{Intra-white ${ }_{\text {regime }}$, Intrablack $_{\text {regime, }}$ Inter regime $_{\text {re }}$. Taking into account the characterization of the types of stable and persistent regimes described in the previous section, we may expect that if we let the system run for long enough, it will reach one of the $3^{3}$ possible combinations, i.e. \{EQEQEQ\}, \{EQEQFR\}, .... We define a set of simple conditions, henceforth C1 stop conditions, for reaching each of the persistent regimes according to their nature: the EQ state is considered reached whenever all agents in the corresponding bargaining process have at least $(1-\varepsilon) \times m$ instances of $M$ in their memories (note that the memory vector has a finite number of instances, so we approximate $(1-\varepsilon) \times \mathrm{m}$ to the lower integer and $\varepsilon \times \mathrm{m}$ to the higher integer), the IQ and the FR regime are considered reached whenever all agents have at most $\varepsilon \times m$ instances of $\mathrm{M}$ and, moreover, in the case of the IQ state a group of agents have $(1-\varepsilon) \times m$ instances of $\mathrm{L}$ and the rest have $(1-\varepsilon) \times m$ of $\mathrm{H}$, and in the case of FR all agents have a combination of $(1-\varepsilon) \times m$ instances of both $\mathrm{L}$ and $\mathrm{H}$. In short, a simulation run stops when either it satisfies one of the $\mathrm{C} 1$ stop conditions or it reaches the final time period $\mathrm{T}_{\mathrm{f}}$.

Figure 4 plots the frequency distribution of the stop conditions reached by all simulations we run. As one may expect, the system reaches one of the persistent regimes previously defined in the majority of the cases $(80.98 \%$ of the runs). A relevant result is that

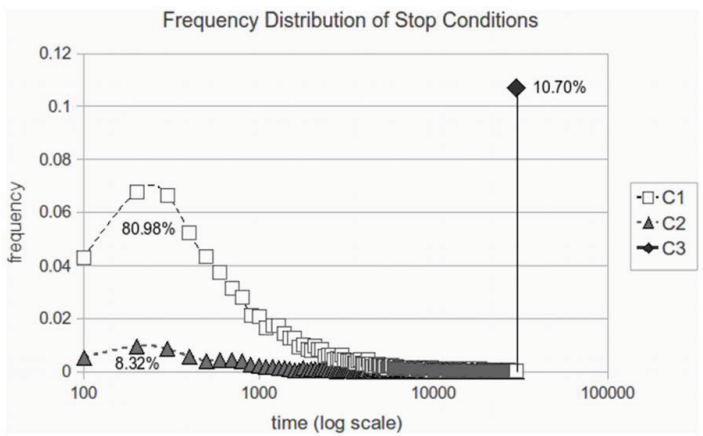

Figure 4. Frequency distribution of the stop conditions reached by $\mathbf{1 0 . 0 0 0}$ simulation runs. $\mathrm{C} 1$ represents the relative frequency of runs that reached one of the $C 1$ stop conditions defined in section 'Most Frequent Persistent Regimes'. C2 represents the frequency of runs that reached the C2 stop conditions defined in section 'Isolated Bargaining Clusters'. This stop criterion extends the C1 conditions to disconnected interaction components that can randomly appear in the spatial distribution of agents on the grid. Finally C 3 gathers the rest of the runs, which are analysed in section 'Other Persistent Regimes'.

doi:10.1371/journal.pone.0017661.g004

(j). PLoS ONE I www.plosone.org 
Mesoscopic Effects: ABM Model in Regular Lattices

even when the assumption of regular spatial structure of interaction, all the persistent regimes obtained in the global interaction case are also reached. Figure 5 illustrates graphically the most frequent states and regimes through a set of simplexes of some representative runs. The regime is characterized by the corresponding pair of simplexes of both intertype and intratype bargaining. Some of these states can be interpreted from a socia perspective as a divided underclass oppressed by a unified elite, as class distinctions, discriminatory regimes, etc. (see [1] for a deeper insight on some interpretations).

Isolated Bargaining Clusters

Although the analysis of the simulation results described in the previous section explains more than 80 percent, it still leaves out a significant set of them. A preliminary visual exploration of some anomalous cases gives us a possible answer: the presence of disconnected groups of agents which play the bargaining game isolated from other groups. In the initialization of the model, agents are randomly distributed and consequently most of spatial distribution samples have agents of both tags dispersed in the lattice, but close enough to make the dynamics interdependent. However, sometimes this randomness produces the formation of two or more isolated groups, i.e. groups of agents who decide their (intertype or intratype) demands without any direct or indirect influence from the agents that belong to other groups. This possibility had not been considered when we defined the C1 stop conditions, so when this event happens the simulation may reach the final time period if groups evolve to different regimes. It is important to notice that the intratype and intertype interaction networks are formed in the random initialization process and are fixed until a stop criterion is reached. Other relevant research in coevolving games does not assume fixed interaction networks but instead the structure dynamically emerges as a consequence of the game. Some of these coevolutionary rules have been used to model

Intertype simplex

Intratype simplex
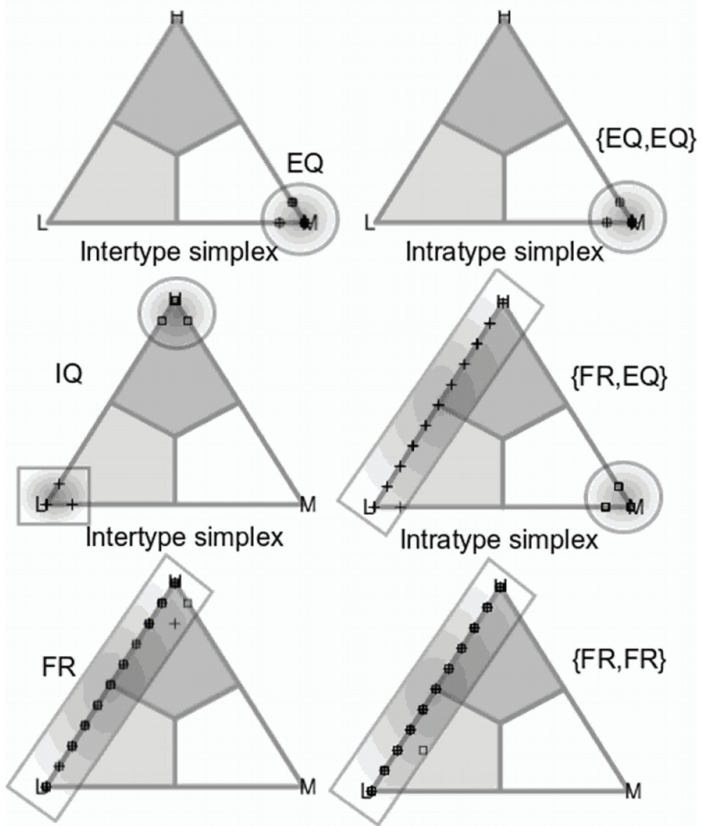

Figure 5. Most frequent persistent regimes of the transient dynamics for the intertype and intratype bargaining games. The bargaining between different groups (intertype) can reach the EQ state (top-left simplex), the IQ state (middle-left simplex) or the FR regime (bottomleft simplex). In the bargaining within groups (intratype) we have shown the combination of $\{\mathrm{EQ}, \mathrm{EQ}\}$ when both groups coordinate in the $E Q$ state (top-right simplex), $\{\mathrm{EQ}, \mathrm{FR}\}$ when one group is in the EQ state but the other is in the FR regime (middle-right simplex), and when both groups stay in the $F R$ regime (bottom-right simplex). doi:10.1371/journal.pone.0017661.g005

(j). PLoS ONE I www.plosone.org 
mechanisms of learning [41], conditional dissociation [42], unilateral and mutual choice in group dynamics [43,44], reputation-based partner choice [45] or the formation and deletion of strategy-independent links [46-48].

We illustrate these cases with one of the runs that exhibits this type of spatial distribution (see Figure 6). In particular, the example run has two disconnected white-tagged groups that reach different final regimes. In order to discriminate this sort of cases we define the $C 2$ stop conditions which are exactly the same conditions as $\mathrm{C} 1$ but applied at the level of disconnected groups -or
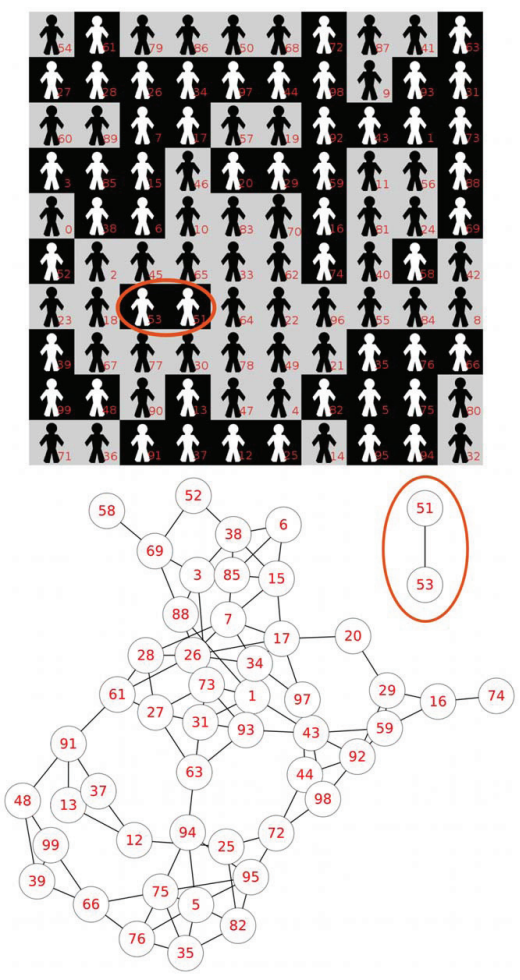

Figure 6. An example case that shows two disconnected groups in the white tag intratype interaction network. Upper figure: spatial distribution of agents with two disconnected groups within white-tagged agents. Lower figure: the corresponding white tag intratype interaction network in which the partition of the network is easily seen (the 51-53 couple vs the rest of white agents). Although it has not been mentioned, note that it is not difficult to identify two other disconnected groups within black agents in this example (the 6089 couple vs the rest of the black agents).

doi:10.1371/journal.pone.0017661.g006

(4). PLOS ONE I www.plosone.org components, in the terminology of network theory that we will use in the next section-, instead of at the level of the whole population, as we do to define $\mathrm{C} 1$ conditions.

Introducing the $\mathrm{C} 2$ stop conditions, the number of runs that end in some of the persistent regimes described so far increases until it reaches more than 89 percent of them (see Figure 4). The remaining set of runs, which end without reaching any of the expected regimes, i.e. C3 stop conditions, are analysed in detail in the next section.

Introducing the $\mathrm{C} 2$ stop conditions, the number of runs that end in some of the persistent regimes described so far increases until it reaches more than 89 percent of them (see Figure 4). The remaining set of runs, which end without reaching any of the expected regimes, i.e. C3 stop conditions, are analysed in detail in the next section.

\section{Other Persistent Regimes}

The results above do not capture all persistent regimes in the game. As evidence of intensive simulation in the spatial game with random configurations, we find that there are still situations that need a much longer time to stop with one of the two criteria $(\mathrm{Cl}$ and C2). This fact could suggest the appearance of some other basins of attraction beyond the original AEY regimes that we have found in section 'Most Frequent Persistent Regimes' and the mentioned combinations of isolated states of section 'Isolated Bargaining Clusters'. This implies that there are additional situations where the transient dynamics of the system differs from the long-run behaviour of the system.

A visual inspection of the tag spatial distribution of these cases puts forward some effects of the topology of interaction that could explain additional regimes. This happens when there are connected clusters of agents with the same tag who play different types of intratype coordination in each of the clusters. The key difference with the cases analysed in the previous section is that such clusters are indeed connected.

It seems clear that the structure of interaction has an influence on the game dynamics. We can consider the structure of intratype interaction as an undirected network where each player represents a node and there is a link between two nodes if both players can play the intratype game (i.e. they are spatial neighbours and they have the same tag). Our hypothesis is that the behaviour of the system is affected by the topological properties in the mesoscale, between the individual and the whole population, of this underlying interaction network.

One of the most relevant mesoscopic characteristics in a network is the property of community structure. Informally, a community in a network consists of a subset of nodes that are relatively densely connected to each other but sparsely connected to other dense groups [49]. This type of local structure can be easily identified in a variety of social contexts: families, friendship circles, virtual groups in the Internet, neighbourhoods, etc. In fact, there is a very rich and growing literature of networks that present community structure, going from the networks of committee and subcommittee assignments in the United States House of Representatives [50], scientific collaboration networks [51], to networks of e-mail interactions between university employees [52] or the collaboration network of jazz musicians [53]. We presume that in connected networks that present strong community structure, different communities can reach different persistent regimes, and the spread of one of the regimes to the whole connected group can be obstructed if the inter-community connectivity is low.

We illustrate the intuition of this phenomenon in the following idealized case. In Figure 7 we represent a certain configuration of tags and the underlying intratype interaction network of white- 
Mesoscopic Effects: ABM Model in Regular Lattices

(48)

(35)

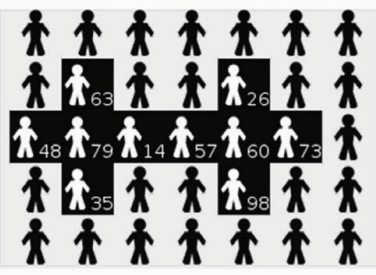

(79)

(63) (14)

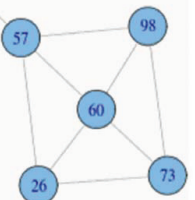

Figure 7. Idealized case of white-tagged players and the underlying intratype interaction network. We have analysed the effects that appear in the stylized configuration showed on the left of the figure. On the right, we represent the underlying interaction structure for the intratype

game of white-tagged players.
doi:10.1371/journal.pone.0017661.g007

tagged players. Intuitively there are two communities in the network (depending on the algorithm used to identify communities, there may be other partitions in communities different to the presented in the example). If we play this game repeatedly a frequent result is showed in Figure 8. Each community reaches a different regime, stays trapped in it for a long time, and the diffusion of a general homogeneous behaviour in the game is hindered.

If we want to extend these results to more general conditions in the lattice game, we need to specify exactly how to define the concept of community beyond the intuitive and vague idea of

some nodes very connected among them and sparsely linked with other communities. As a matter of fact, the problem of detecting communities is very challenging for two reasons: first, the number of possible partitions is huge for non-trivial networks, and second, but no less important, the concept (and hence the preferred definition of communities) may be domain-specific, depending on the field of application. Given this, it is not surprising that nowadays there is a wide plethora of methods based on different techniques and ideas to define and to identify communities in networks (see some recent reviews in [49,54-57]).
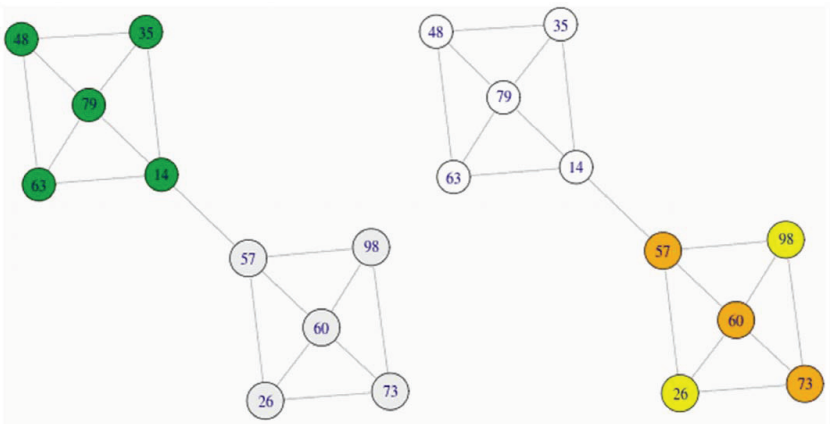

Best reply: $\mathrm{M}$ (white),L(yellow), $\mathrm{H}$ (orange)

Figure 8. Partition in communities of the idealized case and final regimes. On the right, we can observe two different persistent regimes in the intratype game of white-tagged players depicted in Figure 7 . The different regimes correspond exactly to the different communities showed on

the left.
doi:10.1371/journal.pone.0017661.g008

(2). PLOS ONE I www.plosone.org 
In our analysis of the spread or lock-in of the persistent regimes, the idealized case gives us a hint to select the identifying community algorithm. We see that the edges that separate communities act as bottlenecks that enable or put obstacles to the flow of strategies. Based on this idea, Girvan and Newman [51] defined the concept of betweenness of an edge generalizing the concept of betweenness of a node by Freeman [58]. The betweenness of an edge is calculated as the number of geodesic (i.e. shortest) paths between node pairs that run through it, normalized dividing by the number of pairs of nodes. The betweenness of an edge gives us an idea of the importance of the link to stop the flow of information in the network.

The algorithm of Girvan and Newman requires calculating the betweenness of all edges in the network and removing the one with the highest betweenness, repeating the whole process until no edges remain (in case of tie, one can be randomly removed, or al can be simultaneously removed). The logic of the algorithm is based on the idea that the edges connecting communities will have comparatively high betweenness and hence, by removing them iteratively, we will separate the different components of the network that reveal the hidden community structure of the graph The result of this algorithm is a dendrogram where horizontal cross-sections represent different possible community divisions, depending on the desired number of communities. Since the method does not provide the appropriate number of communities to split the network, the same authors [59] proposed to evaluate the divisions using the concept of modularity as the fitnes function. The modularity of a partition is an index that aims to quantify how good a partition is. Partitions with high values of modularity are those in which there are dense internal connections between the nodes within clusters but only sparse connection between different clusters. Modularity compares the number of links inside a community with the expected number of links that one would find in the community if the network were randomly generated keeping the degree of every node (i.e. the number of links), but linking them randomly. Following Newman [60], the modularity $Q$ of an unweighted and undirected network partitioned into communities can be computed as:

$$
Q=\sum_{i}\left(e_{i i}-b_{i}^{2}\right)
$$

where $e_{i i}$ denotes the fraction of all edges that have both ends in community $i$, and $b_{i}$ is the fraction of edges that have one or two ends in community $i$.

Given that this algorithm to partition the network formalizes the idea of information flow, we hypothesize that some additional persistent regimes can appear when each community adopts a coordinated regime except for potentially some border agents with other communities that can present a fluctuating behaviour depending on the community with which they play, and hence act as bottlenecks for the diffusion of norms between communities.

In order to check our hypothesis we have analysed the instances where simulations have not reached any of the persistent regimes considered in the previous sections: the simulations that stopped because they reached the final time period $\mathrm{T}_{\mathrm{f}}$, i.e. stop condition C3. In each one of these cases we have recorded the final state of each player in the intratype game according to the definitions of section 'Isolated Bargaining Clusters'. If the agent did not reach any of the predefined states based on her memory, we classify her as "regime not established".

In those games, we have also exported the topology of each component of the underlying intratype interaction network and applied the Girvan-Newman algorithm maximizing the modularity to identify the different communities. We can compare the partition given by the algorithm with the final behavioural state of the players in the game. If the mesoscopic topology conditions the spread and diffusion of strategies in the lattice, the state of the players should be homogeneous in each community except for potentially some nodes that are at the border of the community. We define a node as border in a community if she has a link to another player that belongs to a different community. When two connected communities stabilize in a different regime, the agents that are at the border should present a flipping strategy, as a consequence of their exposure to different regimes. In fact, given the construction of the Girvan-Newman algorithm, the interaction of an agent that is at the border of a community with the neighbour community is done by means of links of high betweenness. In general, the frequency of interaction of those agents with players in the neighbour community is going to be lower than with players in their own community. In terms of diffusion of regimes this fact is crucial, since in order to change their strategy they would need to play very often with players from the other communities, which is against the chances imposed by the topology. Agents at the border act as buffers and stabilizers of the diffusion of regimes.

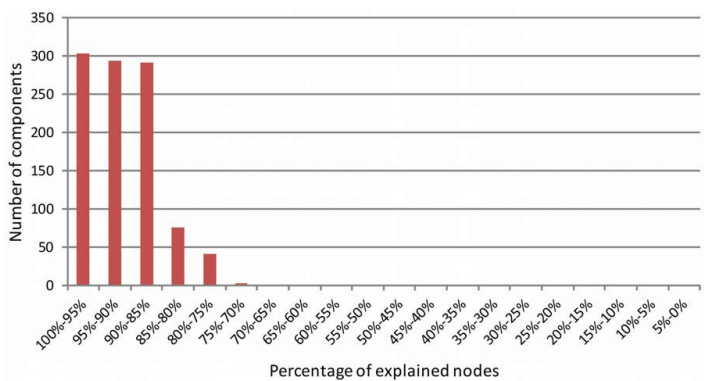

Figure 9. Number of components analysed that have a given percentage of explained nodes (i.e. nodes that have a homogeneous strategy with their communities, or border nodes with a different strategy to that in their community). doi:10.1371/journal.pone.0017661.g009 


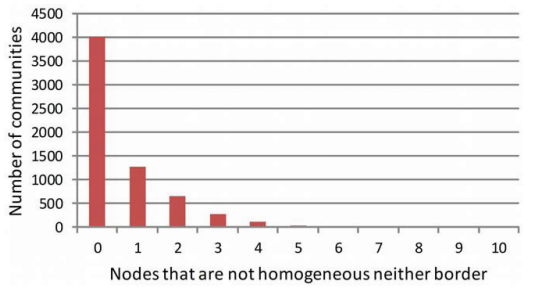

Figure 10. Number of communities identified by the GirvanNewman algorithm which have none or more nodes that are not homogeneous neither border.

doi:10.1371/journal.pone.0017661.g010

In the 1007 components from simulations that finished with the C3 criterion, we have computed the number of nodes that have a homogeneous strategy with the community where they belong, and the number of border players that have a different behaviour. The number of nodes in this category accounts for $91.7 \%$. We represent in Figure 9 the percentage of nodes explained in the final state of the 1007 components.

Our community analysis of the 1007 networks has identified 6,366 communities. We have also computed the number of players within a community proposed by the algorithm that have a discordant behaviour according to our hypothesis. Results are presented in Figure 10. As we can see more than $60 \%$ of the communities have exactly the expected behaviour.

The $8 \%$ of nodes that present a strategy discordant with the expected behaviour can be explained by different reasons. First of all, it is important to notice that the model is stochastic, and hence some randomness is going to be present. This randomness may introduce important inertia in the analysis of the state of the players. Another factor could be that we are stopping the simulations after 30000 ticks, which for some complex topologies may be insufficient to converge to a complete persistent regime. Apart from that, we should keep in mind that the topologies analysed are obtained from complete random initialization of agents in the lattice which may produce strange topologies. The partition in communities of such topologies can be different depending on the algorithm used. The Girvan-Newman algorithm is very appealing to explain diffusion processes because it is based on a centrality measure but other algorithms are better at maximizing the modularity [54]. It is possible, therefore, that other algorithms give us other partition that improve the explanation based on the Girvan-Newman algorithm. In any case, this study has shown the significant effect of the mesoscopic interaction structure in the spatial diffusion of strategies of the game in the lattice.

\section{References}

1. Axtell RL, Epstein JM, Young HP (2001) The Emergence of Classes in a Multi-Agent Bargaining Model. In: Durlauf SN, Young HP, eds. Social Dyna

2. Coleman JS (1990) Foundations of social theory. CambridgeMassachusetts: Belknap Press of Harvard University Press.

Kandori M (1992) Social Norms and Community Enforcement. Review of Economic Studies 59(1): 63-80.

4. Nash JF (1950) The Bargaining Problem. Econometrica 18(2): 155-162.

PLOS ONE | www.plosone.org

\section{Discussion}

In this work we have addressed the effect of a regular spatial structure on the Nash bargaining game in a finite population of tagged agents. We have showed that all the transient regimes proposed by Axtell et al [1] can also be present in the lattice game. More interestingly, depending on the particular tag distribution of agents through the grid we have found some topological properties that explain the diffusion of the agent's strategies in the lattice. We have showed that isolated clusters of intra or inter type of interaction can reach different persistent regimes. Moreover, we have proved the influence of the topology in understanding new stable regimes different from those found by Axtell et al [1]. To explain their appearance and persistence, we have based our analysis on the mesoscopic properties of the interaction structure, concretely in the community structure of the network of interaction. Using the Girvan-Newman algorithm based on the edge betweenness and the concept of modularity to identify communities, we can understand the behaviour of many of the nodes of the simulation that do not reach any of the previous described regimes. Although the results can be dependent on the rules of the game, they may explain the emergence of different norms of economic interaction and resource allocation among different spatial groups, not only if the groups are isolated and do not communicate among them, but also if the interaction among groups has community structure.

The findings of these mesoscopic effects in a property distribution game strongly corroborate the relevance of the arguments previously exposed by authors like Lozano et al. $[24,25]$ in the evolutionary Prisoner's Dilemma, Roca et al. [61] and Tomassini and Pestelacci [62] in cooperation dilemmas, and similar phenomena also described by Castelló et al. [63,64] in the context of dynamical models of competing options. Although the game played on the spatial substrate is different from the games explored by these authors, the mechanism that prevents the homogenization of a general strategy in the population is very similar. They use the idea of topological traps $[61,63,64]$ (i.e. links between nodes of different degrees in regions with few or no redundant paths) to explain why "homogeneous strategy waves" do not propagate over the network uniformly. Since we are partitioning the network using a methodology based on the concept of betweenness, we are indirectly detecting the notion of topological traps in the borders between communities, as our results show.

\section{Acknowledgments}

We would like to thank Luis R. Izquierdo, Segismundo Izquierdo, Cesáreo Hernández, Javier Pajares and two anonymous reviewers for some advice and comments on this manuscript. We would also like to thank the participants in the SiCoSSys Project workshop held in Valladolid in December 2010.

\section{Author Contributions}

Conceived and designed the experiments: DJP JIS JMG ALP. Performed the experiments: DJP JIS JMG ALP. Analyzed the data: DJP JIS JMG ALP. Contributed reagents/materials/analysis tools: DJP JIS JMG ALP. Wrote the paper: DJP JIS JMG ALP.
. Skyrms B (1996) Evolution of the social contract. Cambridge: Cambridge University Press

We, Cuesta JA, Sánchez A (2009) Evolutionary game theory: Temporal 208-249. doi: 10.1016/j.plrev.2009.08.001.

Young HP (1998) Individual strategy and social structure, An evolutionary theory of institutions. Princeton, N.J: Princeton University Press.

8. Young HP (1993) An Evolutionary Model of Bargaining. Journal of Economic Theory 59(1): 145-168. doi: 10.1006/jeth. 1993.1009. 
9. Alexander J, Skyrms B (1999) Bargaining with Neighbors: Is Justice Contagious? The Journal of Philosophy 96(11): 588-598. doi: 10.2307/2564625.

10. Charness G, Corominas-Bosch M, Frechette GR (2007) Bargaining and network structure: An experiment. Journal of Economic Theory 136(1): 28-65. do: $10.1016 /$ j.jet.2005.06.009

11. Ohtsuki H, Pacheco JM, Nowak MA (2007) Evolutionary graph theory Breaking the symmetry between interaction and replacement. Journal of Theoretical Biology 246(4): 681-694. doi: 10.1016/j.jtbi.2007.01.02

12. Ohtsuki H, Nowak MA, Pacheco JM (2007) Breaking the symmetry between interaction and replacement in evolutionary dynamics on graphs. Physic

13. Fort H, Pérez N (2005) The Fate of Spatial Dilemmas with Different Fuzzy Measures of Success. Journal of Artificial Societies and Social Simulation 8(3, http://jasss soc surrey ac uk $/ 8 / 3 / 1 \mathrm{html}$

14. Nowak MA, May RM (1992) Evolutionary Games and Spatial Chaos. Nature 359(6398): 826-829. doi: 10.1038/359826a0.

15. Perc M, Wang Z (2010) Heterogeneous Aspirations Promote Cooperation in the Prisoner's Dilemma Game. PLoS ONE 5(12): e15117. doi: 10.1371/journal. pone. 0015117

16. Gómez-Gardeñes J, Campillo M, Floría LM, Moreno Y (2007) Dynamical organization of cooperation in complex topologies. Physical Review Lette 98(10). doi: 10.1103/PhysRevLett.98.108103.

17. Poncela J, Gómez-Gardeñes J, Floría LM, Moreno Y (2007) Robustness of cooperation in the evolutionary prisoner's dilemma on complex networks. New

18. Fu F, Liu LH, Wang L (2007) Evolutionary Prisoner's Dilemma on heterogeneous Newman-Watts small-world network. European Physica Journal B 56(4):

19. Perc M (2009) Evolution of cooperation on scale-free networks subject to error 0. Santos FC, Pacheco JM (2005) Scalc-Free Networks Provide a Unifying Framework for the Emergence of Cooperation. Physical Review Letters $95(9$ 098104. doi: 10.1103/PhysRevLett.95.098104.

21. Santos FC, Pacheco JM, Lenaerts T (2006) Evolutionary dynamics of social dilemmas in structured heterogeneous populations. Proceedings of the Nationa Academy of Sciences of the United States of America 103(9): 3490-3494. do

22. Szolnoki A, Perc M, Danku Z (2008) Towards effective payoffs in the prisoner's dilemma game on scale-free networks. Physica A: Statistical Mechanics and

23. Poncela J, Gómez-Gardeñes J, Floría LM, Moreno Y, Sánchez A (2009) Cooperative scale-free networks despite the presence of defector hubs. Europhysics Letters 88(3): 38003. doi: 10.1209/0295-5075/88/38003.

24. Lozano S, Arenas A, Sánchez A (2008) Community connectivity and heterogeneity: Clues and insights on cooperation on social networks. Journal
of Economic Interaction and Coordination 3(2): 183-199. doi: 10.1007/s1 1403008-0041-7.

25. Lozano S, Arenas A, Sánchez A (2008) Mesoscopic structure conditions the emergence of cooperation on social networks. PLoS ONE 3(4): el892. do: 10.1371/journal.pone. 0001892

26. Hauert C, Doebeli M (2004) Spatial structure often inhibits the evolution of cooperation in the snowdrift game. Nature 428(6983): 643-646. doi: $10.1038 /$

. Sysi-Aho M, Saramäki J, Kertész J, Kaski K (2005) Spatial snowdrift game with myopic agents. European Physical Journal B 44(1): 129-135. doi: 10.1140/epjb/

28. Tomassini M, Luthi L, Giacobini M (2006) Hawks and Doves on small-world networks. Physical Review E - Statistical, Nonlinear, and Soft Matter Physics 73(1). doi: 10.1103/PhysRevE.73.016132

29. Han-Xin Y, Kun G, Xiao-Pu H, Bing-Hong W (2008) Evolutionary snowdrif game on heterogeneous Newman Watts small-world network. Chinese Physics 17(8): 2759. doi: $10.1088 / 1674-1056 / 17 / 8 / 002$

30. Helbing D, Szolnoki A, Perc M, Szabo G (2010) Punish, but not too hard: How costly punishment spreads in the spatial public goods game. New Journal of Physic

31. Helbing D, Szolnoki A, Perc M, Szabo G (2010) Evolutionary establishment of moral and double moral standards through spatial interaction

32. Helbing D, Szolnoki A, Perc M, Szabo G (2010) Defector-accelerated (10. Physic Review E 81(5): 057104. doi: 10.1103/PhysRevE.81.057104.

33. Szolnoki A Perc M (2010) Reward and cooperation in the spatial public good Szolnoki A, Perc M (2010) Reward and cooperation in the spatial public good
game. Europhysics Letters 92(3): 38003. doi: 10.1209/0295-5075/92/38003. 4. Chen J. Quan H (2009) Effect of imitation in evolutionary minority game on small-world networks. Physica A: Statistical Mechanics and its Application 388(6): 945-952. doi: 10.1016/j.physa.2008.11.03.

35. Kirley M (2006) Evolutionary minority games with small-world interaction Physica A: Statistical Mechanics and its Applications 365(2): 521-528. do: 10.1016/. jphysa.2005.10.002 6. Szabo G, Fath G (2007) Evolutionary games on graphs. Physics Reports $446(4$
6): 97-216. doi: $10.1016 /$ j.physrep.2007.04.004.
37. Perc M, Szolnoki A (2010) Coevolutionary games-A mini review. BioSystems 99(2): 109-125. doi: 10.1016/j.biosystems.2009.10.003

38. Poza D, Villafañez F, Pajares J, Lopez-Paredes A, Hernandez C (2011) New insights on the Emergence of Classes Model. Discrete Dynamics in Nature and Society. DDNS/915279. Volume 2011. doi: 10.1155/2011/915279.

39. Izquierdo LR, Izquierdo SS, Galán JM, Santos JI (2009) Techniques to understand computer simulations: Markov chain analysis. Journal of Artificial Societies and Social Simulation 12(1): 6. http://jasss.soc.surrey.ac.uk/12/1/6. html.

40. Floría LM, Gracia-Lázaro C, Gómez-Gardeñes J, Moreno Y (2009) Social network reciprocity as a phase transition in evolutionary cooperation. Physical PhysRevE.79.026106.

41. Skyrms B, Pemantle R (2000) A dynamic model of social network formation. Proceedings of the National Academy of Sciences of the United States of America 97(16): 9340-9346. doi: 10.1073/pnas.97.16.9340.

42. Izquierdo SS, Izquierdo LR, Vega-Redondo F (2010) The option to leave Conditional dissociation in the evolution of cooperation. Journal of Theoretical Biology 267(1): 76-84. doi: 10.1016/j.jtbi.2010.07.039

43. Yamashita T, Izumi K, Kurumatani K (2005) An investigation into the use of group dynamics for solving social dilemmas. Lecture Notes in Artificial

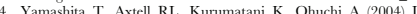

(204) Investigation of mutual choice metanorm in group dynamics for solving social dilemmas. Lecture
Notes in Artificial Intelligence 3012: 137-153. doi: 10.1007/978-3-540-246664_9.

45. Fu F, Hauert C, Nowak MA, Wang L (2008) Reputation-based partner choice promotes cooperation in social networks. Physical Review E - Statistical, Nonlinear, and Soft Matter Physics 78(2). doi: 10.1103/PhysRevE.78.026117. 6. Szolnoki A, Perc M (2009) Resolving social dilemmas on evolving random networks. Europhysics Letters 86(3). doi: 10.1209/0295-5075/86/30007.

47. Szolnoki A, Perc M (2009) Emergence of multilevel selection in the prisoner's dilemma game on coevolving random networks. New Journal of Physics 11: doi

48. Wu B, Zhou D, Fu F, Luo Q Wang L, Traulsen A (2010) Evolution of cooperation on stochastic dynamical networks. PLoS ONE 5(6): ell1187. doi: cooperation on stochastic dyna
10.1371 journal.pone.0011187.

49. Porter MA, Onnela JP, Mucha PJ (2009) Communities in networks. Notices of the American Mathematical Society 56(9): 1082-1097. doi: 10.1063/1.3194108.

50. Porter MA, Mucha PJ, Newman MEJ, Friend AJ (2007) Community structure in the United States House of Representatives. Mhysica A: Statistical Mechanic and its Applications 386(1): 414 438. doi: 10.1016/j.physa.2007.07.039.

51. Girvan M, Newman MEJ (2002) Community structure in social and biological networks. Proceedings of the National Academy of Sciences of the United States

52. Guimera R, Danon L, Diaz-Guilera A, Giralt F, Arenas A (2006) The real
of Americat communication network behind the formal chart: Community structure in rranizations Journal of Economic Behavior and Organization 61/4): 653-667. doi: 10.1016/j.jebo.2004.07.021

53. Gleiser P, Danon L (2003) Community Structure in Jazz. Advances in Complex Systems 6(4): 565-573. doi: 10.1142/S0219525903001067.

54. Danon L, Diaz-Guilera A, Duch J, Arenas A (2005) Comparing community structure identification. Journal of Statistical Mechanics: Theory and Experiment;9): 219-228. doi: 10.1088/1742-5468/2005/09/P09008

55. Fortunato $\mathrm{S}$ (2010) Community detection in graphs. Physics Reports 486(3-5): 75-174. doi: 10.1016/j.physrep.2009.11.002.

. Detecting community structure in networks. European Physical Journal B 38(2): 321-330. doi: 10.1140/epjb/e2004-00124-y

. Lancichinetti A, Saramäku J, Kivelà M, Fortunato S (2010) Characterizing the community structure of comp 10.1371 journal.pone.0011976.

58. Freeman LC (1977) A set of measures of centrality based upon betweenness Sociometry $40: 35-41$

59. Newman MEJ, Girvan M (2004) Finding and evaluating community structure in networks. Physical Review E - Statistical, Nonlinear, and Soft Matter Physics 69(22). doi: 10.1103/PhysRevE.69.026113.

60. Newman MEJ (2004) Fast algorithm for detecting community structure in networks. Physical Review E - Statistical, Nonlinear, and Soft Matter Physics

61. Roca CP, Lozano S, Arenas A, Sánchez A (2010) Topological traps control flow on real networks: The case of coordination failures. PLoS ONE 5(12): el5210. Toi: $10.1371 /$ journal pone 0015210

Testelacci E (2010) Evolution of coordination in social networks: A numerical study. International Journa
doi: $10.1142 / \mathrm{S} 012918311001583 \mathrm{X}$

63. Castelló X, Toivonen R, Eguíluz VM, Saramäki J, Kaski K (2007) Anomalou lifetime distributions and topological traps in ordering dynamics. Europhysics Letters 79: 66006. doi. 10.1209/0255-5075/79/6600.

64. Toivonen R, Castelló X, Eguiluz VM, Saramäki J, Kaski K, San Miguel M 2009) Broad lifetime distributions for ordering dynamics in complex networks Physical Review E - Statistical, Nonlinear, and Soft Matter Physics 79(1). doi: 

Evolution of the Equity Norm in Small World Networks 



\title{
Evolution of Equity Norms in Small-World Networks
}

\author{
Authors and Affiliations \\ José I. Santos ${ }^{1}$, David J. Poza ${ }^{2}$, José M. Galán ${ }^{1}$ and Adolfo López-Paredes ${ }^{2}$ \\ ${ }^{1}$ Grupo INSISOC. Área de Organización de Empresas. Dpto. de Ingeniería Civil. Escuela \\ Politécnica Superior. Universidad de Burgos. Edificio La Milanera, C/ Villadiego S/N, 09001 \\ Burgos. Spain. jisantos@ubu.es; jmgalan@ubu.es \\ 2 Grupo INSISOC. Dpto. de Organización de Empresas y CIM. Escuela de Ingenierías \\ Industriales. Universidad de Valladolid. Paseo del Cauce 59, 47011 Valladolid. Spain. \\ djpoza@gmail.com; adolfo@insisoc.org
}

\section{Abstract}

The topology of interactions has been proved very influential in the results of models based on learning and evolutionary game theory. This paper is aimed at investigating the effect of structures ranging from regular ring lattices to random networks, including small-world networks, in a model focused on property distribution norms. The model considers a fixed and finite population of agents who play the Nash bargaining game repeatedly. Our results show that regular networks promote the emergence of the equity norm, while less structured networks make possible the appearance of fractious regimes. Additionally, our analysis reveals that the speed of adoption can also be affected by the network structure.
\end{abstract}

\section{Introduction}

The emergence, prevalence and collapse of social norms in groups have attracted scientists from a wide range of disciplines [1-5]. Social norms are appealing because they can act as mechanisms for regulating individual behaviour without the need of a central authority. Social norms are indeed rules that are socially enforced $[6,7]$.

In the field of Economics, there are plenty of transactions that are commonly regulated by means of habits, repeated interaction, community enforcement, social pressure, trust or reputation, rather than by formal contracts [8]. In particular, among the set of economic interactions that are often modulated by social norms, we can find those that govern the distribution of property within a group. In this context, social norms sometimes favour equitable distributions, but this is not always the case. Social norms can also contribute to the persistence of discriminatory allocations, often supported by observable differences in individual characteristics or group membership, such as gender, race, ethnicity, age, caste, etc.

Learning game theory provides a useful framework to analyse this type of norms formally [912]. Social interactions are modelled as games played by actors that use the history of the game to form expectations or beliefs about the other players' behaviour, and consequently 
select an appropriate strategy. In general, not all conceivable groups of players within a population will be equally likely to interact, i.e. the population may be somewhat structured. In such cases, networks are particularly useful to describe the (sub)set of interactions that may take place: a player can only directly interact in the game with his neighbours in the network.

Relaxing the assumption of global interaction and using sophisticated learning rules usually reduces the analytic tractability of the models, and accentuates the relative usefulness of computer simulation for exploration and analysis. Given the explicit correspondence between players in the model and computational entities in the simulation, those players are naturally implemented as agents in an agent-based model $[13,14]$. This approach is increasingly used in social and economic models [15-20].

Concretely, in the case of property distribution norms, interactions are often modelled as Nash bargaining games (also known as Nash demand games) [21]. This game consists of two players that have to divide a sum of money among them. Each player demands a share without knowing the demand of the other. If the sum of their individual demands does not exceed the total, the payoff for each player is the amount of money they asked for; however, if the sum of the two demands exceeds the total, they both obtain nothing. Based on this game and its posterior evolutionary version [22], Axtell, Epstein and Young [23] designed an agent-based model (henceforth AEY's model) to understand the transient and the asymptotic dynamics of the Nash bargaining game in a finite population. In their model, they assumed that the players can make three possible demands only: low (L), medium (M) and high $(\mathrm{H})$ and agents play a noisy best reply to their past experience. The model shows that several persistent regimes different from the equity norm can appear and perpetuate under several learning rules and combination of parameters $[23,24]$.

AEY's model has been extended to understand the effect of spatial structure. In particular it has been analysed in regular square lattices with a fixed finite population of tagged agents [25]. This study revealed that that the mesoscopic properties of the interaction networks have a significant impact on the diffusion of strategies. However, real networks usually differ from the regular lattice topology [26]. To get deeper insights on the effect of social structure in the diffusion of norms, we analyse AEY's model in networks that may present the so-called smallworld effect [27], i.e. networks where the average distance between agents is relatively short.

The scientific origin of small-world research is attributed to the pioneering work of Pool and Kochen [28] and Milgram [29]. Nevertheless, the puzzle of how to explain the evidence that several real networks are highly clustered (as lattices for example), and at the same time show the small-world effect (like e.g. random networks), was not envisioned until the seminal work of Watts and Strogatz [30]. In their work they proved that both properties of real networks could be embodied in a simple mathematical network algorithm that interpolates between order and randomness. In the transition, they found a class of networks -small-world networks- displaying high clustering and the small-world effect simultaneously.

Models of dynamical systems embedded in small-world networks display different global behaviour due to enhanced signal-propagation speed, computational power, and synchronizability $[30,31]$. The effect of this type of topology has been investigated by the academic community, examples of which include the analysis of iterated games such as Hawk- 
Dove [32,33], Prisoner's Dilemma [34,35], Minority Game [36] or Ultimatum Game [37] but also in diffusion models [38].

In this article we have extended the analysis of dynamic norm diffusion in a population considering AEY's model as a framework. We have analysed the influence of the small-world topology on the results of the game. To this aim, we have organized the paper as follows: first, we briefly explain the extensions and modifications that we have performed on AEY's original model and the main properties of the network generator mechanism based on the WattsStrogatz algorithm [30]. Next, we analyse the Markovian properties of the unperturbed and perturbed model. Subsequently we characterize the equity norm from an agent's perspective and define the concepts and mechanisms used to analyse the dynamics of the model. In the results section we design and discuss a set of experiments to analyse the frequency of states, the diffusion of the equity norm and the effect of the size of the population. We then finish with the conclusions of this work.

\section{The Model}

The model proposed in this article is based on AEY's model [23]. In their abstraction, agents are randomly paired up to play a Nash Demand Game [21]. Agents play a game in which each of them can demand three possible portions of a virtual cake (which is a metaphor for a piece of available property): a low ( $\mathrm{L} ; 30 \%)$, a medium ( $\mathrm{M} ; 50 \%)$ or a high $(\mathrm{H} ; 70 \%)$ share. Agents get what they demand as long as the sum of the two demands is no more than one hundred per cent of the pie. Otherwise, they get nothing (see the payoff matrix used in the model in Table 1).

Agents are endowed with a memory (of size $\mathrm{m}$ ) in which they store the portion of the pie demanded by their opponents in the last $m$ rounds. In order to make a decision, in AEY's model, an individual chooses the best reply that maximizes the expected payoff considering their past experiences. In our model we consider a simpler decision rule, which dictates that individuals choose the best reply against the most frequent demand in their memory (ties are resolved randomly without bias). This last rule is cognitively less demanding than AEY's and, naturally, it induces different results than those obtained with the original decision rule [24]. The response is assumed "noisy" in the sense that agents may make mistakes in their decisions (or simply experiment from time to time) with small probability. Hence, with probability $(1-\varepsilon)$ an individual chooses the best reply and with probability $\varepsilon$ she chooses one of the three possible demands at random (low, medium or high with the same probability). Afterwards, agents are paired up again with other agents (chosen at random) and the bargaining process continues.

Table 1. Payoff matrix of the Nash demand game.

\begin{tabular}{c|l|l|l|}
\multicolumn{2}{c}{$H$} & \multicolumn{1}{c}{$M$} & \multicolumn{1}{l}{$L$} \\
\multirow{3}{*}{$H$} & $(0,0)$ & $(0,0)$ & $(\mathbf{7 0 , 3 0 )}$ \\
\cline { 2 - 4 }$M$ & $(0,0)$ & $(\mathbf{5 0 , 5 0 )}$ & $(50,30)$ \\
\cline { 2 - 4 }$L$ & $(30,70)$ & $(30,50)$ & $(30,30)$ \\
\cline { 2 - 4 } & & &
\end{tabular}


The influence of some parameters of the model (such as the number of agents, the memory size, the payoff matrix or the decision rule) has been thoroughly analysed in [24], but that study only considered the situation where every player could interact with any other player (i.e. a complete interaction network).

In a later extension of the model [25], agents were located on a regular square lattice in such a way that one agent could play only with any of her eight surrounding neighbours (Moore neighbourhood). The influence of this topology on the outcome of the Nash Demand Game has been analysed. Results show that the mesoscopic properties of the interaction networks of players with the same tag have an important influence on the diffusion of the emergent norms. The regular square lattice was a first attempt to adapt the AEY's model to more realistic scenarios of human interaction. Nevertheless, regular interaction is still far from most real-life patterns of relation.

The model that we present in this paper is an extension of AEY's model [23] where the agents ${ }^{1}$ are located on a Watts and Strogatz network [30]. Some real human social networks have been proved [26] to be highly clustered (which, roughly speaking, means that your friends' friends are likely to be also your friends) and to have a short average geodesic distance (which means that one can travel from any agent to any other in a small number of hops, the so-called smallworld effect ${ }^{2}$ ). These two properties do not occur simultaneously in regular or in random networks. On the one hand, structured regular networks (such as lattices) are known to be highly clustered but they have long average geodesic distances. On the other hand, random graphs, in which every possible edge occurs independently with a fixed probability, have a short average geodesic distance but they are not highly clustered. Watts and Strogatz [30] designed a mechanism that gives a family of networks which can combine both properties at the same time ${ }^{3}$ : small-world effect and high clustering.

In the model implemented in this article we use the Watts-Strogatz algorithm [30] to create networks with different values of the probability of rewiring $\beta$. This parameter smoothly interpolates between extreme cases of a regular ring lattice and a random network, traversing "small-world" networks along the way (see Figure 1). As the probability of rewiring increases, the network becomes less regular (and thus less clustered) and the appearance of longdistance links reduces the average geodesic distance.

\footnotetext{
${ }^{1}$ In this article the term "agent" -coming from the ABM literature- and the term "node" -coming from the network theory- could be applied indistinctly. However we prefer to use the term "agent" in the description of the agents' behavior and in the interaction network properties.

${ }^{2}$ In small-world networks, average path length scales with the logarithm of the number of agents $\mathrm{N}$ in the network.

${ }^{3}$ In a range of values for the parameter 'probability of rewiring'.
} 


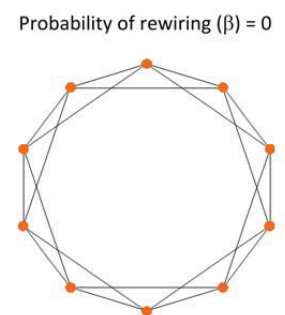

High clustering High average path

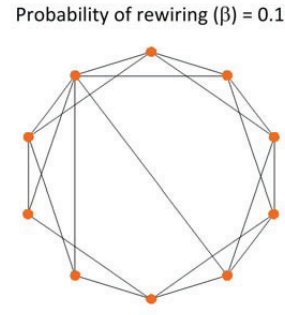

High clustering Low average path

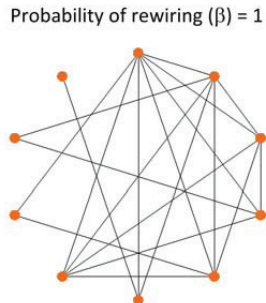

Low clustering Low average path

Figure 1. Network structure for several values of the probability of rewiring. $N$ (number of nodes)=10, $k$ (average degree) $=4$

In our model, the network is created at the beginning of each run and remains fixed thereafter. At each time period, all the agents are selected in a random order to play the Nash Demand Game with one of their (randomly selected) neighbours. It is important to note that each time period consists of $\mathrm{N}$ matches, and consequently it is probable that an agent plays more than once in each period.

In the subsequent experiments we will show how the probability of rewiring (and thus the properties of the resulting network) affects the regimes that can be reached in the AEY's game.

Notice that, unlike in previous works [24,25] and to focus on topological effects, we have not considered the fact that the agents could have tags (i.e. distinguishable labels, such as the colour of the agent, which other agents can identify and condition their decisions on them). In $[23,24]$ the consideration of tags led to two different games (intra and intertype games). In [25], as the agents were placed on a regular grid, each type of game gave rise to a different network (intra and intertype networks). In this work, however, the agents are placed on a small-world network. If we had considered tags in this network, and had split it into two independent networks (as in [25]), the resulting networks wouldn't necessarily have smallworld properties.

\section{General Analysis}

Before doing a computational exploration of the agent-based model, it is particularly interesting to conduct a previous analysis using the framework of Markov Chains [39], to get useful insights about the expected dynamics and behaviour of the model. In terms of Markovian properties, the system is a time-homogeneous Markov Chain. Considering that the interaction network is fixed and known, the state of the system is completely described by a set of $N$ vectors $\left\{X_{i}(t)\right\}^{N}{ }_{i=1}=\left\{L_{i}(t) / m, M_{i}(t) / m, H_{i}(t) / m\right\}^{N}{ }_{i=1}$ of the relative frequencies of opponents' demands for each agent $\mathrm{i}$, where $\mathrm{L}_{\mathrm{i}}(\mathrm{t}), \mathrm{M}_{\mathrm{i}}(\mathrm{t})$ and $\mathrm{H}_{\mathrm{i}}(\mathrm{t})$ denotes the number of times that agent $\mathrm{i}$ 's opponents demanded $\mathrm{L}, \mathrm{M}$ and $\mathrm{H}$ respectively in the $\mathrm{m}$ most recent interactions just before time $t$.

As previously explained, an interaction between two agents is modelled as a Nash demand game [21] with three discrete strategies or decisions $\{H, M, L\}$-the corresponding matrix payoff 
is represented in Table 1. For the one-shot game, there are three pure-strategy Nash equilibriums, one equitable $(M, M)$, and two other (symmetric) inequitable $(H, L)$ and $(L, H)$. These states play a role of focal points that drive the bargaining evolution and explain the asymptotic dynamics of the system.

\subsection{The Unperturbed Model}

The system dynamics are determined by the presence or absence of errors in agents' decisions. In the absence of errors, i.e. the unperturbed model, the system has absorbing states in which sooner or later it will be trapped (if we run the model for long enough). These absorbing states are directly related with the pure-strategy Nash equilibriums just mentioned before. Obviously, the interaction network conditions the probabilities of these states to be reached. Assuming global interactions, i.e. every agent can play with everybody without any restriction, there is only one absorbing state corresponding to the equitable strategy. This happens when everyone in the population expects the others will demand $M$, and consequently everyone demands $\mathrm{M}$, so the system ends up reaching an absorbing state, called equity (EQ) state. This state is equitable because all agents get equal payoffs, and is also efficient (in Pareto sense) because no agent can be made better off without making another agent worse off.

When we assume local interactions, i.e. an agent can only play with her neighbours in the interaction network, besides the EQ state, there are two other absorbing states corresponding to the inequitable strategies ${ }^{4}$. This happens when there are two separated groups of agents, in terms of the network, in which the individuals of one of the groups expect the others will demand $\mathrm{L}$ and hence they will demand $\mathrm{H}$; and at the same time, the individuals of the other group will expect and demand the complementary decisions. In these cases, the system reaches an absorbing state, which is efficient but not equitable in the payoffs obtained by each agent. Considering the interaction network, these inequitable absorbing states can only happen if the network is bipartite, i.e. the network can be divided into two independent subnetworks such that the agents of one of them are only linked to agents of the other, and vice versa. In general, these states are rather improbable due to this topological necessity. For instance (see Figure 2), whenever the interaction network has triplets of agents or any odd cycles, the coordination in the strategies $(\mathrm{H}, \mathrm{L})$ is not stable since there is at least one pair of agents with incentives to change their current states. Note in the examples that the expected evolution is a series of continuous changes in agents' strategies between $\mathrm{H}$ and $\mathrm{L}$. This unstable pattern (which is directly related with a fractious regime that will be defined below) can persist for very long, until the only absorbing state (i.e. the EQ state) is reached.

\footnotetext{
${ }^{4}$ In networks with more than one component, there can be more types of absorbing states. In these cases, because each component is independent of the others, the absorbing state of the system is defined as the combination of the absorbing states reached by each component.
} 
A1

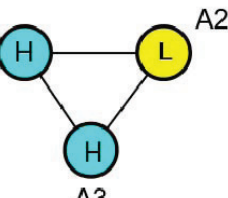
A2
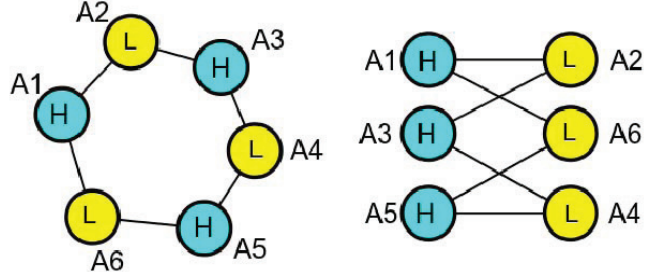

Figure 2. An inequitable state in a triplet (left graph), and in an even cycle of 6 agents (middle graph) with its corresponding bipartite representation (right graph). Agents who demand high $(\mathrm{H})$ are depicted in light blue, while those ones who demand low (L) are in light yellow. Note that for inequitable states to be absorbing, the interaction network has to be bipartite (second and third figures), i.e. it should not have odd cycles.

\subsection{The Perturbed Model}

When errors are possible in the agents' decisions ${ }^{5}$, i.e. the perturbed model, the system becomes ergodic, regardless of the interaction network. In this case, there is a unique limiting distribution -and consequently independent of the initial conditions- over the state space which determines the probability of finding the system in each of its states in the long run. This limiting distribution can be estimated by sampling just one simulation run for a sufficiently long time, and computing the fraction of the time that the system spends in each state, i.e. the occupancy distribution [39]. For a finite population and global interaction this limiting distribution concentrates on the EQ state, which is the only stochastically stable state [22].

The asymptotic behaviour is not very useful if we want to apply the model to real situations in which the "long run" is a very vague concept. For that reason, it is interesting to pay attention to the transient dynamics too, following the guidelines proposed by Axtell et al [23]. It can be shown by computer simulation that starting from random initial conditions the system quickly settles in one of two relevant regimes where it spends a considerable fraction of the time ${ }^{6}$. One of these persistent regimes, which we call EQ regime, is characterized by the EQ state and its surroundings in the state space. In the other, called fractious (FR) regime', the agents alternate their demands between $\mathrm{H}$ and L. Axtell et al [23] demonstrated that the transition time from this fractious regime to the stochastically stable state can be enormously long -the system presents broken ergodicity [42]-; in fact this time grows exponentially with the number of agents and their memory length.

\footnotetext{
${ }^{5}$ Errors refer to the noisy response explained in the model's section.

${ }^{6}$ It is also possible that the system reaches a persistent inequitable regime, where a set of agents persistently demands $\mathrm{H}$ against another set of agents that consequently demands L. Notwithstanding the topological conditions to reach this regime are very unlikely as explained in the analysis of the unperturbed model.

${ }^{7}$ We use the concept proposed originally by Axtell et al [23], although there are other names in the literature, such as "fluctuating agents" $[40,41]$, used to refer the same concept, i.e. agents that intermittently change their strategy.
} 


\section{The Equity Norm}

All the states and persistent regimes defined in the previous section correspond to a set of Markovian states of the system. However, in order to complete the analysis and discussion of the model, we also need to characterize some of the individual states in which an agent can be from the point of view of the agent's behaviour, i.e. which of the three possible decisions $\{L, M, H\}$ an agent will take in the next interaction. We say that an agent follows the equity norm whenever she demands $M$ in the next interaction (ignoring the effect of error on decisions). Obviously, this type of behaviour is directly related with the corresponding persistent regimes where the system can settle in the transient period: when all agents follow the equity norm the system reaches the EQ regime (which is equivalent to say that the population follows the equity norm).

\subsection{Clustering Effect on the Equity Norm}

It is well-known that many real social networks show a significant propensity to form groups or clusters of agents more densely interconnected among them than what could be expected by pure randomness $[26,30,43]$. A typical statistic of this property is the clustering coefficient of a network $C[30]$, measured (Eq. 1) as the average of the clustering coefficients $C_{i}$ of the agents, i.e. the proportion of links between her neighbours (triplets) divided by the number of links that could exist between all of them, which depend directly on the number of connections (degree $\mathrm{k}_{\mathrm{i}}$ ) of the agents.

$$
C=\frac{1}{N} \sum_{i}^{N} C_{i}=\frac{1}{N} \sum_{i}^{N} \frac{2}{k_{i}\left(k_{i}-1\right)} \text { \#triplets }_{i}
$$

Eq. 1

This measure of network transitivity estimates the probability that two neighbours of any agent have a link between them too, and consequently that they all form a triplet. We have seen that the existence of triplets hinders the stability of the inequitable regime, and, in the case of a state close to them it is very probable that the system falls in the trap of the fractious regime. Besides, it is also interesting to understand the effect of clustering on the equity norm. A simple analysis of the two idealized cases showed in Figure 3 should give us some insights about how clustering influences on the stability of this state (in terms of persistence in a finite time period).

The first case represents a triplet of agents initially following the equity norm, when one of them changes (mutates) her demand from $\mathrm{M}$ to $\mathrm{H}$. The topology of agents' interactions weakens the state of the mutant, who has incentives to change her strategy back because all her expected opponents demand $\mathrm{M}$. On the contrary, the other two agents do not have incentives to change their current strategy $M$ because it is successful in half of their expected interactions. In the second case, there is the same triplet but without the link between the two agents who demand $M$. Now, these last agents have incentives to change their current strategy $M$, making the equity norm less robust against random mutations. 

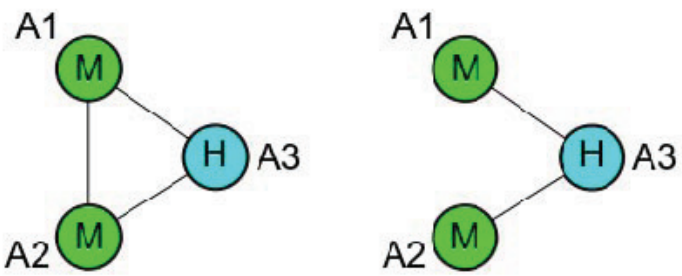

Figure 3. The effect of a mutation within a triplet (left graph), and on a non-clustered triad (right graph), when agents are initially coordinated in the equity norm and the mutant changes her demand from $\mathbf{M}$ (light green) to $\mathbf{H}$ (light blue).

Obviously, the analysis is not so trivial if the network is bigger and much more complex, but the intuition, inferred from these simple examples, is that the equity norm is much more robust against random mutations when agents are clustered than when they are not. Consequently, we should expect that the evolution of the bargaining (under the hypothesis of the model proposed in this work) tends to reach the EQ regime more frequently in networks with higher clustering. The design of experiments and the computer simulations described in the next section aim to confirm this intuition.

\subsection{The Diffusion Process of the Equity Norm}

The purpose of this section is to describe how the equity norm emerges and spreads across the population in finite time (transient dynamics). In simple and abstract terms, the dynamic process evolves as follows: the population starts from a randomly initialized state; these random initial conditions make it likely that, initially, one or more agents adopt the equity norm and coordinate with each other in small groups that reinforce the norm; if this coordination process occurs quickly, and some of these equity nuclei are able to reach a critical size (which depends on the particular properties of the network they are embedded in), then they will be able to expand their limits and grow, making the equity norm spread across the whole population. Unlike other diffusion phenomena already studied in the literature $[38,44-$ 46], the diffusion process in our model is more difficult to follow, since the adoption mechanism of the norm depends on a learning decision rule and a stochastic response. To overcome this obstacle, we propose an abstraction that captures the essence of the process and allows us to understand the effect of the network structure on the system dynamics more clearly.

In order to do so, we initially define a new unit of analysis called equity nucleus, i.e. a connected component of the subgraph of agents that follow the equity norm. At any time, there could be none, one or more equity nuclei; and we will measure their sizes and their clustering coefficients with the purpose of correlating these properties with the posterior evolution of the nuclei: they may grow until they finally invade the population or they may decrease until their disappearance in the transient period.

Second, we need to determine a metric to measure the change in an equity nucleus after a complete interaction at each time period t, i.e. every agent plays the Nash demand game with 
one of her neighbours randomly selected. To that end, we define two new concepts: the inner border and the outer border of a nucleus. Given any equity nucleus in the population (see Figure 4), its inner border is the set of agents in the nucleus who have one or more neighbours out of it, i.e. neighbours who will not play $M$ in the next interaction; its outer border is the set of agents not belonging to the nucleus who have one or more neighbours within it.

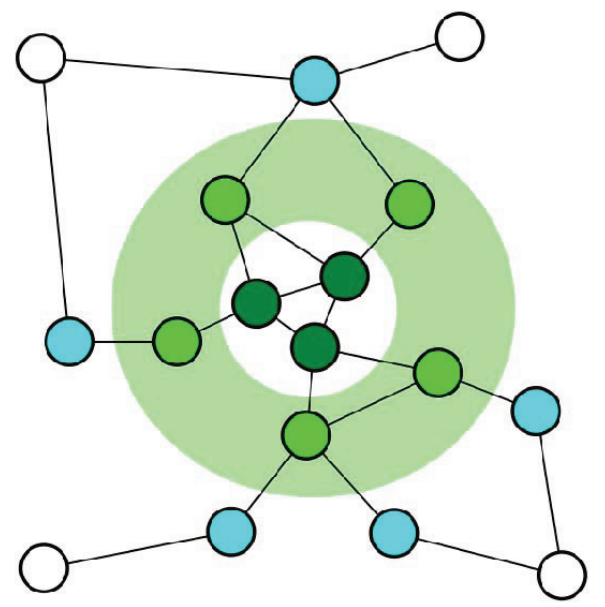

Figure 4. An equity nucleus (dark and light green agents). The inner border is made up of the (light green) agents belonging to the nucleus who have one or more neighbours out of it. The outer border consists of the (light blue) agents not belonging to the nucleus who have one or more neighbours within it.

Note that any change in a nucleus must involve one of these two borders. A nucleus can grow by adding new members of the outer border who adopt the equity norm. Similarly, a nucleus can decrease as a consequence of losing members of the inner border who leave the norm. Obviously, the real nuclei dynamics might be a little different, since in each time period there are $\mathrm{N}$ individual interactions that can modify these borders in different ways. For example, one interaction could make a border grow and the next interaction could make it decrease. Despite that, this approach is accurate enough for understanding the effect of the network structure.

Finally, we set a procedure to compute all these properties over a simulation run. Before a complete interaction at time period $t$, we identify all equity nuclei $j \in\{1, \ldots, K\}$ and their borders, and compute and collect their sizes and clustering $\left\{\mathrm{S}_{\mathrm{j}}(\mathrm{t}), \mathrm{C}_{\mathrm{j}}(\mathrm{t})\right\}_{\mathrm{j}=1}^{\mathrm{K}}$. After all agents have played the game, we compute the changes in the inner and outer borders of every nucleus. We will use the set of pairs, $\left\{S_{j}(t), C_{j}(t)\right\}^{K}{ }_{j=1}$ and $\left\{S_{j}(t+1), C_{j}(t+1)\right\}_{j=1}^{K}$, to infer some conclusions of the expected development of an equity nucleus depending on its size and clustering. 


\section{Results}

\subsection{Design of Experiments}

In the ABM model proposed, agents are embedded in a small-world interaction network (SWN from now). We have chosen the small-world algorithm by Watts and Strogatz [30] to model the interaction network because it provides a useful framework to study the clustering effect, besides other properties of the network, using only one parameter. Then, the rewiring probability is going to be the main control parameter, which will govern the network creation and its properties. The design of experiments aims to show how this kind of network family affects the system dynamics.

The parameterization of all scenarios studied in this paper corresponds to a model of $\mathrm{N}=100$ agents randomly distributed in a particular instance of the SWN for a fixed rewiring probability, and an average degree equal to 8 (for beta $=0$ and degree $=8$, the properties of the resulting network are close to the ones of the regular square lattice used in previous research [25]). Each agent is endowed with a memory of length $m=10$, randomly initialized at the beginning of a simulation run. At a time period $t$, each agent (selected in a random order) randomly selects one of her neighbours to interact. Both agents decide the best reply against the most frequent demand in their memory. However, with a small probability $\varepsilon=0.01$ an agent decides randomly between the three possible demands $\{L, M, H\}$. We have sampled 100 simulation runs during $T_{f}=2000$ time periods for each combination of parameters. This time is enough for the system to reach a persistent regime (either EQ or FR).

During a simulation run, we say that an agent follows the equity norm strongly whenever she has at least $(1-\varepsilon) \times m$ instances of $M$ in her memory. Similarly, the system reaches the FR regime whenever every agent has at least a combination of $(1-\varepsilon) \times m$ instances of both $L$ and $H^{8}$. Finally, in order to identify an equity nucleus, and its inner and outer borders, we apply the equity norm definition, and consider that an agent belongs to an equity nucleus if the mode of her memory is $M$, which is enough to guarantee that the agent will demand $M$ in the next interaction in absence of errors.

\subsection{Frequencies of Transient Regimes}

As explained before, in the transient dynamics of the system, simulations often reach one of two expected regimes: the EQ regime or the FR regime. The first one corresponds to the emergence of the equity norm, while the second represents a confusing and disordered state in agents' decisions that prevents any coordination in the bargaining. Now, the first question that arouses our interest is to understand how small-world networks condition the emergence of these regimes. To determine this influence we have computed the frequencies of both regimes when the rewiring probability $\beta$ of the network varies. Figure 5 presents the frequency of the EQ regime reached by a set of simulations at the end of the runs ${ }^{9}$.

\footnotetext{
${ }^{8}$ Note that the memory vector has a finite number of instances, so we approximate $(1-\varepsilon) \times m$ to the lower integer and $\varepsilon \times m$ to the higher integer.

${ }^{9}$ Note that since the system reaches one of the two transient regimes, the rest of the cases correspond to simulations which ended at the FR regime.
} 


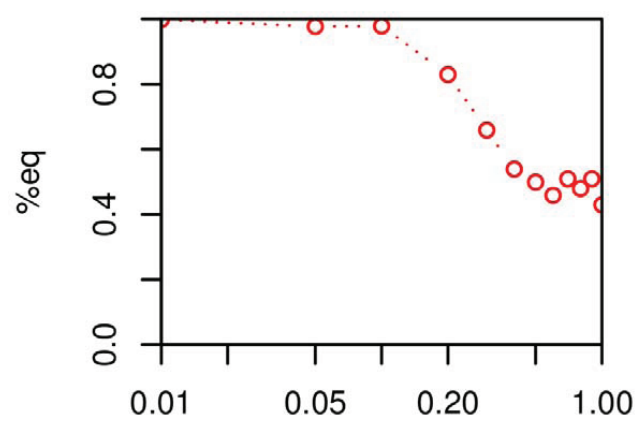

$\beta$

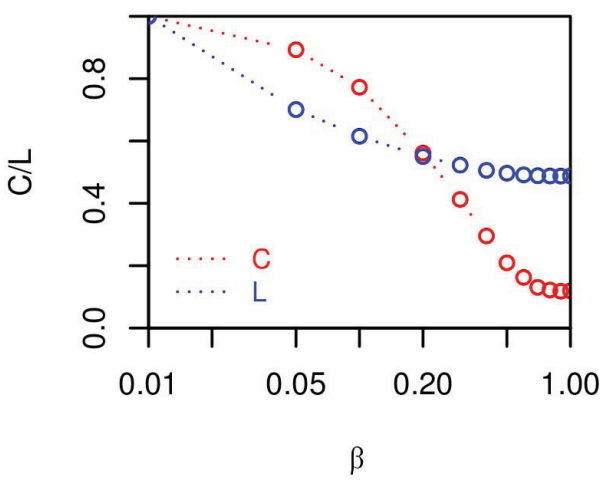

Figure 5. Above, the frequency of the EQ regime reached at the end of the simulations when the rewiring probability $\beta$ (represented in logarithmic scale on the abscissa axis) varies. Below, the average of the clustering coefficient $C$ and the average path length $L$ of the interaction networks of the simulations (both statistics are normalized dividing each value by the corresponding ones of the regular ring lattice $(\beta=0))$.

The first inference that can be made from the results is that the emergence of the EQ regime depends significantly on the rewiring probability, and more concretely on the structure of the interaction network. In the case of regular ring lattices $(\beta=0)$ characterized by the highest values of clustering and path length, the population follows the norm in (almost) all cases. As randomness increases $(\beta>0)$ the networks show lower values of clustering and average path length, and the frequency of the norm decays with them, particularly with the clustering coefficient, being finally quite close to $50 \%$ in the extreme case of pure random networks 
$(\beta=1)$. Moreover, in the well-known small-world range $(0.05<\beta<0.1)^{10}$, characterized by high values of clustering and low values of average path length, the frequency of the norm is nearly the same as in regular ring lattices. Then, it seems that the average path length does not explain the dynamics of the norm in the bargaining model (at least for small networks), which makes sense because the distance between agents, i.e. the minimum number of links between two agents, does not seem to play any role in how agents take decisions. This contrasts with the clustering coefficient, which does reflect the characteristics of the neighbourhoods, and is consequently related with the agents' interactions and the bargaining evolution.

\subsection{Equity Norm Diffusion}

In this section we characterize the diffusion process of the equity norm. We apply our particular approach based on observing the emergence and evolution of clusters of agents playing the norm -equity nuclei-. We also try to correlate the dynamics of these nuclei with their network properties, and estimate their expected change. We will see how the rewiring probability of the small-world networks conditions significantly not only the probabilities of the emergence of successful equity nuclei, but also their growing speed over the population.

The diffusion of the equity norm is quite similar to the movement of a wave of adopters in a population embedded in a social network. By randomness, one or more small groups of linked agents start to follow the equity norm (equity nuclei), and depending on their internal structure and the structure of the network that surrounds them, they have greater or lower probabilities of growing successfully by incorporating new members which modify the properties of the nuclei. Overall, if an equity nucleus reaches a critical size with particular properties, it will invade the population, but these properties will depend highly on the parameters of the interaction network.

We have analysed the observed dynamics of the equity nuclei by means of a gradient map obtained through computer simulation data (this procedure has also been used in [47]). The statistical procedure to make this sort of graph is described as follows: we start from the matrix of change $\left\{\mathrm{S}_{j}(\mathrm{t}), \mathrm{C}_{\mathrm{j}}(\mathrm{t}), \mathrm{S}_{\mathrm{j}}(\mathrm{t}+1), \mathrm{C}_{\mathrm{j}}(\mathrm{t}+1)\right\}_{\mathrm{j}=1}^{\mathrm{K}}$ that collects the size $(\mathrm{S})$ and the clustering $(\mathrm{C})$ of all equity nuclei before and after a game round, and which have been computed following the way described in previous sections. Each row element can be interpreted as a vector of change of a nucleus in the size-clustering space. Consequently, the matrix of change of a particular parameterization of the model collects a set of vectors of change of equity nuclei that happened in the corresponding simulation runs. We divide the size-clustering space in a regular square lattice. For each cell we compute the vectorial sum of all vectors with initial points included in the cell, and represent the resulting vector as an arrow of normalized magnitude (we are only interested in the direction of the expected movement). We additionally colour each square according to the probability that a nucleus of given $\mathrm{S}$ and $\mathrm{C}$ increases in size, computed as the relative frequency of occurrence in the simulated data. The gradient maps for different rewiring probabilities are shown in Figure 6.

\footnotetext{
${ }^{10}$ The small-world range is sensible to variations in the rest of the parameters, the size of the network and the average degree of the agents.
} 


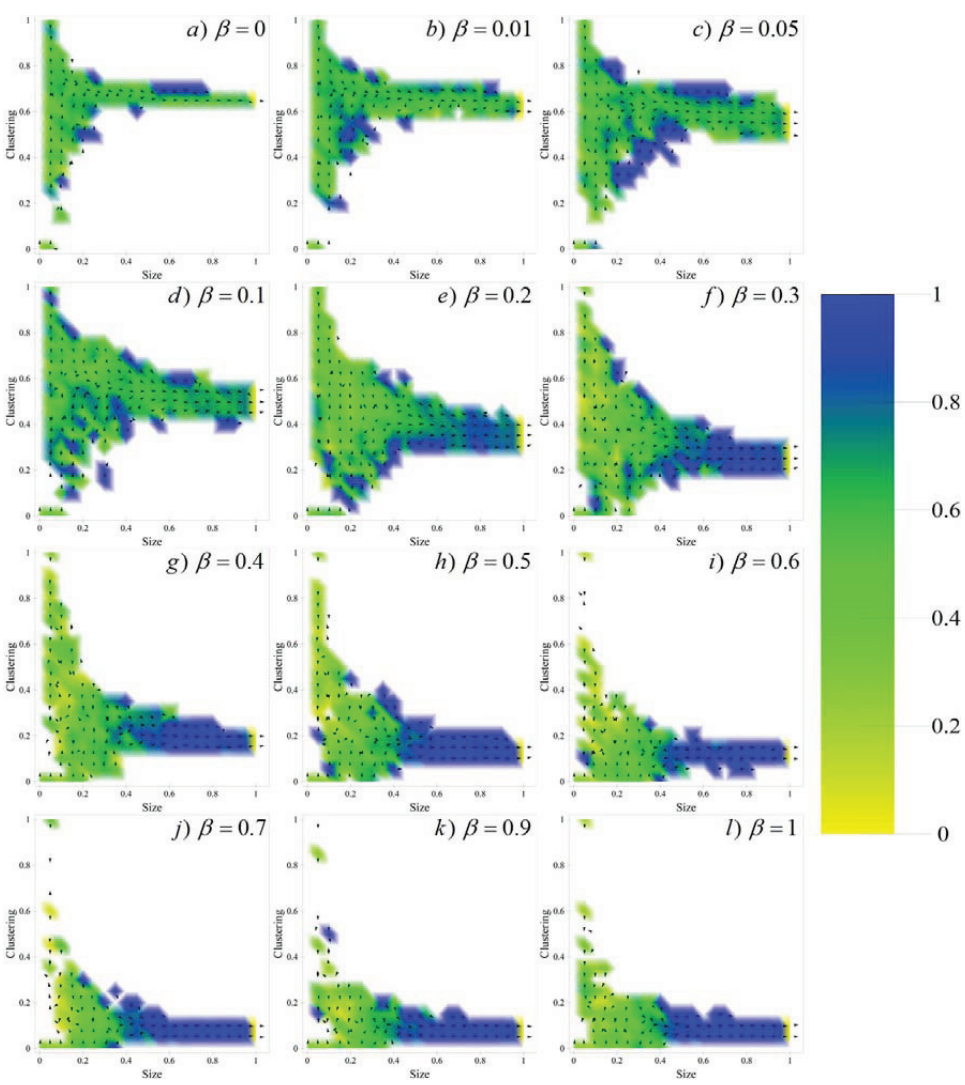

Figure 6. Gradient maps of the observed dynamics of equity nuclei for different values of the rewiring probability. Each arrow represents the direction of the change in the size-clustering space, while the colour of the cells is the probability of growing in size, which can be interpreted as a measure of the speed in nuclei growth. When there is no simulation data for a particular combination of size and clustering, the corresponding square cell of the map is coloured in white.

In most cases of Figure 6 we see two different movement regions: a first one corresponding to a developing stage, in which the equity nuclei emerge and grow slowly, and a second one corresponding to a spreading stage, in which the consolidated nuclei grow fast and invade finally the whole population.

For regular ring lattice $(\beta=0)$ and small rewiring probabilities $(\beta \leq 0.05)$, the difference between these two stages is not quite clear, and although there is a region in which nuclei emerge, grow and decay, nuclei do not need to reach a big critical size to consolidate an unstoppable 
growth. Remember that for all these cases the equity norm is always established, what is explained by the high level of clustering of these networks. Taking into account the regular structure of the interaction network it is not surprising that the spreading of the equity norm in quasi regular ring lattices is very homogeneous and slow: the norm supported by very clustered nuclei advances invading also very clustered subnetworks, and this slows down significantly the diffusion speed reflected in the gradient map by probabilities of growing close to 0.5 .

On the other hand, for greater rewiring probabilities $(\beta \geq 0.2)$ these two regions are much easier to observe in the gradient map (a green region versus a blue region). In these cases the frequency of the establishment of the equity norm decays with the rewiring probability (see Figure 5), being finally close to 0.5 in pure random networks $(\beta=1)$. The developing stage in which small nuclei grow and decay is represented by a more extensive area (green region), and the critical size necessary to start an unstoppable growth is bigger. Unlike regular ring lattices, once a consolidated nucleus emerges and the equity norm spreads over the whole population (blue region), the diffusion speed is much faster because the norm has to invade less clustered subnetworks.

We can summarize these inferences into the next statements: locally structured networks -in the sense of having more clustering- promote the emergence of the equity norm, while less locally structured networks facilitate the appearance of disordered or fractious states (according to the data of Figure 5); nevertheless, the clustering of the network can slow down the diffusion of the equity norm making more difficult the process of adoption (according to the data of Figure 6). For example, in the case of quasi regular ring lattices, an equity nucleus that invades the whole population always emerges, sooner or later, although the clustering of the network slows down the convergence to the norm. On the contrary, in more random networks, the probability of this event decreases with lower clustering values, although if an equity nucleus succeeds, the speed of the convergence to the norm is much faster.

Figure 7 shows the speed of the diffusion of the equity norm, i.e. the minimum time necessary for the whole population to converge to the norm. In accordance with previous results, the times of convergence are significantly higher for quasi regular ring lattices $(\beta \leq 0.1)$ than for more random networks $(\beta \geq 0.2)$, as a consequence of the higher resistance to adopt the norm that clustered groups of non-equity agents show in the convergence process. Note that the results represented graphically in Figure 7 correspond to the times of convergence of all simulation runs that reached the EQ regime; this percentage adds up to $100 \%$ in the case of quasi regular ring lattices, but decreases with the rewiring probability from $80 \%(\beta=0.2)$ to quite less than $50 \%(\beta=1)$. There seems to be a sort of phase change in the range $[0.1<\beta<0.2]$, coincident with a significant drop of the clustering levels of the network (see Figure 5), that explains the significant dispersion in the times of convergence for $\beta=0.2$. For rewiring probabilities greater than this value, we can infer that the time of convergence to the equity norm decreases with $\beta$, reaching the lowest value in pure random networks $(\beta=1)$. 

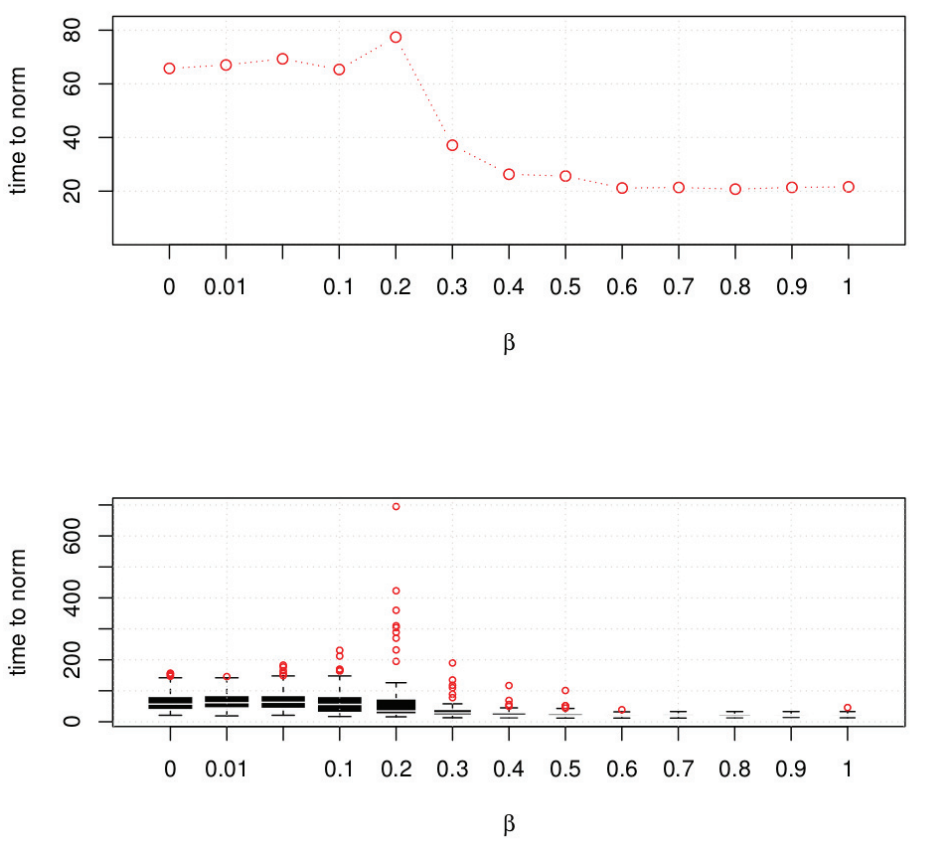

Figure 7. Above, the average of the time of convergence to the equity norm, and below the corresponding boxplot, when the rewiring probability $\beta$ varies. Note that the results are computed with the simulation runs that ended in the equity norm a number that decreases with the rewiring probability (see Figure 5 ). The range $[0.1<\beta<0.2]$ separates two system behaviours: a first one characterized by high clustering networks in which the system always reaches the EQ regime in the simulation time, and a second one characterized by significantly lower values of clustering in which the system alternates between the EQ regime and the FR regime but in cases when the system reaches the EQ regime it takes it lower times of convergence.

Finally, we have extended the computing analysis of the bargaining model by running other simulations in order to check the sensitivity of the results to changes in other parameters, mainly in the size of the population. Figure 8 shows the frequency of the EQ regime for different population sizes. Overall, these results are not qualitative different from the previous ones obtained with a population of 100 agents. Nevertheless, it is interesting to observe that the growth of the size of the network seems to promote the establishment of the equity norm in even more random realizations. For example, for $\beta=0.2$ and a number of agents greater than 400 , the equity norm is reached in almost $100 \%$ of the simulation runs, in contrast to the $80 \%$ reached by populations of 100 agents. 

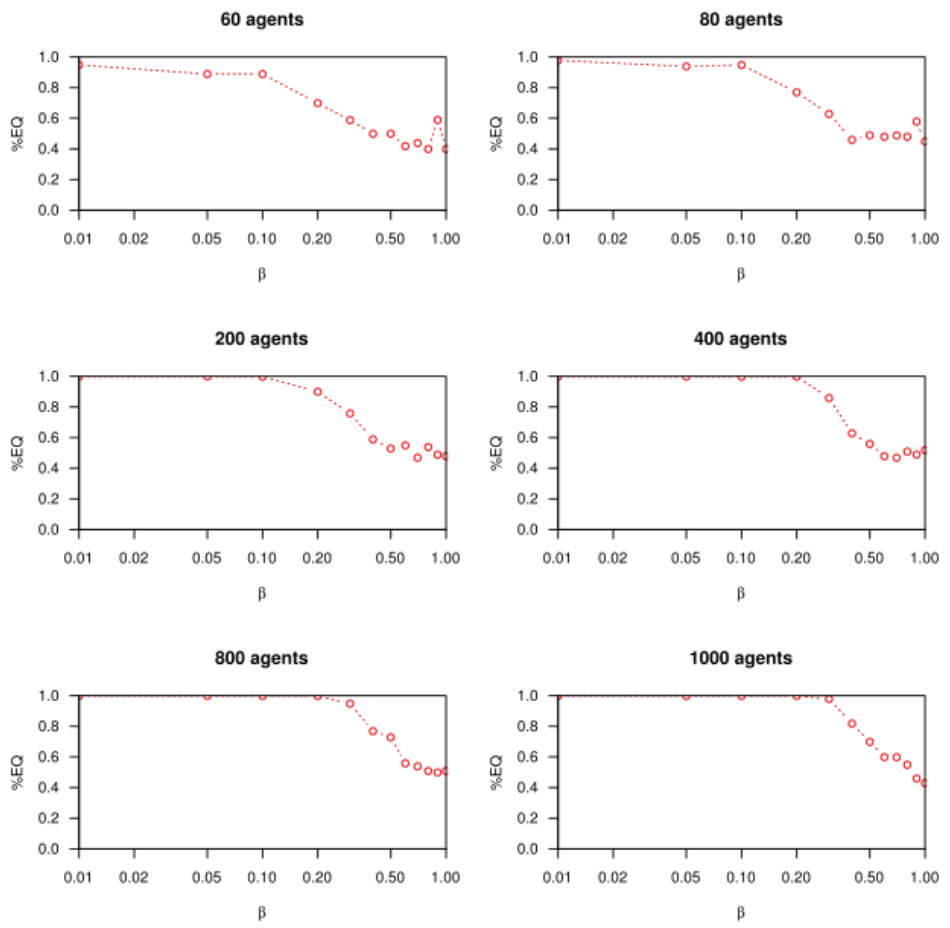

Figure 8. The frequency of the EQ regime for different sizes of the interaction network. The qualitative results do not differ from those ones analysed in the previous sections for a population of 100 agents. Regular ring lattices and networks with low rewiring probability support the dominance of the equity norm, while more random networks contribute to the emergence of fractious states.

\section{Conclusions}

In this work we have addressed the effect of topologies of interaction ranging from regular ring lattices to random networks, including small-world networks on the Nash demand game in a finite population of agents. Our analysis shows that locally structured networks -in the sense of having more clustering- promote the emergence of the equity norm, while less locally structured networks facilitate the appearance of disordered or fractious states. At the same time, results indicate that the clustering of the network can slow down the diffusion of the equity norm making more difficult the process of adoption. For example, in the case of quasi regular ring lattices, an equity nucleus that invades the whole population always emerges, sooner or later, although the clustering of the network slows down the convergence to the norm. On the contrary, in more random networks, the probability of this event decreases with lower clustering values; although if an equity nucleus succeeds, the speed of the convergence 
to the norm is much faster. Our findings seem robust to the size of population and corroborate the influence of some properties of the interaction structure in learning and evolutionary games.

\section{Acknowledgements}

We would like to thank Dr. Luis R. Izquierdo for some advice and comments on this manuscript. The authors acknowledge support from the Spanish MICINN projects CSD201000034 (SimulPast CONSOLIDER-INGENIO 2010), TIN2008-06464-C03-02 and DPI2010-16920, and by the Junta de Castilla y León, references BU034A08 and GREX251-2009.

\section{Reference List}

[1] D. Helbing and A. Johansson, "Cooperation, Norms, and Revolutions: A Unified GameTheoretical Approach," PLoS ONE, vol. 5, no. 10, Article ID e12530, 2010. doi: 10.1371/journal.pone.0012530.

[2] J. S. Coleman, Foundations of social theory, Belknap Press of Harvard University Press, Cambridge, Massachusetts, 1990.

[3] H. P. Young. "Social Norms," in S. N. Durlauf and L. E. Blume (Eds.), New Palgrave Dictionary of Economics, Macmillan, London, 2008.

[4] D. Villatoro, S. Sen and J. Sabater-Mir, "Of Social Norms and Sanctioning: A Game Theoretical Overview," International Journal of Agent Technologies and Systems, vol. 2, no. 1, pp. 1-15, 2010. doi: 10.4018/jats.2010120101.

[5] M. Hechter and K. D. Opp, Social norms, Russell Sage Foundation, New York, 2001.

[6] R. M. Axelrod, "An Evolutionary Approach to Norms," American Political Science Review, vol. 80, no. 4, pp. 1095-1111, 1986. doi: 10.2307/1960858.

[7] C. Horne, The rewards of punishment. A relational theory of norm enforcement, Stanford University Press, Stanford, CA, 2009.

[8] M. Kandori, "Social Norms and Community Enforcement," Review of Economic Studies, vol. 59, no. 1, pp. 63-80, 1992. doi: 10.2307/2297925.

[9] L. R. Izquierdo, S. S. Izquierdo and F. Vega-Redondo. "Learning and Evolutionary Game Theory," in N. M. Seel (Ed.), Encyclopedia of the Sciences of Learning, Springer, New York, 2012.

[10] D. Fudenberg and D. K. Levine, The Theory of Learning in Games, MIT Press, Cambridge, Mass, 1998.

[11] H. P. Young, Strategic Learning and Its Limits, Oxford University Press, Oxford, UK, 2004. 
[12] F. Vega-Redondo, Economics and the theory of games, Cambridge University Press, Cambridge, UK, 2003.

[13] N. Gilbert, Agent based models, Sage, London, 2007.

[14] J. M. Galán, L. R. Izquierdo, S. S. Izquierdo, J. I. Santos, R. del Olmo, A. López-Paredes and B. Edmonds, "Errors and artefacts in agent-based modelling," Journal of Artificial Societies and Social Simulation, vol. 12, no. 1, Article ID 1, 2009.

[15] R. Fuentes-Fernández, J. M. Galán, S. Hassan and F. A. Villafáñez, "Metamodelling for Agent-Based Modelling: An Application for Posted Pricing Institutions," Studies in Informatics and Control, vol. 20, no. 1, pp. 55-66, 2011.

[16] L. Tesfatsion, "Agent-based computational economics: Growing economies from the bottom up," Artificial Life, vol. 8, no. 1, pp. 55-82, 2002. doi: $10.1162 / 106454602753694765$.

[17] J. D. Farmer and D. Foley, "The economy needs agent-based modelling," Nature, vol. 460, no. 7256 , pp. 685-686, 2009. doi: 10.1038/460685a.

[18] R. Axtell, "What economic agents do: How cognition and interaction lead to emergence and complexity," The Review of Austrian Economics, vol. 20, no. 2, pp. 105122, 2007. doi: 10.1007/s11138-007-0021-5.

[19] E. Bonabeau, "Agent-based modeling: Methods and techniques for simulating human systems," Proceedings of the National Academy of Sciences of the United States of America, vol. 99, no. 2, pp. 7280-7287, 2002. doi: 10.1073/pnas.082080899.

[20] S. Hassan, M. Salgado and J. Pavón, "Friendship Dynamics: Modelling Social Relationships through a Fuzzy Agent-Based Simulation," Discrete Dynamics in Nature and Society, Article ID 765640, 2011. doi: 10.1155/2011/765640.

[21] J. F. Nash, "The Bargaining Problem," Econometrica, vol. 18, no. 2, pp. 155-162, 1950.

[22] H. P. Young, "An Evolutionary Model of Bargaining," Journal of Economic Theory, vol. 59, no. 1, pp. 145-168, 1993. doi: 10.1006/jeth.1993.1009.

[23] R. L. Axtell, J. M. Epstein and H. P. Young. "The Emergence of Classes in a Multi-Agent Bargaining Model," in S. N. Durlauf and H. P. Young (Eds.), Social Dynamics, pp. 191211, MIT Press, Cambridge, Mass./Washington, D.C., 2001.

[24] D. J. Poza, F. A. Villafáñez, J. Pajares, A. López-Paredes and C. Hernández, "New Insights on the Emergence of Classes Model," Discrete Dynamics in Nature and Society, Article ID 915279, 2011. doi: 10.1155/2011/915279.

[25] D. J. Poza, J. I. Santos, J. M. Galán and A. López-Paredes, "Mesoscopic Effects in an Agent-Based Bargaining Model in Regular Lattices," PLOS ONE, vol. 6, no. 3, Article ID e17661, 2011. doi: 10.1371/journal.pone.0017661.

[26] M. E. J. Newman, "The structure and function of complex networks," SIAM Review, vol. 45, no. 2, pp. 167-256, 2003. doi: 10.1234/12345678. 
[27] S. Schnettler, "A structured overview of 50 years of small-world research," Social Networks, vol. 31, no. 3, pp. 165-178, 2009. doi: doi: 10.1016/j.socnet.2008.12.004.

[28] I. D. S. Pool and M. Kochen, "Contacts and influence," Social Networks, vol. 1, no. 1, pp. 5-51, 1978.

[29] S. Milgram, "The small world problem," Psychology Today, vol. 1, no. 1, pp. 61-67, 1967.

[30] D. J. Watts and S. H. Strogatz, "Collective dynamics of 'small-world' networks," Nature, vol. 393, no. 6684, pp. 440-442, 1998. doi: 10.1038/30918.

[31] D. J. Watts, "Networks, Dynamics, and the Small-World Phenomenon," American Journal of Sociology, vol. 105, no. 2, pp. 493-527, 1999. doi: 10.1086/210318.

[32] E. Ahmed and A. S. Elgazzar, "On coordination and continuous hawk-dove games on small-world networks," European Physical Journal B, vol. 18, no. 1, pp. 159-162, 2000. doi: $10.1007 / \mathrm{s} 100510070088$.

[33] L. H. Shang, X. Li and X. F. Wang, "Cooperative dynamics of snowdrift game on spatial distance-dependent small-world networks," European Physical Journal B, vol. 54, no. 3, pp. 369-373, 2006. doi: 10.1140/epjb/e2006-00454-8.

[34] G. Abramson and M. Kuperman, "Social games in a social network," Physical Review E Statistical Physics, Plasmas, Fluids, and Related Interdisciplinary Topics, vol. 6303, no. 3, Article ID 030901, 2001. doi: 10.1103/PhysRevE.63.030901.

[35] X. Thibert-Plante and L. Parrott, "Prisoner's dilemma and clusters on small-world networks," Complexity, vol. 12, no. 6, pp. 22-36, 2007. doi: 10.1002/cplx.20182.

[36] M. Kirley, "Evolutionary minority games with small-world interactions," Physica A: Statistical Mechanics and its Applications, vol. 365, no. 2, pp. 521-528, 2006. doi: 10.1016/j.physa.2005.10.002.

[37] B. Xianyu, "Social preference, incomplete information, and the evolution of ultimatum game in the small world networks: An agent-based approach," Journal of Artificial Societies and Social Simulation, vol. 13, no. 2, Article ID 7, 2010.

[38] S. Delre, W. Jager and M. Janssen, "Diffusion dynamics in small-world networks with heterogeneous consumers," Computational \& Mathematical Organization Theory, vol. 13, no. 2, pp. 185-202, 2007. doi: 10.1007/s10588-006-9007-2.

[39] L. R. Izquierdo, S. S. Izquierdo, J. M. Galán and J. I. Santos, "Techniques to understand computer simulations: Markov chain analysis," Journal of Artificial Societies and Social Simulation, vol. 12, no. 1, Article ID 6, 2009.

[40] J. Gómez-Gardeñes, M. Campillo, L. M. Floría and Y. Moreno, "Dynamical organization of cooperation in complex topologies," Phys Rev Lett, vol. 98, no. 10, 2007. doi: 10.1103/PhysRevLett.98.108103.

[41] L. M. Floría, C. Gracia-Lázaro, J. Gómez-Gardeñes and Y. Moreno, "Social network reciprocity as a phase transition in evolutionary cooperation," Phys Rev E Stat Nonlinear Soft Matter Phys, vol. 79, no. 2, 2009. doi: 10.1103/PhysRevE.79.026106. 
[42] R. G. Palmer, "Broken ergodicity," Advances in Physics, vol. 31, no. 6, pp. 669-735, 1982. doi: $10.1080 / 00018738200101438$.

[43] S. Fortunato, "Community detection in graphs," Physics Reports, vol. 486, no. 3-5, pp. 75-174, 2010. doi: 10.1016/j. physrep.2009.11.002.

[44] D. J. Watts, "A simple model of global cascades on random networks," Proceedings of the National Academy of Sciences of the United States of America, vol. 99, no. 9, pp. 5766-5771, 2002. doi: 10.1073/pnas.082090499.

[45] D. López-Pintado, "Diffusion in complex social networks," Games and Economic Behavior, vol. 62, no. 2, pp. 573-590, 2008. doi: 10.1016/j.geb.2007.08.001.

[46] S. Morris, "Contagion," Review of Economic Studies, vol. 67, no. 1, pp. 57-78, 2000. doi: 10.1111/1467-937X.00121.

[47] J. M. Galán, M. M. Latek and S. M. M. Rizi, "Axelrod's Metanorm Games on Networks," PLOS ONE, vol. 6, no. 5, Article ID e20474, 2011. doi: doi:10.1371/journal.pone.0020474. 

Anexo: Evaluación de las revistas en las que han sido publicados los artículos presentados en esta tesis doctoral 

Discrete Dynamics in Nature and Society 



\section{ISI Web of Knowledge ${ }^{\text {sM }}$}

\section{Journal Citation Reports ${ }^{\oplus}$}

\section{Journal: DISCRETE DYNAMICS IN NATURE AND SOCIETY}

\begin{tabular}{|c|c|c|c|c|c|c|c|c|c|}
\hline Mark & Journal Title & ISSN & Total Cites & $\begin{array}{l}\text { Impact } \\
\text { Factor }\end{array}$ & $\begin{array}{c}\text { 5- } \\
\text { Year } \\
\text { Impact } \\
\text { Factor }\end{array}$ & $\begin{array}{c}\text { Immediacy } \\
\text { Index }\end{array}$ & $\begin{array}{l}\text { Citable } \\
\text { Items }\end{array}$ & $\begin{array}{l}\text { Cited } \\
\text { Half- } \\
\text { life }\end{array}$ & $\begin{array}{l}\text { Citing } \\
\text { Half- } \\
\text { life }\end{array}$ \\
\hline$L$ & DISCRETE DYN NAT SOC & $1026-0226$ & 391 & $\underline{0.967}$ & $\underline{1.091}$ & $\underline{0.202}$ & 109 & $\underline{3.2}$ & $\underline{6.7}$ \\
\hline & Cited Journal [00 & \multicolumn{7}{|c|}{ Citing Journal [00 } & \\
\hline
\end{tabular}

Journal Information i

Full Journal Title: DISCRETE DYNAMICS IN NATURE AND

$$
\text { SOCIETY }
$$

ISO Abbrev. Title: Discrete Dyn. Nat. Soc.

JCR Abbrev. Title: DISCRETE DYN NAT SOC

$$
\text { ISSN: } 1026-0226
$$

Issues/Year: 4

Language: ENGLISH

Eigenfactor $^{\mathrm{TM}}$ Metrics
Eigenfactor ${ }^{\mathrm{TM}}$ Score
0.00160
Article Influence ${ }^{\mathrm{TM}}$
Score
0.290

Journal Country/Territory: UNITED STATES

Publisher: HINDAWI PUBLISHING CORPORATION

Publisher Address: 410 PARK AVENUE, 15TH FLOOR, \#287 PMB, NEW YORK, NY 10022

Subject Categories: MATHEMATICS, INTERDISCIPLINARY

APPLICATIONS SCOPE NOTE
VIEW JOURNAL SUMMARY LIST
MULTIDISCIPLINARY SCIENCES SCOPE NOTE
VIEW JOURNAL SUMMARY LIST
SIEW CATEGORY DATA

Journal Rank in Categories: 百 JOURNAL RANKING

\section{Journal Impact Factor i}

Cites in 2010 to items published in: $2009=68$ $2008=79$

Sum: 147

Calculation: Cites to recent items Number of recent items
Number of items published in: $2009=94$

$2008=58$

Sum: 152

5-Year Journal Impact Factor i 
Cites in $\{2010\}$ to items published in: $2009=68$

$$
2008=79
$$

$2007=112$

$2006=38$

$2005=14$

Sum: 311

Calculation: $\underline{\text { Cites to recent items }}$ Number of recent items

$\frac{311}{285}=\mathbf{1 . 0 9 1}$

Number of items published in: $2009=94$

$2008=58$

$2007=46$

$2006=63$

$2005=24$

Sum: 285

Journal Self Cites i

The tables show the contribution of the journal's self cites to its impact factor. This information is also represented in the cited journal graph.

\begin{tabular}{|l|l|l|l|l|}
\hline Total Cites & 391 & & Self Cites & $90(23 \%$ of 391$)$ \\
\hline $\begin{array}{l}\text { Cites to Years Used in } \\
\text { Impact Factor Calculation }\end{array}$ & 147 & $\begin{array}{l}\text { Self Cites to Years Used } \\
\text { in Impact Factor Calculation }\end{array}$ & $40(27 \%$ of 147$)$ \\
\hline Impact Factor & 0.967 & Impact Factor without Self Cites & 0.704 \\
\hline
\end{tabular}

Journal Immediacy Index i

Cites in 2010 to items published in $2010=22$

Number of items published in $2010=109$

Calculation: $\underline{\text { Cites to current items }} \quad \underline{22} \quad \mathbf{0 . 2 0 2}$

Number of current items $\quad \frac{22}{109}$

\section{Journal Cited Half-Life i}

The cited half-life for the journal is the median age of its items cited in the current JCR year. Half of the citations to the journal are to items published within the cited half-life.

Cited Half-Life: 3.2 years

Breakdown of the citations to the journal by the cumulative percent of 2010 cites to items published in the following years:

\begin{tabular}{|l|r|r|r|r|r|r|r|r|r|r|r|}
\hline Cited Year & $\mathbf{2 0 1 0}$ & 2009 & $\mathbf{2 0 0 8}$ & 2007 & 2006 & 2005 & 2004 & 2003 & 2002 & 2001 & $2000-$ all \\
\hline \# Cites from 2010 & 22 & 68 & 79 & 112 & 38 & 14 & 1 & 0 & 11 & 9 & 37 \\
\hline Cumulative \% & 5.63 & 23.02 & 43.22 & 71.87 & 81.59 & 85.17 & 85.42 & 85.42 & 88.24 & 90.54 & 100 \\
\hline
\end{tabular}

Cited Half-Life Calculations:

The cited half-life calculation finds the number of publication years from the current JCR year that account for $50 \%$ of citations received by the journal. Read help for more information on the calculation.

Cited Journal Graph i)

Click here for Cited Journal data table

This graph shows the distribution by cited year of citations to items published in the journal DISCRETE DYN NAT SOC.

Citations to the journal (per cited year)

- The white/grey division indicates the cited half-life (if $<10.0$ ). Half of the journal's cited items were published more recently than the cited half-life.

- The top (gold) portion of each column indicates Journal Self Citations: citations to items in the journal from items in the 


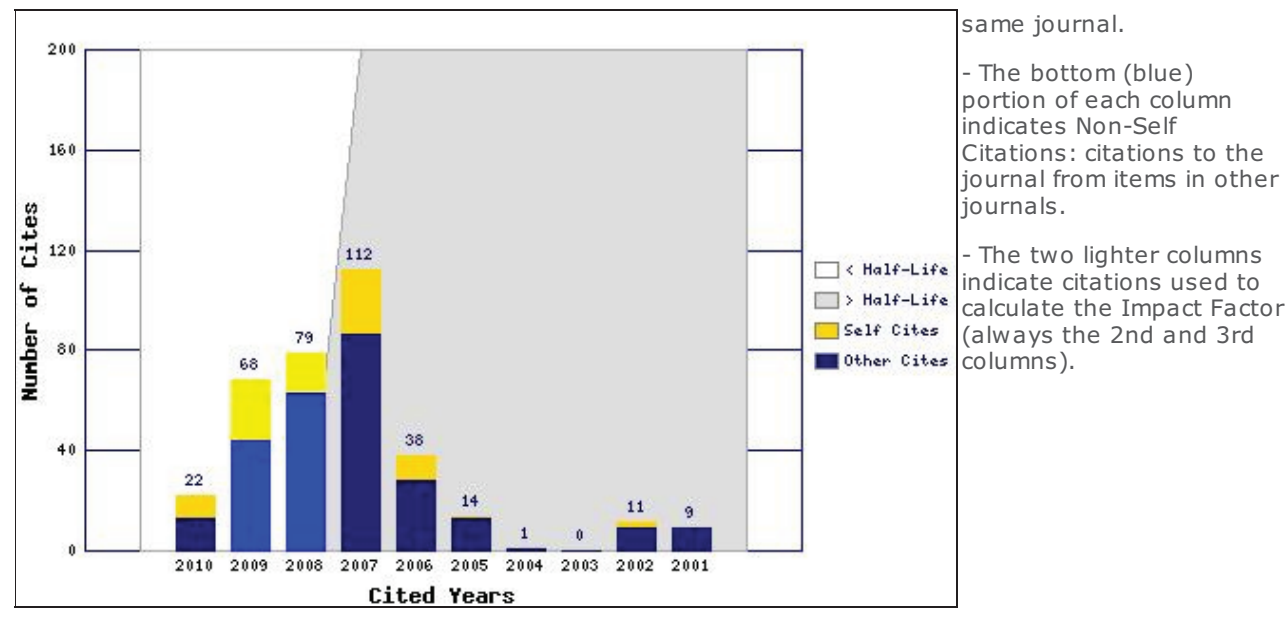

Journal Citing Half-Life i

The citing half-life for the journal is the median age of the items the journal cited in the current JCR year. Half of the citations in the journal are to items published within the citing half-life.

Citing Half-Life: 6.7 years

Breakdown of the citations from the journal by the cumulative percent of 2010 cites to items published in the following years:

\begin{tabular}{|l|r|r|r|r|r|r|r|r|r|r|r|}
\hline Cited Year & 2010 & 2009 & 2008 & 2007 & 2006 & 2005 & 2004 & 2003 & 2002 & 2001 & $2000-$ all \\
\hline \# Cites from 2010 & 113 & 283 & 282 & 214 & 199 & 141 & 164 & 123 & 125 & 88 & 962 \\
\hline Cumulative \% & 4.19 & 14.70 & 25.17 & 33.11 & 40.50 & 45.73 & 51.82 & 56.38 & 61.02 & 64.29 & 100 \\
\hline
\end{tabular}

Citing Half-Life Calculations:

The citing half-life calculation finds the number of publication years from the current JCR year that account for $50 \%$ of citations in the journal. Read help for more information on the calculation.

\section{Citing Journal Graph i}

Click here for Citing Journal data table

This graph shows the distribution by cited year of citations from current-year items in the journal DISCRETE DYN NAT SOC.

Citations from the journal (per cited year)

$$
\begin{aligned}
& \text { - The white/grey division } \\
& \text { indicates the citing half-life } \\
& \text { (if < 10.0). Half of the } \\
& \text { citations from the journal's } \\
& \text { current items are to items } \\
& \text { published more recently } \\
& \text { than the citing half-life. } \\
& \text { - The top (gold) portion of } \\
& \text { each column indicates } \\
& \text { Journal Self-Citations: } \\
& \text { citations from items in the } \\
& \text { journal to items in the } \\
& \text { same journal. } \\
& \text { - The bottom (blue) } \\
& \text { portion of each column } \\
& \text { indicates Non-Self } \\
& \text { Citations: citations from } \\
& \text { the journal to items in } \\
& \text { other journals. }
\end{aligned}
$$




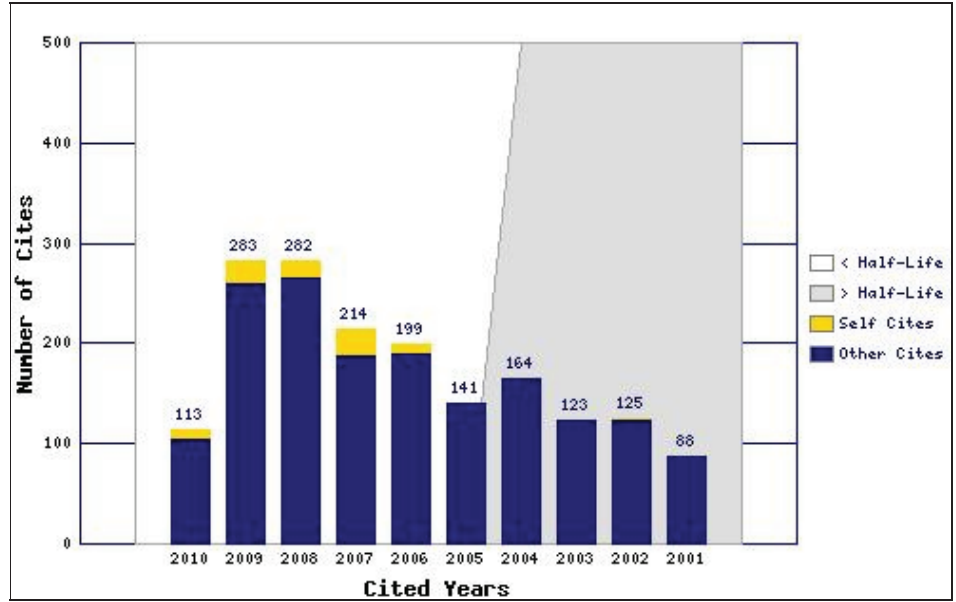

Journal Source Data i

\begin{tabular}{|l|r|r|r|r|}
\hline & \multicolumn{3}{|c|}{ Citable items } & \\
\cline { 2 - 5 } & Articles & Reviews & Combined & Other items \\
\hline Number in JCR year 2010 (A) & 108 & 1 & 109 & 0 \\
\hline Number of references (B) & 2548 & 13 & 2561 & 0.00 \\
\hline Ratio (B/A) & 23.6 & 13.0 & 23.5 & 0.0 \\
\hline
\end{tabular}

Acceptable Use Policy

Copyright (c) 2012 Thomson Reuters. 


\section{ISI Web of Knowledge}

\section{Journal Citation Reports ${ }^{\oplus}$}

10. WELCOME ? HELP $\checkmark$ Mast $\begin{aligned} & \text { MARED } \\ & \text { LOUURNAL }\end{aligned}$

2010 JCR Science Edition

Rank in Category: DISCRETE DYNAMICS IN NATURE AND SOCIETY

Journal Ranking i

For 2010, the journal DISCRETE DYNAMICS IN NATURE AND SOCIETY has an Impact Factor of 0.967 .

This table shows the ranking of this journal in its subject categories based on Impact Factor.

\begin{tabular}{|l|c|c|c|}
\hline \multicolumn{1}{|c|}{ Category Name } & $\begin{array}{c}\text { Total } \\
\text { Journals } \\
\text { in Category }\end{array}$ & $\begin{array}{c}\text { Journal } \\
\text { Rank } \\
\text { in Category }\end{array}$ & $\begin{array}{c}\text { Quartile } \\
\text { in } \\
\text { Category }\end{array}$ \\
\hline $\begin{array}{l}\text { MATHEMATICS, INTERDISCIPLINARY } \\
\text { APPLICATIONS }\end{array}$ & 93 & 46 & Q2 \\
\hline MULTIDISCIPLINARY SCIENCES & 59 & 19 & Q2 \\
\hline
\end{tabular}

Category Box Plot i

For 2010, the journal DISCRETE DYNAMICS IN NATURE AND SOCIETY has an Impact Factor of 0.967 .

This is a box plot of the subject category or categories to which the journal has been assigned. It provides information about the distribution of journals based on Impact Factor values. It shows median, 25th and 75th percentiles, and the extreme values of the distribution.

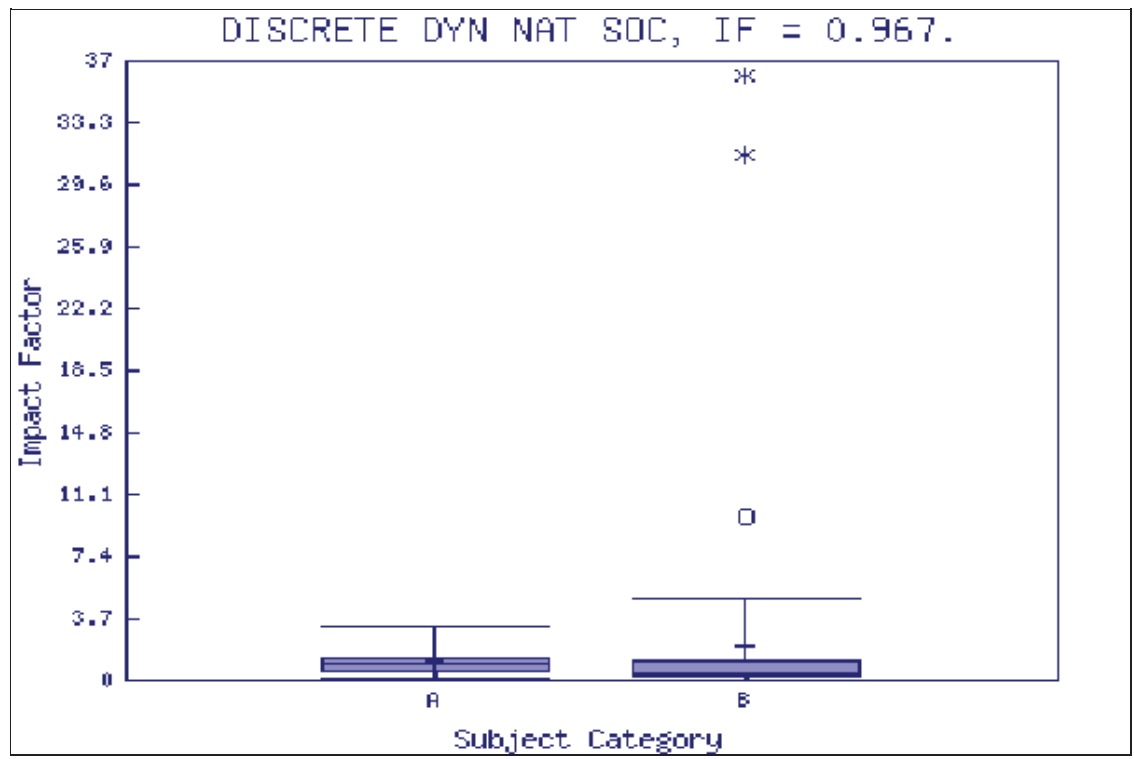


ISI Web of Knowledge ${ }^{\text {sM }}$

Journal Citation Reports ${ }^{\oplus}$

WELCOME ? HELP $\$$ MARKED RETURN TO

Impact Factor Trend Graph: DISCRETE

DYNAMICS IN NATURE AND SOCIETY

Click on the "Return to Journal" button to view the full journal information.

DISCRETE DYNAMICS IN NATURE AND SOCIETY

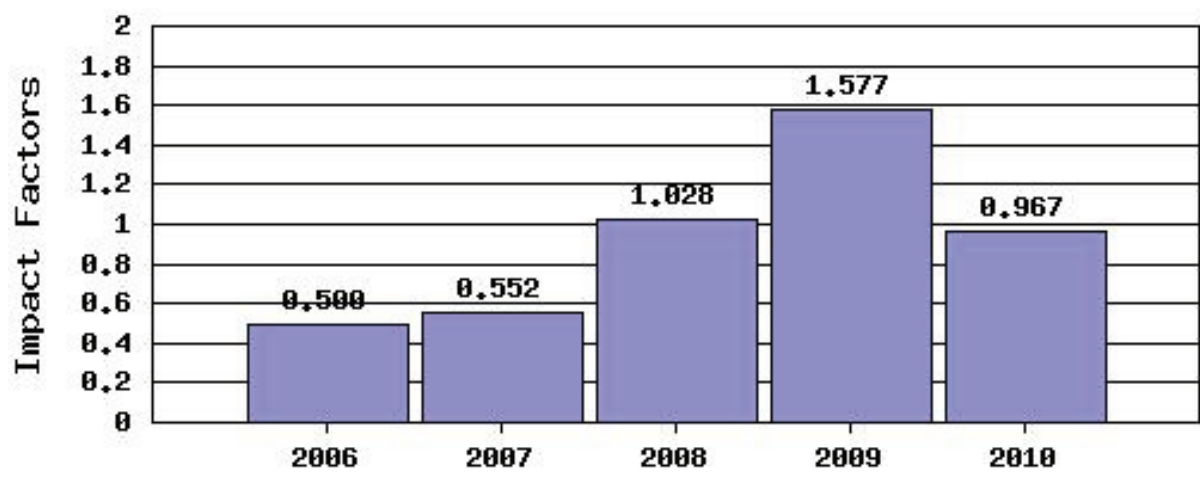

JCR Years

* Im pact Factor - - see below for calculations

The journal impact factor is a measure of the frequency with which the "average article" in a journal has been cited in a particular year. The impact factor will help you evaluate a journal's relative importance, especially when you compare it to others in the same field. For more bibliometric data and information on this and other journal titles click on the "Return to Journal" button.

NOTE: Title changes and coverage changes may result in no impact factor for one or more years in the above graph.

\section{Impact Factor}

Cites in 2010 to articles published in:

$\begin{array}{lll}2009 & 68 & \begin{array}{l}\text { Number of articles } \\ \text { published in: }\end{array} \\ 2008 & & \\ = & 79 & \\ \text { Sum: } & 147 & \end{array}$

Sum: 147

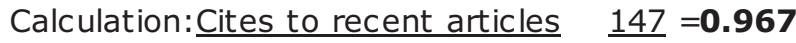

$\begin{array}{ll}2009 & 94 \\ = & \\ 2008 & 58 \\ = & \\ \text { Sum: } & 152\end{array}$

Sum: 152 


\section{Impact Factor}

Cites in 2009 to articles published in:

\begin{tabular}{|c|c|c|c|}
\hline $\begin{array}{l}2008 \\
=\end{array}$ & 83 & $\begin{array}{l}\text { Number of articles } \\
\text { published in: }\end{array}$ & $\begin{array}{l}2008 \\
=\end{array}$ \\
\hline $\begin{array}{l}2007 \\
=\end{array}$ & 81 & & $\begin{array}{l}2007 \\
=\end{array}$ \\
\hline Sum: & 164 & & Sum: \\
\hline
\end{tabular}

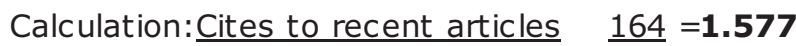

Number of recent articles 104

\begin{tabular}{|c|c|c|c|c|c|}
\hline \multicolumn{6}{|l|}{2008 Impact Factor } \\
\hline $\begin{array}{l}\text { Cites in } 2008 \text { to articles } \\
\text { published in: }\end{array}$ & $\begin{array}{l}2007 \\
=\end{array}$ & 56 & $\begin{array}{l}\text { Number of articles } \\
\text { published in: }\end{array}$ & $\begin{array}{l}2007 \\
=\end{array}$ & 46 \\
\hline & $\begin{array}{l}2006 \\
=\end{array}$ & 56 & & $\begin{array}{l}2006 \\
=\end{array}$ & 63 \\
\hline & Sum: & 112 & & Sum: & 109 \\
\hline \multicolumn{4}{|c|}{ Calculation: $\frac{\text { Cites to recent articles }}{\text { Number of recent articles }} \frac{112}{109}=\mathbf{1 . 0 2 8}$} & & \\
\hline \multicolumn{6}{|l|}{2007 Impact Factor } \\
\hline \multirow[t]{3}{*}{$\begin{array}{l}\text { Cites in } 2007 \text { to articles } \\
\text { published in: }\end{array}$} & $\begin{array}{l}2006 \\
=\end{array}$ & 30 & $\begin{array}{l}\text { Number of articles } \\
\text { published in: }\end{array}$ & $\begin{array}{l}2006 \\
=\end{array}$ & 63 \\
\hline & $\begin{array}{l}2005 \\
=\end{array}$ & 18 & & $\begin{array}{l}2005 \\
=\end{array}$ & 24 \\
\hline & Sum: & $: 48$ & & Sum: & 87 \\
\hline \multicolumn{6}{|c|}{ Calculation: $\frac{\text { Cites to recent articles }}{\text { Number of recent articles }} \frac{48}{87}=\mathbf{0 . 5 5 2}$} \\
\hline \multicolumn{6}{|l|}{2006 Impact Factor } \\
\hline \multirow[t]{3}{*}{$\begin{array}{c}\text { Cites in } 2006 \text { to articles } \\
\text { published in: }\end{array}$} & $\begin{array}{l}2005 \\
=\end{array}$ & 16 & \multirow[t]{3}{*}{$\begin{array}{l}\text { Number of articles } \\
\text { published in: }\end{array}$} & $\begin{array}{l}2005 \\
=\end{array}$ & 24 \\
\hline & $\begin{array}{l}2004 \\
=\end{array}$ & 7 & & $\begin{array}{l}2004 \\
=\end{array}$ & 22 \\
\hline & Sum: & $: 23$ & & Sum: & 46 \\
\hline
\end{tabular}

Calculation: $\underline{\text { Cites to recent articles }} \underline{23}=\mathbf{0 . 5 0 0}$

Number of recent articles 46 

PLoS ONE 



\section{ISI Web of Knowledge}

\section{Journal Citation Reports ${ }^{\oplus}$}

\begin{tabular}{|c|c|c|}
\hline WELCOME & ? HELP & $\begin{array}{l}7 \text { MARKED } \\
\text { LIST }\end{array}$ \\
\hline
\end{tabular}

2010 JCR Science Edition

\section{Journal: PLoS One}

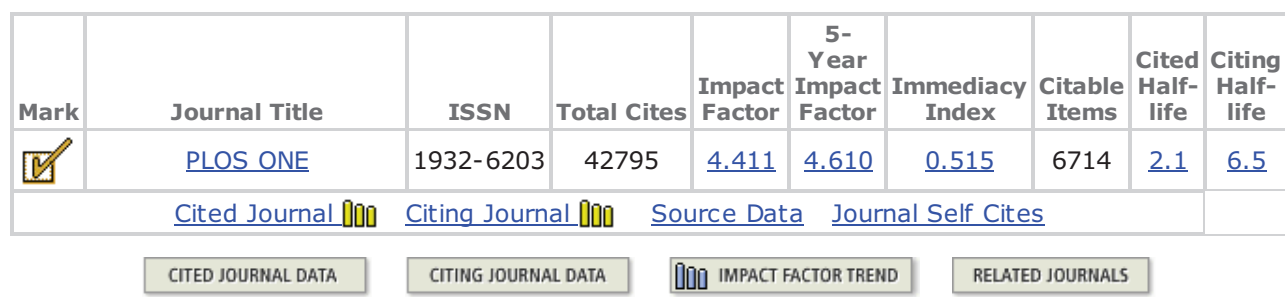

Journal Information i

Full Journal Title: PLoS One

ISO Abbrev. Title: PLoS One

JCR Abbrev. Title: PLOS ONE

Issues/Year: 0

$$
\text { ISSN: } 1932-6203
$$

Language: ENGLISH

Journal Country/Territory: UNITED STATES

Publisher: PUBLIC LIBRARY SCIENCE

Publisher Address: 185 BERRY ST, STE 1300, SAN FRANCISCO, CA 94107

Subject Categories: BIOLOGY SCOPE NOTE

8 VIEW JOURNAL SUMMARY LIST RI VIEW CATEGORY DATA

Journal Rank in Categories: 百 JOURNAL RANKING

Journal Impact Factor i

Cites in 2010 to items published in: $2009=16131$ Number of items published in: $2009=4403$

$$
\begin{array}{ll}
2008=15273 & 2008=2717 \\
\text { Sum: } 31404 & \text { Sum: } 7120
\end{array}
$$

Calculation: $\underline{\text { Cites to recent items }} \quad \frac{31404}{7120}=\mathbf{4 . 4 1 1}$

Number of recent items $\quad 7120$

\section{5-Year Journal Impact Factor i}

Cites in $\{2010\}$ to items published in: $2009=16131$ Number of items published in: $2009=4403$

$\begin{array}{ll}2008=15273 & 2008=2717 \\ 2007=6934 & 2007=1230 \\ 2006=768 & 2006=137 \\ 2005=19 & 2005=0 \\ \text { Sum: } 39125 & \text { Sum: } 8487\end{array}$


Journal Self Cites i

The tables show the contribution of the journal's self cites to its impact factor. This information is also represented in the cited journal graph.

\begin{tabular}{|l|l|l|l|}
\hline Total Cites & 42795 & & 3097 (7\% of 42795) \\
\hline $\begin{array}{l}\text { Cites to Years Used in } \\
\text { Impact Factor Calculation }\end{array}$ & 31404 & $\begin{array}{l}\text { Self Cites to Years Used } \\
\text { in Impact Factor Calculation }\end{array}$ & 2174 (6\% of 31404) \\
\hline Impact Factor & 4.411 & Impact Factor without Self Cites & 4.105 \\
\hline
\end{tabular}

\section{Journal Immediacy Index i}

Cites in 2010 to items published in $2010=3460$

Number of items published in $2010=6714$

Calculation: Cites to current items $\quad \underline{3460}=\mathbf{0 . 5 1 5}$

Number of current items $\quad \frac{3460}{6714}$

\section{Journal Cited Half-Life i}

The cited half-life for the journal is the median age of its items cited in the current JCR year. Half of the citations to the journal are to items published within the cited half-life.

Cited Half-Life: 2.1 years

Breakdown of the citations to the journal by the cumulative percent of 2010 cites to items published in the following years:

\begin{tabular}{|l|r|l|l|r|r|r|r|r|r|r|r|}
\hline Cited Year & 2010 & 2009 & 2008 & 2007 & 2006 & 2005 & 2004 & 2003 & 2002 & 2001 & $2000-$ all \\
\hline \# Cites from 2010 & 3460 & 16131 & 15273 & 6934 & 768 & 19 & 7 & 9 & 12 & 4 & 178 \\
\hline Cumulative \% & 8.09 & 45.78 & 81.47 & 97.67 & 99.46 & 99.51 & 99.53 & 99.55 & 99.57 & 99.58 & 100 \\
\hline
\end{tabular}

Cited Half-Life Calculations:

The cited half-life calculation finds the number of publication years from the current JCR year that account for $50 \%$ of citations received by the journal. Read help for more information on the calculation.

\section{Cited Journal Graph i}

Click here for Cited Journal data table

This graph shows the distribution by cited year of citations to items published in the journal PLOS ONE. Citations to the journal (per cited year)

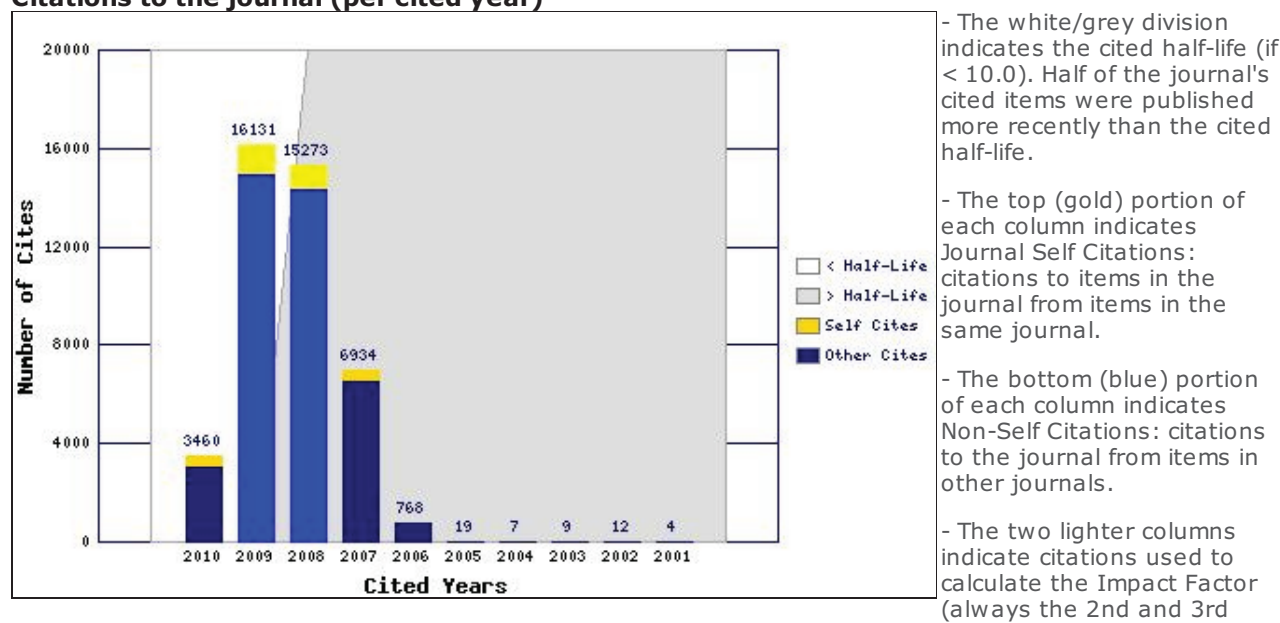


columns).

Journal Citing Half-Life i

The citing half-life for the journal is the median age of the items the journal cited in the current JCR year. Half of the citations in the journal are to items published within the citing half-life.

Citing Half-Life: 6.5 years

Breakdown of the citations from the journal by the cumulative percent of 2010 cites to items published in the following years:

\begin{tabular}{|c|c|c|c|c|c|c|c|c|c|c|c|}
\hline Cited Year & 2010 & 2009 & 2008 & 2007 & 2006 & 2005 & 2004 & 2003 & 2002 & 2001 & 2000-a \\
\hline \# Cites from 2010 & 8390 & 29631 & 32336 & 30131 & 27080 & 24491 & 22007 & 19187 & 17023 & 14978 & 10021 \\
\hline Cumulative \% & 2.58 & 11.68 & 21.62 & 30.87 & 39.20 & 46.72 & 53.48 & 59.38 & 64.61 & 69.21 & 10 \\
\hline
\end{tabular}

Citing Half-Life Calculations:

The citing half-life calculation finds the number of publication years from the current JCR year that account for $50 \%$ of citations in the journal. Read help for more information on the calculation.

Citing Journal Graph $\mathbf{i}$

Click here for Citing Journal data table

This graph shows the distribution by cited year of citations from current-year items in the journal PLOS ONE. Citations from the journal (per cited year)

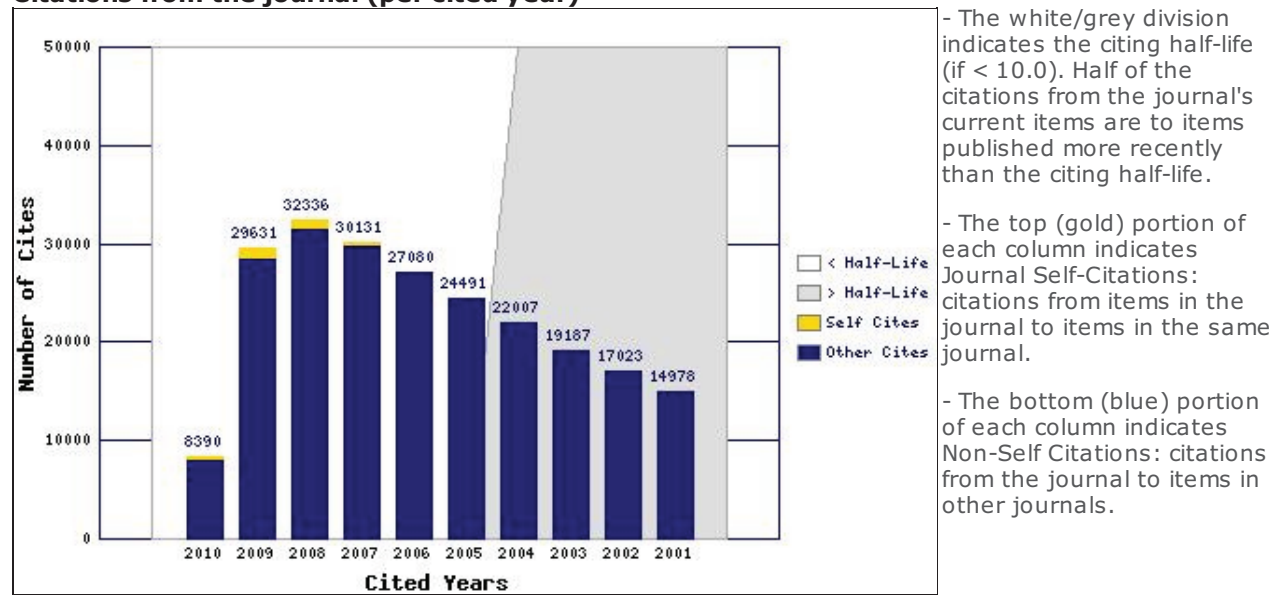

Journal Source Data i

\begin{tabular}{|l|r|r|r|r|}
\hline & \multicolumn{3}{|c|}{ Citable items } & \\
\cline { 2 - 5 } & Articles & Reviews & Combined & Other items \\
\hline Number in JCR year 2010 (A) & 6682 & 32 & 6714 & 0 \\
\hline Number of references (B) & 315634 & 3693 & 319327 & 0.00 \\
\hline Ratio (B/A) & 47.2 & 115.4 & 47.6 & 0.0 \\
\hline
\end{tabular}




\section{ISI Web of Knowledge}

\section{Journal Citation Reports}

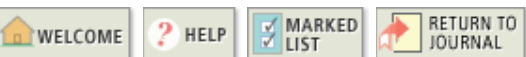

2010 JCR Science Edition

\section{Rank in Category: PLoS One}

\section{Journal Ranking i}

For 2010, the journal PLoS One has an Impact Factor of 4.411.

This table shows the ranking of this journal in its subject categories based on Impact Factor.

\begin{tabular}{|l|c|c|c|}
\hline Category Name & $\begin{array}{c}\text { Total Journals } \\
\text { in Category }\end{array}$ & $\begin{array}{c}\text { Journal Rank } \\
\text { in Category }\end{array}$ & $\begin{array}{c}\text { Q uartile } \\
\text { in Category }\end{array}$ \\
\hline BIOLOGY & 86 & 12 & Q1 \\
\hline
\end{tabular}

Category Box Plot i

For 2010, the journal PLos One has an Impact Factor of 4.411.

This is a box plot of the subject category or categories to which the journal has been assigned. It provides information about the distribution of journals based on Impact Factor values. It shows median, 25th and 75th percentiles, and the extreme values of the distribution.

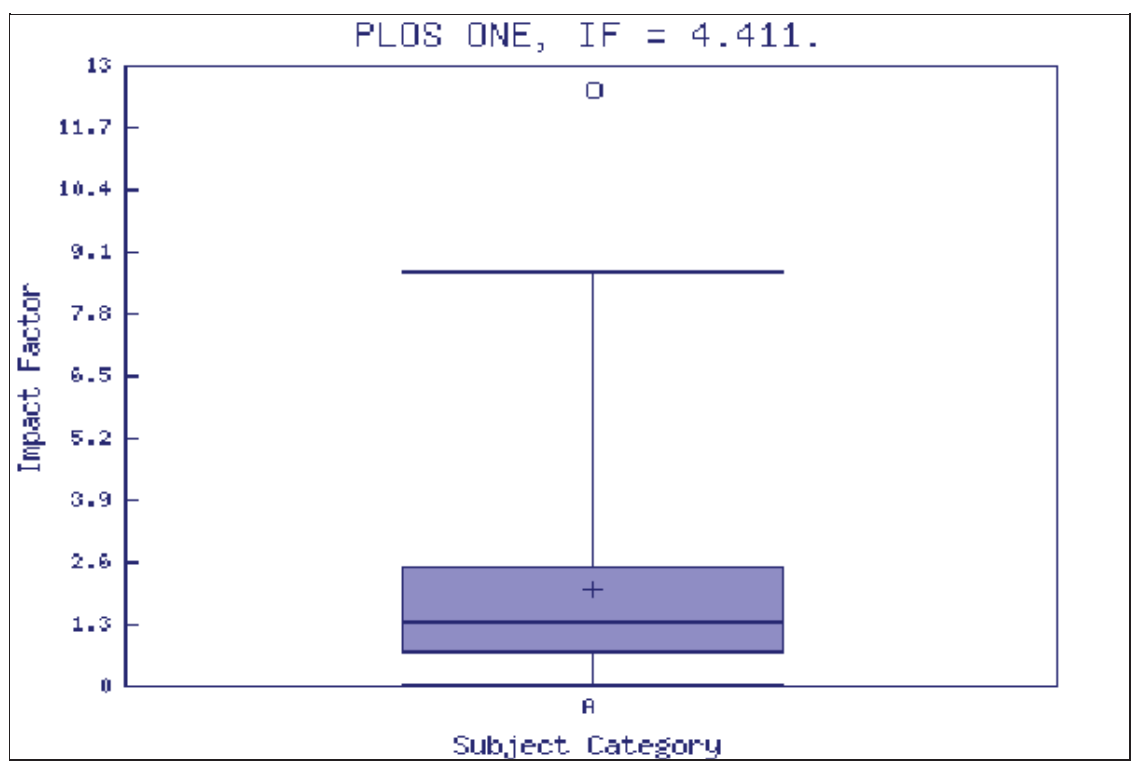


ISI Web of Knowledge ${ }^{\text {sM }}$

\section{Journal Citation Reports ${ }^{\oplus}$}

WELCOME ? HELP $\approx$ MARKED RETURN TO

Impact Factor Trend Graph: PLos One

Click on the "Return to Journal" button to view the full journal information.

PLos One

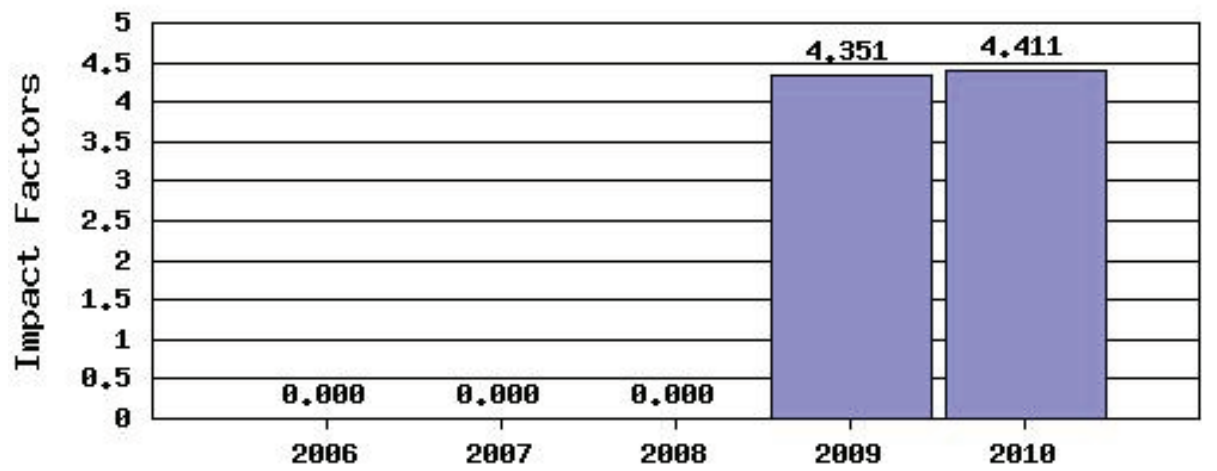

JCR Years

* Im pact Factor -- see below for calculations

The journal impact factor is a measure of the frequency with which the "average article" in a journal has been cited in a particular year. The impact factor will help you evaluate a journal's relative importance, especially when you compare it to others in the same field. For more bibliometric data and information on this and other journal titles click on the "Return to Journal" button.

NOTE: Title changes and coverage changes may result in no impact factor for one or more years in the above graph.

\section{Impact Factor}

Cites in 2010 to articles published in:

$\begin{array}{lll}2009 & 16131 & \begin{array}{l}\text { Number of articles } \\ \text { published in: }\end{array} \\ = & & \\ = & 15273 & \end{array}$

Sum: 31404
20094403

$=$

2008

2717

Sum: 7120

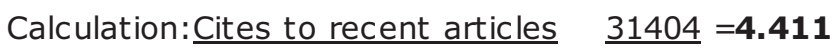

Number of recent articles 7120 


\section{Impact Factor}

Cites in 2009 to articles 200810624 Number of articles 20082725 published in:

$\begin{array}{lll}2008 & 10624 & \begin{array}{l}\text { Number of articles } \\ \text { published in: }\end{array} \\ 2007 & 6580 & \end{array}$$$
=
$$

Sum: 17204

Calculation: Cites to recent articles $\quad \underline{17204}=\mathbf{4 . 3 5 1}$

Number of recent articles 3954
20071229

Sum: 3954 